\title{
Analytical Data Report of Grab Samples Collected From 200-ZP-1 Remedial Action Wells
}

Michael Lindberg

February 2010

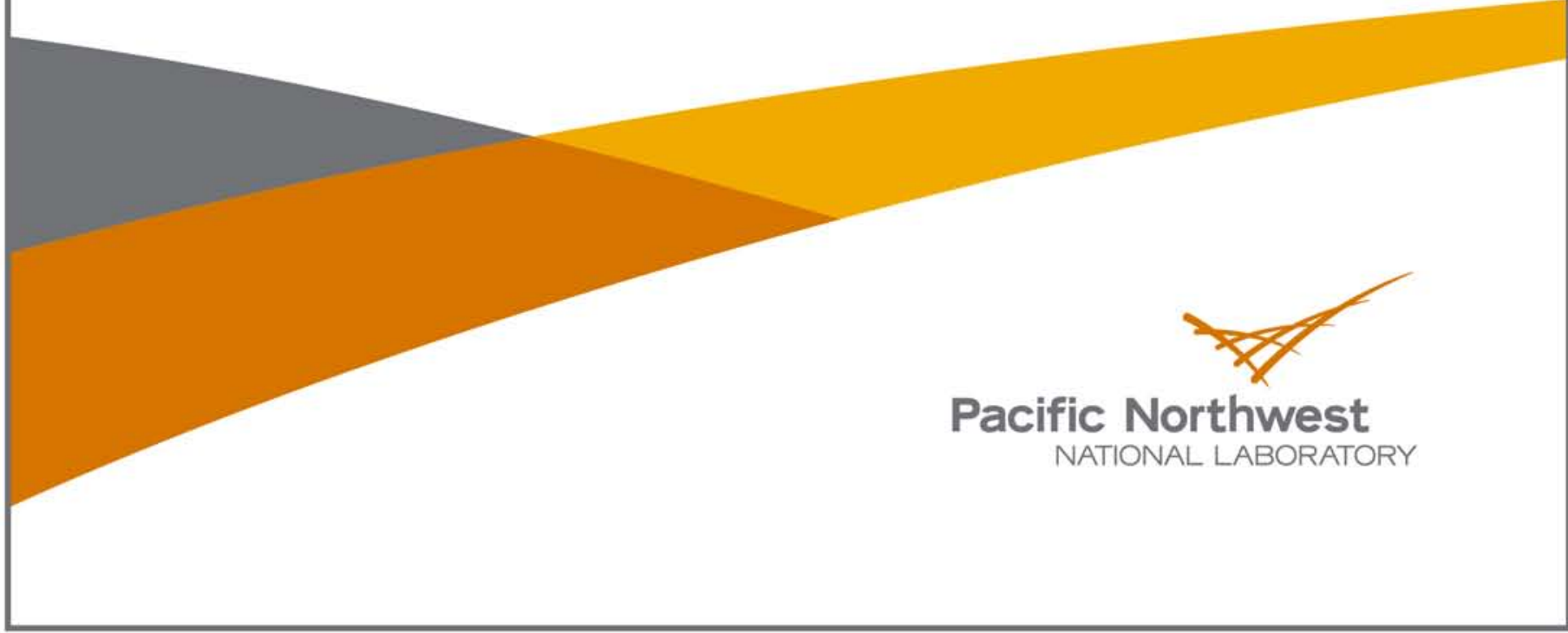




\title{
DISCLAIMER
}

This report was prepared as an account of work sponsored by an agency of the United States Government. Neither the United States Government nor any agency thereof, nor Battelle Memorial Institute, nor any of their employees, makes any warranty, express or implied, or assumes any legal liability or responsibility for the accuracy, completeness, or usefulness of any information, apparatus, product, or process disclosed, or represents that its use would not infringe privately owned rights. Reference herein to any specific commercial product, process, or service by trade name, trademark, manufacturer, or otherwise does not necessarily constitute or imply its endorsement, recommendation, or favoring by the United States Government or any agency thereof, or Battelle Memorial Institute. The views and opinions of authors expressed herein do not necessarily state or reflect those of the United States Government or any agency thereof.

\author{
PACIFIC NORTHWEST NATIONAL LABORATORY \\ operated by \\ BATTELLE \\ for the \\ UNITED STATES DEPARTMENT OF ENERGY \\ under Contract DE-AC05-76RL01830
}

Printed in the United States of America
Available to DOE and DOE contractors from the Office of Scientific and Technical Information,
P.O. Box 62, Oak Ridge, TN 37831-0062;
ph: (865) 576-8401
fax: $(865)$ 576-5728
email: reports@adonis.osti.gov

\begin{abstract}
Available to the public from the National Technical Information Service, U.S. Department of Commerce, 5285 Port Royal Rd., Springfield, VA 22161 ph: (800) 553-6847 fax: $(703) 605-6900$ email: orders@ntis.fedworld.gov online ordering: http://www.ntis.gov/ordering.htm
\end{abstract}

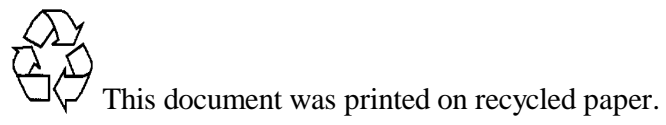




\title{
Analytical Data Report of Grab Samples Collected From 200-ZP-1 Remedial Action Wells
}

\author{
M Lindberg
}

February 2010

Prepared for the U.S. Department of Energy under Contract DE-AC05-76RL01830 
$02 / 19 / 1013: 30$

To: Dale Dyekman

From: Michael J. Lindberg

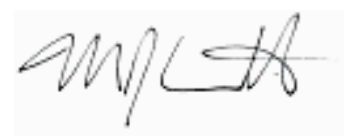

Environmental Sciences Laboratory

Energy and Environment Directorate, Pacific Northwest National Laboratory

Subject: Analytical Data Report for Sediment Samples Collected From 200-ZP-1 Remedial Action Wells, Sample Delivery Group ESL090002, SAF Number F09-012

This letter contains the following information for sample delivery group ESL090002

- Cover Sheet

- Narrative

- Analytical Results

- Quality Control

- Chain of Custodies 


\section{Introduction}

Between February 12, 2009 and August 3, 2009 sediment samples were received from 200-ZP-1 Remedial Action Wells for geochemical studies.

\section{Analytical Results/Methodology}

The analyses for this project were performed at the 325 building located in the 300 Area of the Hanford Site. The analyses were performed according to Pacific Northwest National Laboratory (PNNL) approved procedures and/or nationally recognized test procedures. The data sets include the sample identification numbers, analytical results, estimated quantification limits (EQL), and quality control data.

\section{Quality Control}

The preparatory and analytical quality control requirements, calibration requirements, acceptance criteria, and failure actions are defined in the on-line QA plan "Conducting Analytical Work in Support of Regulatory Programs" (CAW). This QA plan implements the Hanford Analytical Services Quality Assurance Requirements Documents (HASQARD) for PNNL.

\section{Definitions}

Dup Duplicate

RPD Relative Percent Difference

NR No Recovery (percent recovery less than zero)

ND Non-Detectable

\%REC Percent Recovery

\section{Sample Receipt}

Samples were received with a chain of custody (COC) and were analyzed according to the sample identification numbers supplied by the client. All Samples were refrigerated upon receipt until prepared for analysis.

All samples were received with custody seals intact unless noted in the Case Narrative.

\section{Holding Times}

Holding time is defined as the time from sample preparation to the time of analyses. The prescribed holding times were met for all analytes unless noted in the Case Narrative.

\section{Analytical Results}

All reported analytical results meet the requirements of the CAW or client specified SOW unless noted in the case narrative. 


\section{Case Narrative Report}

\section{Hold Time:}

Sample 0902002-01 failed hold criteria for Total Carbon-NP.

Sampled $=02 / 09 / 09$ 12:57

Prepared $=03 / 10 / 09$ 14:04

Analyzed $=03 / 10 / 09$ 15:40

Sampled- $>$ Analyzed $>28.00$ days

Sample 0902002-01 failed hold criteria for Total Inorganic Carbon-NP.

Sampled $=02 / 09 / 0912: 57$

Prepared $=03 / 11 / 09$ 08:19

Analyzed $=03 / 11 / 09$ 10:22

Sampled- $>$ Analyzed $>28.00$ days

Sample 0902002-05 failed hold criteria for Total Carbon-NP.

Sampled $=$ 03/03/09 13:07

Prepared $=04 / 15 / 09$ 09:55

Analyzed $=04 / 16 / 09$ 11:04

Sampled- $>$ Analyzed $>28.00$ days

Sample 0902002-05 failed hold criteria for Total Inorganic Carbon-NP.

Sampled $=03 / 03 / 09$ 13:07

Prepared $=04 / 16 / 09$ 14:58

Analyzed $=04 / 21 / 0914: 43$

Sampled- $>$ Analyzed $>28.00$ days

Sample 0902002-26 failed hold criteria for Total Carbon-NP.

Sampled $=04 / 22 / 09$ 08:55

Prepared $=06 / 02 / 09$ 08:25

Analyzed $=06 / 02 / 09$ 10:37

Sampled- $>$ Analyzed $>28.00$ days

Sample 0902002-26 failed hold criteria for Total Inorganic Carbon-NP.

Sampled $=04 / 22 / 09$ 08:55

Prepared $=06 / 02 / 09$ 08:49

Analyzed $=06 / 02 / 09$ 13:40

Sampled- $>$ Analyzed $>28.00$ days

Sample 0902002-27 failed hold criteria for Total Carbon-NP.

Sampled $=04 / 27 / 09$ 10:46

Prepared $=06 / 02 / 09$ 08:25

Analyzed $=06 / 02 / 09$ 10:48

Sampled->Analyzed $>28.00$ days

Sample 0902002-27 failed hold criteria for Total Inorganic Carbon-NP.

Sampled $=04 / 27 / 09$ 10:46

Prepared $=06 / 02 / 09$ 08:49

Analyzed = 06/02/09 13:50

Sampled- $>$ Analyzed $>28.00$ days 


\section{Case Narrative Report}

\section{Hold Time:}

Sample 0902002-28 failed hold criteria for Total Carbon-NP.

Sampled $=04 / 29 / 09$ 08:15

Prepared $=06 / 02 / 09$ 08:25

Analyzed $=06 / 02 / 09$ 10:58

Sampled- $>$ Analyzed $>28.00$ days

Sample 0902002-28 failed hold criteria for Total Inorganic Carbon-NP.

Sampled $=04 / 29 / 09$ 08:15

Prepared $=06 / 02 / 09$ 08:49

Analyzed =06/02/09 14:02

Sampled- $>$ Analyzed $>28.00$ days

Sample 0902002-29 failed hold criteria for Total Carbon-NP.

Sampled $=05 / 01 / 09$ 11:40

Prepared $=06 / 02 / 09$ 08:25

Analyzed $=06 / 02 / 09$ 11:09

Sampled- $>$ Analyzed $>28.00$ days

Sample 0902002-29 failed hold criteria for Total Inorganic Carbon-NP.

Sampled $=05 / 01 / 09$ 11:40

Prepared $=06 / 02 / 09$ 08:49

Analyzed $=06 / 02 / 09$ 14:12

Sampled- $>$ Analyzed $>28.00$ days

Sample 0902002-31 failed hold criteria for Total Carbon-NP.

Sampled $=05 / 05 / 0908: 25$

Prepared $=06 / 02 / 09$ 08:25

Analyzed $=06 / 02 / 09$ 11:33

Sampled- $>$ Analyzed $>28.00$ days

Sample 0902002-31 failed hold criteria for Total Inorganic Carbon-NP.

Sampled $=05 / 05 / 09$ 08:25

Prepared $=06 / 02 / 09$ 08:49

Analyzed $=06 / 02 / 09$ 14:30

Sampled- $>$ Analyzed $>28.00$ days

Sample 0902002-37 failed hold criteria for Total Carbon-NP.

Sampled $=06 / 26 / 09$ 08:45

Prepared $=10 / 05 / 09$ 10:19

Analyzed $=10 / 05 / 09$ 11:34

Sampled- $>$ Analyzed $>28.00$ days

Sample 0902002-37 failed hold criteria for Total Inorganic Carbon-NP.

Sampled $=06 / 26 / 09$ 08:45

Prepared $=10 / 05 / 09$ 10:19

Analyzed $=10 / 05 / 09$ 14:03

Sampled- $>$ Analyzed $>28.00$ days 


\section{Case Narrative Report}

\section{Preparation Blank (PB):}

QC Sample 9E07004-BLK1 failed criteria for Antimony 121 in ICPMS-RCRA-WE.

$\mathrm{MDL}=0.000658 \mathrm{ug} / \mathrm{g}$

$\mathrm{MRL}=0.000658 \mathrm{ug} / \mathrm{g}$

Result $=0.0140 \mathrm{ug} / \mathrm{g}$

Criterion $=1 \times$ MRL

Sample results associated with this batch were less than the EQL. There should be no impact to the data as reported.

\section{Duplicate (DUP):}

Duplicate RPD for Chloride (47.7\%) was above the acceptance limit (35) in 9C10004-DUP1 for Anions by IC-WE

All other duplicates and QC associated with the batch were in limits. Duplicate failure may be due to sample heterogeneity. There should be no impact to sample data as reported.

Duplicate RPD for Antimony 121 (44.5\%) was above the acceptance limit (35) in 9E07002-DUP1 for ICPMS-RCRA-WE The sample and duplicate values were less than 10 times the EQL. The 35\% criterion does not apply.

\section{Laboratory Control Samples (LCS):}

Bismuth, Lithium, Phosphorus, Strontium, and Zirconium are not present in the LCS. All analytes present in the LCS were in limits. There should be no impact to data as reported.

\section{Post Spike (PS):}

Post-Spike Recovery for Lead 208 (65.4\%) was outside acceptance limits (75-125) in 9E07006-PS1 for ICPMS-RCRA-AE The native sample concentration was greater than 5 times the spike concentration. There should be not impact to data as reported.

Post-Spike Recovery for Aluminum (407\%) was outside acceptance limits (75-125) in 9E12001-PS1 for ICP-OES Vadose-AE The native sample concentration was greater than 5 times the spike concentration. There should be not impact to data as reported.

Post-Spike Recovery for Calcium (386\%) was outside acceptance limits (75-125) in 9E12001-PS1 for ICP-OES Vadose-AE The native sample concentration was greater than 5 times the spike concentration. There should be not impact to data as reported.

Post-Spike Recovery for Iron (538\%) was outside acceptance limits (75-125) in 9E12001-PS1 for ICP-OES Vadose-AE

The native sample concentration was greater than 5 times the spike concentration. There should be not impact to data as reported.

Post-Spike Recovery for Manganese (126\%) was outside acceptance limits (75-125) in 9E12001-PS1 for ICP-OES Vadose-AE The native sample concentration was greater than 5 times the spike concentration. There should be not impact to data as reported.

Post-Spike Recovery for Sodium (127\%) was outside acceptance limits (75-125) in 9E12002-PS1 for ICP-OES Vadose-WE The native sample concentration was greater than 5 times the spike concentration. There should be not impact to data as reported.

Post-Spike Recovery for Sodium (136\%) was outside acceptance limits (75-125) in 9E12003-PS1 for ICP-OES Vadose-WE The native sample concentration was greater than 5 times the spike concentration. There should be not impact to data as reported.

Post-Spike Recovery for Sodium (132\%) was outside acceptance limits (75-125) in 9E12005-PS1 for ICP-OES Vadose-WE The native sample concentration was greater than 5 times the spike concentration. There should be not impact to data as reported.

\section{Matrix Spike (MS):}

No Discrepancies Noted.

\section{Other QC Criteria:}

No Discrepancies Noted. 


\section{DISCLAIMER}

This report was prepared as an account of work sponsored by an agency of the United States Government. Neither the United States Government nor any agency thereof, nor Battelle Memorial Institute, nor any of their employees, makes any warranty, express or implied, or assumes any legal liability or responsibility for the accuracy, completeness, or usefulness of any information, apparatus, product, or process disclosed, or represents that its use would not infringe privately owned rights. Reference herein to any specific commercial product, process, or service by trade name, trademark, manufacturer, or otherwise does not necessarily constitute or imply its endorsement, recommendation, or favoring by the United States Government or any agency thereof, or Battelle Memorial Institute. The views and opinions of authors expressed herein do not necessarily state or reflect those of the United States Government or any agency thereof. 
The following analyses were performed on the following samples included in this report:

Metals 1:1 DI Water Extract by ICPMS

Metals Acid Extract by ICPMS

1:1 DI Water Extract

AGG-TOC-001

Anions By Ion Chromatography

Carbon, Total, Combustion or Oxidation

GEA No Preparation

Inorganic Carbon, Total, Combustion or Oxidation

Metals 1:1 Water Extract by ICPOES

Metals Acid Extract by ICPOES

Moisture Content

Nitric Acid Digestion

Percent Solids 


$\begin{array}{lll}\text { HEIS No. } & \text { Laboratory ID } & \text { Matrix } \\ \text { B1YD07 } & 0902002-01 & \text { SOIL } \\ \text { B1YD08 } & 0902002-02 & \text { SOIL } \\ \text { B1YD09 } & 0902002-03 & \text { SOIL } \\ \text { B1YD74 } & 0902002-04 & \text { SOIL } \\ \text { B1YND4 } & 0902002-05 & \text { SOIL } \\ \text { B1YND5 } & 0902002-06 & \text { SOIL } \\ \text { B1YND7 } & 0902002-07 & \text { SOIL } \\ \text { B1YM90 } & 0902002-08 & \text { SOIL } \\ \text { B1YM91 } & 0902002-09 & \text { SOIL } \\ \text { B1YND6 } & 0902002-10 & \text { SOIL } \\ \text { B1YD75 } & 0902002-11 & \text { SOIL } \\ \text { B1YM92 } & 0902002-12 & \text { SOIL } \\ \text { B1YM93 } & 0902002-13 & \text { SOIL } \\ \text { B1YNV5 } & 0902002-14 & \text { SOIL } \\ \text { B1YD76 } & 0902002-15 & \text { SOIL } \\ \text { B1YD88 } & 0902002-16 & \text { SOIL } \\ \text { B1YD77 } & 0902002-17 & \text { SOIL } \\ \text { B1YD78 } & 0902002-18 & \text { SOIL } \\ \text { B1YD79 } & 0902002-19 & \text { SOIL } \\ \text { B1YND9 } & 0902002-20 & \text { SOIL } \\ \text { B1YNF0 } & 0902002-21 & \text { SOIL } \\ \text { B1YNF1 } & 0902002-22 & \text { SOIL } \\ \text { B1YD80 } & 0902002-23 & \text { SOIL } \\ \text { B1YD81 } & 0902002-24 & \text { SOIL } \\ \text { B1YD82 } & 0902002-25 & \text { SOIL } \\ \text { B1YNF2 } & 0902002-26 & \text { SOIL } \\ \text { B1YNF3 } & 0902002-27 & \text { SOIL } \\ \text { B1YNF4 } & 0902002-28 & \text { SOIL } \\ \text { B1YNF5 } & 0902002-29 & \text { SOIL } \\ \text { B1YNF6 } & 0902002-30 & \text { SOIL } \\ \text { B1YD83 } & 0902002-31 & \text { SOIL } \\ \text { B1YM97 } & 0902002-37 & \text { SOIL }\end{array}$

\begin{tabular}{|c|c|}
\hline Date Collected & $\begin{array}{l}\text { Date Received } \\
2 / 12 / 09 \quad 13: 50\end{array}$ \\
\hline 2/9/09 $\quad 12: 57$ & $2 / 12 / 09 \quad 13: 50$ \\
\hline 3/2/09 10:00 & $3 / 12 / 09 \quad 10: 30$ \\
\hline 3/3/09 09:00 & $3 / 12 / 09 \quad 10: 30$ \\
\hline 3/4/09 15:08 & $3 / 12 / 09 \quad 10: 30$ \\
\hline 3/3/09 13:07 & 4/1/09 13:00 \\
\hline 4/1/09 09:05 & 4/1/09 13:00 \\
\hline 3/27/09 09:30 & 4/1/09 13:00 \\
\hline $3 / 26 / 09 \quad 12: 56$ & 4/1/09 13:00 \\
\hline 4/6/09 08:02 & 4/9/09 $14: 15$ \\
\hline 4/3/09 $\quad 12: 00$ & 4/9/09 $14: 15$ \\
\hline $3 / 28 / 09 \quad 13: 45$ & 4/9/09 $\quad 14: 15$ \\
\hline 4/10/09 12:10 & $4 / 16 / 09 \quad 12: 00$ \\
\hline 4/15/09 08:05 & $4 / 16 / 09 \quad 12: 00$ \\
\hline 4/15/09 07:30 & $4 / 16 / 09 \quad 12: 00$ \\
\hline 4/20/09 08:48 & $4 / 27 / 09 \quad 13: 10$ \\
\hline 4/21/09 $08: 48$ & $4 / 27 / 09 \quad 13: 10$ \\
\hline 4/22/09 07:06 & $4 / 27 / 09 \quad 13: 10$ \\
\hline $4 / 22 / 09 \quad 16: 23$ & $4 / 27 / 09 \quad 13: 10$ \\
\hline 4/24/09 07:50 & $4 / 27 / 09 \quad 13: 10$ \\
\hline 4/9/09 $\quad 15: 25$ & $4 / 30 / 09 \quad 13: 00$ \\
\hline 4/9/09 $\quad 15: 25$ & $4 / 30 / 09 \quad 13: 00$ \\
\hline 4/20/09 08:25 & $4 / 30 / 09 \quad 13: 00$ \\
\hline 4/24/09 $08: 30$ & 4/30/09 13:00 \\
\hline 4/28/09 09:00 & $4 / 30 / 09 \quad 13: 00$ \\
\hline 4/29/09 08:54 & $4 / 30 / 09 \quad 13: 00$ \\
\hline 4/22/09 $08: 55$ & $5 / 13 / 09 \quad 13: 00$ \\
\hline 4/27/09 10:46 & $5 / 13 / 09 \quad 13: 00$ \\
\hline 4/29/09 08:15 & $5 / 13 / 09 \quad 13: 00$ \\
\hline 5/1/09 11:40 & $5 / 13 / 09 \quad 13: 00$ \\
\hline 5/6/09 $\quad 15: 22$ & $5 / 13 / 09 \quad 13: 00$ \\
\hline 5/5/09 $08: 25$ & $5 / 13 / 09 \quad 13: 00$ \\
\hline 6/26/09 08:45 & 8/3/09 13:00 \\
\hline
\end{tabular}




\section{Wet Chemistry}

Moisture Content (\% by Weight) by AGG-WC-001

\begin{tabular}{|c|c|c|c|c|c|}
\hline Lab ID & HEIS No. & Results & EQL & Analyzed & Batch \\
\hline 0902002-01 & B1YD07 & $3.94 \mathrm{E} 0$ & $\mathrm{~N} / \mathrm{A}$ & $3 / 16 / 09$ & 9C09001 \\
\hline 0902002-02 & B1YD08 & $4.66 \mathrm{E} 0$ & N/A & $3 / 26 / 09$ & $9 \mathrm{C} 23002$ \\
\hline 0902002-03 & B1YD09 & $5.30 \mathrm{E} 0$ & N/A & $3 / 26 / 09$ & 9C23002 \\
\hline 0902002-04 & B1YD74 & $9.40 \mathrm{E} 0$ & N/A & $3 / 26 / 09$ & 9C23002 \\
\hline 0902002-05 & B1YND4 & $6.57 \mathrm{E}-1$ & N/A & $4 / 08 / 09$ & 9D06001 \\
\hline 0902002-06 & B1YND5 & $6.23 \mathrm{E} 0$ & N/A & $4 / 08 / 09$ & 9D06001 \\
\hline 0902002-07 & B1YND7 & 4.46E0 & $\mathrm{N} / \mathrm{A}$ & $4 / 08 / 09$ & 9D06001 \\
\hline 0902002-08 & B1YM90 & $1.65 \mathrm{E} 1$ & N/A & $4 / 08 / 09$ & 9D06001 \\
\hline 0902002-09 & B1YM91 & $1.00 \mathrm{E} 1$ & N/A & $4 / 16 / 09$ & 9D06001 \\
\hline 0902002-10 & B1YND6 & $1.44 \mathrm{E} 1$ & N/A & $4 / 22 / 09$ & 9D06001 \\
\hline 0902002-11 & B1YD75 & $9.98 \mathrm{E} 0$ & N/A & $4 / 22 / 09$ & 9D06001 \\
\hline 0902002-12 & B1YM92 & $6.59 \mathrm{E} 0$ & N/A & $4 / 22 / 09$ & 9D06001 \\
\hline 0902002-13 & B1YM93 & $1.56 \mathrm{E} 1$ & N/A & $4 / 22 / 09$ & 9D06001 \\
\hline 0902002-14 & B1YNV5 & $8.52 \mathrm{E} 0$ & N/A & $4 / 22 / 09$ & 9D06001 \\
\hline 0902002-15 & B1YD76 & $1.25 \mathrm{E} 1$ & N/A & $6 / 22 / 09$ & 9E29002 \\
\hline 0902002-16 & B1YD88 & $1.31 \mathrm{E} 1$ & N/A & $6 / 22 / 09$ & 9E29002 \\
\hline 0902002-17 & B1YD77 & $1.77 \mathrm{E} 1$ & N/A & $6 / 22 / 09$ & 9E29002 \\
\hline 0902002-18 & B1YD78 & $2.74 \mathrm{E} 1$ & N/A & $6 / 22 / 09$ & 9E29002 \\
\hline 0902002-19 & B1YD79 & 2.99E1 & N/A & $6 / 22 / 09$ & 9E29002 \\
\hline 0902002-20 & B1YND9 & 7.95E0 & N/A & $6 / 22 / 09$ & 9E29002 \\
\hline 0902002-21 & B1YNF0 & $7.50 \mathrm{E} 0$ & N/A & $6 / 22 / 09$ & 9E29002 \\
\hline 0902002-22 & B1YNF1 & 7.49E0 & N/A & $6 / 22 / 09$ & 9E29002 \\
\hline $0902002-23$ & B1YD80 & $3.83 \mathrm{E} 1$ & N/A & $6 / 22 / 09$ & 9E29002 \\
\hline 0902002-24 & B1YD81 & $9.88 \mathrm{E} 0$ & N/A & $6 / 22 / 09$ & 9E29002 \\
\hline 0902002-25 & B1YD82 & $8.03 \mathrm{E} 1$ & $\mathrm{~N} / \mathrm{A}$ & $6 / 22 / 09$ & 9E29002 \\
\hline 0902002-26 & B1YNF2 & $1.92 \mathrm{E} 1$ & N/A & $6 / 22 / 09$ & 9E29002 \\
\hline 0902002-27 & B1YNF3 & $1.01 \mathrm{E} 1$ & N/A & $6 / 22 / 09$ & 9E29002 \\
\hline 0902002-28 & B1YNF4 & $1.87 \mathrm{E} 1$ & N/A & $6 / 22 / 09$ & 9E29002 \\
\hline 0902002-29 & B1YNF5 & $2.60 \mathrm{E} 1$ & N/A & $6 / 22 / 09$ & 9E29002 \\
\hline 0902002-30 & B1YNF6 & $9.16 \mathrm{E} 0$ & N/A & $6 / 22 / 09$ & 9E29002 \\
\hline 0902002-31 & B1YD83 & $1.82 \mathrm{E} 1$ & $\mathrm{~N} / \mathrm{A}$ & $6 / 22 / 09$ & 9E29002 \\
\hline 0902002-37 & B1YM97 & $1.25 \mathrm{E} 1$ & N/A & $2 / 10 / 10$ & 0B08009 \\
\hline
\end{tabular}


Anions by Ion Chromatography

\begin{tabular}{|c|c|c|c|c|c|c|c|}
\hline CAS \# & Analyte & Results & Units & EQL & Analyzed & Batch & Method \\
\hline HEIS No. & B1YD07 & \multicolumn{3}{|c|}{ Lab ID: $\quad$ 0902002-01 } & & & \\
\hline $16984-48-8$ & Fluoride & $5.50 \mathrm{E}-1$ & $\mathrm{ug} / \mathrm{g}$ dry & $2.16 \mathrm{E}-1$ & 3/10/09 & $9 \mathrm{C} 10004$ & AGG-IC-001 \\
\hline $16887-00-6$ & Chloride & $3.83 \mathrm{E} 0$ & $\mathrm{ug} / \mathrm{g}$ dry & $5.39 \mathrm{E}-1$ & $3 / 10 / 09$ & $9 \mathrm{C} 10004$ & AGG-IC-001 \\
\hline $14797-65-0$ & Nitrite & $<1.08 \mathrm{E} 0$ & $\mathrm{ug} / \mathrm{g}$ dry & $1.08 \mathrm{E} 0$ & $3 / 10 / 09$ & 9C10004 & AGG-IC-001 \\
\hline $14797-55-8$ & Nitrate & $2.92 \mathrm{E} 0$ & $\mathrm{ug} / \mathrm{g}$ dry & $1.08 \mathrm{E} 0$ & $3 / 10 / 09$ & 9C10004 & AGG-IC-001 \\
\hline $14808-79-8$ & Sulfate & $1.51 \mathrm{E} 1$ & $\mathrm{ug} / \mathrm{g}$ dry & $1.62 \mathrm{E} 0$ & 3/10/09 & $9 \mathrm{C} 10004$ & AGG-IC-001 \\
\hline $14265-44-2$ & Phosphate & $<1.62 \mathrm{E} 0$ & $\mathrm{ug} / \mathrm{g}$ dry & $1.62 \mathrm{E} 0$ & $3 / 10 / 09$ & 9C10004 & AGG-IC-001 \\
\hline HEIS No. & B1YD08 & \multicolumn{3}{|c|}{ Lab ID: $\quad 0902002-02$} & & & \\
\hline $16984-48-8$ & Fluoride & $2.06 \mathrm{E} 0$ & ug/g dry & $2.20 \mathrm{E}-1$ & $3 / 24 / 09$ & $9 \mathrm{C} 25004$ & AGG-IC-001 \\
\hline $16887-00-6$ & Chloride & $2.55 \mathrm{E} 0$ & $\mathrm{ug} / \mathrm{g}$ dry & $5.51 \mathrm{E}-1$ & $3 / 24 / 09$ & $9 \mathrm{C} 25004$ & AGG-IC-001 \\
\hline $14797-65-0$ & Nitrite & $<1.10 \mathrm{E} 0$ & ug/g dry & $1.10 \mathrm{E} 0$ & $3 / 24 / 09$ & $9 \mathrm{C} 25004$ & AGG-IC-001 \\
\hline $14797-55-8$ & Nitrate & $<1.10 \mathrm{E} 0$ & $\mathrm{ug} / \mathrm{g}$ dry & $1.10 \mathrm{E} 0$ & $3 / 24 / 09$ & $9 \mathrm{C} 25004$ & AGG-IC-001 \\
\hline $14808-79-8$ & Sulfate & 7.97E0 & ug/g dry & $1.65 \mathrm{E} 0$ & $3 / 24 / 09$ & $9 \mathrm{C} 25004$ & AGG-IC-001 \\
\hline $14265-44-2$ & Phosphate & $<1.65 \mathrm{E} 0$ & ug/g dry & $1.65 \mathrm{E} 0$ & $3 / 24 / 09$ & $9 \mathrm{C} 25004$ & AGG-IC-001 \\
\hline HEIS No. & B1YD09 & \multicolumn{3}{|c|}{ Lab ID: $\quad 0902002-03$} & & & \\
\hline $16984-48-8$ & Fluoride & $7.05 \mathrm{E}-1$ & ug/g dry & $2.22 \mathrm{E}-1$ & $3 / 24 / 09$ & $9 \mathrm{C} 25004$ & AGG-IC-001 \\
\hline $16887-00-6$ & Chloride & $2.65 \mathrm{E} 0$ & ug/g dry & $5.55 \mathrm{E}-1$ & $3 / 24 / 09$ & $9 \mathrm{C} 25004$ & AGG-IC-001 \\
\hline $14797-65-0$ & Nitrite & $<1.11 \mathrm{E} 0$ & $\mathrm{ug} / \mathrm{g}$ dry & $1.11 \mathrm{E} 0$ & $3 / 24 / 09$ & $9 \mathrm{C} 25004$ & AGG-IC-001 \\
\hline $14797-55-8$ & Nitrate & $8.70 \mathrm{E} 0$ & ug/g dry & $1.11 \mathrm{E} 0$ & $3 / 24 / 09$ & $9 \mathrm{C} 25004$ & AGG-IC-001 \\
\hline $14808-79-8$ & Sulfate & $4.96 \mathrm{E} 0$ & ug/g dry & $1.66 \mathrm{E} 0$ & $3 / 24 / 09$ & $9 \mathrm{C} 25004$ & AGG-IC-001 \\
\hline $14265-44-2$ & Phosphate & $<1.66 \mathrm{E} 0$ & ug/g dry & $1.66 \mathrm{E} 0$ & $3 / 24 / 09$ & $9 \mathrm{C} 25004$ & AGG-IC-001 \\
\hline HEIS No. & B1YND4 & \multicolumn{3}{|c|}{ Lab ID: $\quad 0902002-05$} & & & \\
\hline $16984-48-8$ & Fluoride & $3.21 \mathrm{E}-1$ & ug/g dry & $2.00 \mathrm{E}-1$ & $4 / 14 / 09$ & 9D14001 & AGG-IC-001 \\
\hline $16887-00-6$ & Chloride & 8.96E-1 & ug/g dry & $5.01 \mathrm{E}-1$ & $4 / 14 / 09$ & 9D14001 & AGG-IC-001 \\
\hline $14797-65-0$ & Nitrite & $<1.00 \mathrm{E} 0$ & ug/g dry & $1.00 \mathrm{E} 0$ & 4/14/09 & 9D14001 & AGG-IC-001 \\
\hline $14797-55-8$ & Nitrate & $2.01 \mathrm{E} 0$ & ug/g dry & $1.00 \mathrm{E} 0$ & 4/14/09 & 9D14001 & AGG-IC-001 \\
\hline $14808-79-8$ & Sulfate & $1.41 \mathrm{E} 1$ & $u g / g$ dry & $1.50 \mathrm{E} 0$ & $4 / 14 / 09$ & 9D14001 & AGG-IC-001 \\
\hline $14265-44-2$ & Phosphate & $<1.50 \mathrm{E} 0$ & ug/g dry & $1.50 \mathrm{E} 0$ & $4 / 14 / 09$ & 9D14001 & AGG-IC-001 \\
\hline HEIS No. & B1YND5 & \multicolumn{3}{|c|}{ Lab ID: $\quad 0902002-06$} & & & \\
\hline $16984-48-8$ & Fluoride & $5.67 \mathrm{E}-1$ & ug/g dry & $2.00 \mathrm{E}-1$ & $4 / 14 / 09$ & 9D14001 & AGG-IC-001 \\
\hline $16887-00-6$ & Chloride & $4.10 \mathrm{E} 0$ & $\mathrm{ug} / \mathrm{g}$ dry & $5.00 \mathrm{E}-1$ & $4 / 14 / 09$ & 9D14001 & AGG-IC-001 \\
\hline $14797-65-0$ & Nitrite & $<1.00 \mathrm{E} 0$ & $\mathrm{ug} / \mathrm{g}$ dry & $1.00 \mathrm{E} 0$ & $4 / 14 / 09$ & 9D14001 & AGG-IC-001 \\
\hline $14797-55-8$ & Nitrate & $<1.00 \mathrm{E} 0$ & ug/g dry & $1.00 \mathrm{E} 0$ & 4/14/09 & 9D14001 & AGG-IC-001 \\
\hline 14808-79-8 & Sulfate & $5.97 \mathrm{E} 0$ & ug/g dry & $1.50 \mathrm{E} 0$ & 4/14/09 & 9D14001 & AGG-IC-001 \\
\hline $14265-44-2$ & Phosphate & $<1.50 \mathrm{E} 0$ & ug/g dry & $1.50 \mathrm{E} 0$ & $4 / 14 / 09$ & 9D14001 & AGG-IC-001 \\
\hline HEIS No. & B1YND7 & \multicolumn{3}{|c|}{ Lab ID: $\quad$ 0902002-07 } & & & \\
\hline $16984-48-8$ & Fluoride & $1.28 \mathrm{E} 0$ & ug/g dry & $1.99 \mathrm{E}-1$ & $4 / 14 / 09$ & 9D14001 & AGG-IC-001 \\
\hline $16887-00-6$ & Chloride & $6.90 \mathrm{E} 0$ & ug/g dry & $4.98 \mathrm{E}-1$ & $4 / 14 / 09$ & 9D14001 & AGG-IC-001 \\
\hline $14797-65-0$ & Nitrite & $<9.96 \mathrm{E}-1$ & $\mathrm{ug} / \mathrm{g}$ dry & $9.96 \mathrm{E}-1$ & $4 / 14 / 09$ & 9D14001 & AGG-IC-001 \\
\hline $14797-55-8$ & Nitrate & $1.44 \mathrm{E} 0$ & $\mathrm{ug} / \mathrm{g}$ dry & $9.96 \mathrm{E}-1$ & 4/14/09 & 9D14001 & AGG-IC-001 \\
\hline $14808-79-8$ & Sulfate & $1.66 \mathrm{E} 1$ & ug/g dry & $1.49 \mathrm{E} 0$ & $4 / 14 / 09$ & 9D14001 & AGG-IC-001 \\
\hline $14265-44-2$ & Phosphate & $<1.49 \mathrm{E} 0$ & $\mathrm{ug} / \mathrm{g}$ dry & $1.49 \mathrm{E} 0$ & 4/14/09 & 9D14001 & AGG-IC-001 \\
\hline HEIS No. & B1YM90 & \multicolumn{3}{|c|}{ Lab ID: $\quad$ 0902002-08 } & & & \\
\hline $16984-48-8$ & Fluoride & $3.88 \mathrm{E}-1$ & ug/g dry & $2.00 \mathrm{E}-1$ & $4 / 14 / 09$ & 9D14001 & AGG-IC-001 \\
\hline $16887-00-6$ & Chloride & $2.72 \mathrm{E} 1$ & $u g / g$ dry & $5.00 \mathrm{E}-1$ & $4 / 14 / 09$ & 9D14001 & AGG-IC-001 \\
\hline $14797-65-0$ & Nitrite & $1.49 \mathrm{E} 0$ & ug/g dry & $1.00 \mathrm{E} 0$ & 4/14/09 & 9D14001 & AGG-IC-001 \\
\hline $14797-55-8$ & Nitrate & $5.54 \mathrm{E} 1$ & ug/g dry & $1.00 \mathrm{E} 0$ & $4 / 14 / 09$ & 9D14001 & AGG-IC-001 \\
\hline $14808-79-8$ & Sulfate & $5.39 \mathrm{E} 1$ & $\mathrm{ug} / \mathrm{g}$ dry & $1.50 \mathrm{E} 0$ & $4 / 14 / 09$ & 9D14001 & AGG-IC-001 \\
\hline $14265-44-2$ & Phosphate & $<1.50 \mathrm{E} 0$ & ug/g dry & $1.50 \mathrm{E} 0$ & 4/14/09 & 9D14001 & AGG-IC-001 \\
\hline
\end{tabular}




\section{Anions by Ion Chromatography}

\begin{tabular}{|c|c|c|c|c|c|c|c|}
\hline CAS \# & Analyte & Results & Units & EQL & Analyzed & Batch & Method \\
\hline HEIS No. & B1YM91 & \multicolumn{3}{|c|}{ Lab ID: $\quad 0902002-09$} & & & \\
\hline $16984-48-8$ & Fluoride & $5.31 \mathrm{E}-1$ & ug/g dry & $2.15 \mathrm{E}-1$ & $4 / 14 / 09$ & 9D14001 & AGG-IC-001 \\
\hline $16887-00-6$ & Chloride & $1.74 \mathrm{E} 0$ & ug/g dry & $5.36 \mathrm{E}-1$ & $4 / 14 / 09$ & 9D14001 & AGG-IC-001 \\
\hline $14797-65-0$ & Nitrite & $<1.07 \mathrm{E} 0$ & ug/g dry & $1.07 \mathrm{E} 0$ & $4 / 14 / 09$ & 9D14001 & AGG-IC-001 \\
\hline $14797-55-8$ & Nitrate & $2.51 \mathrm{E} 0$ & ug/g dry & $1.07 \mathrm{E} 0$ & $4 / 14 / 09$ & 9D14001 & AGG-IC-001 \\
\hline $14808-79-8$ & Sulfate & $6.17 \mathrm{E} 0$ & ug/g dry & $1.61 \mathrm{E} 0$ & $4 / 14 / 09$ & 9D14001 & AGG-IC-001 \\
\hline $14265-44-2$ & Phosphate & $<1.61 \mathrm{E} 0$ & ug/g dry & $1.61 \mathrm{E} 0$ & $4 / 14 / 09$ & 9D14001 & AGG-IC-001 \\
\hline HEIS No. & B1YM92 & \multicolumn{3}{|c|}{ Lab ID: $\quad 0902002-12$} & & & \\
\hline $16984-48-8$ & Fluoride & $5.59 \mathrm{E}-1$ & ug/g dry & $2.01 \mathrm{E}-1$ & $4 / 21 / 09$ & 9D21001 & AGG-IC-001 \\
\hline $16887-00-6$ & Chloride & $1.36 \mathrm{E} 1$ & ug/g dry & $5.03 \mathrm{E}-1$ & $4 / 21 / 09$ & 9D21001 & AGG-IC-001 \\
\hline $14797-65-0$ & Nitrite & $<1.01 \mathrm{E} 0$ & ug/g dry & $1.01 \mathrm{E} 0$ & $4 / 21 / 09$ & 9D21001 & AGG-IC-001 \\
\hline $14797-55-8$ & Nitrate & $3.91 \mathrm{E} 1$ & ug/g dry & $1.01 \mathrm{E} 0$ & $4 / 21 / 09$ & 9D21001 & AGG-IC-001 \\
\hline $14808-79-8$ & Sulfate & $2.23 \mathrm{E} 1$ & ug/g dry & $1.51 \mathrm{E} 0$ & $4 / 21 / 09$ & 9D21001 & AGG-IC-001 \\
\hline $14265-44-2$ & Phosphate & $<1.51 \mathrm{E} 0$ & ug/g dry & $1.51 \mathrm{E} 0$ & $4 / 21 / 09$ & 9D21001 & AGG-IC-001 \\
\hline
\end{tabular}


Total Metals by PNNL-AGG-ICP-AES/Water Extract

\begin{tabular}{|c|c|c|c|c|c|c|c|}
\hline CAS \# & Analyte & Results & Units & EQL & Analyzed & Batch & Method \\
\hline HEIS No. & B1YD07 & \multicolumn{3}{|c|}{ Lab ID: $\quad$ 0902002-01 } & & & \\
\hline $7429-90-5$ & Aluminum & $1.10 \mathrm{E}-1$ & ug/g dry & $1.01 \mathrm{E}-1$ & $5 / 13 / 09$ & 9E12002 & PNNL-AGG-ICP-AES \\
\hline $7440-42-8$ & Boron & $<1.48 \mathrm{E} 0$ & ug/g dry & $1.48 \mathrm{E} 0$ & $5 / 13 / 09$ & 9E12002 & PNNL-AGG-ICP-AES \\
\hline $7440-39-3$ & Barium & $<1.34 \mathrm{E}-1$ & ug/g dry & $1.34 \mathrm{E}-1$ & $5 / 13 / 09$ & 9E12002 & PNNL-AGG-ICP-AES \\
\hline $7440-41-7$ & Beryllium & $<1.42 \mathrm{E}-2$ & ug/g dry & $1.42 \mathrm{E}-2$ & $5 / 13 / 09$ & 9E12002 & PNNL-AGG-ICP-AES \\
\hline $7440-69-9$ & Bismuth & $<3.29 \mathrm{E}-1$ & ug/g dry & $3.29 \mathrm{E}-1$ & $5 / 13 / 09$ & 9E12002 & PNNL-AGG-ICP-AES \\
\hline $7440-70-2$ & Calcium & $1.11 \mathrm{E} 1$ & ug/g dry & $4.89 \mathrm{E}-1$ & $5 / 13 / 09$ & 9E12002 & PNNL-AGG-ICP-AES \\
\hline $7440-48-4$ & Cobalt & $<1.41 \mathrm{E}-1$ & ug/g dry & $1.41 \mathrm{E}-1$ & $5 / 13 / 09$ & 9E12002 & PNNL-AGG-ICP-AES \\
\hline $7440-50-8$ & Copper & $<9.49 \mathrm{E}-2$ & ug/g dry & $9.49 \mathrm{E}-2$ & $5 / 13 / 09$ & 9E12002 & PNNL-AGG-ICP-AES \\
\hline $7439-89-6$ & Iron & $<1.13 \mathrm{E}-1$ & ug/g dry & $1.13 \mathrm{E}-1$ & $5 / 13 / 09$ & 9E12002 & PNNL-AGG-ICP-AES \\
\hline $7440-09-7$ & Potassium & 4.40E0 & ug/g dry & $1.39 \mathrm{E} 0$ & $5 / 13 / 09$ & 9E12002 & PNNL-AGG-ICP-AES \\
\hline $7439-93-2$ & Lithium & $<8.07 \mathrm{E}-1$ & ug/g dry & 8.07E-1 & $5 / 13 / 09$ & 9E12002 & PNNL-AGG-ICP-AES \\
\hline $7439-95-4$ & Magnesium & $2.40 \mathrm{E} 0$ & ug/g dry & $5.28 \mathrm{E}-2$ & $5 / 13 / 09$ & 9E12002 & PNNL-AGG-ICP-AES \\
\hline $7439-96-5$ & Manganese & $<6.75 \mathrm{E}-2$ & ug/g dry & $6.75 \mathrm{E}-2$ & $5 / 13 / 09$ & 9E12002 & PNNL-AGG-ICP-AES \\
\hline $7439-98-7$ & Molybdenum & $<1.52 \mathrm{E}-1$ & ug/g dry & $1.52 \mathrm{E}-1$ & $5 / 13 / 09$ & 9E12002 & PNNL-AGG-ICP-AES \\
\hline $7723-14-0$ & Phosphorus & $<6.51 \mathrm{E}-1$ & ug/g dry & $6.51 \mathrm{E}-1$ & $5 / 13 / 09$ & 9E12002 & PNNL-AGG-ICP-AES \\
\hline $7440-24-6$ & Strontium & $<8.66 \mathrm{E}-2$ & ug/g dry & $8.66 \mathrm{E}-2$ & $5 / 13 / 09$ & 9E12002 & PNNL-AGG-ICP-AES \\
\hline $7440-62-2$ & Vanadium & $<1.44 \mathrm{E}-1$ & ug/g dry & 1.44E-1 & $5 / 13 / 09$ & 9E12002 & PNNL-AGG-ICP-AES \\
\hline $7440-66-6$ & Zinc & $<1.06 \mathrm{E}-1$ & ug/g dry & $1.06 \mathrm{E}-1$ & $5 / 13 / 09$ & 9E12002 & PNNL-AGG-ICP-AES \\
\hline $7440-23-5$ & Sodium & $1.26 \mathrm{E} 1$ & ug/g dry & $8.61 \mathrm{E}-1$ & $5 / 13 / 09$ & 9E12002 & PNNL-AGG-ICP-AES \\
\hline $7440-67-7$ & Zirconium & $<1.39 \mathrm{E}-1$ & ug/g dry & $1.39 \mathrm{E}-1$ & $5 / 13 / 09$ & 9E12002 & PNNL-AGG-ICP-AES \\
\hline HEIS No. & B1YD08 & \multicolumn{3}{|c|}{ Lab ID: $\quad$ 0902002-02 } & & & \\
\hline $7429-90-5$ & Aluminum & $3.13 \mathrm{E}-1$ & ug/g dry & $1.03 \mathrm{E}-1$ & $5 / 13 / 09$ & 9E12003 & PNNL-AGG-ICP-AES \\
\hline $7440-42-8$ & Boron & $<1.51 \mathrm{E} 0$ & ug/g dry & $1.51 \mathrm{E} 0$ & $5 / 13 / 09$ & 9E12003 & PNNL-AGG-ICP-AES \\
\hline $7440-39-3$ & Barium & $<1.36 \mathrm{E}-1$ & ug/g dry & $1.36 \mathrm{E}-1$ & $5 / 13 / 09$ & 9E12003 & PNNL-AGG-ICP-AES \\
\hline $7440-41-7$ & Beryllium & $<1.44 \mathrm{E}-2$ & ug/g dry & $1.44 \mathrm{E}-2$ & $5 / 13 / 09$ & 9E12003 & PNNL-AGG-ICP-AES \\
\hline $7440-69-9$ & Bismuth & $<3.35 \mathrm{E}-1$ & ug/g dry & $3.35 \mathrm{E}-1$ & $5 / 13 / 09$ & 9E12003 & PNNL-AGG-ICP-AES \\
\hline $7440-70-2$ & Calcium & 4.66Е0 & ug/g dry & $4.98 \mathrm{E}-1$ & $5 / 13 / 09$ & 9E12003 & PNNL-AGG-ICP-AES \\
\hline $7440-48-4$ & Cobalt & $<1.44 \mathrm{E}-1$ & ug/g dry & $1.44 \mathrm{E}-1$ & $5 / 13 / 09$ & 9E12003 & PNNL-AGG-ICP-AES \\
\hline $7440-50-8$ & Copper & $<9.67 \mathrm{E}-2$ & ug/g dry & $9.67 \mathrm{E}-2$ & $5 / 13 / 09$ & 9E12003 & PNNL-AGG-ICP-AES \\
\hline $7439-89-6$ & Iron & $3.91 \mathrm{E}-1$ & ug/g dry & $1.15 \mathrm{E}-1$ & $5 / 13 / 09$ & 9E12003 & PNNL-AGG-ICP-AES \\
\hline $7440-09-7$ & Potassium & $3.44 \mathrm{E} 0$ & ug/g dry & $1.42 \mathrm{E} 0$ & $5 / 13 / 09$ & 9E12003 & PNNL-AGG-ICP-AES \\
\hline $7439-93-2$ & Lithium & $<8.23 \mathrm{E}-1$ & ug/g dry & $8.23 \mathrm{E}-1$ & $5 / 13 / 09$ & 9E12003 & PNNL-AGG-ICP-AES \\
\hline $7439-95-4$ & Magnesium & $1.47 \mathrm{E} 0$ & ug/g dry & $5.38 \mathrm{E}-2$ & $5 / 13 / 09$ & 9E12003 & PNNL-AGG-ICP-AES \\
\hline $7439-96-5$ & Manganese & $<6.88 \mathrm{E}-2$ & ug/g dry & $6.88 \mathrm{E}-2$ & $5 / 13 / 09$ & 9E12003 & PNNL-AGG-ICP-AES \\
\hline $7439-98-7$ & Molybdenum & $<1.55 \mathrm{E}-1$ & ug/g dry & $1.55 \mathrm{E}-1$ & $5 / 13 / 09$ & 9E12003 & PNNL-AGG-ICP-AES \\
\hline $7723-14-0$ & Phosphorus & $<6.64 \mathrm{E}-1$ & ug/g dry & $6.64 \mathrm{E}-1$ & $5 / 13 / 09$ & 9E12003 & PNNL-AGG-ICP-AES \\
\hline $7440-24-6$ & Strontium & $<8.83 \mathrm{E}-2$ & ug/g dry & $8.83 \mathrm{E}-2$ & $5 / 13 / 09$ & 9E12003 & PNNL-AGG-ICP-AES \\
\hline $7440-62-2$ & Vanadium & $<1.47 \mathrm{E}-1$ & ug/g dry & $1.47 \mathrm{E}-1$ & $5 / 13 / 09$ & 9E12003 & PNNL-AGG-ICP-AES \\
\hline $7440-66-6$ & Zinc & $<1.08 \mathrm{E}-1$ & ug/g dry & $1.08 \mathrm{E}-1$ & $5 / 13 / 09$ & 9E12003 & PNNL-AGG-ICP-AES \\
\hline $7440-23-5$ & Sodium & $1.19 \mathrm{E} 1$ & ug/g dry & $8.78 \mathrm{E}-1$ & $5 / 13 / 09$ & 9E12003 & PNNL-AGG-ICP-AES \\
\hline $7440-67-7$ & Zirconium & $<1.42 \mathrm{E}-1$ & ug/g dry & $1.42 \mathrm{E}-1$ & $5 / 13 / 09$ & 9E12003 & PNNL-AGG-ICP-AES \\
\hline HEIS No. & B1YD09 & \multicolumn{3}{|c|}{ Lab ID: $\quad 0902002-03$} & & & \\
\hline $7429-90-5$ & Aluminum & $5.90 \mathrm{E}-1$ & ug/g dry & $1.04 \mathrm{E}-1$ & $5 / 13 / 09$ & 9E12003 & PNNL-AGG-ICP-AES \\
\hline $7440-42-8$ & Boron & $<1.52 \mathrm{E} 0$ & ug/g dry & $1.52 \mathrm{E} 0$ & $5 / 13 / 09$ & 9E12003 & PNNL-AGG-ICP-AES \\
\hline $7440-39-3$ & Barium & $<1.37 \mathrm{E}-1$ & ug/g dry & $1.37 \mathrm{E}-1$ & $5 / 13 / 09$ & 9E12003 & PNNL-AGG-ICP-AES \\
\hline $7440-41-7$ & Beryllium & $<1.45 \mathrm{E}-2$ & ug/g dry & $1.45 \mathrm{E}-2$ & $5 / 13 / 09$ & 9E12003 & PNNL-AGG-ICP-AES \\
\hline $7440-69-9$ & Bismuth & $<3.38 \mathrm{E}-1$ & ug/g dry & $3.38 \mathrm{E}-1$ & $5 / 13 / 09$ & 9E12003 & PNNL-AGG-ICP-AES \\
\hline $7440-70-2$ & Calcium & $2.39 \mathrm{E} 0$ & ug/g dry & $5.02 \mathrm{E}-1$ & $5 / 13 / 09$ & 9E12003 & PNNL-AGG-ICP-AES \\
\hline
\end{tabular}


Total Metals by PNNL-AGG-ICP-AES/Water Extract

\begin{tabular}{|c|c|c|c|c|c|c|c|}
\hline CAS \# & Analyte & Results & Units & EQL & Analyzed & Batch & Method \\
\hline HEIS No. & B1YD09 & \multicolumn{3}{|c|}{ Lab ID: $\quad 0902002-03$} & & & \\
\hline $7440-48-4$ & Cobalt & $<1.45 \mathrm{E}-1$ & ug/g dry & $1.45 \mathrm{E}-1$ & $5 / 13 / 09$ & 9E12003 & PNNL-AGG-ICP-AES \\
\hline $7440-50-8$ & Copper & $<9.74 \mathrm{E}-2$ & ug/g dry & $9.74 \mathrm{E}-2$ & $5 / 13 / 09$ & 9E12003 & PNNL-AGG-ICP-AES \\
\hline $7439-89-6$ & Iron & $7.38 \mathrm{E}-1$ & $\mathrm{ug} / \mathrm{g}$ dry & $1.16 \mathrm{E}-1$ & $5 / 13 / 09$ & 9E12003 & PNNL-AGG-ICP-AES \\
\hline 7440-09-7 & Potassium & $2.18 \mathrm{E} 0$ & ug/g dry & $1.43 \mathrm{E} 0$ & $5 / 13 / 09$ & 9E12003 & PNNL-AGG-ICP-AES \\
\hline $7439-93-2$ & Lithium & $<8.28 \mathrm{E}-1$ & ug/g dry & $8.28 \mathrm{E}-1$ & $5 / 13 / 09$ & 9E12003 & PNNL-AGG-ICP-AES \\
\hline $7439-95-4$ & Magnesium & $8.08 \mathrm{E}-1$ & ug/g dry & $5.42 \mathrm{E}-2$ & $5 / 13 / 09$ & 9E12003 & PNNL-AGG-ICP-AES \\
\hline $7439-96-5$ & Manganese & $<6.93 \mathrm{E}-2$ & ug/g dry & $6.93 \mathrm{E}-2$ & $5 / 13 / 09$ & 9E12003 & PNNL-AGG-ICP-AES \\
\hline $7439-98-7$ & Molybdenum & $<1.56 \mathrm{E}-1$ & ug/g dry & $1.56 \mathrm{E}-1$ & $5 / 13 / 09$ & 9E12003 & PNNL-AGG-ICP-AES \\
\hline $7723-14-0$ & Phosphorus & $<6.69 \mathrm{E}-1$ & ug/g dry & $6.69 \mathrm{E}-1$ & $5 / 13 / 09$ & 9E12003 & PNNL-AGG-ICP-AES \\
\hline $7440-24-6$ & Strontium & $<8.89 \mathrm{E}-2$ & ug/g dry & $8.89 \mathrm{E}-2$ & $5 / 13 / 09$ & 9E12003 & PNNL-AGG-ICP-AES \\
\hline $7440-62-2$ & Vanadium & $<1.48 \mathrm{E}-1$ & ug/g dry & $1.48 \mathrm{E}-1$ & $5 / 13 / 09$ & 9E12003 & PNNL-AGG-ICP-AES \\
\hline $7440-66-6$ & Zinc & $<1.08 \mathrm{E}-1$ & ug/g dry & $1.08 \mathrm{E}-1$ & $5 / 13 / 09$ & 9E12003 & PNNL-AGG-ICP-AES \\
\hline $7440-23-5$ & Sodium & $1.13 \mathrm{E} 1$ & ug/g dry & $8.84 \mathrm{E}-1$ & $5 / 13 / 09$ & 9E12003 & PNNL-AGG-ICP-AES \\
\hline $7440-67-7$ & Zirconium & $<1.43 \mathrm{E}-1$ & ug/g dry & $1.43 \mathrm{E}-1$ & $5 / 13 / 09$ & 9E12003 & PNNL-AGG-ICP-AES \\
\hline HEIS No. & B1YND4 & \multicolumn{3}{|c|}{ Lab ID: $\quad$ 0902002-05 } & & & \\
\hline $7429-90-5$ & Aluminum & $<9.38 \mathrm{E}-2$ & ug/g dry & $9.38 \mathrm{E}-2$ & $5 / 13 / 09$ & 9E12004 & PNNL-AGG-ICP-AES \\
\hline $7440-42-8$ & Boron & $<1.37 \mathrm{E} 0$ & ug/g dry & $1.37 \mathrm{E} 0$ & $5 / 13 / 09$ & 9E12004 & PNNL-AGG-ICP-AES \\
\hline $7440-39-3$ & Barium & $<1.24 \mathrm{E}-1$ & ug/g dry & $1.24 \mathrm{E}-1$ & $5 / 13 / 09$ & 9E12004 & PNNL-AGG-ICP-AES \\
\hline $7440-41-7$ & Beryllium & $<1.31 \mathrm{E}-2$ & ug/g dry & $1.31 \mathrm{E}-2$ & $5 / 13 / 09$ & 9E12004 & PNNL-AGG-ICP-AES \\
\hline $7440-69-9$ & Bismuth & $<3.05 \mathrm{E}-1$ & ug/g dry & $3.05 \mathrm{E}-1$ & $5 / 13 / 09$ & 9E12004 & PNNL-AGG-ICP-AES \\
\hline $7440-70-2$ & Calcium & $9.02 \mathrm{E} 0$ & ug/g dry & $4.53 \mathrm{E}-1$ & $5 / 13 / 09$ & 9E12004 & PNNL-AGG-ICP-AES \\
\hline $7440-48-4$ & Cobalt & $<1.31 \mathrm{E}-1$ & ug/g dry & $1.31 \mathrm{E}-1$ & $5 / 13 / 09$ & 9E12004 & PNNL-AGG-ICP-AES \\
\hline $7440-50-8$ & Copper & $<8.79 \mathrm{E}-2$ & ug/g dry & $8.79 \mathrm{E}-2$ & $5 / 13 / 09$ & 9E12004 & PNNL-AGG-ICP-AES \\
\hline $7439-89-6$ & Iron & $<1.05 \mathrm{E}-1$ & ug/g dry & $1.05 \mathrm{E}-1$ & $5 / 13 / 09$ & 9E12004 & PNNL-AGG-ICP-AES \\
\hline 7440-09-7 & Potassium & $2.82 \mathrm{E} 0$ & ug/g dry & $1.29 \mathrm{E} 0$ & $5 / 13 / 09$ & 9E12004 & PNNL-AGG-ICP-AES \\
\hline $7439-93-2$ & Lithium & $<7.48 \mathrm{E}-1$ & ug/g dry & $7.48 \mathrm{E}-1$ & $5 / 13 / 09$ & 9E12004 & PNNL-AGG-ICP-AES \\
\hline $7439-95-4$ & Magnesium & $2.48 \mathrm{E} 0$ & ug/g dry & $4.89 \mathrm{E}-2$ & $5 / 13 / 09$ & 9E12004 & PNNL-AGG-ICP-AES \\
\hline $7439-96-5$ & Manganese & $<6.25 \mathrm{E}-2$ & ug/g dry & $6.25 \mathrm{E}-2$ & $5 / 13 / 09$ & 9E12004 & PNNL-AGG-ICP-AES \\
\hline $7439-98-7$ & Molybdenum & $<1.41 \mathrm{E}-1$ & ug/g dry & $1.41 \mathrm{E}-1$ & $5 / 13 / 09$ & 9E12004 & PNNL-AGG-ICP-AES \\
\hline $7723-14-0$ & Phosphorus & $1.74 \mathrm{E} 0$ & ug/g dry & $6.04 \mathrm{E}-1$ & $5 / 13 / 09$ & 9E12004 & PNNL-AGG-ICP-AES \\
\hline $7440-24-6$ & Strontium & $<8.03 \mathrm{E}-2$ & ug/g dry & $8.03 \mathrm{E}-2$ & $5 / 13 / 09$ & 9E12004 & PNNL-AGG-ICP-AES \\
\hline $7440-62-2$ & Vanadium & $<1.33 \mathrm{E}-1$ & ug/g dry & $1.33 \mathrm{E}-1$ & $5 / 13 / 09$ & 9E12004 & PNNL-AGG-ICP-AES \\
\hline $7440-66-6$ & Zinc & $<9.79 \mathrm{E}-2$ & ug/g dry & $9.79 \mathrm{E}-2$ & $5 / 13 / 09$ & 9E12004 & PNNL-AGG-ICP-AES \\
\hline $7440-23-5$ & Sodium & $8.18 \mathrm{E} 0$ & ug/g dry & $7.98 \mathrm{E}-1$ & $5 / 13 / 09$ & 9E12004 & PNNL-AGG-ICP-AES \\
\hline $7440-67-7$ & Zirconium & $<1.29 \mathrm{E}-1$ & ug/g dry & $1.29 \mathrm{E}-1$ & $5 / 13 / 09$ & 9E12004 & PNNL-AGG-ICP-AES \\
\hline HEIS No. & B1YND5 & \multicolumn{3}{|c|}{ Lab ID: $\quad 0902002-06$} & & & \\
\hline $7429-90-5$ & Aluminum & $5.26 \mathrm{E}-1$ & ug/g dry & $9.37 \mathrm{E}-2$ & $5 / 13 / 09$ & 9E12004 & PNNL-AGG-ICP-AES \\
\hline $7440-42-8$ & Boron & $<1.37 \mathrm{E} 0$ & ug/g dry & $1.37 \mathrm{E} 0$ & $5 / 13 / 09$ & 9E12004 & PNNL-AGG-ICP-AES \\
\hline $7440-39-3$ & Barium & $<1.24 \mathrm{E}-1$ & ug/g dry & $1.24 \mathrm{E}-1$ & $5 / 13 / 09$ & 9E12004 & PNNL-AGG-ICP-AES \\
\hline $7440-41-7$ & Beryllium & $<1.31 \mathrm{E}-2$ & ug/g dry & $1.31 \mathrm{E}-2$ & $5 / 13 / 09$ & 9E12004 & PNNL-AGG-ICP-AES \\
\hline $7440-69-9$ & Bismuth & $<3.04 \mathrm{E}-1$ & ug/g dry & $3.04 \mathrm{E}-1$ & $5 / 13 / 09$ & 9E12004 & PNNL-AGG-ICP-AES \\
\hline $7440-70-2$ & Calcium & $8.14 \mathrm{E} 0$ & $\mathrm{ug} / \mathrm{g}$ dry & $4.52 \mathrm{E}-1$ & $5 / 13 / 09$ & 9E12004 & PNNL-AGG-ICP-AES \\
\hline $7440-48-4$ & Cobalt & $<1.31 \mathrm{E}-1$ & ug/g dry & $1.31 \mathrm{E}-1$ & $5 / 13 / 09$ & 9E12004 & PNNL-AGG-ICP-AES \\
\hline $7440-50-8$ & Copper & $<8.79 \mathrm{E}-2$ & ug/g dry & $8.79 \mathrm{E}-2$ & $5 / 13 / 09$ & 9E12004 & PNNL-AGG-ICP-AES \\
\hline $7439-89-6$ & Iron & $4.80 \mathrm{E}-1$ & ug/g dry & $1.05 \mathrm{E}-1$ & $5 / 13 / 09$ & 9E12004 & PNNL-AGG-ICP-AES \\
\hline $7440-09-7$ & Potassium & $3.51 \mathrm{E} 0$ & $\mathrm{ug} / \mathrm{g}$ dry & $1.29 \mathrm{E} 0$ & $5 / 13 / 09$ & 9E12004 & PNNL-AGG-ICP-AES \\
\hline $7439-93-2$ & Lithium & $<7.47 \mathrm{E}-1$ & ug/g dry & 7.47E-1 & $5 / 13 / 09$ & 9E12004 & PNNL-AGG-ICP-AES \\
\hline $7439-95-4$ & Magnesium & 3.03E0 & ug/g dry & $4.89 \mathrm{E}-2$ & $5 / 13 / 09$ & 9E12004 & PNNL-AGG-ICP-AES \\
\hline
\end{tabular}


Total Metals by PNNL-AGG-ICP-AES/Water Extract

\begin{tabular}{|c|c|c|c|c|c|c|c|}
\hline CAS \# & Analyte & Results & Units & EQL & Analyzed & Batch & Method \\
\hline HEIS No. & B1YND5 & \multicolumn{3}{|c|}{ Lab ID: $\quad$ 0902002-06 } & & & \\
\hline $7439-96-5$ & Manganese & $<6.25 \mathrm{E}-2$ & ug/g dry & $6.25 \mathrm{E}-2$ & $5 / 13 / 09$ & 9E12004 & PNNL-AGG-ICP-AES \\
\hline 7439-98-7 & Molybdenum & $<1.41 \mathrm{E}-1$ & ug/g dry & $1.41 \mathrm{E}-1$ & $5 / 13 / 09$ & 9E12004 & PNNL-AGG-ICP-AES \\
\hline $7723-14-0$ & Phosphorus & $<6.03 \mathrm{E}-1$ & $\mathrm{ug} / \mathrm{g}$ dry & $6.03 \mathrm{E}-1$ & $5 / 13 / 09$ & 9E12004 & PNNL-AGG-ICP-AES \\
\hline $7440-24-6$ & Strontium & $<8.02 \mathrm{E}-2$ & ug/g dry & $8.02 \mathrm{E}-2$ & $5 / 13 / 09$ & 9E12004 & PNNL-AGG-ICP-AES \\
\hline $7440-62-2$ & Vanadium & $<1.33 \mathrm{E}-1$ & ug/g dry & $1.33 \mathrm{E}-1$ & $5 / 13 / 09$ & 9E12004 & PNNL-AGG-ICP-AES \\
\hline $7440-66-6$ & Zinc & $<9.78 \mathrm{E}-2$ & ug/g dry & $9.78 \mathrm{E}-2$ & $5 / 13 / 09$ & 9E12004 & PNNL-AGG-ICP-AES \\
\hline $7440-23-5$ & Sodium & $1.22 \mathrm{E} 1$ & ug/g dry & $7.97 \mathrm{E}-1$ & $5 / 13 / 09$ & 9E12004 & PNNL-AGG-ICP-AES \\
\hline $7440-67-7$ & Zirconium & $<1.29 \mathrm{E}-1$ & ug/g dry & $1.29 \mathrm{E}-1$ & $5 / 13 / 09$ & 9E12004 & PNNL-AGG-ICP-AES \\
\hline HEIS No. & B1YND7 & \multicolumn{3}{|c|}{ Lab ID: $\quad$ 0902002-07 } & & & \\
\hline $7429-90-5$ & Aluminum & $3.29 \mathrm{E}-1$ & ug/g dry & $9.34 \mathrm{E}-2$ & $5 / 13 / 09$ & 9E12004 & PNNL-AGG-ICP-AES \\
\hline $7440-42-8$ & Boron & $<1.36 \mathrm{E} 0$ & ug/g dry & $1.36 \mathrm{E} 0$ & $5 / 13 / 09$ & 9E12004 & PNNL-AGG-ICP-AES \\
\hline $7440-39-3$ & Barium & $<1.23 \mathrm{E}-1$ & ug/g dry & $1.23 \mathrm{E}-1$ & $5 / 13 / 09$ & 9E12004 & PNNL-AGG-ICP-AES \\
\hline $7440-41-7$ & Beryllium & $<1.31 \mathrm{E}-2$ & ug/g dry & $1.31 \mathrm{E}-2$ & $5 / 13 / 09$ & 9E12004 & PNNL-AGG-ICP-AES \\
\hline $7440-69-9$ & Bismuth & $<3.03 \mathrm{E}-1$ & ug/g dry & $3.03 \mathrm{E}-1$ & $5 / 13 / 09$ & 9E12004 & PNNL-AGG-ICP-AES \\
\hline $7440-70-2$ & Calcium & $6.42 \mathrm{E} 0$ & ug/g dry & $4.51 \mathrm{E}-1$ & $5 / 13 / 09$ & 9E12004 & PNNL-AGG-ICP-AES \\
\hline $7440-48-4$ & Cobalt & $<1.30 \mathrm{E}-1$ & ug/g dry & $1.30 \mathrm{E}-1$ & $5 / 13 / 09$ & 9E12004 & PNNL-AGG-ICP-AES \\
\hline $7440-50-8$ & Copper & $<8.75 \mathrm{E}-2$ & ug/g dry & $8.75 \mathrm{E}-2$ & $5 / 13 / 09$ & 9E12004 & PNNL-AGG-ICP-AES \\
\hline $7439-89-6$ & Iron & $3.08 \mathrm{E}-1$ & ug/g dry & $1.04 \mathrm{E}-1$ & $5 / 13 / 09$ & 9E12004 & PNNL-AGG-ICP-AES \\
\hline $7440-09-7$ & Potassium & $4.62 \mathrm{E} 0$ & ug/g dry & $1.29 \mathrm{E} 0$ & $5 / 13 / 09$ & 9E12004 & PNNL-AGG-ICP-AES \\
\hline $7439-93-2$ & Lithium & $<7.44 \mathrm{E}-1$ & ug/g dry & 7.44E-1 & $5 / 13 / 09$ & 9E12004 & PNNL-AGG-ICP-AES \\
\hline $7439-95-4$ & Magnesium & $2.19 \mathrm{E} 0$ & ug/g dry & $4.87 \mathrm{E}-2$ & $5 / 13 / 09$ & 9E12004 & PNNL-AGG-ICP-AES \\
\hline $7439-96-5$ & Manganese & $<6.22 \mathrm{E}-2$ & ug/g dry & $6.22 \mathrm{E}-2$ & $5 / 13 / 09$ & 9E12004 & PNNL-AGG-ICP-AES \\
\hline $7439-98-7$ & Molybdenum & $<1.40 \mathrm{E}-1$ & ug/g dry & $1.40 \mathrm{E}-1$ & $5 / 13 / 09$ & 9E12004 & PNNL-AGG-ICP-AES \\
\hline $7723-14-0$ & Phosphorus & $<6.01 \mathrm{E}-1$ & ug/g dry & $6.01 \mathrm{E}-1$ & $5 / 13 / 09$ & 9E12004 & PNNL-AGG-ICP-AES \\
\hline $7440-24-6$ & Strontium & $<7.99 \mathrm{E}-2$ & ug/g dry & $7.99 \mathrm{E}-2$ & $5 / 13 / 09$ & 9E12004 & PNNL-AGG-ICP-AES \\
\hline $7440-62-2$ & Vanadium & $<1.33 \mathrm{E}-1$ & ug/g dry & $1.33 \mathrm{E}-1$ & $5 / 13 / 09$ & 9E12004 & PNNL-AGG-ICP-AES \\
\hline $7440-66-6$ & Zinc & $<9.74 \mathrm{E}-2$ & ug/g dry & $9.74 \mathrm{E}-2$ & $5 / 13 / 09$ & 9E12004 & PNNL-AGG-ICP-AES \\
\hline $7440-23-5$ & Sodium & $2.23 \mathrm{E} 1$ & ug/g dry & 7.94E-1 & $5 / 13 / 09$ & 9E12004 & PNNL-AGG-ICP-AES \\
\hline $7440-67-7$ & Zirconium & $<1.28 \mathrm{E}-1$ & ug/g dry & $1.28 \mathrm{E}-1$ & $5 / 13 / 09$ & 9E12004 & PNNL-AGG-ICP-AES \\
\hline HEIS No. & B1YM90 & \multicolumn{3}{|c|}{ Lab ID: $\quad$ 0902002-08 } & & & \\
\hline $7429-90-5$ & Aluminum & $<9.38 \mathrm{E}-2$ & ug/g dry & $9.38 \mathrm{E}-2$ & $5 / 13 / 09$ & 9E12004 & PNNL-AGG-ICP-AES \\
\hline $7440-42-8$ & Boron & $<1.37 \mathrm{E} 0$ & ug/g dry & $1.37 \mathrm{E} 0$ & $5 / 13 / 09$ & 9E12004 & PNNL-AGG-ICP-AES \\
\hline $7440-39-3$ & Barium & $<1.24 \mathrm{E}-1$ & ug/g dry & $1.24 \mathrm{E}-1$ & $5 / 13 / 09$ & 9E12004 & PNNL-AGG-ICP-AES \\
\hline $7440-41-7$ & Beryllium & $<1.31 \mathrm{E}-2$ & ug/g dry & $1.31 \mathrm{E}-2$ & $5 / 13 / 09$ & 9E12004 & PNNL-AGG-ICP-AES \\
\hline $7440-69-9$ & Bismuth & $<3.04 \mathrm{E}-1$ & ug/g dry & $3.04 \mathrm{E}-1$ & $5 / 13 / 09$ & 9E12004 & PNNL-AGG-ICP-AES \\
\hline $7440-70-2$ & Calcium & $5.45 \mathrm{E} 1$ & ug/g dry & $4.52 \mathrm{E}-1$ & $5 / 13 / 09$ & 9E12004 & PNNL-AGG-ICP-AES \\
\hline $7440-48-4$ & Cobalt & $<1.31 \mathrm{E}-1$ & ug/g dry & $1.31 \mathrm{E}-1$ & $5 / 13 / 09$ & 9E12004 & PNNL-AGG-ICP-AES \\
\hline $7440-50-8$ & Copper & $<8.79 \mathrm{E}-2$ & ug/g dry & $8.79 \mathrm{E}-2$ & $5 / 13 / 09$ & 9E12004 & PNNL-AGG-ICP-AES \\
\hline $7439-89-6$ & Iron & $<1.05 \mathrm{E}-1$ & ug/g dry & $1.05 \mathrm{E}-1$ & $5 / 13 / 09$ & 9E12004 & PNNL-AGG-ICP-AES \\
\hline $7440-09-7$ & Potassium & $6.38 \mathrm{E} 0$ & ug/g dry & $1.29 \mathrm{E} 0$ & $5 / 13 / 09$ & 9E12004 & PNNL-AGG-ICP-AES \\
\hline $7439-93-2$ & Lithium & $<7.47 \mathrm{E}-1$ & ug/g dry & $7.47 \mathrm{E}-1$ & $5 / 13 / 09$ & 9E12004 & PNNL-AGG-ICP-AES \\
\hline $7439-95-4$ & Magnesium & $1.17 \mathrm{E} 1$ & ug/g dry & $4.89 \mathrm{E}-2$ & $5 / 13 / 09$ & 9E12004 & PNNL-AGG-ICP-AES \\
\hline $7439-96-5$ & Manganese & $<6.25 \mathrm{E}-2$ & ug/g dry & $6.25 \mathrm{E}-2$ & $5 / 13 / 09$ & 9E12004 & PNNL-AGG-ICP-AES \\
\hline 7439-98-7 & Molybdenum & $<1.41 \mathrm{E}-1$ & ug/g dry & $1.41 \mathrm{E}-1$ & $5 / 13 / 09$ & 9E12004 & PNNL-AGG-ICP-AES \\
\hline $7723-14-0$ & Phosphorus & $1.47 \mathrm{E} 1$ & ug/g dry & $6.03 \mathrm{E}-1$ & $5 / 13 / 09$ & 9E12004 & PNNL-AGG-ICP-AES \\
\hline $7440-24-6$ & Strontium & $2.54 \mathrm{E}-1$ & ug/g dry & $8.02 \mathrm{E}-2$ & $5 / 13 / 09$ & 9E12004 & PNNL-AGG-ICP-AES \\
\hline $7440-62-2$ & Vanadium & $<1.33 \mathrm{E}-1$ & ug/g dry & $1.33 \mathrm{E}-1$ & $5 / 13 / 09$ & 9E12004 & PNNL-AGG-ICP-AES \\
\hline $7440-66-6$ & Zinc & $<9.78 \mathrm{E}-2$ & ug/g dry & $9.78 \mathrm{E}-2$ & $5 / 13 / 09$ & 9E12004 & PNNL-AGG-ICP-AES \\
\hline
\end{tabular}


Total Metals by PNNL-AGG-ICP-AES/Water Extract

\begin{tabular}{|c|c|c|c|c|c|c|c|}
\hline CAS \# & Analyte & Results & Units & EQL & Analyzed & Batch & Method \\
\hline HEIS No. & B1YM90 & \multicolumn{3}{|c|}{ Lab ID: $\quad 0902002-08$} & & & \\
\hline $7440-23-5$ & Sodium & $2.84 \mathrm{E} 1$ & $u g / g$ dry & $7.98 \mathrm{E}-1$ & $5 / 13 / 09$ & 9E12004 & PNNL-AGG-ICP-AES \\
\hline $7440-67-7$ & Zirconium & $<1.29 \mathrm{E}-1$ & ug/g dry & $1.29 \mathrm{E}-1$ & $5 / 13 / 09$ & 9E12004 & PNNL-AGG-ICP-AES \\
\hline HEIS No. & B1YM91 & \multicolumn{3}{|c|}{ Lab ID: $\quad$ 0902002-09 } & & & \\
\hline $7429-90-5$ & Aluminum & $3.83 \mathrm{E}-1$ & ug/g dry & $1.01 \mathrm{E}-1$ & $5 / 13 / 09$ & 9E12004 & PNNL-AGG-ICP-AES \\
\hline $7440-42-8$ & Boron & $<1.47 \mathrm{E} 0$ & ug/g dry & $1.47 \mathrm{E} 0$ & $5 / 13 / 09$ & 9E12004 & PNNL-AGG-ICP-AES \\
\hline $7440-39-3$ & Barium & $<1.33 \mathrm{E}-1$ & $u g / g$ dry & $1.33 \mathrm{E}-1$ & $5 / 13 / 09$ & 9E12004 & PNNL-AGG-ICP-AES \\
\hline $7440-41-7$ & Beryllium & $<1.41 \mathrm{E}-2$ & ug/g dry & $1.41 \mathrm{E}-2$ & $5 / 13 / 09$ & 9E12004 & PNNL-AGG-ICP-AES \\
\hline $7440-69-9$ & Bismuth & $<3.26 \mathrm{E}-1$ & ug/g dry & $3.26 \mathrm{E}-1$ & $5 / 13 / 09$ & 9E12004 & PNNL-AGG-ICP-AES \\
\hline $7440-70-2$ & Calcium & $8.33 \mathrm{E} 0$ & $\mathrm{ug} / \mathrm{g}$ dry & $4.85 \mathrm{E}-1$ & $5 / 13 / 09$ & 9E12004 & PNNL-AGG-ICP-AES \\
\hline $7440-48-4$ & Cobalt & $<1.40 \mathrm{E}-1$ & ug/g dry & $1.40 \mathrm{E}-1$ & $5 / 13 / 09$ & 9E12004 & PNNL-AGG-ICP-AES \\
\hline $7440-50-8$ & Copper & $<9.42 \mathrm{E}-2$ & ug/g dry & $9.42 \mathrm{E}-2$ & $5 / 13 / 09$ & 9E12004 & PNNL-AGG-ICP-AES \\
\hline $7439-89-6$ & Iron & $3.68 \mathrm{E}-1$ & ug/g dry & $1.12 \mathrm{E}-1$ & $5 / 13 / 09$ & 9E12004 & PNNL-AGG-ICP-AES \\
\hline $7440-09-7$ & Potassium & $3.32 \mathrm{E} 0$ & ug/g dry & $1.39 \mathrm{E} 0$ & $5 / 13 / 09$ & 9E12004 & PNNL-AGG-ICP-AES \\
\hline $7439-93-2$ & Lithium & $<8.01 \mathrm{E}-1$ & ug/g dry & $8.01 \mathrm{E}-1$ & $5 / 13 / 09$ & 9E12004 & PNNL-AGG-ICP-AES \\
\hline $7439-95-4$ & Magnesium & 2.73E0 & ug/g dry & $5.24 \mathrm{E}-2$ & $5 / 13 / 09$ & 9E12004 & PNNL-AGG-ICP-AES \\
\hline $7439-96-5$ & Manganese & $<6.70 \mathrm{E}-2$ & ug/g dry & $6.70 \mathrm{E}-2$ & $5 / 13 / 09$ & 9E12004 & PNNL-AGG-ICP-AES \\
\hline 7439-98-7 & Molybdenum & $<1.51 \mathrm{E}-1$ & $\mathrm{ug} / \mathrm{g}$ dry & $1.51 \mathrm{E}-1$ & $5 / 13 / 09$ & 9E12004 & PNNL-AGG-ICP-AES \\
\hline 7723-14-0 & Phosphorus & $<6.47 \mathrm{E}-1$ & ug/g dry & $6.47 \mathrm{E}-1$ & $5 / 13 / 09$ & 9E12004 & PNNL-AGG-ICP-AES \\
\hline $7440-24-6$ & Strontium & $<8.60 \mathrm{E}-2$ & ug/g dry & $8.60 \mathrm{E}-2$ & $5 / 13 / 09$ & 9E12004 & PNNL-AGG-ICP-AES \\
\hline $7440-62-2$ & Vanadium & $<1.43 \mathrm{E}-1$ & ug/g dry & $1.43 \mathrm{E}-1$ & $5 / 13 / 09$ & 9E12004 & PNNL-AGG-ICP-AES \\
\hline $7440-66-6$ & Zinc & $<1.05 \mathrm{E}-1$ & ug/g dry & $1.05 \mathrm{E}-1$ & $5 / 13 / 09$ & 9E12004 & PNNL-AGG-ICP-AES \\
\hline $7440-23-5$ & Sodium & $9.43 \mathrm{E} 0$ & ug/g dry & $8.55 \mathrm{E}-1$ & $5 / 13 / 09$ & 9E12004 & PNNL-AGG-ICP-AES \\
\hline $7440-67-7$ & Zirconium & $<1.38 \mathrm{E}-1$ & ug/g dry & $1.38 \mathrm{E}-1$ & $5 / 13 / 09$ & 9E12004 & PNNL-AGG-ICP-AES \\
\hline HEIS No. & B1YM92 & \multicolumn{3}{|c|}{ Lab ID: $\quad 0902002-12$} & & & \\
\hline $7429-90-5$ & Aluminum & $3.10 \mathrm{E}-1$ & ug/g dry & $9.43 \mathrm{E}-2$ & $5 / 13 / 09$ & 9E12005 & PNNL-AGG-ICP-AES \\
\hline $7440-42-8$ & Boron & $<1.38 \mathrm{E} 0$ & ug/g dry & $1.38 \mathrm{E} 0$ & $5 / 13 / 09$ & 9E12005 & PNNL-AGG-ICP-AES \\
\hline $7440-39-3$ & Barium & $<1.25 \mathrm{E}-1$ & ug/g dry & $1.25 \mathrm{E}-1$ & $5 / 13 / 09$ & 9E12005 & PNNL-AGG-ICP-AES \\
\hline $7440-41-7$ & Beryllium & $<1.32 \mathrm{E}-2$ & ug/g dry & $1.32 \mathrm{E}-2$ & $5 / 13 / 09$ & 9E12005 & PNNL-AGG-ICP-AES \\
\hline $7440-69-9$ & Bismuth & $<3.06 \mathrm{E}-1$ & $\mathrm{ug} / \mathrm{g}$ dry & $3.06 \mathrm{E}-1$ & $5 / 13 / 09$ & 9E12005 & PNNL-AGG-ICP-AES \\
\hline $7440-70-2$ & Calcium & $1.98 \mathrm{E} 1$ & ug/g dry & $4.55 \mathrm{E}-1$ & $5 / 13 / 09$ & 9E12005 & PNNL-AGG-ICP-AES \\
\hline $7440-48-4$ & Cobalt & $<1.32 \mathrm{E}-1$ & ug/g dry & $1.32 \mathrm{E}-1$ & $5 / 13 / 09$ & 9E12005 & PNNL-AGG-ICP-AES \\
\hline $7440-50-8$ & Copper & $<8.84 \mathrm{E}-2$ & ug/g dry & $8.84 \mathrm{E}-2$ & $5 / 13 / 09$ & 9E12005 & PNNL-AGG-ICP-AES \\
\hline $7439-89-6$ & Iron & $<1.06 \mathrm{E}-1$ & ug/g dry & $1.06 \mathrm{E}-1$ & $5 / 13 / 09$ & 9E12005 & PNNL-AGG-ICP-AES \\
\hline 7440-09-7 & Potassium & $5.99 \mathrm{E} 0$ & ug/g dry & $1.30 \mathrm{E} 0$ & $5 / 13 / 09$ & 9E12005 & PNNL-AGG-ICP-AES \\
\hline $7439-93-2$ & Lithium & $<7.52 \mathrm{E}-1$ & ug/g dry & $7.52 \mathrm{E}-1$ & $5 / 13 / 09$ & 9E12005 & PNNL-AGG-ICP-AES \\
\hline $7439-95-4$ & Magnesium & $5.99 \mathrm{E} 0$ & $\mathrm{ug} / \mathrm{g}$ dry & $4.92 \mathrm{E}-2$ & $5 / 13 / 09$ & 9E12005 & PNNL-AGG-ICP-AES \\
\hline $7439-96-5$ & Manganese & $<6.29 \mathrm{E}-2$ & ug/g dry & $6.29 \mathrm{E}-2$ & $5 / 13 / 09$ & 9E12005 & PNNL-AGG-ICP-AES \\
\hline 7439-98-7 & Molybdenum & $<1.41 \mathrm{E}-1$ & ug/g dry & $1.41 \mathrm{E}-1$ & $5 / 13 / 09$ & 9E12005 & PNNL-AGG-ICP-AES \\
\hline $7723-14-0$ & Phosphorus & $<6.07 \mathrm{E}-1$ & ug/g dry & $6.07 \mathrm{E}-1$ & $5 / 13 / 09$ & 9E12005 & PNNL-AGG-ICP-AES \\
\hline $7440-24-6$ & Strontium & $9.00 \mathrm{E}-2$ & ug/g dry & $8.07 \mathrm{E}-2$ & $5 / 13 / 09$ & 9E12005 & PNNL-AGG-ICP-AES \\
\hline $7440-62-2$ & Vanadium & $<1.34 \mathrm{E}-1$ & ug/g dry & $1.34 \mathrm{E}-1$ & $5 / 13 / 09$ & 9E12005 & PNNL-AGG-ICP-AES \\
\hline $7440-66-6$ & Zinc & $<9.84 \mathrm{E}-2$ & ug/g dry & $9.84 \mathrm{E}-2$ & $5 / 13 / 09$ & 9E12005 & PNNL-AGG-ICP-AES \\
\hline $7440-23-5$ & Sodium & $1.67 \mathrm{E} 1$ & ug/g dry & $8.02 \mathrm{E}-1$ & $5 / 13 / 09$ & 9E12005 & PNNL-AGG-ICP-AES \\
\hline $7440-67-7$ & Zirconium & $<1.29 \mathrm{E}-1$ & ug/g dry & $1.29 \mathrm{E}-1$ & $5 / 13 / 09$ & 9E12005 & PNNL-AGG-ICP-AES \\
\hline
\end{tabular}


Total Metals by PNNL-AGG-ICP-AES/Acid Extract

\begin{tabular}{|c|c|c|c|c|c|c|c|}
\hline CAS \# & Analyte & Results & Units & EQL & Analyzed & Batch & Method \\
\hline HEIS No. & B1YD07 & \multicolumn{3}{|c|}{ Lab ID: $\quad$ 0902002-01 } & & & \\
\hline $7429-90-5$ & Aluminum & $5.29 \mathrm{E} 3$ & $\mathrm{ug} / \mathrm{g}$ dry & $2.32 \mathrm{E} 1$ & $5 / 12 / 09$ & 9E12001 & PNNL-AGG-ICP-AES \\
\hline $7440-42-8$ & Boron & $<9.93 \mathrm{E} 1$ & $\mathrm{ug} / \mathrm{g}$ dry & 9.93E1 & $5 / 12 / 09$ & 9E12001 & PNNL-AGG-ICP-AES \\
\hline $7440-39-3$ & Barium & $5.29 \mathrm{E} 1$ & $\mathrm{ug} / \mathrm{g}$ dry & $1.41 \mathrm{E} 0$ & $5 / 12 / 09$ & 9E12001 & PNNL-AGG-ICP-AES \\
\hline $7440-41-7$ & Beryllium & $<7.92 \mathrm{E}-1$ & $\mathrm{ug} / \mathrm{g}$ dry & 7.92E-1 & $5 / 12 / 09$ & 9E12001 & PNNL-AGG-ICP-AES \\
\hline $7440-69-9$ & Bismuth & $<9.76 \mathrm{E} 0$ & $\mathrm{ug} / \mathrm{g}$ dry & $9.76 \mathrm{E} 0$ & $5 / 12 / 09$ & 9E12001 & PNNL-AGG-ICP-AES \\
\hline $7440-70-2$ & Calcium & $2.72 \mathrm{E} 3$ & $\mathrm{ug} / \mathrm{g}$ dry & $2.41 \mathrm{E} 1$ & $5 / 12 / 09$ & 9E12001 & PNNL-AGG-ICP-AES \\
\hline $7440-48-4$ & Cobalt & $6.30 \mathrm{E} 0$ & ug/g dry & $4.95 \mathrm{E} 0$ & $5 / 12 / 09$ & 9E12001 & PNNL-AGG-ICP-AES \\
\hline $7440-50-8$ & Copper & $7.91 \mathrm{E} 0$ & $\mathrm{ug} / \mathrm{g}$ dry & $6.63 \mathrm{E} 0$ & $5 / 12 / 09$ & 9E12001 & PNNL-AGG-ICP-AES \\
\hline $7439-89-6$ & Iron & $1.61 \mathrm{E} 4$ & ug/g dry & $6.23 \mathrm{E} 0$ & $5 / 12 / 09$ & 9E12001 & PNNL-AGG-ICP-AES \\
\hline $7440-09-7$ & Potassium & $6.36 \mathrm{E} 2$ & $\mathrm{ug} / \mathrm{g}$ dry & $8.87 \mathrm{E} 1$ & $5 / 12 / 09$ & 9E12001 & PNNL-AGG-ICP-AES \\
\hline $7439-93-2$ & Lithium & $<2.15 \mathrm{E} 1$ & $u g / g$ dry & $2.15 \mathrm{E} 1$ & $5 / 12 / 09$ & 9E12001 & PNNL-AGG-ICP-AES \\
\hline $7439-95-4$ & Magnesium & $3.03 \mathrm{E} 3$ & $\mathrm{ug} / \mathrm{g}$ dry & $6.60 \mathrm{E} 0$ & $5 / 12 / 09$ & 9E12001 & PNNL-AGG-ICP-AES \\
\hline $7439-96-5$ & Manganese & $2.02 \mathrm{E} 2$ & ug/g dry & $2.07 \mathrm{E} 0$ & $5 / 12 / 09$ & 9E12001 & PNNL-AGG-ICP-AES \\
\hline $7439-98-7$ & Molybdenum & $<7.87 \mathrm{E} 0$ & $\mathrm{ug} / \mathrm{g}$ dry & $7.87 \mathrm{E} 0$ & $5 / 12 / 09$ & 9E12001 & PNNL-AGG-ICP-AES \\
\hline $7723-14-0$ & Phosphorus & $2.85 \mathrm{E} 2$ & ug/g dry & $3.06 \mathrm{E} 1$ & $5 / 12 / 09$ & 9E12001 & PNNL-AGG-ICP-AES \\
\hline $7440-24-6$ & Strontium & $1.60 \mathrm{E} 1$ & $\mathrm{ug} / \mathrm{g}$ dry & $1.51 \mathrm{E} 0$ & $5 / 12 / 09$ & 9E12001 & PNNL-AGG-ICP-AES \\
\hline $7440-62-2$ & Vanadium & $2.18 \mathrm{E} 1$ & ug/g dry & $4.14 \mathrm{E} 0$ & $5 / 12 / 09$ & 9E12001 & PNNL-AGG-ICP-AES \\
\hline $7440-66-6$ & Zinc & $2.86 \mathrm{E} 1$ & $\mathrm{ug} / \mathrm{g}$ dry & $3.78 \mathrm{E} 0$ & $5 / 12 / 09$ & 9E12001 & PNNL-AGG-ICP-AES \\
\hline $7440-23-5$ & Sodium & $2.87 \mathrm{E} 2$ & ug/g dry & $6.53 \mathrm{E} 1$ & $5 / 12 / 09$ & 9E12001 & PNNL-AGG-ICP-AES \\
\hline $7440-67-7$ & Zirconium & $6.02 \mathrm{E} 0$ & ug/g dry & $2.77 \mathrm{E} 0$ & $5 / 12 / 09$ & 9E12001 & PNNL-AGG-ICP-AES \\
\hline HEIS No. & B1YD08 & \multicolumn{3}{|c|}{ Lab ID: $\quad$ 0902002-02 } & & & \\
\hline $7429-90-5$ & Aluminum & $9.25 \mathrm{E} 3$ & ug/g dry & $2.40 \mathrm{E} 1$ & $5 / 12 / 09$ & 9E12001 & PNNL-AGG-ICP-AES \\
\hline $7440-42-8$ & Boron & $<1.03 \mathrm{E} 2$ & ug/g dry & $1.03 \mathrm{E} 2$ & $5 / 12 / 09$ & 9E12001 & PNNL-AGG-ICP-AES \\
\hline $7440-39-3$ & Barium & $8.50 \mathrm{E} 1$ & $\mathrm{ug} / \mathrm{g}$ dry & $1.46 \mathrm{E} 0$ & $5 / 12 / 09$ & 9E12001 & PNNL-AGG-ICP-AES \\
\hline $7440-41-7$ & Beryllium & $<8.21 \mathrm{E}-1$ & ug/g dry & $8.21 \mathrm{E}-1$ & $5 / 12 / 09$ & 9E12001 & PNNL-AGG-ICP-AES \\
\hline $7440-69-9$ & Bismuth & $1.78 \mathrm{E} 1$ & ug/g dry & $1.01 \mathrm{E} 1$ & $5 / 12 / 09$ & 9E12001 & PNNL-AGG-ICP-AES \\
\hline $7440-70-2$ & Calcium & $1.18 \mathrm{E} 4$ & ug/g dry & $2.49 \mathrm{E} 1$ & $5 / 12 / 09$ & 9E12001 & PNNL-AGG-ICP-AES \\
\hline $7440-48-4$ & Cobalt & $9.08 \mathrm{E} 0$ & $\mathrm{ug} / \mathrm{g}$ dry & $5.13 \mathrm{E} 0$ & $5 / 12 / 09$ & 9E12001 & PNNL-AGG-ICP-AES \\
\hline $7440-50-8$ & Copper & $9.28 \mathrm{E} 0$ & ug/g dry & $6.87 \mathrm{E} 0$ & $5 / 12 / 09$ & 9E12001 & PNNL-AGG-ICP-AES \\
\hline $7439-89-6$ & Iron & $1.98 \mathrm{E} 4$ & ug/g dry & $6.46 \mathrm{E} 0$ & $5 / 12 / 09$ & 9E12001 & PNNL-AGG-ICP-AES \\
\hline $7440-09-7$ & Potassium & $1.78 \mathrm{E} 3$ & $\mathrm{ug} / \mathrm{g}$ dry & $9.19 \mathrm{E} 1$ & $5 / 12 / 09$ & 9E12001 & PNNL-AGG-ICP-AES \\
\hline $7439-93-2$ & Lithium & $<2.23 \mathrm{E} 1$ & $\mathrm{ug} / \mathrm{g}$ dry & $2.23 \mathrm{E} 1$ & $5 / 12 / 09$ & 9E12001 & PNNL-AGG-ICP-AES \\
\hline $7439-95-4$ & Magnesium & $5.52 \mathrm{E} 3$ & ug/g dry & $6.84 \mathrm{E} 0$ & $5 / 12 / 09$ & 9E12001 & PNNL-AGG-ICP-AES \\
\hline $7439-96-5$ & Manganese & $3.48 \mathrm{E} 2$ & ug/g dry & $2.15 \mathrm{E} 0$ & $5 / 12 / 09$ & 9E12001 & PNNL-AGG-ICP-AES \\
\hline $7439-98-7$ & Molybdenum & $<8.15 \mathrm{E} 0$ & $\mathrm{ug} / \mathrm{g}$ dry & $8.15 \mathrm{E} 0$ & $5 / 12 / 09$ & 9E12001 & PNNL-AGG-ICP-AES \\
\hline $7723-14-0$ & Phosphorus & $6.05 \mathrm{E} 2$ & ug/g dry & $3.17 \mathrm{E} 1$ & $5 / 12 / 09$ & 9E12001 & PNNL-AGG-ICP-AES \\
\hline $7440-24-6$ & Strontium & $4.26 \mathrm{E} 1$ & ug/g dry & $1.56 \mathrm{E} 0$ & $5 / 12 / 09$ & 9E12001 & PNNL-AGG-ICP-AES \\
\hline $7440-62-2$ & Vanadium & $3.02 \mathrm{E} 1$ & $\mathrm{ug} / \mathrm{g}$ dry & $4.30 \mathrm{E} 0$ & $5 / 12 / 09$ & 9E12001 & PNNL-AGG-ICP-AES \\
\hline $7440-66-6$ & Zinc & $4.16 \mathrm{E} 1$ & $\mathrm{ug} / \mathrm{g}$ dry & $3.92 \mathrm{E} 0$ & $5 / 12 / 09$ & 9E12001 & PNNL-AGG-ICP-AES \\
\hline $7440-23-5$ & Sodium & $2.29 \mathrm{E} 2$ & ug/g dry & $6.77 \mathrm{E} 1$ & $5 / 12 / 09$ & 9E12001 & PNNL-AGG-ICP-AES \\
\hline $7440-67-7$ & Zirconium & $1.38 \mathrm{E} 1$ & $\mathrm{ug} / \mathrm{g}$ dry & $2.88 \mathrm{E} 0$ & $5 / 12 / 09$ & 9E12001 & PNNL-AGG-ICP-AES \\
\hline HEIS No. & B1YD09 & \multicolumn{3}{|c|}{ Lab ID: $\quad 0902002-03$} & & & \\
\hline $7429-90-5$ & Aluminum & $7.12 \mathrm{E} 3$ & ug/g dry & $2.38 \mathrm{E} 1$ & $5 / 12 / 09$ & 9E12001 & PNNL-AGG-ICP-AES \\
\hline $7440-42-8$ & Boron & $<1.02 \mathrm{E} 2$ & ug/g dry & $1.02 \mathrm{E} 2$ & $5 / 12 / 09$ & 9E12001 & PNNL-AGG-ICP-AES \\
\hline $7440-39-3$ & Barium & $7.89 \mathrm{E} 1$ & ug/g dry & $1.45 \mathrm{E} 0$ & $5 / 12 / 09$ & 9E12001 & PNNL-AGG-ICP-AES \\
\hline $7440-41-7$ & Beryllium & $<8.14 \mathrm{E}-1$ & ug/g dry & $8.14 \mathrm{E}-1$ & $5 / 12 / 09$ & 9E12001 & PNNL-AGG-ICP-AES \\
\hline $7440-69-9$ & Bismuth & $<1.00 \mathrm{E} 1$ & $\mathrm{ug} / \mathrm{g}$ dry & $1.00 \mathrm{E} 1$ & $5 / 12 / 09$ & 9E12001 & PNNL-AGG-ICP-AES \\
\hline $7440-70-2$ & Calcium & $3.08 \mathrm{E} 3$ & ug/g dry & $2.47 \mathrm{E} 1$ & $5 / 12 / 09$ & 9E12001 & PNNL-AGG-ICP-AES \\
\hline
\end{tabular}


Total Metals by PNNL-AGG-ICP-AES/Acid Extract

\begin{tabular}{|c|c|c|c|c|c|c|c|}
\hline CAS \# & Analyte & Results & Units & EQL & Analyzed & Batch & Method \\
\hline HEIS No. & B1YD09 & \multicolumn{3}{|c|}{ Lab ID: $\quad 0902002-03$} & & & \\
\hline $7440-48-4$ & Cobalt & $7.31 \mathrm{E} 0$ & $\mathrm{ug} / \mathrm{g}$ dry & $5.09 \mathrm{E} 0$ & $5 / 12 / 09$ & 9E12001 & PNNL-AGG-ICP-AES \\
\hline $7440-50-8$ & Copper & $<6.81 \mathrm{E} 0$ & $\mathrm{ug} / \mathrm{g}$ dry & $6.81 \mathrm{E} 0$ & $5 / 12 / 09$ & 9E12001 & PNNL-AGG-ICP-AES \\
\hline $7439-89-6$ & Iron & $1.85 \mathrm{E} 4$ & $\mathrm{ug} / \mathrm{g}$ dry & $6.41 \mathrm{E} 0$ & $5 / 12 / 09$ & 9E12001 & PNNL-AGG-ICP-AES \\
\hline $7440-09-7$ & Potassium & $1.73 \mathrm{E} 3$ & $\mathrm{ug} / \mathrm{g}$ dry & $9.12 \mathrm{E} 1$ & $5 / 12 / 09$ & 9E12001 & PNNL-AGG-ICP-AES \\
\hline $7439-93-2$ & Lithium & $<2.21 \mathrm{E} 1$ & $\mathrm{ug} / \mathrm{g}$ dry & $2.21 \mathrm{E} 1$ & $5 / 12 / 09$ & 9E12001 & PNNL-AGG-ICP-AES \\
\hline $7439-95-4$ & Magnesium & $3.50 \mathrm{E} 3$ & $\mathrm{ug} / \mathrm{g}$ dry & $6.78 \mathrm{E} 0$ & $5 / 12 / 09$ & 9E12001 & PNNL-AGG-ICP-AES \\
\hline $7439-96-5$ & Manganese & $6.80 \mathrm{E} 2$ & ug/g dry & 2.13E0 & $5 / 12 / 09$ & 9E12001 & PNNL-AGG-ICP-AES \\
\hline $7439-98-7$ & Molybdenum & $<8.09 \mathrm{E} 0$ & $\mathrm{ug} / \mathrm{g}$ dry & $8.09 \mathrm{E} 0$ & $5 / 12 / 09$ & 9E12001 & PNNL-AGG-ICP-AES \\
\hline $7723-14-0$ & Phosphorus & $3.42 \mathrm{E} 2$ & ug/g dry & $3.15 \mathrm{E} 1$ & $5 / 12 / 09$ & 9E12001 & PNNL-AGG-ICP-AES \\
\hline $7440-24-6$ & Strontium & $1.91 \mathrm{E} 1$ & $\mathrm{ug} / \mathrm{g}$ dry & $1.55 \mathrm{E} 0$ & $5 / 12 / 09$ & 9E12001 & PNNL-AGG-ICP-AES \\
\hline $7440-62-2$ & Vanadium & $2.25 \mathrm{E} 1$ & $u g / g$ dry & $4.26 \mathrm{E} 0$ & $5 / 12 / 09$ & 9E12001 & PNNL-AGG-ICP-AES \\
\hline $7440-66-6$ & Zinc & $5.25 \mathrm{E} 1$ & $\mathrm{ug} / \mathrm{g}$ dry & $3.88 \mathrm{E} 0$ & $5 / 12 / 09$ & 9E12001 & PNNL-AGG-ICP-AES \\
\hline $7440-23-5$ & Sodium & $2.37 \mathrm{E} 2$ & $u g / g$ dry & $6.71 \mathrm{E} 1$ & $5 / 12 / 09$ & 9E12001 & PNNL-AGG-ICP-AES \\
\hline $7440-67-7$ & Zirconium & 8.69E0 & ug/g dry & $2.85 \mathrm{E} 0$ & $5 / 12 / 09$ & 9E12001 & PNNL-AGG-ICP-AES \\
\hline HEIS No. & B1YND4 & \multicolumn{3}{|c|}{ Lab ID: $\quad$ 0902002-05 } & & & \\
\hline $7429-90-5$ & Aluminum & $7.57 \mathrm{E} 3$ & $\mathrm{ug} / \mathrm{g}$ dry & $2.29 \mathrm{E} 1$ & $5 / 12 / 09$ & 9E12001 & PNNL-AGG-ICP-AES \\
\hline $7440-42-8$ & Boron & $<9.84 \mathrm{E} 1$ & ug/g dry & $9.84 \mathrm{E} 1$ & $5 / 12 / 09$ & 9E12001 & PNNL-AGG-ICP-AES \\
\hline $7440-39-3$ & Barium & $5.60 \mathrm{E} 1$ & ug/g dry & $1.40 \mathrm{E} 0$ & $5 / 12 / 09$ & 9E12001 & PNNL-AGG-ICP-AES \\
\hline $7440-41-7$ & Beryllium & $<7.84 \mathrm{E}-1$ & ug/g dry & $7.84 \mathrm{E}-1$ & $5 / 12 / 09$ & 9E12001 & PNNL-AGG-ICP-AES \\
\hline $7440-69-9$ & Bismuth & $1.74 \mathrm{E} 1$ & $\mathrm{ug} / \mathrm{g}$ dry & $9.67 \mathrm{E} 0$ & $5 / 12 / 09$ & 9E12001 & PNNL-AGG-ICP-AES \\
\hline $7440-70-2$ & Calcium & $8.45 \mathrm{E} 3$ & ug/g dry & $2.38 \mathrm{E} 1$ & $5 / 12 / 09$ & 9E12001 & PNNL-AGG-ICP-AES \\
\hline $7440-48-4$ & Cobalt & $6.92 \mathrm{E} 0$ & $\mathrm{ug} / \mathrm{g}$ dry & $4.90 \mathrm{E} 0$ & $5 / 12 / 09$ & 9E12001 & PNNL-AGG-ICP-AES \\
\hline $7440-50-8$ & Copper & 7.69E0 & ug/g dry & $6.56 \mathrm{E} 0$ & $5 / 12 / 09$ & 9E12001 & PNNL-AGG-ICP-AES \\
\hline $7439-89-6$ & Iron & $1.75 \mathrm{E} 4$ & $\mathrm{ug} / \mathrm{g}$ dry & $6.17 \mathrm{E} 0$ & $5 / 12 / 09$ & 9E12001 & PNNL-AGG-ICP-AES \\
\hline $7440-09-7$ & Potassium & $1.30 \mathrm{E} 3$ & ug/g dry & $8.78 \mathrm{E} 1$ & $5 / 12 / 09$ & 9E12001 & PNNL-AGG-ICP-AES \\
\hline $7439-93-2$ & Lithium & $<2.13 \mathrm{E} 1$ & ug/g dry & $2.13 \mathrm{E} 1$ & $5 / 12 / 09$ & 9E12001 & PNNL-AGG-ICP-AES \\
\hline $7439-95-4$ & Magnesium & $5.30 \mathrm{E} 3$ & ug/g dry & $6.53 \mathrm{E} 0$ & $5 / 12 / 09$ & 9E12001 & PNNL-AGG-ICP-AES \\
\hline $7439-96-5$ & Manganese & $2.81 \mathrm{E} 2$ & $\mathrm{ug} / \mathrm{g}$ dry & $2.05 \mathrm{E} 0$ & $5 / 12 / 09$ & 9E12001 & PNNL-AGG-ICP-AES \\
\hline $7439-98-7$ & Molybdenum & $<7.79 \mathrm{E} 0$ & ug/g dry & 7.79E0 & $5 / 12 / 09$ & 9E12001 & PNNL-AGG-ICP-AES \\
\hline $7723-14-0$ & Phosphorus & $4.52 \mathrm{E} 2$ & ug/g dry & 3.03E1 & $5 / 12 / 09$ & 9E12001 & PNNL-AGG-ICP-AES \\
\hline $7440-24-6$ & Strontium & $3.40 \mathrm{E} 1$ & $\mathrm{ug} / \mathrm{g}$ dry & $1.50 \mathrm{E} 0$ & $5 / 12 / 09$ & 9E12001 & PNNL-AGG-ICP-AES \\
\hline $7440-62-2$ & Vanadium & $2.48 \mathrm{E} 1$ & $\mathrm{ug} / \mathrm{g}$ dry & $4.10 \mathrm{E} 0$ & $5 / 12 / 09$ & 9E12001 & PNNL-AGG-ICP-AES \\
\hline $7440-66-6$ & Zinc & $3.34 \mathrm{E} 1$ & ug/g dry & $3.74 \mathrm{E} 0$ & $5 / 12 / 09$ & 9E12001 & PNNL-AGG-ICP-AES \\
\hline $7440-23-5$ & Sodium & $1.74 \mathrm{E} 2$ & ug/g dry & $6.46 \mathrm{E} 1$ & $5 / 12 / 09$ & 9E12001 & PNNL-AGG-ICP-AES \\
\hline $7440-67-7$ & Zirconium & $4.76 \mathrm{E} 0$ & ug/g dry & $2.75 \mathrm{E} 0$ & $5 / 12 / 09$ & 9E12001 & PNNL-AGG-ICP-AES \\
\hline HEIS No. & B1YND5 & \multicolumn{3}{|c|}{ Lab ID: $\quad 0902002-06$} & & & \\
\hline $7429-90-5$ & Aluminum & $6.40 \mathrm{E} 3$ & ug/g dry & $2.43 \mathrm{E} 1$ & $5 / 12 / 09$ & 9E12001 & PNNL-AGG-ICP-AES \\
\hline $7440-42-8$ & Boron & $<1.04 \mathrm{E} 2$ & ug/g dry & $1.04 \mathrm{E} 2$ & $5 / 12 / 09$ & 9E12001 & PNNL-AGG-ICP-AES \\
\hline $7440-39-3$ & Barium & $5.91 \mathrm{E} 1$ & ug/g dry & $1.48 \mathrm{E} 0$ & $5 / 12 / 09$ & 9E12001 & PNNL-AGG-ICP-AES \\
\hline $7440-41-7$ & Beryllium & $<8.30 \mathrm{E}-1$ & $\mathrm{ug} / \mathrm{g}$ dry & $8.30 \mathrm{E}-1$ & $5 / 12 / 09$ & 9E12001 & PNNL-AGG-ICP-AES \\
\hline $7440-69-9$ & Bismuth & $1.03 \mathrm{E} 1$ & ug/g dry & $1.02 \mathrm{E} 1$ & $5 / 12 / 09$ & 9E12001 & PNNL-AGG-ICP-AES \\
\hline $7440-70-2$ & Calcium & $3.89 \mathrm{E} 3$ & ug/g dry & $2.52 \mathrm{E} 1$ & $5 / 12 / 09$ & 9E12001 & PNNL-AGG-ICP-AES \\
\hline $7440-48-4$ & Cobalt & 8.69E0 & ug/g dry & $5.19 \mathrm{E} 0$ & $5 / 12 / 09$ & 9E12001 & PNNL-AGG-ICP-AES \\
\hline $7440-50-8$ & Copper & $9.19 \mathrm{E} 0$ & ug/g dry & $6.95 \mathrm{E} 0$ & $5 / 12 / 09$ & 9E12001 & PNNL-AGG-ICP-AES \\
\hline $7439-89-6$ & Iron & $1.80 \mathrm{E} 4$ & ug/g dry & $6.53 \mathrm{E} 0$ & $5 / 12 / 09$ & 9E12001 & PNNL-AGG-ICP-AES \\
\hline 7440-09-7 & Potassium & $5.87 \mathrm{E} 2$ & $u g / g$ dry & $9.30 \mathrm{E} 1$ & $5 / 12 / 09$ & 9E12001 & PNNL-AGG-ICP-AES \\
\hline $7439-93-2$ & Lithium & $<2.26 \mathrm{E} 1$ & $\mathrm{ug} / \mathrm{g}$ dry & $2.26 \mathrm{E} 1$ & $5 / 12 / 09$ & 9E12001 & PNNL-AGG-ICP-AES \\
\hline $7439-95-4$ & Magnesium & $3.86 \mathrm{E} 3$ & ug/g dry & $6.92 \mathrm{E} 0$ & $5 / 12 / 09$ & 9E12001 & PNNL-AGG-ICP-AES \\
\hline
\end{tabular}


Total Metals by PNNL-AGG-ICP-AES/Acid Extract

\begin{tabular}{|c|c|c|c|c|c|c|c|}
\hline CAS \# & Analyte & Results & Units & EQL & Analyzed & Batch & Method \\
\hline HEIS No. & B1YND5 & \multicolumn{3}{|c|}{ Lab ID: $\quad 0902002-06$} & & & \\
\hline $7439-96-5$ & Manganese & $2.29 \mathrm{E} 2$ & $\mathrm{ug} / \mathrm{g}$ dry & $2.17 \mathrm{E} 0$ & $5 / 12 / 09$ & 9E12001 & PNNL-AGG-ICP-AES \\
\hline $7439-98-7$ & Molybdenum & $<8.25 \mathrm{E} 0$ & $\mathrm{ug} / \mathrm{g}$ dry & $8.25 \mathrm{E} 0$ & $5 / 12 / 09$ & 9E12001 & PNNL-AGG-ICP-AES \\
\hline $7723-14-0$ & Phosphorus & $3.88 \mathrm{E} 2$ & $\mathrm{ug} / \mathrm{g}$ dry & $3.21 \mathrm{E} 1$ & $5 / 12 / 09$ & 9E12001 & PNNL-AGG-ICP-AES \\
\hline $7440-24-6$ & Strontium & $1.94 \mathrm{E} 1$ & $\mathrm{ug} / \mathrm{g}$ dry & $1.58 \mathrm{E} 0$ & $5 / 12 / 09$ & 9E12001 & PNNL-AGG-ICP-AES \\
\hline $7440-62-2$ & Vanadium & $2.99 \mathrm{E} 1$ & $\mathrm{ug} / \mathrm{g}$ dry & $4.35 \mathrm{E} 0$ & $5 / 12 / 09$ & 9E12001 & PNNL-AGG-ICP-AES \\
\hline $7440-66-6$ & Zinc & $3.27 \mathrm{E} 1$ & $\mathrm{ug} / \mathrm{g}$ dry & $3.96 \mathrm{E} 0$ & $5 / 12 / 09$ & 9E12001 & PNNL-AGG-ICP-AES \\
\hline $7440-23-5$ & Sodium & $2.85 \mathrm{E} 2$ & ug/g dry & $6.84 \mathrm{E} 1$ & $5 / 12 / 09$ & 9E12001 & PNNL-AGG-ICP-AES \\
\hline $7440-67-7$ & Zirconium & 8.67E0 & ug/g dry & $2.91 \mathrm{E} 0$ & $5 / 12 / 09$ & 9E12001 & PNNL-AGG-ICP-AES \\
\hline HEIS No. & B1YND7 & \multicolumn{3}{|c|}{ Lab ID: $\quad$ 0902002-07 } & & & \\
\hline $7429-90-5$ & Aluminum & $8.72 \mathrm{E} 3$ & ug/g dry & $2.39 \mathrm{E} 1$ & $5 / 12 / 09$ & 9E12001 & PNNL-AGG-ICP-AES \\
\hline $7440-42-8$ & Boron & $<1.02 \mathrm{E} 2$ & $\mathrm{ug} / \mathrm{g}$ dry & $1.02 \mathrm{E} 2$ & $5 / 12 / 09$ & 9E12001 & PNNL-AGG-ICP-AES \\
\hline $7440-39-3$ & Barium & $1.23 \mathrm{E} 2$ & ug/g dry & $1.45 \mathrm{E} 0$ & $5 / 12 / 09$ & 9E12001 & PNNL-AGG-ICP-AES \\
\hline $7440-41-7$ & Beryllium & $<8.16 \mathrm{E}-1$ & ug/g dry & $8.16 \mathrm{E}-1$ & $5 / 12 / 09$ & 9E12001 & PNNL-AGG-ICP-AES \\
\hline $7440-69-9$ & Bismuth & $<1.01 \mathrm{E} 1$ & ug/g dry & $1.01 \mathrm{E} 1$ & $5 / 12 / 09$ & 9E12001 & PNNL-AGG-ICP-AES \\
\hline $7440-70-2$ & Calcium & $4.62 \mathrm{E} 3$ & ug/g dry & $2.48 \mathrm{E} 1$ & $5 / 12 / 09$ & 9E12001 & PNNL-AGG-ICP-AES \\
\hline $7440-48-4$ & Cobalt & $8.75 \mathrm{E} 0$ & ug/g dry & $5.10 \mathrm{E} 0$ & $5 / 12 / 09$ & 9E12001 & PNNL-AGG-ICP-AES \\
\hline $7440-50-8$ & Copper & $2.30 \mathrm{E} 1$ & ug/g dry & $6.83 \mathrm{E} 0$ & $5 / 12 / 09$ & 9E12001 & PNNL-AGG-ICP-AES \\
\hline $7439-89-6$ & Iron & $2.04 \mathrm{E} 4$ & ug/g dry & $6.42 \mathrm{E} 0$ & $5 / 12 / 09$ & 9E12001 & PNNL-AGG-ICP-AES \\
\hline $7440-09-7$ & Potassium & $1.09 \mathrm{E} 3$ & $\mathrm{ug} / \mathrm{g}$ dry & $9.14 \mathrm{E} 1$ & $5 / 12 / 09$ & 9E12001 & PNNL-AGG-ICP-AES \\
\hline $7439-93-2$ & Lithium & $<2.22 \mathrm{E} 1$ & $\mathrm{ug} / \mathrm{g}$ dry & $2.22 \mathrm{E} 1$ & $5 / 12 / 09$ & 9E12001 & PNNL-AGG-ICP-AES \\
\hline $7439-95-4$ & Magnesium & $4.48 \mathrm{E} 3$ & ug/g dry & $6.80 \mathrm{E} 0$ & $5 / 12 / 09$ & 9E12001 & PNNL-AGG-ICP-AES \\
\hline $7439-96-5$ & Manganese & $4.30 \mathrm{E} 2$ & $\mathrm{ug} / \mathrm{g}$ dry & $2.13 \mathrm{E} 0$ & $5 / 12 / 09$ & 9E12001 & PNNL-AGG-ICP-AES \\
\hline $7439-98-7$ & Molybdenum & $<8.10 \mathrm{E} 0$ & $\mathrm{ug} / \mathrm{g}$ dry & $8.10 \mathrm{E} 0$ & $5 / 12 / 09$ & 9E12001 & PNNL-AGG-ICP-AES \\
\hline $7723-14-0$ & Phosphorus & $4.28 \mathrm{E} 2$ & $\mathrm{ug} / \mathrm{g}$ dry & $3.15 \mathrm{E} 1$ & $5 / 12 / 09$ & 9E12001 & PNNL-AGG-ICP-AES \\
\hline $7440-24-6$ & Strontium & $2.84 \mathrm{E} 1$ & ug/g dry & $1.56 \mathrm{E} 0$ & $5 / 12 / 09$ & 9E12001 & PNNL-AGG-ICP-AES \\
\hline $7440-62-2$ & Vanadium & $3.41 \mathrm{E} 1$ & ug/g dry & $4.27 \mathrm{E} 0$ & $5 / 12 / 09$ & 9E12001 & PNNL-AGG-ICP-AES \\
\hline $7440-66-6$ & Zinc & $4.28 \mathrm{E} 1$ & ug/g dry & $3.89 \mathrm{E} 0$ & $5 / 12 / 09$ & 9E12001 & PNNL-AGG-ICP-AES \\
\hline $7440-23-5$ & Sodium & $6.61 \mathrm{E} 2$ & $\mathrm{ug} / \mathrm{g}$ dry & $6.72 \mathrm{E} 1$ & $5 / 12 / 09$ & 9E12001 & PNNL-AGG-ICP-AES \\
\hline $7440-67-7$ & Zirconium & 9.92E0 & ug/g dry & $2.86 \mathrm{E} 0$ & $5 / 12 / 09$ & 9E12001 & PNNL-AGG-ICP-AES \\
\hline HEIS No. & B1YM90 & \multicolumn{3}{|c|}{ Lab ID: $\quad$ 0902002-08 } & & & \\
\hline $7429-90-5$ & Aluminum & $1.22 \mathrm{E} 4$ & ug/g dry & $2.65 \mathrm{E} 1$ & $5 / 12 / 09$ & 9E12001 & PNNL-AGG-ICP-AES \\
\hline $7440-42-8$ & Boron & $<1.14 \mathrm{E} 2$ & $\mathrm{ug} / \mathrm{g}$ dry & $1.14 \mathrm{E} 2$ & $5 / 12 / 09$ & 9E12001 & PNNL-AGG-ICP-AES \\
\hline $7440-39-3$ & Barium & $1.07 \mathrm{E} 2$ & ug/g dry & $1.61 \mathrm{E} 0$ & $5 / 12 / 09$ & 9E12001 & PNNL-AGG-ICP-AES \\
\hline $7440-41-7$ & Beryllium & $<9.05 \mathrm{E}-1$ & ug/g dry & $9.05 \mathrm{E}-1$ & $5 / 12 / 09$ & 9E12001 & PNNL-AGG-ICP-AES \\
\hline $7440-69-9$ & Bismuth & $1.14 \mathrm{E} 1$ & ug/g dry & $1.12 \mathrm{E} 1$ & $5 / 12 / 09$ & 9E12001 & PNNL-AGG-ICP-AES \\
\hline $7440-70-2$ & Calcium & $1.15 \mathrm{E} 4$ & $\mathrm{ug} / \mathrm{g}$ dry & $2.75 \mathrm{E} 1$ & $5 / 12 / 09$ & 9E12001 & PNNL-AGG-ICP-AES \\
\hline $7440-48-4$ & Cobalt & $9.12 \mathrm{E} 0$ & ug/g dry & $5.66 \mathrm{E} 0$ & $5 / 12 / 09$ & 9E12001 & PNNL-AGG-ICP-AES \\
\hline $7440-50-8$ & Copper & $1.43 \mathrm{E} 1$ & $\mathrm{ug} / \mathrm{g}$ dry & $7.57 \mathrm{E} 0$ & $5 / 12 / 09$ & 9E12001 & PNNL-AGG-ICP-AES \\
\hline $7439-89-6$ & Iron & $1.94 \mathrm{E} 4$ & $\mathrm{ug} / \mathrm{g}$ dry & $7.12 \mathrm{E} 0$ & $5 / 12 / 09$ & 9E12001 & PNNL-AGG-ICP-AES \\
\hline $7440-09-7$ & Potassium & $2.23 \mathrm{E} 3$ & $\mathrm{ug} / \mathrm{g}$ dry & $1.01 \mathrm{E} 2$ & $5 / 12 / 09$ & 9E12001 & PNNL-AGG-ICP-AES \\
\hline $7439-93-2$ & Lithium & $<2.46 \mathrm{E} 1$ & ug/g dry & $2.46 \mathrm{E} 1$ & $5 / 12 / 09$ & 9E12001 & PNNL-AGG-ICP-AES \\
\hline $7439-95-4$ & Magnesium & $7.24 \mathrm{E} 3$ & ug/g dry & $7.54 \mathrm{E} 0$ & $5 / 12 / 09$ & 9E12001 & PNNL-AGG-ICP-AES \\
\hline $7439-96-5$ & Manganese & $3.98 \mathrm{E} 2$ & ug/g dry & $2.37 \mathrm{E} 0$ & $5 / 12 / 09$ & 9E12001 & PNNL-AGG-ICP-AES \\
\hline $7439-98-7$ & Molybdenum & $<8.99 \mathrm{E} 0$ & ug/g dry & 8.99E0 & $5 / 12 / 09$ & 9E12001 & PNNL-AGG-ICP-AES \\
\hline $7723-14-0$ & Phosphorus & $6.44 \mathrm{E} 2$ & ug/g dry & $3.50 \mathrm{E} 1$ & $5 / 12 / 09$ & 9E12001 & PNNL-AGG-ICP-AES \\
\hline $7440-24-6$ & Strontium & $3.62 \mathrm{E} 1$ & $\mathrm{ug} / \mathrm{g}$ dry & $1.73 \mathrm{E} 0$ & $5 / 12 / 09$ & 9E12001 & PNNL-AGG-ICP-AES \\
\hline $7440-62-2$ & Vanadium & $2.10 \mathrm{E} 1$ & $\mathrm{ug} / \mathrm{g}$ dry & 4.74E0 & $5 / 12 / 09$ & 9E12001 & PNNL-AGG-ICP-AES \\
\hline $7440-66-6$ & Zinc & $4.60 \mathrm{E} 1$ & ug/g dry & $4.32 \mathrm{E} 0$ & $5 / 12 / 09$ & 9E12001 & PNNL-AGG-ICP-AES \\
\hline
\end{tabular}


Total Metals by PNNL-AGG-ICP-AES/Acid Extract

\begin{tabular}{|c|c|c|c|c|c|c|c|}
\hline CAS \# & Analyte & Results & Units & EQL & Analyzed & Batch & Method \\
\hline HEIS No. & B1YM90 & \multicolumn{3}{|c|}{ Lab ID: $\quad$ 0902002-08 } & & & \\
\hline $7440-23-5$ & Sodium & $2.21 \mathrm{E} 2$ & ug/g dry & $7.46 \mathrm{E} 1$ & $5 / 12 / 09$ & 9E12001 & PNNL-AGG-ICP-AES \\
\hline $7440-67-7$ & Zirconium & $8.13 \mathrm{E} 0$ & ug/g dry & $3.17 \mathrm{E} 0$ & $5 / 12 / 09$ & 9E12001 & PNNL-AGG-ICP-AES \\
\hline HEIS No. & B1YM91 & \multicolumn{3}{|c|}{ Lab ID: $\quad$ 0902002-09 } & & & \\
\hline $7429-90-5$ & Aluminum & $6.98 \mathrm{E} 3$ & ug/g dry & $2.47 \mathrm{E} 1$ & $5 / 12 / 09$ & 9E12001 & PNNL-AGG-ICP-AES \\
\hline $7440-42-8$ & Boron & $<1.06 \mathrm{E} 2$ & $\mathrm{ug} / \mathrm{g}$ dry & $1.06 \mathrm{E} 2$ & $5 / 12 / 09$ & 9E12001 & PNNL-AGG-ICP-AES \\
\hline $7440-39-3$ & Barium & $1.02 \mathrm{E} 2$ & $\mathrm{ug} / \mathrm{g}$ dry & $1.51 \mathrm{E} 0$ & $5 / 12 / 09$ & 9E12001 & PNNL-AGG-ICP-AES \\
\hline $7440-41-7$ & Beryllium & $<8.45 \mathrm{E}-1$ & $\mathrm{ug} / \mathrm{g}$ dry & $8.45 \mathrm{E}-1$ & $5 / 12 / 09$ & 9E12001 & PNNL-AGG-ICP-AES \\
\hline $7440-69-9$ & Bismuth & $<1.04 \mathrm{E} 1$ & ug/g dry & $1.04 \mathrm{E} 1$ & $5 / 12 / 09$ & 9E12001 & PNNL-AGG-ICP-AES \\
\hline $7440-70-2$ & Calcium & $3.33 \mathrm{E} 3$ & $\mathrm{ug} / \mathrm{g}$ dry & $2.57 \mathrm{E} 1$ & $5 / 12 / 09$ & 9E12001 & PNNL-AGG-ICP-AES \\
\hline $7440-48-4$ & Cobalt & 9.73E0 & ug/g dry & $5.29 \mathrm{E} 0$ & $5 / 12 / 09$ & 9E12001 & PNNL-AGG-ICP-AES \\
\hline $7440-50-8$ & Copper & $7.54 \mathrm{E} 0$ & $\mathrm{ug} / \mathrm{g}$ dry & 7.07E0 & $5 / 12 / 09$ & 9E12001 & PNNL-AGG-ICP-AES \\
\hline $7439-89-6$ & Iron & $1.98 \mathrm{E} 4$ & ug/g dry & $6.65 \mathrm{E} 0$ & $5 / 12 / 09$ & 9E12001 & PNNL-AGG-ICP-AES \\
\hline $7440-09-7$ & Potassium & $8.79 \mathrm{E} 2$ & $\mathrm{ug} / \mathrm{g}$ dry & $9.46 \mathrm{E} 1$ & $5 / 12 / 09$ & 9E12001 & PNNL-AGG-ICP-AES \\
\hline $7439-93-2$ & Lithium & $<2.30 \mathrm{E} 1$ & $\mathrm{ug} / \mathrm{g}$ dry & $2.30 \mathrm{E} 1$ & $5 / 12 / 09$ & 9E12001 & PNNL-AGG-ICP-AES \\
\hline $7439-95-4$ & Magnesium & $4.48 \mathrm{E} 3$ & $\mathrm{ug} / \mathrm{g}$ dry & 7.04E0 & $5 / 12 / 09$ & 9E12001 & PNNL-AGG-ICP-AES \\
\hline $7439-96-5$ & Manganese & $5.00 \mathrm{E} 2$ & ug/g dry & $2.21 \mathrm{E} 0$ & $5 / 12 / 09$ & 9E12001 & PNNL-AGG-ICP-AES \\
\hline $7439-98-7$ & Molybdenum & $<8.39 \mathrm{E} 0$ & $\mathrm{ug} / \mathrm{g}$ dry & 8.39E0 & $5 / 12 / 09$ & 9E12001 & PNNL-AGG-ICP-AES \\
\hline $7723-14-0$ & Phosphorus & 4.34E2 & ug/g dry & $3.27 \mathrm{E} 1$ & $5 / 12 / 09$ & 9E12001 & PNNL-AGG-ICP-AES \\
\hline $7440-24-6$ & Strontium & $1.91 \mathrm{E} 1$ & $\mathrm{ug} / \mathrm{g}$ dry & $1.61 \mathrm{E} 0$ & $5 / 12 / 09$ & 9E12001 & PNNL-AGG-ICP-AES \\
\hline $7440-62-2$ & Vanadium & $2.29 \mathrm{E} 1$ & ug/g dry & $4.42 \mathrm{E} 0$ & $5 / 12 / 09$ & 9E12001 & PNNL-AGG-ICP-AES \\
\hline $7440-66-6$ & Zinc & $3.50 \mathrm{E} 1$ & $\mathrm{ug} / \mathrm{g}$ dry & 4.03E0 & $5 / 12 / 09$ & 9E12001 & PNNL-AGG-ICP-AES \\
\hline $7440-23-5$ & Sodium & $1.99 \mathrm{E} 2$ & $\mathrm{ug} / \mathrm{g}$ dry & $6.97 \mathrm{E} 1$ & $5 / 12 / 09$ & 9E12001 & PNNL-AGG-ICP-AES \\
\hline $7440-67-7$ & Zirconium & $8.44 \mathrm{E} 0$ & $\mathrm{ug} / \mathrm{g}$ dry & $2.96 \mathrm{E} 0$ & $5 / 12 / 09$ & 9E12001 & PNNL-AGG-ICP-AES \\
\hline HEIS No. & B1YM92 & \multicolumn{3}{|c|}{ Lab ID: $\quad$ 0902002-12 } & & & \\
\hline $7429-90-5$ & Aluminum & $6.73 \mathrm{E} 3$ & $\mathrm{ug} / \mathrm{g}$ dry & $2.43 \mathrm{E} 1$ & $5 / 12 / 09$ & 9E12001 & PNNL-AGG-ICP-AES \\
\hline $7440-42-8$ & Boron & $<1.04 \mathrm{E} 2$ & $\mathrm{ug} / \mathrm{g}$ dry & $1.04 \mathrm{E} 2$ & $5 / 12 / 09$ & 9E12001 & PNNL-AGG-ICP-AES \\
\hline $7440-39-3$ & Barium & $5.87 \mathrm{E} 1$ & $\mathrm{ug} / \mathrm{g}$ dry & $1.48 \mathrm{E} 0$ & $5 / 12 / 09$ & 9E12001 & PNNL-AGG-ICP-AES \\
\hline $7440-41-7$ & Beryllium & $<8.31 \mathrm{E}-1$ & ug/g dry & $8.31 \mathrm{E}-1$ & $5 / 12 / 09$ & 9E12001 & PNNL-AGG-ICP-AES \\
\hline $7440-69-9$ & Bismuth & $<1.03 \mathrm{E} 1$ & $\mathrm{ug} / \mathrm{g}$ dry & $1.03 \mathrm{E} 1$ & $5 / 12 / 09$ & 9E12001 & PNNL-AGG-ICP-AES \\
\hline $7440-70-2$ & Calcium & $5.34 \mathrm{E} 3$ & ug/g dry & $2.53 \mathrm{E} 1$ & $5 / 12 / 09$ & 9E12001 & PNNL-AGG-ICP-AES \\
\hline $7440-48-4$ & Cobalt & $8.31 \mathrm{E} 0$ & $\mathrm{ug} / \mathrm{g}$ dry & $5.20 \mathrm{E} 0$ & $5 / 12 / 09$ & 9E12001 & PNNL-AGG-ICP-AES \\
\hline $7440-50-8$ & Copper & 7.95E0 & ug/g dry & $6.96 \mathrm{E} 0$ & $5 / 12 / 09$ & 9E12001 & PNNL-AGG-ICP-AES \\
\hline $7439-89-6$ & Iron & $1.81 \mathrm{E} 4$ & $\mathrm{ug} / \mathrm{g}$ dry & $6.54 \mathrm{E} 0$ & $5 / 12 / 09$ & 9E12001 & PNNL-AGG-ICP-AES \\
\hline $7440-09-7$ & Potassium & $6.28 \mathrm{E} 2$ & $\mathrm{ug} / \mathrm{g}$ dry & $9.31 \mathrm{E} 1$ & $5 / 12 / 09$ & 9E12001 & PNNL-AGG-ICP-AES \\
\hline $7439-93-2$ & Lithium & $<2.26 \mathrm{E} 1$ & $\mathrm{ug} / \mathrm{g}$ dry & $2.26 \mathrm{E} 1$ & $5 / 12 / 09$ & 9E12001 & PNNL-AGG-ICP-AES \\
\hline $7439-95-4$ & Magnesium & $3.93 \mathrm{E} 3$ & $\mathrm{ug} / \mathrm{g}$ dry & $6.93 \mathrm{E} 0$ & $5 / 12 / 09$ & 9E12001 & PNNL-AGG-ICP-AES \\
\hline $7439-96-5$ & Manganese & $4.57 \mathrm{E} 2$ & $\mathrm{ug} / \mathrm{g}$ dry & $2.17 \mathrm{E} 0$ & $5 / 12 / 09$ & 9E12001 & PNNL-AGG-ICP-AES \\
\hline $7439-98-7$ & Molybdenum & $<8.26 \mathrm{E} 0$ & $\mathrm{ug} / \mathrm{g}$ dry & $8.26 \mathrm{E} 0$ & $5 / 12 / 09$ & 9E12001 & PNNL-AGG-ICP-AES \\
\hline $7723-14-0$ & Phosphorus & $3.75 \mathrm{E} 2$ & $\mathrm{ug} / \mathrm{g}$ dry & $3.21 \mathrm{E} 1$ & $5 / 12 / 09$ & 9E12001 & PNNL-AGG-ICP-AES \\
\hline $7440-24-6$ & Strontium & $1.84 \mathrm{E} 1$ & ug/g dry & $1.59 \mathrm{E} 0$ & $5 / 12 / 09$ & 9E12001 & PNNL-AGG-ICP-AES \\
\hline $7440-62-2$ & Vanadium & $2.67 \mathrm{E} 1$ & $\mathrm{ug} / \mathrm{g}$ dry & $4.35 \mathrm{E} 0$ & $5 / 12 / 09$ & 9E12001 & PNNL-AGG-ICP-AES \\
\hline $7440-66-6$ & Zinc & $3.17 \mathrm{E} 1$ & ug/g dry & 3.97E0 & $5 / 12 / 09$ & 9E12001 & PNNL-AGG-ICP-AES \\
\hline $7440-23-5$ & Sodium & $2.76 \mathrm{E} 2$ & ug/g dry & $6.85 \mathrm{E} 1$ & $5 / 12 / 09$ & 9E12001 & PNNL-AGG-ICP-AES \\
\hline $7440-67-7$ & Zirconium & 7.61E0 & ug/g dry & $2.91 \mathrm{E} 0$ & $5 / 12 / 09$ & 9E12001 & PNNL-AGG-ICP-AES \\
\hline
\end{tabular}


RCRA Metals By PNNL-AGG-415/Water Extract

\begin{tabular}{|c|c|c|c|c|c|c|c|}
\hline CAS \# & Analyte & Results & Units & EQL & Analyzed & Batch & Method \\
\hline HEIS No. & B1YD07 & \multicolumn{3}{|c|}{ Lab ID: $\quad$ 0902002-01 } & & & \\
\hline $14092-98-9$ & Chromium & $<3.72 \mathrm{E}-3$ & $u g / g$ dry & $3.72 \mathrm{E}-3$ & $5 / 07 / 09$ & 9E07001 & PNNL-AGG-415 \\
\hline $7440-38-2$ & Arsenic & $1.32 \mathrm{E}-2$ & ug/g dry & $3.06 \mathrm{E}-3$ & $5 / 07 / 09$ & 9E07001 & PNNL-AGG-415 \\
\hline $14687-58-2$ & Selenium & $<8.55 \mathrm{E}-3$ & ug/g dry & $8.55 \mathrm{E}-3$ & $5 / 07 / 09$ & 9E07001 & PNNL-AGG-415 \\
\hline $14378-37-1$ & Silver & $<3.38 \mathrm{E}-3$ & ug/g dry & $3.38 \mathrm{E}-3$ & $5 / 07 / 09$ & 9E07001 & PNNL-AGG-415 \\
\hline $14336-64-2$ & Cadmium & $<6.19 \mathrm{E}-4$ & ug/g dry & $6.19 \mathrm{E}-4$ & $5 / 07 / 09$ & 9E07001 & PNNL-AGG-415 \\
\hline $14265-72-6$ & Antimony & $<7.11 \mathrm{E}-4$ & ug/g dry & 7.11E-4 & $5 / 07 / 09$ & 9E07001 & PNNL-AGG-415 \\
\hline $13966-28-4$ & Lead & $<1.26 \mathrm{E}-3$ & ug/g dry & $1.26 \mathrm{E}-3$ & $5 / 07 / 09$ & 9E07001 & PNNL-AGG-415 \\
\hline HEIS No. & B1YD08 & \multicolumn{3}{|c|}{ Lab ID: $\quad 0902002-02$} & & & \\
\hline $14092-98-9$ & Chromium & $<3.79 \mathrm{E}-3$ & ug/g dry & $3.79 \mathrm{E}-3$ & $5 / 07 / 09$ & 9E07002 & PNNL-AGG-415 \\
\hline $7440-38-2$ & Arsenic & $<3.12 \mathrm{E}-3$ & ug/g dry & $3.12 \mathrm{E}-3$ & $5 / 07 / 09$ & 9E07002 & PNNL-AGG-415 \\
\hline $14687-58-2$ & Selenium & $<8.72 \mathrm{E}-3$ & ug/g dry & $8.72 \mathrm{E}-3$ & $5 / 07 / 09$ & 9E07002 & PNNL-AGG-415 \\
\hline $14378-37-1$ & Silver & $<3.44 \mathrm{E}-3$ & ug/g dry & $3.44 \mathrm{E}-3$ & $5 / 07 / 09$ & 9E07002 & PNNL-AGG-415 \\
\hline $14336-64-2$ & Cadmium & $<6.31 \mathrm{E}-4$ & ug/g dry & $6.31 \mathrm{E}-4$ & $5 / 07 / 09$ & 9E07002 & PNNL-AGG-415 \\
\hline $14265-72-6$ & Antimony & $1.84 \mathrm{E}-3$ & ug/g dry & $7.25 \mathrm{E}-4$ & $5 / 07 / 09$ & 9E07002 & PNNL-AGG-415 \\
\hline $13966-28-4$ & Lead & $<1.28 \mathrm{E}-3$ & ug/g dry & $1.28 \mathrm{E}-3$ & $5 / 07 / 09$ & 9E07002 & PNNL-AGG-415 \\
\hline HEIS No. & B1YD09 & \multicolumn{3}{|c|}{ Lab ID: $\quad 0902002-03$} & & & \\
\hline $14092-98-9$ & Chromium & $<3.82 \mathrm{E}-3$ & ug/g dry & $3.82 \mathrm{E}-3$ & $5 / 07 / 09$ & 9E07002 & PNNL-AGG-415 \\
\hline $7440-38-2$ & Arsenic & $<3.15 \mathrm{E}-3$ & ug/g dry & $3.15 \mathrm{E}-3$ & $5 / 07 / 09$ & 9E07002 & PNNL-AGG-415 \\
\hline $14687-58-2$ & Selenium & $<8.78 \mathrm{E}-3$ & ug/g dry & $8.78 \mathrm{E}-3$ & $5 / 07 / 09$ & 9E07002 & PNNL-AGG-415 \\
\hline $14378-37-1$ & Silver & $<3.47 \mathrm{E}-3$ & ug/g dry & $3.47 \mathrm{E}-3$ & $5 / 07 / 09$ & 9E07002 & PNNL-AGG-415 \\
\hline $14336-64-2$ & Cadmium & $<6.36 \mathrm{E}-4$ & ug/g dry & $6.36 \mathrm{E}-4$ & $5 / 07 / 09$ & 9E07002 & PNNL-AGG-415 \\
\hline $14265-72-6$ & Antimony & $1.47 \mathrm{E}-3$ & ug/g dry & $7.30 \mathrm{E}-4$ & $5 / 07 / 09$ & 9E07002 & PNNL-AGG-415 \\
\hline $13966-28-4$ & Lead & $<1.29 \mathrm{E}-3$ & ug/g dry & $1.29 \mathrm{E}-3$ & $5 / 07 / 09$ & 9E07002 & PNNL-AGG-415 \\
\hline HEIS No. & B1YND4 & \multicolumn{3}{|c|}{ Lab ID: $\quad 0902002-05$} & & & \\
\hline $14092-98-9$ & Chromium & $<3.45 \mathrm{E}-3$ & ug/g dry & $3.45 \mathrm{E}-3$ & $5 / 07 / 09$ & 9E07003 & PNNL-AGG-415 \\
\hline $7440-38-2$ & Arsenic & $1.34 \mathrm{E}-2$ & ug/g dry & $2.84 \mathrm{E}-3$ & $5 / 07 / 09$ & 9E07003 & PNNL-AGG-415 \\
\hline $14687-58-2$ & Selenium & $<7.93 \mathrm{E}-3$ & ug/g dry & $7.93 \mathrm{E}-3$ & $5 / 07 / 09$ & 9E07003 & PNNL-AGG-415 \\
\hline $14378-37-1$ & Silver & $<3.13 \mathrm{E}-3$ & ug/g dry & $3.13 \mathrm{E}-3$ & $5 / 07 / 09$ & 9E07003 & PNNL-AGG-415 \\
\hline $14336-64-2$ & Cadmium & $<5.74 \mathrm{E}-4$ & ug/g dry & $5.74 \mathrm{E}-4$ & $5 / 07 / 09$ & 9E07003 & PNNL-AGG-415 \\
\hline $14265-72-6$ & Antimony & $<6.59 \mathrm{E}-4$ & ug/g dry & $6.59 \mathrm{E}-4$ & $5 / 07 / 09$ & 9E07003 & PNNL-AGG-415 \\
\hline $13966-28-4$ & Lead & $<1.16 \mathrm{E}-3$ & ug/g dry & $1.16 \mathrm{E}-3$ & $5 / 07 / 09$ & 9E07003 & PNNL-AGG-415 \\
\hline HEIS No. & B1YND5 & \multicolumn{3}{|c|}{ Lab ID: $\quad 0902002-06$} & & & \\
\hline $14092-98-9$ & Chromium & $<3.45 \mathrm{E}-3$ & ug/g dry & $3.45 \mathrm{E}-3$ & $5 / 07 / 09$ & 9E07003 & PNNL-AGG-415 \\
\hline $7440-38-2$ & Arsenic & $3.79 \mathrm{E}-3$ & ug/g dry & $2.84 \mathrm{E}-3$ & $5 / 07 / 09$ & 9E07003 & PNNL-AGG-415 \\
\hline $14687-58-2$ & Selenium & $<7.92 \mathrm{E}-3$ & ug/g dry & $7.92 \mathrm{E}-3$ & $5 / 07 / 09$ & 9E07003 & PNNL-AGG-415 \\
\hline $14378-37-1$ & Silver & $<3.13 \mathrm{E}-3$ & ug/g dry & $3.13 \mathrm{E}-3$ & $5 / 07 / 09$ & 9E07003 & PNNL-AGG-415 \\
\hline $14336-64-2$ & Cadmium & $<5.73 \mathrm{E}-4$ & ug/g dry & $5.73 \mathrm{E}-4$ & $5 / 07 / 09$ & 9E07003 & PNNL-AGG-415 \\
\hline $14265-72-6$ & Antimony & 7.66E-4 & ug/g dry & $6.59 \mathrm{E}-4$ & $5 / 07 / 09$ & 9E07003 & PNNL-AGG-415 \\
\hline $13966-28-4$ & Lead & $<1.16 \mathrm{E}-3$ & ug/g dry & $1.16 \mathrm{E}-3$ & $5 / 07 / 09$ & 9E07003 & PNNL-AGG-415 \\
\hline HEIS No. & B1YND7 & \multicolumn{3}{|c|}{ Lab ID: $\quad$ 0902002-07 } & & & \\
\hline $14092-98-9$ & Chromium & $<3.43 \mathrm{E}-3$ & ug/g dry & $3.43 \mathrm{E}-3$ & $5 / 07 / 09$ & 9Е07003 & PNNL-AGG-415 \\
\hline $7440-38-2$ & Arsenic & $4.72 \mathrm{E}-3$ & ug/g dry & $2.83 \mathrm{E}-3$ & $5 / 07 / 09$ & 9E07003 & PNNL-AGG-415 \\
\hline $14687-58-2$ & Selenium & $<7.89 \mathrm{E}-3$ & ug/g dry & $7.89 \mathrm{E}-3$ & $5 / 07 / 09$ & 9E07003 & PNNL-AGG-415 \\
\hline $14378-37-1$ & Silver & $<3.12 \mathrm{E}-3$ & ug/g dry & $3.12 \mathrm{E}-3$ & $5 / 07 / 09$ & 9E07003 & PNNL-AGG-415 \\
\hline $14336-64-2$ & Cadmium & $<5.71 \mathrm{E}-4$ & ug/g dry & $5.71 \mathrm{E}-4$ & $5 / 07 / 09$ & 9E07003 & PNNL-AGG-415 \\
\hline $14265-72-6$ & Antimony & $5.52 \mathrm{E}-3$ & ug/g dry & $6.56 \mathrm{E}-4$ & $5 / 07 / 09$ & 9E07003 & PNNL-AGG-415 \\
\hline $13966-28-4$ & Lead & $<1.16 \mathrm{E}-3$ & ug/g dry & $1.16 \mathrm{E}-3$ & $5 / 07 / 09$ & 9E07003 & PNNL-AGG-415 \\
\hline HEIS No. & B1YM90 & \multicolumn{2}{|c|}{ Lab ID: } & 2002-08 & & & \\
\hline
\end{tabular}


RCRA Metals By PNNL-AGG-415/Water Extract

\begin{tabular}{|c|c|c|c|c|c|c|c|}
\hline CAS \# & Analyte & Results & Units & EQL & Analyzed & Batch & Method \\
\hline HEIS No. & B1YM90 & \multicolumn{3}{|c|}{ Lab ID: $\quad 0902002-08$} & & & \\
\hline $14092-98-9$ & Chromium & $1.27 \mathrm{E}-1$ & $u g / g$ dry & $3.45 \mathrm{E}-3$ & $5 / 07 / 09$ & 9E07003 & PNNL-AGG-415 \\
\hline $7440-38-2$ & Arsenic & $7.51 \mathrm{E}-3$ & ug/g dry & $2.84 \mathrm{E}-3$ & $5 / 07 / 09$ & 9E07003 & PNNL-AGG-415 \\
\hline $14687-58-2$ & Selenium & $<7.92 \mathrm{E}-3$ & ug/g dry & $7.92 \mathrm{E}-3$ & $5 / 07 / 09$ & 9E07003 & PNNL-AGG-415 \\
\hline $14378-37-1$ & Silver & $<3.13 \mathrm{E}-3$ & ug/g dry & $3.13 \mathrm{E}-3$ & $5 / 07 / 09$ & 9E07003 & PNNL-AGG-415 \\
\hline $14336-64-2$ & Cadmium & $<5.73 \mathrm{E}-4$ & ug/g dry & $5.73 \mathrm{E}-4$ & $5 / 07 / 09$ & 9E07003 & PNNL-AGG-415 \\
\hline $14265-72-6$ & Antimony & $<6.59 \mathrm{E}-4$ & ug/g dry & $6.59 \mathrm{E}-4$ & $5 / 07 / 09$ & 9E07003 & PNNL-AGG-415 \\
\hline $13966-28-4$ & Lead & $<1.16 \mathrm{E}-3$ & ug/g dry & $1.16 \mathrm{E}-3$ & $5 / 07 / 09$ & 9E07003 & PNNL-AGG-415 \\
\hline HEIS No. & B1YM91 & \multicolumn{3}{|c|}{ Lab ID: $\quad 0902002-09$} & & & \\
\hline $14092-98-9$ & Chromium & $<3.69 \mathrm{E}-3$ & ug/g dry & $3.69 \mathrm{E}-3$ & $5 / 07 / 09$ & 9E07003 & PNNL-AGG-415 \\
\hline $7440-38-2$ & Arsenic & $<3.04 \mathrm{E}-3$ & ug/g dry & $3.04 \mathrm{E}-3$ & $5 / 07 / 09$ & 9E07003 & PNNL-AGG-415 \\
\hline $14687-58-2$ & Selenium & $<8.49 \mathrm{E}-3$ & ug/g dry & $8.49 \mathrm{E}-3$ & $5 / 07 / 09$ & 9E07003 & PNNL-AGG-415 \\
\hline $14378-37-1$ & Silver & $<3.35 \mathrm{E}-3$ & ug/g dry & $3.35 \mathrm{E}-3$ & $5 / 07 / 09$ & 9E07003 & PNNL-AGG-415 \\
\hline $14336-64-2$ & Cadmium & $<6.15 \mathrm{E}-4$ & ug/g dry & $6.15 \mathrm{E}-4$ & $5 / 07 / 09$ & 9E07003 & PNNL-AGG-415 \\
\hline $14265-72-6$ & Antimony & $<7.06 \mathrm{E}-4$ & ug/g dry & $7.06 \mathrm{E}-4$ & $5 / 07 / 09$ & 9E07003 & PNNL-AGG-415 \\
\hline $13966-28-4$ & Lead & $<1.25 \mathrm{E}-3$ & ug/g dry & $1.25 \mathrm{E}-3$ & $5 / 07 / 09$ & 9E07003 & PNNL-AGG-415 \\
\hline HEIS No. & B1YM92 & \multicolumn{3}{|c|}{ Lab ID: $\quad 0902002-12$} & & & \\
\hline $14092-98-9$ & Chromium & $<3.47 \mathrm{E}-3$ & ug/g dry & $3.47 \mathrm{E}-3$ & $5 / 07 / 09$ & 9E07004 & PNNL-AGG-415 \\
\hline $7440-38-2$ & Arsenic & $<2.85 \mathrm{E}-3$ & ug/g dry & $2.85 \mathrm{E}-3$ & $5 / 07 / 09$ & 9E07004 & PNNL-AGG-415 \\
\hline $14687-58-2$ & Selenium & $<7.97 \mathrm{E}-3$ & ug/g dry & $7.97 \mathrm{E}-3$ & $5 / 07 / 09$ & 9E07004 & PNNL-AGG-415 \\
\hline $14378-37-1$ & Silver & $<3.15 \mathrm{E}-3$ & ug/g dry & $3.15 \mathrm{E}-3$ & $5 / 07 / 09$ & 9E07004 & PNNL-AGG-415 \\
\hline $14336-64-2$ & Cadmium & $<5.77 \mathrm{E}-4$ & ug/g dry & $5.77 \mathrm{E}-4$ & $5 / 07 / 09$ & 9E07004 & PNNL-AGG-415 \\
\hline $14265-72-6$ & Antimony & $<6.63 \mathrm{E}-4$ & ug/g dry & $6.63 \mathrm{E}-4$ & $5 / 07 / 09$ & 9E07004 & PNNL-AGG-415 \\
\hline $13966-28-4$ & Lead & $<1.17 \mathrm{E}-3$ & ug/g dry & $1.17 \mathrm{E}-3$ & $5 / 07 / 09$ & 9E07004 & PNNL-AGG-415 \\
\hline
\end{tabular}


RCRA Metals By PNNL-AGG-415/Acid Extract

\begin{tabular}{|c|c|c|c|c|c|c|c|}
\hline CAS \# & Analyte & Results & Units & EQL & Analyzed & Batch & Method \\
\hline HEIS No. & B1YD07 & \multicolumn{3}{|c|}{ Lab ID: $\quad$ 0902002-01 } & & & \\
\hline $14092-98-9$ & Chromium & $1.43 \mathrm{E} 1$ & $u g / g$ dry & $6.46 \mathrm{E}-1$ & $5 / 08 / 09$ & 9E07006 & PNNL-AGG-415 \\
\hline $7440-38-2$ & Arsenic & $5.25 \mathrm{E}-1$ & ug/g dry & $2.81 \mathrm{E}-1$ & $5 / 08 / 09$ & 9E07006 & PNNL-AGG-415 \\
\hline $14687-58-2$ & Selenium & $<5.43 \mathrm{E}-1$ & ug/g dry & $5.43 \mathrm{E}-1$ & $5 / 08 / 09$ & 9E07006 & PNNL-AGG-415 \\
\hline $14378-37-1$ & Silver & $<1.76 \mathrm{E}-2$ & ug/g dry & $1.76 \mathrm{E}-2$ & $5 / 08 / 09$ & 9E07006 & PNNL-AGG-415 \\
\hline $14336-64-2$ & Cadmium & $<4.64 \mathrm{E}-2$ & ug/g dry & 4.64E-2 & $5 / 08 / 09$ & 9E07006 & PNNL-AGG-415 \\
\hline $14265-72-6$ & Antimony & $<1.80 \mathrm{E}-1$ & ug/g dry & $1.80 \mathrm{E}-1$ & $5 / 08 / 09$ & 9E07006 & PNNL-AGG-415 \\
\hline $13966-28-4$ & Lead & $2.14 \mathrm{E} 0$ & ug/g dry & $1.76 \mathrm{E}-1$ & $5 / 08 / 09$ & 9E07006 & PNNL-AGG-415 \\
\hline HEIS No. & B1YD08 & \multicolumn{3}{|c|}{ Lab ID: $\quad 0902002-02$} & & & \\
\hline $14092-98-9$ & Chromium & $1.36 \mathrm{E} 1$ & ug/g dry & $6.69 \mathrm{E}-1$ & $5 / 08 / 09$ & 9Е07006 & PNNL-AGG-415 \\
\hline $7440-38-2$ & Arsenic & $3.04 \mathrm{E} 0$ & ug/g dry & 2.91E-1 & $5 / 08 / 09$ & 9E07006 & PNNL-AGG-415 \\
\hline $14687-58-2$ & Selenium & $<5.63 \mathrm{E}-1$ & ug/g dry & $5.63 \mathrm{E}-1$ & $5 / 08 / 09$ & 9E07006 & PNNL-AGG-415 \\
\hline $14378-37-1$ & Silver & $4.29 \mathrm{E}-2$ & ug/g dry & $1.82 \mathrm{E}-2$ & $5 / 08 / 09$ & 9E07006 & PNNL-AGG-415 \\
\hline $14336-64-2$ & Cadmium & $6.28 \mathrm{E}-2$ & ug/g dry & 4.81E-2 & $5 / 08 / 09$ & 9E07006 & PNNL-AGG-415 \\
\hline $14265-72-6$ & Antimony & $<1.87 \mathrm{E}-1$ & ug/g dry & $1.87 \mathrm{E}-1$ & $5 / 08 / 09$ & 9E07006 & PNNL-AGG-415 \\
\hline $13966-28-4$ & Lead & $3.75 \mathrm{E} 0$ & ug/g dry & $1.82 \mathrm{E}-1$ & $5 / 08 / 09$ & 9E07006 & PNNL-AGG-415 \\
\hline HEIS No. & B1YD09 & \multicolumn{3}{|c|}{ Lab ID: $\quad 0902002-03$} & & & \\
\hline $14092-98-9$ & Chromium & $1.09 \mathrm{E} 1$ & ug/g dry & $6.64 \mathrm{E}-1$ & $5 / 08 / 09$ & 9E07006 & PNNL-AGG-415 \\
\hline $7440-38-2$ & Arsenic & $2.94 \mathrm{E}-1$ & ug/g dry & $2.89 \mathrm{E}-1$ & $5 / 08 / 09$ & 9E07006 & PNNL-AGG-415 \\
\hline $14687-58-2$ & Selenium & $<5.59 \mathrm{E}-1$ & ug/g dry & $5.59 \mathrm{E}-1$ & $5 / 08 / 09$ & 9E07006 & PNNL-AGG-415 \\
\hline $14378-37-1$ & Silver & $<1.81 \mathrm{E}-2$ & ug/g dry & $1.81 \mathrm{E}-2$ & $5 / 08 / 09$ & 9E07006 & PNNL-AGG-415 \\
\hline $14336-64-2$ & Cadmium & $<4.77 \mathrm{E}-2$ & ug/g dry & 4.77E-2 & $5 / 08 / 09$ & 9E07006 & PNNL-AGG-415 \\
\hline $14265-72-6$ & Antimony & $<1.85 \mathrm{E}-1$ & ug/g dry & $1.85 \mathrm{E}-1$ & $5 / 08 / 09$ & 9E07006 & PNNL-AGG-415 \\
\hline $13966-28-4$ & Lead & $1.08 \mathrm{E} 0$ & ug/g dry & $1.81 \mathrm{E}-1$ & $5 / 08 / 09$ & 9E07006 & PNNL-AGG-415 \\
\hline HEIS No. & B1YND4 & \multicolumn{3}{|c|}{ Lab ID: $\quad$ 0902002-05 } & & & \\
\hline $14092-98-9$ & Chromium & $1.31 \mathrm{E} 1$ & ug/g dry & $6.39 \mathrm{E}-1$ & $5 / 08 / 09$ & 9E07006 & PNNL-AGG-415 \\
\hline $7440-38-2$ & Arsenic & $3.43 \mathrm{E} 0$ & ug/g dry & $2.78 \mathrm{E}-1$ & $5 / 08 / 09$ & 9E07006 & PNNL-AGG-415 \\
\hline $14687-58-2$ & Selenium & $<5.38 \mathrm{E}-1$ & ug/g dry & $5.38 \mathrm{E}-1$ & $5 / 08 / 09$ & 9E07006 & PNNL-AGG-415 \\
\hline $14378-37-1$ & Silver & $2.86 \mathrm{E}-2$ & ug/g dry & $1.74 \mathrm{E}-2$ & $5 / 08 / 09$ & 9E07006 & PNNL-AGG-415 \\
\hline $14336-64-2$ & Cadmium & $<4.60 \mathrm{E}-2$ & ug/g dry & $4.60 \mathrm{E}-2$ & $5 / 08 / 09$ & 9E07006 & PNNL-AGG-415 \\
\hline $14265-72-6$ & Antimony & $<1.79 \mathrm{E}-1$ & ug/g dry & $1.79 \mathrm{E}-1$ & $5 / 08 / 09$ & 9E07006 & PNNL-AGG-415 \\
\hline $13966-28-4$ & Lead & $3.06 \mathrm{E} 0$ & ug/g dry & $1.74 \mathrm{E}-1$ & $5 / 08 / 09$ & 9E07006 & PNNL-AGG-415 \\
\hline HEIS No. & B1YND5 & \multicolumn{3}{|c|}{ Lab ID: $\quad 0902002-06$} & & & \\
\hline $14092-98-9$ & Chromium & $1.70 \mathrm{E} 1$ & ug/g dry & $6.77 \mathrm{E}-1$ & $5 / 08 / 09$ & 9E07006 & PNNL-AGG-415 \\
\hline $7440-38-2$ & Arsenic & $1.47 \mathrm{E} 0$ & ug/g dry & $2.95 \mathrm{E}-1$ & $5 / 08 / 09$ & 9E07006 & PNNL-AGG-415 \\
\hline $14687-58-2$ & Selenium & $<5.70 \mathrm{E}-1$ & ug/g dry & $5.70 \mathrm{E}-1$ & $5 / 08 / 09$ & 9E07006 & PNNL-AGG-415 \\
\hline $14378-37-1$ & Silver & $2.02 \mathrm{E}-2$ & ug/g dry & $1.84 \mathrm{E}-2$ & $5 / 08 / 09$ & 9E07006 & PNNL-AGG-415 \\
\hline $14336-64-2$ & Cadmium & $6.56 \mathrm{E}-2$ & ug/g dry & $4.87 \mathrm{E}-2$ & $5 / 08 / 09$ & 9E07006 & PNNL-AGG-415 \\
\hline $14265-72-6$ & Antimony & $<1.89 \mathrm{E}-1$ & ug/g dry & $1.89 \mathrm{E}-1$ & $5 / 08 / 09$ & 9E07006 & PNNL-AGG-415 \\
\hline $13966-28-4$ & Lead & 2.63E0 & ug/g dry & $1.85 \mathrm{E}-1$ & $5 / 08 / 09$ & 9E07006 & PNNL-AGG-415 \\
\hline HEIS No. & B1YND7 & \multicolumn{3}{|c|}{ Lab ID: $\quad$ 0902002-07 } & & & \\
\hline $14092-98-9$ & Chromium & $2.91 \mathrm{E} 1$ & ug/g dry & $6.65 \mathrm{E}-1$ & $5 / 08 / 09$ & 9E07006 & PNNL-AGG-415 \\
\hline $7440-38-2$ & Arsenic & $3.84 \mathrm{E}-1$ & ug/g dry & $2.90 \mathrm{E}-1$ & $5 / 08 / 09$ & 9E07006 & PNNL-AGG-415 \\
\hline $14687-58-2$ & Selenium & $<5.60 \mathrm{E}-1$ & ug/g dry & $5.60 \mathrm{E}-1$ & $5 / 08 / 09$ & 9E07006 & PNNL-AGG-415 \\
\hline $14378-37-1$ & Silver & $2.42 \mathrm{E}-2$ & ug/g dry & $1.81 \mathrm{E}-2$ & $5 / 08 / 09$ & 9E07006 & PNNL-AGG-415 \\
\hline $14336-64-2$ & Cadmium & $<4.78 \mathrm{E}-2$ & ug/g dry & $4.78 \mathrm{E}-2$ & $5 / 08 / 09$ & 9E07006 & PNNL-AGG-415 \\
\hline $14265-72-6$ & Antimony & $<1.86 \mathrm{E}-1$ & ug/g dry & $1.86 \mathrm{E}-1$ & $5 / 08 / 09$ & 9E07006 & PNNL-AGG-415 \\
\hline $13966-28-4$ & Lead & 2.67E0 & ug/g dry & $1.81 \mathrm{E}-1$ & $5 / 08 / 09$ & 9E07006 & PNNL-AGG-415 \\
\hline HEIS No. & B1YM90 & \multicolumn{2}{|c|}{ Lab ID: } & 2002-08 & & & \\
\hline
\end{tabular}


RCRA Metals By PNNL-AGG-415/Acid Extract

\begin{tabular}{|c|c|c|c|c|c|c|c|}
\hline CAS \# & Analyte & Results & Units & EQL & Analyzed & Batch & Method \\
\hline HEIS No. & B1YM90 & \multicolumn{3}{|c|}{ Lab ID: $\quad 0902002-08$} & & & \\
\hline $14092-98-9$ & Chromium & $1.78 \mathrm{E} 1$ & $u g / g$ dry & $7.38 \mathrm{E}-1$ & $5 / 11 / 09$ & 9Е07006 & PNNL-AGG-415 \\
\hline $7440-38-2$ & Arsenic & $5.50 \mathrm{E} 0$ & ug/g dry & $3.21 \mathrm{E}-1$ & $5 / 11 / 09$ & 9E07006 & PNNL-AGG-415 \\
\hline $14687-58-2$ & Selenium & $<6.21 \mathrm{E}-1$ & ug/g dry & $6.21 \mathrm{E}-1$ & $5 / 11 / 09$ & 9E07006 & PNNL-AGG-415 \\
\hline $14378-37-1$ & Silver & $6.32 \mathrm{E}-2$ & ug/g dry & $2.01 \mathrm{E}-2$ & $5 / 11 / 09$ & 9E07006 & PNNL-AGG-415 \\
\hline $14336-64-2$ & Cadmium & $6.69 \mathrm{E}-2$ & ug/g dry & $5.30 \mathrm{E}-2$ & $5 / 11 / 09$ & 9E07006 & PNNL-AGG-415 \\
\hline $14265-72-6$ & Antimony & $<2.06 \mathrm{E}-1$ & ug/g dry & $2.06 \mathrm{E}-1$ & $5 / 11 / 09$ & 9E07006 & PNNL-AGG-415 \\
\hline $13966-28-4$ & Lead & $6.02 \mathrm{E} 0$ & ug/g dry & $2.01 \mathrm{E}-1$ & $5 / 11 / 09$ & 9E07006 & PNNL-AGG-415 \\
\hline HEIS No. & B1YM91 & \multicolumn{3}{|c|}{ Lab ID: $\quad 0902002-09$} & & & \\
\hline $14092-98-9$ & Chromium & $1.72 \mathrm{E} 1$ & ug/g dry & $6.89 \mathrm{E}-1$ & $5 / 08 / 09$ & 9E07006 & PNNL-AGG-415 \\
\hline $7440-38-2$ & Arsenic & 4.16E-1 & ug/g dry & $3.00 \mathrm{E}-1$ & $5 / 08 / 09$ & 9E07006 & PNNL-AGG-415 \\
\hline $14687-58-2$ & Selenium & $<5.80 \mathrm{E}-1$ & ug/g dry & $5.80 \mathrm{E}-1$ & $5 / 08 / 09$ & 9E07006 & PNNL-AGG-415 \\
\hline $14378-37-1$ & Silver & $<1.88 \mathrm{E}-2$ & ug/g dry & $1.88 \mathrm{E}-2$ & $5 / 08 / 09$ & 9E07006 & PNNL-AGG-415 \\
\hline $14336-64-2$ & Cadmium & $<4.95 \mathrm{E}-2$ & ug/g dry & $4.95 \mathrm{E}-2$ & $5 / 08 / 09$ & 9E07006 & PNNL-AGG-415 \\
\hline $14265-72-6$ & Antimony & $<1.92 \mathrm{E}-1$ & ug/g dry & $1.92 \mathrm{E}-1$ & $5 / 08 / 09$ & 9E07006 & PNNL-AGG-415 \\
\hline $13966-28-4$ & Lead & $2.56 \mathrm{E} 0$ & ug/g dry & $1.88 \mathrm{E}-1$ & $5 / 08 / 09$ & 9E07006 & PNNL-AGG-415 \\
\hline HEIS No. & B1YM92 & \multicolumn{3}{|c|}{ Lab ID: $\quad 0902002-12$} & & & \\
\hline $14092-98-9$ & Chromium & $1.97 \mathrm{E} 1$ & ug/g dry & $6.78 \mathrm{E}-1$ & $5 / 08 / 09$ & 9E07006 & PNNL-AGG-415 \\
\hline $7440-38-2$ & Arsenic & 3.92E-1 & ug/g dry & $2.95 \mathrm{E}-1$ & $5 / 08 / 09$ & 9E07006 & PNNL-AGG-415 \\
\hline $14687-58-2$ & Selenium & $<5.71 \mathrm{E}-1$ & ug/g dry & $5.71 \mathrm{E}-1$ & $5 / 08 / 09$ & 9E07006 & PNNL-AGG-415 \\
\hline $14378-37-1$ & Silver & $<1.85 \mathrm{E}-2$ & ug/g dry & $1.85 \mathrm{E}-2$ & $5 / 08 / 09$ & 9E07006 & PNNL-AGG-415 \\
\hline $14336-64-2$ & Cadmium & $<4.87 \mathrm{E}-2$ & ug/g dry & $4.87 \mathrm{E}-2$ & $5 / 08 / 09$ & 9E07006 & PNNL-AGG-415 \\
\hline $14265-72-6$ & Antimony & $<1.89 \mathrm{E}-1$ & ug/g dry & $1.89 \mathrm{E}-1$ & $5 / 08 / 09$ & 9E07006 & PNNL-AGG-415 \\
\hline $13966-28-4$ & Lead & $2.50 \mathrm{E} 0$ & ug/g dry & $1.85 \mathrm{E}-1$ & $5 / 08 / 09$ & 9E07006 & PNNL-AGG-415 \\
\hline
\end{tabular}




\section{Carbon Analysis/Soil}

Total Organic Carbon (ug/g) by AGG-TOC-001

\begin{tabular}{|c|c|c|c|c|c|}
\hline Lab ID & HEIS No. & Results & EQL & Analyzed & Batch \\
\hline 0902002-01 & B1YD07 & $<2.00 \mathrm{E} 2$ & $2.00 \mathrm{E} 2$ & $3 / 11 / 09$ & [CALC] \\
\hline 0902002-02 & B1YD08 & $2.61 \mathrm{E} 2$ & $2.00 \mathrm{E} 2$ & $3 / 27 / 09$ & [CALC] \\
\hline 0902002-03 & B1YD09 & $2.00 \mathrm{E} 2$ & $2.00 \mathrm{E} 2$ & $3 / 27 / 09$ & [CALC] \\
\hline 0902002-04 & B1YD74 & $<2.00 \mathrm{E} 2$ & $2.00 \mathrm{E} 2$ & $3 / 27 / 09$ & [CALC] \\
\hline 0902002-05 & B1YND4 & $5.03 \mathrm{E} 2$ & $2.00 \mathrm{E} 2$ & $4 / 21 / 09$ & [CALC] \\
\hline 0902002-06 & B1YND5 & $2.68 \mathrm{E} 2$ & $2.00 \mathrm{E} 2$ & $4 / 21 / 09$ & [CALC] \\
\hline 0902002-07 & B1YND7 & $3.59 \mathrm{E} 2$ & $2.00 \mathrm{E} 2$ & $4 / 21 / 09$ & [CALC] \\
\hline 0902002-08 & B1YM90 & $3.16 \mathrm{E} 3$ & $2.00 \mathrm{E} 2$ & $4 / 21 / 09$ & [CALC] \\
\hline 0902002-09 & B1YM91 & $3.92 \mathrm{E} 2$ & $2.00 \mathrm{E} 2$ & $4 / 22 / 09$ & [CALC] \\
\hline 0902002-10 & B1YND6 & $<2.00 \mathrm{E} 2$ & $2.00 \mathrm{E} 2$ & $4 / 22 / 09$ & [CALC] \\
\hline 0902002-11 & B1YD75 & $2.46 \mathrm{E} 2$ & $2.00 \mathrm{E} 2$ & $4 / 22 / 09$ & [CALC] \\
\hline 0902002-12 & B1YM92 & $2.52 \mathrm{E} 2$ & $2.00 \mathrm{E} 2$ & $4 / 22 / 09$ & [CALC] \\
\hline 0902002-13 & B1YM93 & 8.97E2 & $2.00 \mathrm{E} 2$ & $4 / 22 / 09$ & [CALC] \\
\hline 0902002-14 & B1YNV5 & $<2.00 \mathrm{E} 2$ & $2.00 \mathrm{E} 2$ & $4 / 22 / 09$ & [CALC] \\
\hline 0902002-15 & B1YD76 & $<2.00 \mathrm{E} 2$ & $2.00 \mathrm{E} 2$ & $5 / 06 / 09$ & [CALC] \\
\hline 0902002-16 & B1YD88 & $2.85 \mathrm{E} 2$ & $2.00 \mathrm{E} 2$ & $5 / 06 / 09$ & [CALC] \\
\hline 0902002-17 & B1YD77 & $3.47 \mathrm{E} 2$ & $2.00 \mathrm{E} 2$ & $5 / 06 / 09$ & [CALC] \\
\hline 0902002-18 & B1YD78 & $2.05 \mathrm{E} 2$ & $2.00 \mathrm{E} 2$ & $5 / 06 / 09$ & [CALC] \\
\hline 0902002-19 & B1YD79 & $5.93 \mathrm{E} 2$ & $2.00 \mathrm{E} 2$ & $5 / 06 / 09$ & [CALC] \\
\hline 0902002-20 & B1YND9 & $4.81 \mathrm{E} 2$ & $2.00 \mathrm{E} 2$ & $5 / 07 / 09$ & [CALC] \\
\hline 0902002-21 & B1YNF0 & $5.76 \mathrm{E} 2$ & $2.00 \mathrm{E} 2$ & $5 / 07 / 09$ & [CALC] \\
\hline $0902002-22$ & B1YNF1 & $1.53 \mathrm{E} 3$ & $2.00 \mathrm{E} 2$ & $5 / 07 / 09$ & [CALC] \\
\hline $0902002-23$ & B1YD80 & $5.21 \mathrm{E} 2$ & $2.00 \mathrm{E} 2$ & $5 / 07 / 09$ & [CALC] \\
\hline 0902002-24 & B1YD81 & $2.22 \mathrm{E} 2$ & $2.00 \mathrm{E} 2$ & $5 / 07 / 09$ & [CALC] \\
\hline $0902002-25$ & B1YD82 & $1.99 \mathrm{E} 3$ & $2.00 \mathrm{E} 2$ & $5 / 07 / 09$ & [CALC] \\
\hline $0902002-26$ & B1YNF2 & $2.36 \mathrm{E} 2$ & $2.00 \mathrm{E} 2$ & $6 / 02 / 09$ & [CALC] \\
\hline $0902002-27$ & B1YNF3 & $3.75 \mathrm{E} 2$ & $2.00 \mathrm{E} 2$ & $6 / 02 / 09$ & [CALC] \\
\hline $0902002-28$ & B1YNF4 & $<2.00 \mathrm{E} 2$ & $2.00 \mathrm{E} 2$ & $6 / 02 / 09$ & [CALC] \\
\hline 0902002-29 & B1YNF5 & $<2.00 \mathrm{E} 2$ & $2.00 \mathrm{E} 2$ & $6 / 02 / 09$ & [CALC] \\
\hline 0902002-30 & B1YNF6 & $2.74 \mathrm{E} 2$ & $2.00 \mathrm{E} 2$ & $6 / 02 / 09$ & [CALC] \\
\hline 0902002-31 & B1YD83 & $2.26 \mathrm{E} 2$ & $2.00 \mathrm{E} 2$ & $6 / 02 / 09$ & [CALC] \\
\hline 0902002-37 & B1YM97 & $<2.00 \mathrm{E} 2$ & $2.00 \mathrm{E} 2$ & $10 / 05 / 09$ & [CALC] \\
\hline
\end{tabular}




\section{Carbon Analysis/Soil}

\begin{tabular}{|c|c|c|c|c|c|}
\hline $\begin{array}{l}\text { Total Carl } \\
\text { Lab ID }\end{array}$ & $\begin{array}{l}\text { (ug/g) by } \\
\text { HEIS No. }\end{array}$ & Results & EQL & Analyzed & Batch \\
\hline 0902002-01 & B1YD07 & $2.42 \mathrm{E} 3$ & $2.00 \mathrm{E} 2$ & $3 / 10 / 09$ & $9 \mathrm{C} 10005$ \\
\hline 0902002-02 & B1YD08 & $2.61 \mathrm{E} 2$ & $2.00 \mathrm{E} 2$ & $3 / 26 / 09$ & $9 \mathrm{C} 25003$ \\
\hline 0902002-03 & B1YD09 & $2.00 \mathrm{E} 2$ & $2.00 \mathrm{E} 2$ & $3 / 26 / 09$ & $9 \mathrm{C} 25003$ \\
\hline 0902002-04 & B1YD74 & $<2.00 \mathrm{E} 2$ & $2.00 \mathrm{E} 2$ & $3 / 26 / 09$ & $9 \mathrm{C} 25003$ \\
\hline 0902002-05 & B1YND4 & $2.54 \mathrm{E} 3$ & $2.00 \mathrm{E} 2$ & $4 / 16 / 09$ & 9D15004 \\
\hline 0902002-06 & B1YND5 & $5.31 \mathrm{E} 2$ & $2.00 \mathrm{E} 2$ & $4 / 16 / 09$ & 9D15004 \\
\hline 0902002-07 & B1YND7 & $3.59 \mathrm{E} 2$ & $2.00 \mathrm{E} 2$ & $4 / 16 / 09$ & 9D15004 \\
\hline 0902002-08 & B1YM90 & $6.70 \mathrm{E} 3$ & $2.00 \mathrm{E} 2$ & $4 / 16 / 09$ & 9D15004 \\
\hline 0902002-09 & B1YM91 & $3.92 \mathrm{E} 2$ & $2.00 \mathrm{E} 2$ & $4 / 16 / 09$ & 9D15004 \\
\hline 0902002-10 & B1YND6 & $2.62 \mathrm{E} 3$ & $2.00 \mathrm{E} 2$ & $4 / 16 / 09$ & 9D15004 \\
\hline 0902002-11 & B1YD75 & $1.08 \mathrm{E} 3$ & $2.00 \mathrm{E} 2$ & $4 / 16 / 09$ & 9D15004 \\
\hline 0902002-12 & B1YM92 & $1.16 \mathrm{E} 3$ & $2.00 \mathrm{E} 2$ & $4 / 17 / 09$ & 9D15004 \\
\hline 0902002-13 & B1YM93 & $8.97 \mathrm{E} 2$ & $2.00 \mathrm{E} 2$ & $4 / 17 / 09$ & 9D15004 \\
\hline 0902002-14 & B1YNV5 & $<2.00 \mathrm{E} 2$ & $2.00 \mathrm{E} 2$ & $4 / 17 / 09$ & 9D15004 \\
\hline 0902002-15 & B1YD76 & $2.17 \mathrm{E} 3$ & $2.00 \mathrm{E} 2$ & $5 / 05 / 09$ & 9E05001 \\
\hline 0902002-16 & B1YD88 & $3.33 \mathrm{E} 3$ & $2.00 \mathrm{E} 2$ & $5 / 05 / 09$ & 9E05001 \\
\hline 0902002-17 & B1YD77 & $5.95 \mathrm{E} 2$ & $2.00 \mathrm{E} 2$ & $5 / 06 / 09$ & 9E05001 \\
\hline 0902002-18 & B1YD78 & $2.05 \mathrm{E} 2$ & $2.00 \mathrm{E} 2$ & $5 / 06 / 09$ & 9E05001 \\
\hline 0902002-19 & B1YD79 & $5.93 \mathrm{E} 2$ & $2.00 \mathrm{E} 2$ & $5 / 06 / 09$ & 9E05001 \\
\hline 0902002-20 & B1YND9 & $1.76 \mathrm{E} 3$ & $2.00 \mathrm{E} 2$ & $5 / 06 / 09$ & 9E05001 \\
\hline 0902002-21 & B1YNF0 & $1.26 \mathrm{E} 3$ & $2.00 \mathrm{E} 2$ & $5 / 06 / 09$ & 9E05001 \\
\hline 0902002-22 & B1YNF1 & $3.13 \mathrm{E} 4$ & $2.00 \mathrm{E} 2$ & $5 / 06 / 09$ & 9E05001 \\
\hline $0902002-23$ & B1YD80 & $5.21 \mathrm{E} 2$ & $2.00 \mathrm{E} 2$ & $5 / 06 / 09$ & 9E05001 \\
\hline 0902002-24 & B1YD81 & $2.22 \mathrm{E} 2$ & $2.00 \mathrm{E} 2$ & $5 / 06 / 09$ & 9E05001 \\
\hline $0902002-25$ & B1YD82 & $1.99 \mathrm{E} 3$ & $2.00 \mathrm{E} 2$ & $5 / 06 / 09$ & 9E05001 \\
\hline 0902002-26 & B1YNF2 & $8.39 \mathrm{E} 2$ & $2.00 \mathrm{E} 2$ & $6 / 02 / 09$ & 9F02001 \\
\hline $0902002-27$ & B1YNF3 & $3.75 \mathrm{E} 2$ & $2.00 \mathrm{E} 2$ & $6 / 02 / 09$ & 9F02001 \\
\hline 0902002-28 & B1YNF4 & $<2.00 \mathrm{E} 2$ & $2.00 \mathrm{E} 2$ & $6 / 02 / 09$ & 9F02001 \\
\hline 0902002-29 & B1YNF5 & $<2.00 \mathrm{E} 2$ & $2.00 \mathrm{E} 2$ & $6 / 02 / 09$ & 9F02001 \\
\hline 0902002-30 & B1YNF6 & $7.26 \mathrm{E} 2$ & $2.00 \mathrm{E} 2$ & $6 / 02 / 09$ & 9F02001 \\
\hline 0902002-31 & B1YD83 & $2.26 \mathrm{E} 2$ & $2.00 \mathrm{E} 2$ & $6 / 02 / 09$ & 9F02001 \\
\hline 0902002-37 & B1YM97 & 7.03E2 & $2.00 \mathrm{E} 2$ & $10 / 05 / 09$ & 9J05001 \\
\hline
\end{tabular}




\begin{tabular}{|c|c|c|c|c|c|}
\hline \multicolumn{6}{|c|}{ Carbon Analysis/Soil } \\
\hline \multicolumn{6}{|c|}{ Total Inorganic Carbon (ug/g) by AGG-TOC-001 } \\
\hline Lab ID & HEIS No. & Results & EQL & Analyzed & Batch \\
\hline $0902002-01$ & B1YD07 & $2.40 \mathrm{E} 3$ & $2.00 \mathrm{E} 2$ & $3 / 11 / 09$ & 9C11001 \\
\hline $0902002-02$ & B1YD08 & $<2.00 \mathrm{E} 2$ & $2.00 \mathrm{E} 2$ & $3 / 27 / 09$ & 9C25005 \\
\hline $0902002-03$ & B1YD09 & $<2.00 \mathrm{E} 2$ & $2.00 \mathrm{E} 2$ & $3 / 27 / 09$ & 9C25005 \\
\hline 0902002-04 & B1YD74 & $<2.00 \mathrm{E} 2$ & $2.00 \mathrm{E} 2$ & $3 / 27 / 09$ & 9C25005 \\
\hline $0902002-05$ & B1YND4 & $2.04 \mathrm{E} 3$ & $2.00 \mathrm{E} 2$ & $4 / 21 / 09$ & 9D16001 \\
\hline 0902002-06 & B1YND5 & $2.63 \mathrm{E} 2$ & $2.00 \mathrm{E} 2$ & $4 / 21 / 09$ & 9D16001 \\
\hline 0902002-07 & B1YND7 & $<2.00 \mathrm{E} 2$ & $2.00 \mathrm{E} 2$ & $4 / 21 / 09$ & 9D16001 \\
\hline 0902002-08 & B1YM90 & $3.53 \mathrm{E} 3$ & $2.00 \mathrm{E} 2$ & $4 / 21 / 09$ & 9D16001 \\
\hline 0902002-09 & B1YM91 & $<2.00 \mathrm{E} 2$ & $2.00 \mathrm{E} 2$ & $4 / 22 / 09$ & 9D16001 \\
\hline 0902002-10 & B1YND6 & $2.46 \mathrm{E} 3$ & $2.00 \mathrm{E} 2$ & $4 / 22 / 09$ & 9D16001 \\
\hline 0902002-11 & B1YD75 & $8.30 \mathrm{E} 2$ & $2.00 \mathrm{E} 2$ & $4 / 22 / 09$ & 9D16001 \\
\hline 0902002-12 & B1YM92 & $9.04 \mathrm{E} 2$ & $2.00 \mathrm{E} 2$ & $4 / 22 / 09$ & 9D16001 \\
\hline $0902002-13$ & B1YM93 & $<2.00 \mathrm{E} 2$ & $2.00 \mathrm{E} 2$ & $4 / 22 / 09$ & 9D16001 \\
\hline 0902002-14 & B1YNV5 & $<2.00 \mathrm{E} 2$ & $2.00 \mathrm{E} 2$ & $4 / 22 / 09$ & 9D16001 \\
\hline 0902002-15 & B1YD76 & $2.04 \mathrm{E} 3$ & $2.00 \mathrm{E} 2$ & $5 / 06 / 09$ & 9Е06001 \\
\hline 0902002-16 & B1YD88 & $3.04 \mathrm{E} 3$ & $2.00 \mathrm{E} 2$ & $5 / 06 / 09$ & 9E06001 \\
\hline 0902002-17 & B1YD77 & $2.48 \mathrm{E} 2$ & $2.00 \mathrm{E} 2$ & $5 / 06 / 09$ & 9E06001 \\
\hline 0902002-18 & B1YD78 & $<2.00 \mathrm{E} 2$ & $2.00 \mathrm{E} 2$ & $5 / 06 / 09$ & 9E06001 \\
\hline 0902002-19 & B1YD79 & $<2.00 \mathrm{E} 2$ & $2.00 \mathrm{E} 2$ & $5 / 06 / 09$ & 9E06001 \\
\hline $0902002-20$ & B1YND9 & $1.28 \mathrm{E} 3$ & $2.00 \mathrm{E} 2$ & $5 / 07 / 09$ & 9E06001 \\
\hline 0902002-21 & B1YNF0 & $6.82 \mathrm{E} 2$ & $2.00 \mathrm{E} 2$ & $5 / 07 / 09$ & 9E06001 \\
\hline $0902002-22$ & B1YNF1 & $2.98 \mathrm{E} 4$ & $2.00 \mathrm{E} 2$ & $5 / 07 / 09$ & 9E06001 \\
\hline $0902002-23$ & B1YD80 & $<2.00 \mathrm{E} 2$ & $2.00 \mathrm{E} 2$ & $5 / 07 / 09$ & 9E06001 \\
\hline 0902002-24 & B1YD81 & $<2.00 \mathrm{E} 2$ & $2.00 \mathrm{E} 2$ & $5 / 07 / 09$ & 9E06001 \\
\hline $0902002-25$ & B1YD82 & $<2.00 \mathrm{E} 2$ & $2.00 \mathrm{E} 2$ & $5 / 07 / 09$ & 9E06001 \\
\hline $0902002-26$ & B1YNF2 & $6.02 \mathrm{E} 2$ & $2.00 \mathrm{E} 2$ & $6 / 02 / 09$ & 9F02002 \\
\hline $0902002-27$ & B1YNF3 & $<2.00 \mathrm{E} 2$ & $2.00 \mathrm{E} 2$ & $6 / 02 / 09$ & 9F02002 \\
\hline 0902002-28 & B1YNF4 & $<2.00 \mathrm{E} 2$ & $2.00 \mathrm{E} 2$ & $6 / 02 / 09$ & 9F02002 \\
\hline 0902002-29 & B1YNF5 & $<2.00 \mathrm{E} 2$ & $2.00 \mathrm{E} 2$ & $6 / 02 / 09$ & 9F02002 \\
\hline 0902002-30 & B1YNF6 & $4.52 \mathrm{E} 2$ & $2.00 \mathrm{E} 2$ & $6 / 02 / 09$ & 9F02002 \\
\hline 0902002-31 & B1YD83 & $<2.00 \mathrm{E} 2$ & $2.00 \mathrm{E} 2$ & $6 / 02 / 09$ & 9F02002 \\
\hline 0902002-37 & B1YM97 & $5.39 \mathrm{E} 2$ & $2.00 \mathrm{E} 2$ & $10 / 05 / 09$ & 9J05001 \\
\hline
\end{tabular}




\section{GEA/Soil}

\begin{tabular}{|c|c|c|c|c|c|c|c|c|}
\hline CAS \# & Analyte & Results & Units & MDA & UNC & Analyzed & Batch & Method \\
\hline HEIS No. & B1YD07 & \multicolumn{3}{|c|}{ Lab ID: $\quad$ 0902002-01 } & & & & \\
\hline $10198-40-0$ & Cobalt-60 & $<1.96 \mathrm{E}-1$ & pCi/g dry & $1.96 \mathrm{E}-1$ & & $4 / 23 / 09$ & 9D23001 & AGG-RRL-001 \\
\hline $10045-97-3$ & Cesium-137 & $<2.29 \mathrm{E}-1$ & pCi/g dry & $2.29 \mathrm{E}-1$ & & 4/23/09 & 9D23001 & AGG-RRL-001 \\
\hline $14683-23-9$ & Europium-152 & $<8.07 \mathrm{E}-1$ & pCi/g dry & $8.07 \mathrm{E}-1$ & & $4 / 23 / 09$ & 9D23001 & AGG-RRL-001 \\
\hline $15585-10-1$ & Europium-154 & $<4.85 \mathrm{E}-1$ & pCi/g dry & $4.85 \mathrm{E}-1$ & & $4 / 23 / 09$ & 9D23001 & AGG-RRL-001 \\
\hline $14391-16-3$ & Europium-155 & $<7.83 \mathrm{E}-1$ & pCi/g dry & $7.83 \mathrm{E}-1$ & & $4 / 23 / 09$ & 9D23001 & AGG-RRL-001 \\
\hline HEIS No. & B1YD08 & \multicolumn{3}{|c|}{ Lab ID: $\quad$ 0902002-02 } & & & & \\
\hline $10198-40-0$ & Cobalt- 60 & $<1.94 \mathrm{E}-1$ & pCi/g dry & $1.94 \mathrm{E}-1$ & & $4 / 23 / 09$ & 9D23001 & AGG-RRL-001 \\
\hline $10045-97-3$ & Cesium-137 & $<2.37 \mathrm{E}-1$ & pCi/g dry & $2.37 \mathrm{E}-1$ & & $4 / 23 / 09$ & 9D23001 & AGG-RRL-001 \\
\hline $14683-23-9$ & Europium-152 & $<8.93 \mathrm{E}-1$ & pCi/g dry & $8.93 \mathrm{E}-1$ & & 4/23/09 & 9D23001 & AGG-RRL-001 \\
\hline $15585-10-1$ & Europium-154 & $<5.15 \mathrm{E}-1$ & pCi/g dry & $5.15 \mathrm{E}-1$ & & $4 / 23 / 09$ & 9D23001 & AGG-RRL-001 \\
\hline $14391-16-3$ & Europium-155 & $<8.93 \mathrm{E}-1$ & pCi/g dry & $8.93 \mathrm{E}-1$ & & $4 / 23 / 09$ & 9D23001 & AGG-RRL-001 \\
\hline HEIS No. & B1YD09 & \multicolumn{3}{|c|}{ Lab ID: $\quad 0902002-03$} & & & & \\
\hline $10198-40-0$ & Cobalt-60 & $<1.64 \mathrm{E}-1$ & pCi/g dry & $1.64 \mathrm{E}-1$ & & $4 / 24 / 09$ & 9D23001 & AGG-RRL-001 \\
\hline $10045-97-3$ & Cesium-137 & $<1.92 \mathrm{E}-1$ & pCi/g dry & $1.92 \mathrm{E}-1$ & & $4 / 24 / 09$ & 9D23001 & AGG-RRL-001 \\
\hline $14683-23-9$ & Europium-152 & $<6.42 \mathrm{E}-1$ & pCi/g dry & $6.42 \mathrm{E}-1$ & & $4 / 24 / 09$ & 9D23001 & AGG-RRL-001 \\
\hline $15585-10-1$ & Europium-154 & $<4.07 \mathrm{E}-1$ & pCi/g dry & $4.07 \mathrm{E}-1$ & & $4 / 24 / 09$ & 9D23001 & AGG-RRL-001 \\
\hline $14391-16-3$ & Europium-155 & $<6.25 \mathrm{E}-1$ & pCi/g dry & $6.25 \mathrm{E}-1$ & & $4 / 24 / 09$ & 9D23001 & AGG-RRL-001 \\
\hline HEIS No. & B1YD74 & \multicolumn{3}{|c|}{ Lab ID: $\quad$ 0902002-04 } & & & & \\
\hline $10198-40-0$ & Cobalt- 60 & $<1.97 \mathrm{E}-1$ & pCi/g dry & $1.97 \mathrm{E}-1$ & & $4 / 24 / 09$ & 9D23001 & AGG-RRL-001 \\
\hline $10045-97-3$ & Cesium-137 & $<2.22 \mathrm{E}-1$ & pCi/g dry & $2.22 \mathrm{E}-1$ & & $4 / 24 / 09$ & 9D23001 & AGG-RRL-001 \\
\hline $14683-23-9$ & Europium-152 & $<8.83 \mathrm{E}-1$ & pCi/g dry & $8.83 \mathrm{E}-1$ & & $4 / 24 / 09$ & 9D23001 & AGG-RRL-001 \\
\hline $15585-10-1$ & Europium-154 & $<5.07 \mathrm{E}-1$ & pCi/g dry & $5.07 \mathrm{E}-1$ & & $4 / 24 / 09$ & 9D23001 & AGG-RRL-001 \\
\hline $14391-16-3$ & Europium-155 & $<8.66 \mathrm{E}-1$ & pCi/g dry & $8.66 \mathrm{E}-1$ & & $4 / 24 / 09$ & 9D23001 & AGG-RRL-001 \\
\hline HEIS No. & B1YND4 & \multicolumn{3}{|c|}{ Lab ID: $\quad$ 0902002-05 } & & & & \\
\hline $10198-40-0$ & Cobalt- 60 & $<1.62 \mathrm{E}-1$ & pCi/g dry & $1.62 \mathrm{E}-1$ & & $4 / 24 / 09$ & 9D23001 & AGG-RRL-001 \\
\hline $10045-97-3$ & Cesium-137 & $<2.00 \mathrm{E}-1$ & pCi/g dry & $2.00 \mathrm{E}-1$ & & $4 / 24 / 09$ & 9D23001 & AGG-RRL-001 \\
\hline $14683-23-9$ & Europium-152 & $<6.65 \mathrm{E}-1$ & pCi/g dry & $6.65 \mathrm{E}-1$ & & $4 / 24 / 09$ & 9D23001 & AGG-RRL-001 \\
\hline $15585-10-1$ & Europium-154 & $<4.22 \mathrm{E}-1$ & pCi/g dry & $4.22 \mathrm{E}-1$ & & $4 / 24 / 09$ & 9D23001 & AGG-RRL-001 \\
\hline $14391-16-3$ & Europium-155 & $<6.61 \mathrm{E}-1$ & pCi/g dry & $6.61 \mathrm{E}-1$ & & $4 / 24 / 09$ & 9D23001 & AGG-RRL-001 \\
\hline HEIS No. & B1YND5 & \multicolumn{3}{|c|}{ Lab ID: $\quad$ 0902002-06 } & & & & \\
\hline $10198-40-0$ & Cobalt- 60 & $<1.98 \mathrm{E}-1$ & pCi/g dry & $1.98 \mathrm{E}-1$ & & $4 / 24 / 09$ & 9D23001 & AGG-RRL-001 \\
\hline $10045-97-3$ & Cesium-137 & $<2.28 \mathrm{E}-1$ & pCi/g dry & $2.28 \mathrm{E}-1$ & & $4 / 24 / 09$ & 9D23001 & AGG-RRL-001 \\
\hline $14683-23-9$ & Europium-152 & $<9.14 \mathrm{E}-1$ & pCi/g dry & $9.14 \mathrm{E}-1$ & & $4 / 24 / 09$ & 9D23001 & AGG-RRL-001 \\
\hline $15585-10-1$ & Europium-154 & $<5.09 \mathrm{E}-1$ & pCi/g dry & $5.09 \mathrm{E}-1$ & & $4 / 24 / 09$ & 9D23001 & AGG-RRL-001 \\
\hline 14391-16-3 & Europium-155 & $<8.54 \mathrm{E}-1$ & pCi/g dry & $8.54 \mathrm{E}-1$ & & $4 / 24 / 09$ & 9D23001 & AGG-RRL-001 \\
\hline HEIS No. & B1YND7 & \multicolumn{3}{|c|}{ Lab ID: $\quad$ 0902002-07 } & & & & \\
\hline $10198-40-0$ & Cobalt- 60 & $<1.79 \mathrm{E}-1$ & pCi/g dry & $1.79 \mathrm{E}-1$ & & $4 / 27 / 09$ & 9D23001 & AGG-RRL-001 \\
\hline 10045-97-3 & Cesium-137 & $<2.13 \mathrm{E}-1$ & pCi/g dry & $2.13 \mathrm{E}-1$ & & $4 / 27 / 09$ & 9D23001 & AGG-RRL-001 \\
\hline $14683-23-9$ & Europium-152 & $<7.38 \mathrm{E}-1$ & pCi/g dry & $7.38 \mathrm{E}-1$ & & $4 / 27 / 09$ & 9D23001 & AGG-RRL-001 \\
\hline $15585-10-1$ & Europium-154 & $<4.68 \mathrm{E}-1$ & pCi/g dry & $4.68 \mathrm{E}-1$ & & $4 / 27 / 09$ & 9D23001 & AGG-RRL-001 \\
\hline $14391-16-3$ & Europium-155 & $<7.34 \mathrm{E}-1$ & pCi/g dry & $7.34 \mathrm{E}-1$ & & $4 / 27 / 09$ & 9D23001 & AGG-RRL-001 \\
\hline HEIS No. & B1YM90 & \multicolumn{3}{|c|}{ Lab ID: $\quad$ 0902002-08 } & & & & \\
\hline $10198-40-0$ & Cobalt- 60 & $<2.82 \mathrm{E}-1$ & pCi/g dry & $2.82 \mathrm{E}-1$ & & $4 / 27 / 09$ & 9D23001 & AGG-RRL-001 \\
\hline $10045-97-3$ & Cesium-137 & $<3.11 \mathrm{E}-1$ & pCi/g dry & $3.11 \mathrm{E}-1$ & & $4 / 27 / 09$ & 9D23001 & AGG-RRL-001 \\
\hline $14683-23-9$ & Europium-152 & $<1.11 \mathrm{E} 0$ & pCi/g dry & $1.11 \mathrm{E} 0$ & & $4 / 27 / 09$ & 9D23001 & AGG-RRL-001 \\
\hline $15585-10-1$ & Europium-154 & $<6.60 \mathrm{E}-1$ & pCi/g dry & $6.60 \mathrm{E}-1$ & & $4 / 27 / 09$ & 9D23001 & AGG-RRL-001 \\
\hline 14391-16-3 & Europium-155 & $<1.13 \mathrm{E} 0$ & pCi/g dry & $1.13 \mathrm{E} 0$ & & $4 / 27 / 09$ & 9D23001 & AGG-RRL-001 \\
\hline
\end{tabular}




\section{GEA/Soil}

\begin{tabular}{|c|c|c|c|c|c|c|c|c|}
\hline CAS \# & Analyte & Results & Units & MDA & UNC & Analyzed & Batch & Method \\
\hline HEIS No. & B1YM91 & \multicolumn{4}{|c|}{ Lab ID: $\quad$ 0902002-09 } & & & \\
\hline $10198-40-0$ & Cobalt-60 & $<1.55 \mathrm{E}-1$ & pCi/g dry & $1.55 \mathrm{E}-1$ & & $4 / 27 / 09$ & 9D23001 & AGG-RRL-001 \\
\hline $10045-97-3$ & Cesium-137 & $<2.06 \mathrm{E}-1$ & pCi/g dry & $2.06 \mathrm{E}-1$ & & $4 / 27 / 09$ & 9D23001 & AGG-RRL-001 \\
\hline $14683-23-9$ & Europium-152 & $<6.74 \mathrm{E}-1$ & pCi/g dry & $6.74 \mathrm{E}-1$ & & $4 / 27 / 09$ & 9D23001 & AGG-RRL-001 \\
\hline $15585-10-1$ & Europium-154 & $<4.27 \mathrm{E}-1$ & pCi/g dry & $4.27 \mathrm{E}-1$ & & $4 / 27 / 09$ & 9D23001 & AGG-RRL-001 \\
\hline $14391-16-3$ & Europium-155 & $<6.61 \mathrm{E}-1$ & pCi/g dry & $6.61 \mathrm{E}-1$ & & $4 / 27 / 09$ & 9D23001 & AGG-RRL-001 \\
\hline HEIS No. & B1YND6 & \multicolumn{4}{|c|}{ Lab ID: $\quad$ 0902002-10 } & & & \\
\hline $10198-40-0$ & Cobalt-60 & $<2.09 \mathrm{E}-1$ & pCi/g dry & $2.09 \mathrm{E}-1$ & & $4 / 27 / 09$ & 9D23001 & AGG-RRL-001 \\
\hline $10045-97-3$ & Cesium-137 & $<2.38 \mathrm{E}-1$ & pCi/g dry & $2.38 \mathrm{E}-1$ & & $4 / 27 / 09$ & 9D23001 & AGG-RRL-001 \\
\hline $14683-23-9$ & Europium-152 & $<9.14 \mathrm{E}-1$ & pCi/g dry & $9.14 \mathrm{E}-1$ & & $4 / 27 / 09$ & 9D23001 & AGG-RRL-001 \\
\hline $15585-10-1$ & Europium-154 & $<5.41 \mathrm{E}-1$ & pCi/g dry & $5.41 \mathrm{E}-1$ & & $4 / 27 / 09$ & 9D23001 & AGG-RRL-001 \\
\hline $14391-16-3$ & Europium-155 & $<8.97 \mathrm{E}-1$ & pCi/g dry & 8.97E-1 & & $4 / 27 / 09$ & 9D23001 & AGG-RRL-001 \\
\hline HEIS No. & B1YD75 & \multicolumn{4}{|c|}{ Lab ID: $\quad 0902002-11$} & & & \\
\hline $10198-40-0$ & Cobalt- 60 & $<1.36 \mathrm{E}-1$ & pCi/g dry & $1.36 \mathrm{E}-1$ & & $4 / 29 / 09$ & 9D23001 & AGG-RRL-001 \\
\hline $10045-97-3$ & Cesium-137 & $<1.72 \mathrm{E}-1$ & $\mathrm{pCi} / \mathrm{g}$ dry & $1.72 \mathrm{E}-1$ & & 4/29/09 & 9D23001 & AGG-RRL-001 \\
\hline $14683-23-9$ & Europium-152 & $<5.71 \mathrm{E}-1$ & pCi/g dry & $5.71 \mathrm{E}-1$ & & $4 / 29 / 09$ & 9D23001 & AGG-RRL-001 \\
\hline $15585-10-1$ & Europium-154 & $<3.54 \mathrm{E}-1$ & pCi/g dry & $3.54 \mathrm{E}-1$ & & 4/29/09 & 9D23001 & AGG-RRL-001 \\
\hline 14391-16-3 & Europium-155 & $<5.53 \mathrm{E}-1$ & pCi/g dry & $5.53 \mathrm{E}-1$ & & $4 / 29 / 09$ & 9D23001 & AGG-RRL-001 \\
\hline HEIS No. & B1YM92 & \multicolumn{4}{|c|}{ Lab ID: $\quad 0902002-12$} & & & \\
\hline $10198-40-0$ & Cobalt- 60 & $<1.84 \mathrm{E}-1$ & pCi/g dry & $1.84 \mathrm{E}-1$ & & $4 / 29 / 09$ & 9D23001 & AGG-RRL-001 \\
\hline $10045-97-3$ & Cesium-137 & $<2.17 \mathrm{E}-1$ & pCi/g dry & $2.17 \mathrm{E}-1$ & & $4 / 29 / 09$ & 9D23001 & AGG-RRL-001 \\
\hline $14683-23-9$ & Europium-152 & $<8.09 \mathrm{E}-1$ & pCi/g dry & $8.09 \mathrm{E}-1$ & & $4 / 29 / 09$ & 9D23001 & AGG-RRL-001 \\
\hline $15585-10-1$ & Europium-154 & $<4.80 \mathrm{E}-1$ & pCi/g dry & $4.80 \mathrm{E}-1$ & & $4 / 29 / 09$ & 9D23001 & AGG-RRL-001 \\
\hline 14391-16-3 & Europium-155 & $<8.09 \mathrm{E}-1$ & pCi/g dry & $8.09 \mathrm{E}-1$ & & $4 / 29 / 09$ & 9D23001 & AGG-RRL-001 \\
\hline HEIS No. & B1YM93 & \multicolumn{4}{|c|}{ Lab ID: $\quad 0902002-13$} & & & \\
\hline $10198-40-0$ & Cobalt-60 & $<1.33 \mathrm{E}-1$ & pCi/g dry & $1.33 \mathrm{E}-1$ & & $4 / 29 / 09$ & 9D23001 & AGG-RRL-001 \\
\hline $10045-97-3$ & Cesium-137 & $<1.58 \mathrm{E}-1$ & pCi/g dry & $1.58 \mathrm{E}-1$ & & $4 / 29 / 09$ & 9D23001 & AGG-RRL-001 \\
\hline $14683-23-9$ & Europium-152 & $<5.62 \mathrm{E}-1$ & $\mathrm{pCi} / \mathrm{g}$ dry & $5.62 \mathrm{E}-1$ & & 4/29/09 & 9D23001 & AGG-RRL-001 \\
\hline $15585-10-1$ & Europium-154 & $<3.43 \mathrm{E}-1$ & pCi/g dry & $3.43 \mathrm{E}-1$ & & $4 / 29 / 09$ & 9D23001 & AGG-RRL-001 \\
\hline $14391-16-3$ & Europium-155 & $<5.47 \mathrm{E}-1$ & pCi/g dry & $5.47 \mathrm{E}-1$ & & 4/29/09 & 9D23001 & AGG-RRL-001 \\
\hline HEIS No. & B1YNV5 & \multicolumn{4}{|c|}{ Lab ID: $\quad 0902002-14$} & & & \\
\hline $10198-40-0$ & Cobalt -60 & $<1.98 \mathrm{E}-1$ & pCi/g dry & $1.98 \mathrm{E}-1$ & & 4/29/09 & 9D23001 & AGG-RRL-001 \\
\hline $10045-97-3$ & Cesium-137 & $<2.25 \mathrm{E}-1$ & pCi/g dry & $2.25 \mathrm{E}-1$ & & $4 / 29 / 09$ & 9D23001 & AGG-RRL-001 \\
\hline $14683-23-9$ & Europium-152 & $<8.38 \mathrm{E}-1$ & pCi/g dry & $8.38 \mathrm{E}-1$ & & $4 / 29 / 09$ & 9D23001 & AGG-RRL-001 \\
\hline $15585-10-1$ & Europium-154 & $<4.85 \mathrm{E}-1$ & pCi/g dry & $4.85 \mathrm{E}-1$ & & 4/29/09 & 9D23001 & AGG-RRL-001 \\
\hline $14391-16-3$ & Europium-155 & $<8.06 \mathrm{E}-1$ & pCi/g dry & $8.06 \mathrm{E}-1$ & & $4 / 29 / 09$ & 9D23001 & AGG-RRL-001 \\
\hline HEIS No. & B1YD76 & \multicolumn{4}{|c|}{ Lab ID: $\quad 0902002-15$} & & & \\
\hline $10198-40-0$ & Cobalt-60 & $<1.58 \mathrm{E}-1$ & pCi/g dry & $1.58 \mathrm{E}-1$ & & 6/03/09 & 9F03001 & AGG-RRL-001 \\
\hline $10045-97-3$ & Cesium-137 & $<1.90 \mathrm{E}-1$ & pCi/g dry & $1.90 \mathrm{E}-1$ & & 6/03/09 & 9F03001 & AGG-RRL-001 \\
\hline $14683-23-9$ & Europium-152 & $<6.58 \mathrm{E}-1$ & pCi/g dry & $6.58 \mathrm{E}-1$ & & 6/03/09 & 9F03001 & AGG-RRL-001 \\
\hline $15585-10-1$ & Europium-154 & $<4.10 \mathrm{E}-1$ & $\mathrm{pCi} / \mathrm{g}$ dry & $4.10 \mathrm{E}-1$ & & 6/03/09 & 9F03001 & AGG-RRL-001 \\
\hline $14391-16-3$ & Europium-155 & $<6.48 \mathrm{E}-1$ & pCi/g dry & $6.48 \mathrm{E}-1$ & & 6/03/09 & 9F03001 & AGG-RRL-001 \\
\hline HEIS No. & B1YD88 & \multicolumn{4}{|c|}{ Lab ID: $\quad$ 0902002-16 } & & & \\
\hline $10198-40-0$ & Cobalt- 60 & $<1.70 \mathrm{E}-1$ & pCi/g dry & $1.70 \mathrm{E}-1$ & & 6/03/09 & 9F03001 & AGG-RRL-001 \\
\hline $10045-97-3$ & Cesium-137 & $<2.25 \mathrm{E}-1$ & $\mathrm{pCi} / \mathrm{g}$ dry & $2.25 \mathrm{E}-1$ & & 6/03/09 & 9F03001 & AGG-RRL-001 \\
\hline $14683-23-9$ & Europium-152 & $<8.59 \mathrm{E}-1$ & pCi/g dry & $8.59 \mathrm{E}-1$ & & 6/03/09 & 9F03001 & AGG-RRL-001 \\
\hline $15585-10-1$ & Europium-154 & $<4.90 \mathrm{E}-1$ & pCi/g dry & $4.90 \mathrm{E}-1$ & & 6/03/09 & 9F03001 & AGG-RRL-001 \\
\hline 14391-16-3 & Europium-155 & $<8.04 \mathrm{E}-1$ & pCi/g dry & $8.04 \mathrm{E}-1$ & & 6/03/09 & 9F03001 & AGG-RRL-001 \\
\hline
\end{tabular}




\section{GEA/Soil}

\begin{tabular}{|c|c|c|c|c|c|c|c|c|}
\hline CAS \# & Analyte & Results & Units & MDA & UNC & Analyzed & Batch & Method \\
\hline HEIS No. & B1YD77 & \multicolumn{3}{|c|}{ Lab ID: $\quad$ 0902002-17 } & & & & \\
\hline $10198-40-0$ & Cobalt- 60 & $<1.67 \mathrm{E}-1$ & pCi/g dry & $1.67 \mathrm{E}-1$ & & $6 / 03 / 09$ & 9F03001 & AGG-RRL-001 \\
\hline $10045-97-3$ & Cesium-137 & $<2.12 \mathrm{E}-1$ & pCi/g dry & $2.12 \mathrm{E}-1$ & & 6/03/09 & 9F03001 & AGG-RRL-001 \\
\hline $14683-23-9$ & Europium-152 & $<7.12 \mathrm{E}-1$ & pCi/g dry & $7.12 \mathrm{E}-1$ & & 6/03/09 & 9F03001 & AGG-RRL-001 \\
\hline $15585-10-1$ & Europium-154 & $<4.53 \mathrm{E}-1$ & pCi/g dry & $4.53 \mathrm{E}-1$ & & $6 / 03 / 09$ & 9F03001 & AGG-RRL-001 \\
\hline $14391-16-3$ & Europium-155 & $<6.87 \mathrm{E}-1$ & pCi/g dry & $6.87 \mathrm{E}-1$ & & $6 / 03 / 09$ & 9F03001 & AGG-RRL-001 \\
\hline HEIS No. & B1YD78 & \multicolumn{3}{|c|}{ Lab ID: $\quad$ 0902002-18 } & & & & \\
\hline $10198-40-0$ & Cobalt- 60 & $<2.53 \mathrm{E}-1$ & pCi/g dry & $2.53 \mathrm{E}-1$ & & $6 / 03 / 09$ & 9F03001 & AGG-RRL-001 \\
\hline $10045-97-3$ & Cesium-137 & $<2.65 \mathrm{E}-1$ & pCi/g dry & $2.65 \mathrm{E}-1$ & & $6 / 03 / 09$ & 9F03001 & AGG-RRL-001 \\
\hline $14683-23-9$ & Europium-152 & $<1.00 \mathrm{E} 0$ & pCi/g dry & $1.00 \mathrm{E} 0$ & & $6 / 03 / 09$ & 9F03001 & AGG-RRL-001 \\
\hline $15585-10-1$ & Europium-154 & $<5.72 \mathrm{E}-1$ & pCi/g dry & $5.72 \mathrm{E}-1$ & & 6/03/09 & 9F03001 & AGG-RRL-001 \\
\hline $14391-16-3$ & Europium-155 & $<9.57 \mathrm{E}-1$ & pCi/g dry & $9.57 \mathrm{E}-1$ & & 6/03/09 & 9F03001 & AGG-RRL-001 \\
\hline HEIS No. & B1YD79 & \multicolumn{3}{|c|}{ Lab ID: $\quad$ 0902002-19 } & & & & \\
\hline $10198-40-0$ & Cobalt-60 & $<2.10 \mathrm{E}-1$ & pCi/g dry & $2.10 \mathrm{E}-1$ & & 6/03/09 & 9F03001 & AGG-RRL-001 \\
\hline $10045-97-3$ & Cesium-137 & $<2.66 \mathrm{E}-1$ & pCi/g dry & $2.66 \mathrm{E}-1$ & & $6 / 03 / 09$ & 9F03001 & AGG-RRL-001 \\
\hline 14683-23-9 & Europium-152 & $<9.34 \mathrm{E}-1$ & pCi/g dry & $9.34 \mathrm{E}-1$ & & 6/03/09 & 9F03001 & AGG-RRL-001 \\
\hline $15585-10-1$ & Europium-154 & $<5.87 \mathrm{E}-1$ & pCi/g dry & $5.87 \mathrm{E}-1$ & & $6 / 03 / 09$ & 9F03001 & AGG-RRL-001 \\
\hline $14391-16-3$ & Europium-155 & $<8.86 \mathrm{E}-1$ & pCi/g dry & $8.86 \mathrm{E}-1$ & & 6/03/09 & 9F03001 & AGG-RRL-001 \\
\hline HEIS No. & B1YND9 & \multicolumn{3}{|c|}{ Lab ID: $\quad$ 0902002-20 } & & & & \\
\hline $10198-40-0$ & Cobalt- 60 & $<2.21 \mathrm{E}-1$ & pCi/g dry & $2.21 \mathrm{E}-1$ & & 6/03/09 & 9F03001 & AGG-RRL-001 \\
\hline $10045-97-3$ & Cesium-137 & $<2.45 \mathrm{E}-1$ & pCi/g dry & $2.45 \mathrm{E}-1$ & & $6 / 03 / 09$ & 9F03001 & AGG-RRL-001 \\
\hline $14683-23-9$ & Europium-152 & $<9.38 \mathrm{E}-1$ & pCi/g dry & $9.38 \mathrm{E}-1$ & & 6/03/09 & 9F03001 & AGG-RRL-001 \\
\hline $15585-10-1$ & Europium-154 & $<5.39 \mathrm{E}-1$ & pCi/g dry & $5.39 \mathrm{E}-1$ & & $6 / 03 / 09$ & 9F03001 & AGG-RRL-001 \\
\hline $14391-16-3$ & Europium-155 & $<8.96 \mathrm{E}-1$ & pCi/g dry & 8.96E-1 & & $6 / 03 / 09$ & 9F03001 & AGG-RRL-001 \\
\hline HEIS No. & B1YNF0 & \multicolumn{3}{|c|}{ Lab ID: $\quad$ 0902002-21 } & & & & \\
\hline $10198-40-0$ & Cobalt- 60 & $<2.02 \mathrm{E}-1$ & pCi/g dry & $2.02 \mathrm{E}-1$ & & $6 / 04 / 09$ & 9F03001 & AGG-RRL-001 \\
\hline $10045-97-3$ & Cesium-137 & $<2.46 \mathrm{E}-1$ & pCi/g dry & $2.46 \mathrm{E}-1$ & & $6 / 04 / 09$ & 9F03001 & AGG-RRL-001 \\
\hline $14683-23-9$ & Europium-152 & $<8.70 \mathrm{E}-1$ & pCi/g dry & $8.70 \mathrm{E}-1$ & & $6 / 04 / 09$ & 9F03001 & AGG-RRL-001 \\
\hline $15585-10-1$ & Europium-154 & $<5.30 \mathrm{E}-1$ & pCi/g dry & $5.30 \mathrm{E}-1$ & & $6 / 04 / 09$ & 9F03001 & AGG-RRL-001 \\
\hline $14391-16-3$ & Europium-155 & $<8.17 \mathrm{E}-1$ & pCi/g dry & $8.17 \mathrm{E}-1$ & & $6 / 04 / 09$ & 9F03001 & AGG-RRL-001 \\
\hline HEIS No. & B1YNF1 & \multicolumn{3}{|c|}{ Lab ID: $\quad$ 0902002-22 } & & & & \\
\hline $10198-40-0$ & Cobalt- 60 & $<2.15 \mathrm{E}-1$ & pCi/g dry & $2.15 \mathrm{E}-1$ & & $6 / 04 / 09$ & 9F03001 & AGG-RRL-001 \\
\hline $10045-97-3$ & Cesium-137 & $<2.67 \mathrm{E}-1$ & pCi/g dry & $2.67 \mathrm{E}-1$ & & $6 / 04 / 09$ & 9F03001 & AGG-RRL-001 \\
\hline $14683-23-9$ & Europium-152 & $<1.03 \mathrm{E} 0$ & pCi/g dry & $1.03 \mathrm{E} 0$ & & 6/04/09 & 9F03001 & AGG-RRL-001 \\
\hline $15585-10-1$ & Europium-154 & $<5.70 \mathrm{E}-1$ & pCi/g dry & $5.70 \mathrm{E}-1$ & & $6 / 04 / 09$ & 9F03001 & AGG-RRL-001 \\
\hline 14391-16-3 & Europium-155 & $<9.35 \mathrm{E}-1$ & pCi/g dry & $9.35 \mathrm{E}-1$ & & $6 / 04 / 09$ & 9F03001 & AGG-RRL-001 \\
\hline HEIS No. & B1YD80 & \multicolumn{3}{|c|}{ Lab ID: $\quad 0902002-23$} & & & & \\
\hline $10198-40-0$ & Cobalt- 60 & $<1.94 \mathrm{E}-1$ & pCi/g dry & $1.94 \mathrm{E}-1$ & & $6 / 04 / 09$ & 9F03001 & AGG-RRL-001 \\
\hline $10045-97-3$ & Cesium-137 & $<2.46 \mathrm{E}-1$ & pCi/g dry & $2.46 \mathrm{E}-1$ & & $6 / 04 / 09$ & 9F03001 & AGG-RRL-001 \\
\hline $14683-23-9$ & Europium-152 & $<8.26 \mathrm{E}-1$ & pCi/g dry & $8.26 \mathrm{E}-1$ & & $6 / 04 / 09$ & 9F03001 & AGG-RRL-001 \\
\hline $15585-10-1$ & Europium-154 & $<5.17 \mathrm{E}-1$ & pCi/g dry & $5.17 \mathrm{E}-1$ & & $6 / 04 / 09$ & 9F03001 & AGG-RRL-001 \\
\hline $14391-16-3$ & Europium-155 & $<7.96 \mathrm{E}-1$ & pCi/g dry & $7.96 \mathrm{E}-1$ & & $6 / 04 / 09$ & 9F03001 & AGG-RRL-001 \\
\hline HEIS No. & B1YD81 & \multicolumn{3}{|c|}{ Lab ID: $\quad$ 0902002-24 } & & & & \\
\hline $10198-40-0$ & Cobalt- 60 & $<1.91 \mathrm{E}-1$ & pCi/g dry & $1.91 \mathrm{E}-1$ & & $6 / 04 / 09$ & 9F03001 & AGG-RRL-001 \\
\hline $10045-97-3$ & Cesium-137 & $<2.23 \mathrm{E}-1$ & pCi/g dry & $2.23 \mathrm{E}-1$ & & 6/04/09 & 9F03001 & AGG-RRL-001 \\
\hline $14683-23-9$ & Europium-152 & $<8.47 \mathrm{E}-1$ & pCi/g dry & $8.47 \mathrm{E}-1$ & & 6/04/09 & 9F03001 & AGG-RRL-001 \\
\hline $15585-10-1$ & Europium-154 & $<4.81 \mathrm{E}-1$ & pCi/g dry & $4.81 \mathrm{E}-1$ & & 6/04/09 & 9F03001 & AGG-RRL-001 \\
\hline 14391-16-3 & Europium-155 & $<8.09 \mathrm{E}-1$ & pCi/g dry & $8.09 \mathrm{E}-1$ & & $6 / 04 / 09$ & 9F03001 & AGG-RRL-001 \\
\hline
\end{tabular}




\section{GEA/Soil}

\begin{tabular}{|c|c|c|c|c|c|c|c|c|}
\hline CAS \# & Analyte & Results & Units & MDA & UNC & Analyzed & Batch & Method \\
\hline HEIS No. & B1YD82 & \multicolumn{3}{|c|}{ Lab ID: $\quad 0902002-25$} & & & & \\
\hline $10198-40-0$ & Cobalt-60 & $<3.64 \mathrm{E}-1$ & pCi/g dry & $3.64 \mathrm{E}-1$ & & 6/05/09 & 9F03001 & AGG-RRL-001 \\
\hline $10045-97-3$ & Cesium-137 & $<4.43 \mathrm{E}-1$ & pCi/g dry & $4.43 \mathrm{E}-1$ & & $6 / 05 / 09$ & 9F03001 & AGG-RRL-001 \\
\hline $14683-23-9$ & Europium-152 & $<1.54 \mathrm{E} 0$ & pCi/g dry & $1.54 \mathrm{E} 0$ & & $6 / 05 / 09$ & 9F03001 & AGG-RRL-001 \\
\hline $15585-10-1$ & Europium-154 & $<9.84 \mathrm{E}-1$ & pCi/g dry & $9.84 \mathrm{E}-1$ & & $6 / 05 / 09$ & 9F03001 & AGG-RRL-001 \\
\hline $14391-16-3$ & Europium-155 & $<1.63 \mathrm{E} 0$ & pCi/g dry & $1.63 \mathrm{E} 0$ & & $6 / 05 / 09$ & 9F03001 & AGG-RRL-001 \\
\hline HEIS No. & B1YNF2 & \multicolumn{3}{|c|}{ Lab ID: $\quad 0902002-26$} & & & & \\
\hline $10198-40-0$ & Cobalt- 60 & $<2.01 \mathrm{E}-1$ & pCi/g dry & $2.01 \mathrm{E}-1$ & & $6 / 05 / 09$ & 9F03001 & AGG-RRL-001 \\
\hline $10045-97-3$ & Cesium-137 & $<2.55 \mathrm{E}-1$ & pCi/g dry & $2.55 \mathrm{E}-1$ & & 6/05/09 & 9F03001 & AGG-RRL-001 \\
\hline $14683-23-9$ & Europium-152 & $<9.62 \mathrm{E}-1$ & pCi/g dry & $9.62 \mathrm{E}-1$ & & $6 / 05 / 09$ & 9F03001 & AGG-RRL-001 \\
\hline $15585-10-1$ & Europium-154 & $<5.47 \mathrm{E}-1$ & pCi/g dry & $5.47 \mathrm{E}-1$ & & $6 / 05 / 09$ & 9F03001 & AGG-RRL-001 \\
\hline $14391-16-3$ & Europium-155 & $<9.06 \mathrm{E}-1$ & pCi/g dry & $9.06 \mathrm{E}-1$ & & $6 / 05 / 09$ & 9F03001 & AGG-RRL-001 \\
\hline HEIS No. & B1YNF3 & \multicolumn{3}{|c|}{ Lab ID: $\quad$ 0902002-27 } & & & & \\
\hline $10198-40-0$ & Cobalt- 60 & $<2.12 \mathrm{E}-1$ & pCi/g dry & $2.12 \mathrm{E}-1$ & & 6/08/09 & 9F03001 & AGG-RRL-001 \\
\hline $10045-97-3$ & Cesium-137 & $<2.62 \mathrm{E}-1$ & $\mathrm{pCi} / \mathrm{g}$ dry & $2.62 \mathrm{E}-1$ & & 6/08/09 & 9F03001 & AGG-RRL-001 \\
\hline $14683-23-9$ & Europium-152 & $<8.75 \mathrm{E}-1$ & pCi/g dry & $8.75 \mathrm{E}-1$ & & 6/08/09 & 9F03001 & AGG-RRL-001 \\
\hline $15585-10-1$ & Europium-154 & $<5.46 \mathrm{E}-1$ & pCi/g dry & $5.46 \mathrm{E}-1$ & & 6/08/09 & 9F03001 & AGG-RRL-001 \\
\hline $14391-16-3$ & Europium-155 & $<8.23 \mathrm{E}-1$ & pCi/g dry & $8.23 \mathrm{E}-1$ & & 6/08/09 & 9F03001 & AGG-RRL-001 \\
\hline HEIS No. & B1YNF4 & \multicolumn{3}{|c|}{ Lab ID: $\quad 0902002-28$} & & & & \\
\hline $10198-40-0$ & Cobalt- 60 & $<3.13 \mathrm{E}-1$ & pCi/g dry & $3.13 \mathrm{E}-1$ & & 6/08/09 & 9F03001 & AGG-RRL-001 \\
\hline $10045-97-3$ & Cesium-137 & $<3.47 \mathrm{E}-1$ & pCi/g dry & $3.47 \mathrm{E}-1$ & & $6 / 08 / 09$ & 9F03001 & AGG-RRL-001 \\
\hline $14683-23-9$ & Europium-152 & $<1.34 \mathrm{E} 0$ & pCi/g dry & $1.34 \mathrm{E} 0$ & & 6/08/09 & 9F03001 & AGG-RRL-001 \\
\hline $15585-10-1$ & Europium-154 & $<7.25 \mathrm{E}-1$ & pCi/g dry & $7.25 \mathrm{E}-1$ & & 6/08/09 & 9F03001 & AGG-RRL-001 \\
\hline 14391-16-3 & Europium-155 & $<1.28 \mathrm{E} 0$ & pCi/g dry & $1.28 \mathrm{E} 0$ & & 6/08/09 & 9F03001 & AGG-RRL-001 \\
\hline HEIS No. & B1YNF5 & \multicolumn{3}{|c|}{ Lab ID: $\quad 0902002-29$} & & & & \\
\hline $10198-40-0$ & Cobalt-60 & $<3.19 \mathrm{E}-1$ & pCi/g dry & $3.19 \mathrm{E}-1$ & & 6/08/09 & 9F03001 & AGG-RRL-001 \\
\hline $10045-97-3$ & Cesium-137 & $<3.68 \mathrm{E}-1$ & pCi/g dry & $3.68 \mathrm{E}-1$ & & 6/08/09 & 9F03001 & AGG-RRL-001 \\
\hline $14683-23-9$ & Europium-152 & $<1.30 \mathrm{E} 0$ & $\mathrm{pCi} / \mathrm{g}$ dry & $1.30 \mathrm{E} 0$ & & 6/08/09 & 9F03001 & AGG-RRL-001 \\
\hline $15585-10-1$ & Europium-154 & $<7.96 \mathrm{E}-1$ & pCi/g dry & $7.96 \mathrm{E}-1$ & & 6/08/09 & 9F03001 & AGG-RRL-001 \\
\hline $14391-16-3$ & Europium-155 & $<1.24 \mathrm{E} 0$ & pCi/g dry & $1.24 \mathrm{E} 0$ & & 6/08/09 & 9F03001 & AGG-RRL-001 \\
\hline HEIS No. & B1YNF6 & \multicolumn{3}{|c|}{ Lab ID: $\quad 0902002-30$} & & & & \\
\hline $10198-40-0$ & Cobalt -60 & $<2.46 \mathrm{E}-1$ & pCi/g dry & $2.46 \mathrm{E}-1$ & & 6/08/09 & 9F03001 & AGG-RRL-001 \\
\hline $10045-97-3$ & Cesium-137 & $<3.03 \mathrm{E}-1$ & pCi/g dry & $3.03 \mathrm{E}-1$ & & 6/08/09 & 9F03001 & AGG-RRL-001 \\
\hline $14683-23-9$ & Europium-152 & $<1.16 \mathrm{E} 0$ & pCi/g dry & $1.16 \mathrm{E} 0$ & & $6 / 08 / 09$ & 9F03001 & AGG-RRL-001 \\
\hline $15585-10-1$ & Europium-154 & $<6.54 \mathrm{E}-1$ & pCi/g dry & $6.54 \mathrm{E}-1$ & & 6/08/09 & 9F03001 & AGG-RRL-001 \\
\hline $14391-16-3$ & Europium-155 & $<1.12 \mathrm{E} 0$ & pCi/g dry & $1.12 \mathrm{E} 0$ & & $6 / 08 / 09$ & 9F03001 & AGG-RRL-001 \\
\hline HEIS No. & B1YD83 & \multicolumn{3}{|c|}{ Lab ID: $\quad 0902002-31$} & & & & \\
\hline $10198-40-0$ & Cobalt-60 & $<1.82 \mathrm{E}-1$ & pCi/g dry & $1.82 \mathrm{E}-1$ & & $6 / 08 / 09$ & 9F03001 & AGG-RRL-001 \\
\hline $10045-97-3$ & Cesium-137 & $<2.35 \mathrm{E}-1$ & pCi/g dry & $2.35 \mathrm{E}-1$ & & 6/08/09 & 9F03001 & AGG-RRL-001 \\
\hline $14683-23-9$ & Europium-152 & $<8.04 \mathrm{E}-1$ & pCi/g dry & 8.04E-1 & & 6/08/09 & 9F03001 & AGG-RRL-001 \\
\hline $15585-10-1$ & Europium-154 & $<5.24 \mathrm{E}-1$ & $\mathrm{pCi} / \mathrm{g}$ dry & $5.24 \mathrm{E}-1$ & & 6/08/09 & 9F03001 & AGG-RRL-001 \\
\hline $14391-16-3$ & Europium-155 & $<8.10 \mathrm{E}-1$ & pCi/g dry & $8.10 \mathrm{E}-1$ & & 6/08/09 & 9F03001 & AGG-RRL-001 \\
\hline HEIS No. & B1YM97 & \multicolumn{3}{|c|}{ Lab ID: $\quad 0902002-37$} & & & & \\
\hline $10198-40-0$ & Cobalt- 60 & $<2.05 \mathrm{E}-1$ & pCi/g dry & $2.05 \mathrm{E}-1$ & & $2 / 09 / 10$ & 0B12010 & AGG-RRL-001 \\
\hline $10045-97-3$ & Cesium-137 & $<2.51 \mathrm{E}-1$ & $\mathrm{pCi} / \mathrm{g}$ dry & $2.51 \mathrm{E}-1$ & & $2 / 09 / 10$ & 0B12010 & AGG-RRL-001 \\
\hline $14683-23-9$ & Europium-152 & $<9.85 \mathrm{E}-1$ & pCi/g dry & $9.85 \mathrm{E}-1$ & & $2 / 09 / 10$ & 0B12010 & AGG-RRL-001 \\
\hline $15585-10-1$ & Europium-154 & $<5.76 \mathrm{E}-1$ & pCi/g dry & $5.76 \mathrm{E}-1$ & & $2 / 09 / 10$ & 0B12010 & AGG-RRL-001 \\
\hline $14391-16-3$ & Europium-155 & $<8.75 \mathrm{E}-1$ & pCi/g dry & $8.75 \mathrm{E}-1$ & & $2 / 09 / 10$ & 0B12010 & AGG-RRL-001 \\
\hline
\end{tabular}


Anions by Ion Chromatography - Quality Control

Environmental Science Laboratory

\begin{tabular}{|c|c|c|c|c|c|c|c|c|c|c|}
\hline Analyte & Result & $\begin{array}{r}\text { porting } \\
\text { Limit }\end{array}$ & Units & $\begin{array}{l}\text { Spike } \\
\text { Level }\end{array}$ & $\begin{array}{l}\text { Source } \\
\text { Result }\end{array}$ & $\%$ REC & $\begin{array}{l}\text { \%REC } \\
\text { Limits }\end{array}$ & RPD & $\begin{array}{l}\text { RPD } \\
\text { Limit }\end{array}$ & Notes \\
\hline
\end{tabular}

Batch 9C10004 - 1:1 Water Extract (IC)

\begin{tabular}{|c|c|c|c|c|c|c|c|c|c|c|}
\hline \multirow{2}{*}{$\begin{array}{l}\text { Blank (9C10004-BLK1) } \\
\text { Fluoride }\end{array}$} & \multicolumn{10}{|c|}{ Prepared \& Analyzed: 03/10/09 } \\
\hline & $<2.00 \mathrm{E}-1$ & $2.00 \mathrm{E}-1$ & ug/g wet & & & & & & & \\
\hline Chloride & $<5.00 \mathrm{E}-1$ & $5.00 \mathrm{E}-1$ & $"$ & & & & & & & \\
\hline Nitrite & $<1.00 \mathrm{E} 0$ & $1.00 \mathrm{E} 0$ & $"$ & & & & & & & \\
\hline Nitrate & $<1.00 \mathrm{E} 0$ & $1.00 \mathrm{E} 0$ & $"$ & & & & & & & \\
\hline Sulfate & $<1.50 \mathrm{E} 0$ & $1.50 \mathrm{E} 0$ & $"$ & & & & & & & \\
\hline Phosphate & $<1.50 \mathrm{E} 0$ & $1.50 \mathrm{E} 0$ & $"$ & & & & & & & \\
\hline LCS (9C10004-BS1) & \multicolumn{10}{|c|}{ Prepared \& Analyzed: 03/10/09 } \\
\hline Fluoride & $2.01 \mathrm{E} 0$ & $2.07 \mathrm{E}-1$ & $\mathrm{ug} / \mathrm{g}$ wet & $2.07 \mathrm{E} 0$ & & 97.0 & $80-120$ & & & $\overline{\mathrm{D}}$ \\
\hline Chloride & $5.18 \mathrm{E} 0$ & $5.17 \mathrm{E}-1$ & $"$ & $5.17 \mathrm{E} 0$ & & 100 & $80-120$ & & & $\mathrm{D}$ \\
\hline Nitrite & $9.70 \mathrm{E} 0$ & $1.03 \mathrm{E} 0$ & $"$ & $1.03 \mathrm{E} 1$ & & 93.7 & $80-120$ & & & $\mathrm{D}$ \\
\hline Nitrate & $1.07 \mathrm{E} 1$ & $1.03 \mathrm{E} 0$ & $"$ & $1.03 \mathrm{E} 1$ & & 104 & $80-120$ & & & $\mathrm{D}$ \\
\hline Sulfate & $1.65 \mathrm{E} 1$ & $1.55 \mathrm{E} 0$ & $"$ & $1.55 \mathrm{E} 1$ & & 106 & $80-120$ & & & $\mathrm{D}$ \\
\hline Phosphate & $1.56 \mathrm{E} 1$ & $1.55 \mathrm{E} 0$ & $"$ & $1.55 \mathrm{E} 1$ & & 100 & $80-120$ & & & $\mathrm{D}$ \\
\hline Duplicate (9C10004-DUP1) & \multicolumn{3}{|c|}{ Source: 0902002-01 } & \multicolumn{7}{|c|}{ Prepared \& Analyzed: 03/10/09 } \\
\hline Fluoride & $4.90 \mathrm{E}-1$ & $2.16 \mathrm{E}-1$ & ug/g dry & & $5.50 \mathrm{E}-1$ & & & 11.6 & 35 & $\mathrm{D}$ \\
\hline Chloride & $2.35 \mathrm{E} 0$ & $5.40 \mathrm{E}-1$ & $"$ & & $3.83 \mathrm{E} 0$ & & & 47.7 & 35 & $\mathrm{D}$ \\
\hline Nitrite & $<1.08 \mathrm{E} 0$ & $1.08 \mathrm{E} 0$ & $"$ & & ND & & & & 35 & \\
\hline Nitrate & $2.39 \mathrm{E} 0$ & $1.08 \mathrm{E} 0$ & $"$ & & $2.92 \mathrm{E} 0$ & & & 19.8 & 35 & $\mathrm{D}$ \\
\hline Sulfate & $1.19 \mathrm{E} 1$ & $1.62 \mathrm{E} 0$ & $"$ & & $1.51 \mathrm{E} 1$ & & & 23.8 & 35 & $\mathrm{D}$ \\
\hline Phosphate & $<1.62 \mathrm{E} 0$ & $1.62 \mathrm{E} 0$ & $"$ & & ND & & & & 35 & \\
\hline Post Spike (9C10004-PS1) & \multicolumn{3}{|c|}{ Source: 0902002-01 } & \multicolumn{7}{|c|}{ Prepared \& Analyzed: 03/10/09 } \\
\hline Fluoride & $1.39 \mathrm{E} 0$ & N/A & $\mathrm{ug} / \mathrm{mL}$ & $7.69 \mathrm{E}-1$ & $5.10 \mathrm{E}-1$ & 115 & $75-125$ & & & $\bar{D}$ \\
\hline Chloride & $5.35 \mathrm{E} 0$ & N/A & $"$ & $1.92 \mathrm{E} 0$ & $3.55 \mathrm{E} 0$ & 93.4 & $75-125$ & & & $\mathrm{D}$ \\
\hline Nitrite & $3.83 \mathrm{E} 0$ & N/A & $"$ & $3.85 \mathrm{E} 0$ & ND & 99.7 & $75-125$ & & & $\mathrm{D}$ \\
\hline Nitrate & $6.60 \mathrm{E} 0$ & N/A & $"$ & $3.85 \mathrm{E} 0$ & $2.71 \mathrm{E} 0$ & 101 & $75-125$ & & & $\mathrm{D}$ \\
\hline Sulfate & $1.89 \mathrm{E} 1$ & N/A & $"$ & $5.77 \mathrm{E} 0$ & $1.40 \mathrm{E} 1$ & 84.4 & $75-125$ & & & $\mathrm{D}$ \\
\hline Phosphate & $5.61 \mathrm{E} 0$ & N/A & $"$ & $5.77 \mathrm{E} 0$ & ND & 97.2 & $75-125$ & & & $\mathrm{D}$ \\
\hline
\end{tabular}


Anions by Ion Chromatography - Quality Control

Environmental Science Laboratory

\begin{tabular}{|lrrrrrrrrrrrr}
\hline & & Reporting & & Spike & Source & & & $\%$ REC & & RPD \\
Analyte & Result & Limit & Units & Level & Result & $\%$ REC & Limits & RPD & Limit & Notes \\
\hline
\end{tabular}

Batch 9C25004 - 1:1 Water Extract (IC)

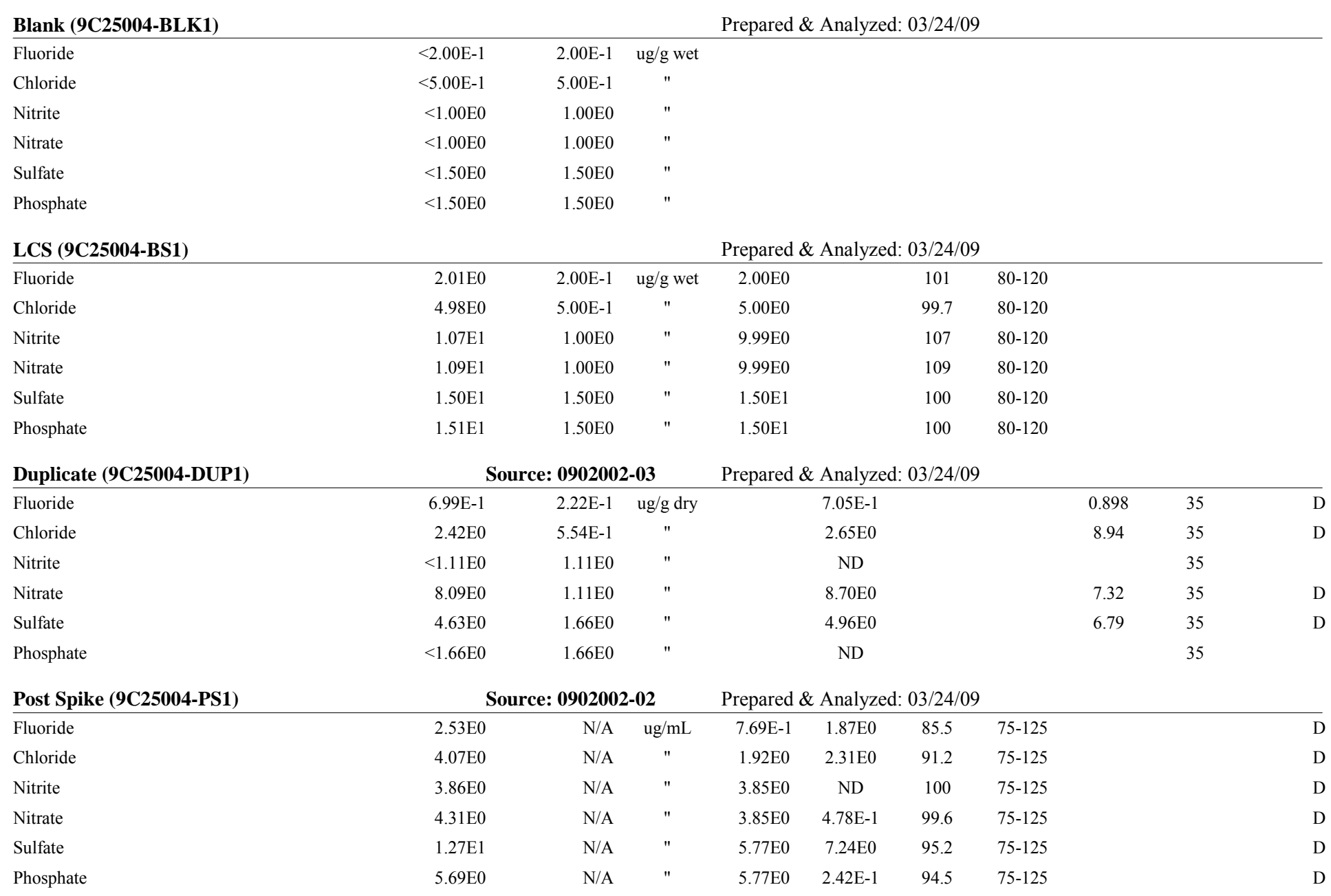


Anions by Ion Chromatography - Quality Control

Environmental Science Laboratory

\begin{tabular}{|lrrrrrrrrrrrr}
\hline & & Reporting & & Spike & Source & & & $\%$ REC & & RPD \\
Analyte & Result & Limit & Units & Level & Result & $\%$ REC & Limits & RPD & Limit & Notes \\
\hline
\end{tabular}

Batch 9D14001 - 1:1 Water Extract (IC)

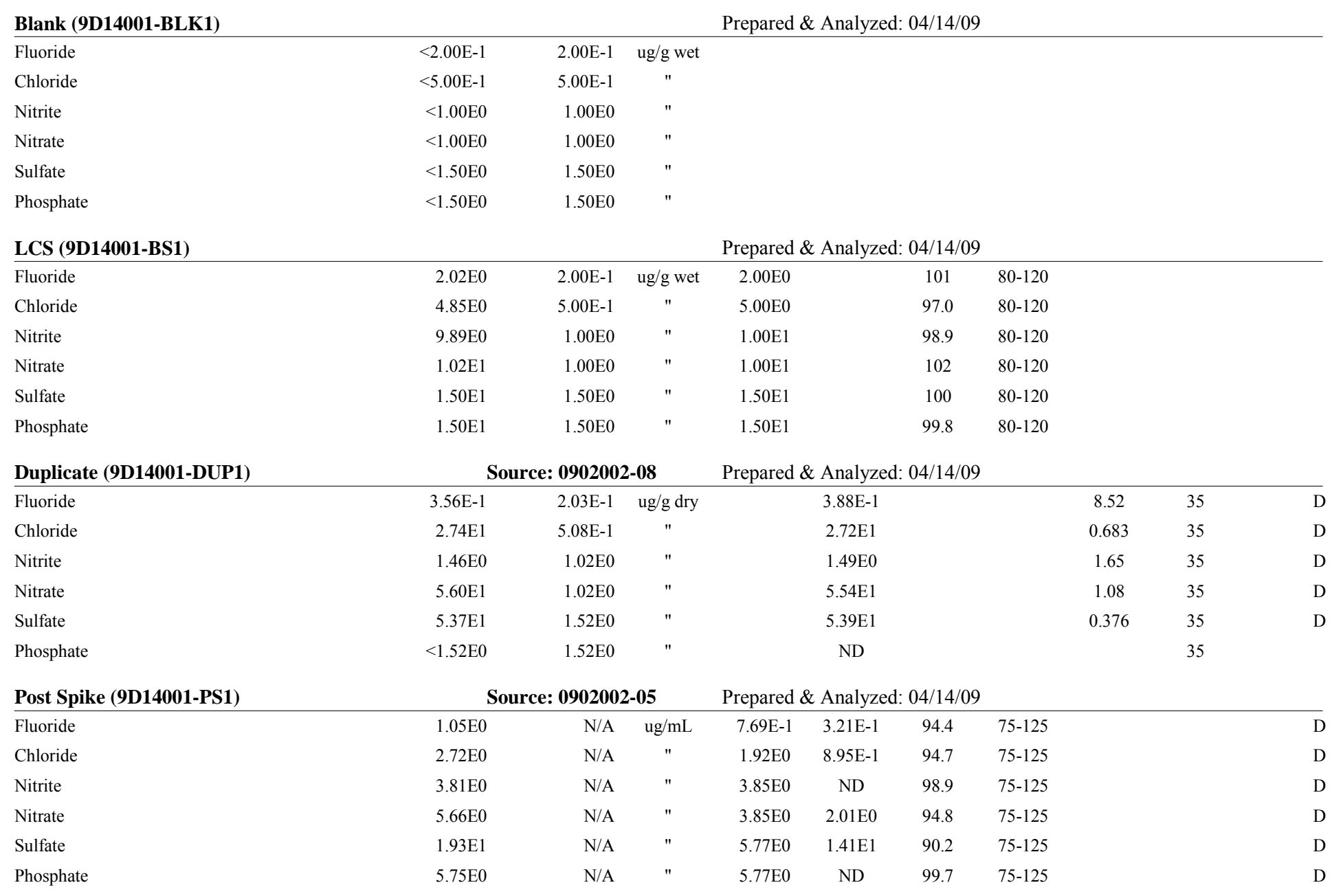


Anions by Ion Chromatography - Quality Control

Environmental Science Laboratory

\begin{tabular}{|lrrrrrrrrrrrr}
\hline & & Reporting & & Spike & Source & & & $\%$ REC & & RPD \\
Analyte & Result & Limit & Units & Level & Result & $\%$ REC & Limits & RPD & Limit & Notes \\
\hline
\end{tabular}

Batch 9D21001 - 1:1 Water Extract (IC)

\begin{tabular}{|c|c|c|c|c|c|c|c|c|c|c|}
\hline \multirow{2}{*}{$\begin{array}{l}\text { Blank (9D21001-BLK1) } \\
\text { Fluoride }\end{array}$} & \multicolumn{10}{|c|}{ Prepared \& Analyzed: 04/21/09 } \\
\hline & $<2.00 \mathrm{E}-1$ & $2.00 \mathrm{E}-1$ & ug/g wet & & & & & & & \\
\hline Chloride & $<5.00 \mathrm{E}-1$ & $5.00 \mathrm{E}-1$ & $"$ & & & & & & & \\
\hline Nitrite & $<1.00 \mathrm{E} 0$ & $1.00 \mathrm{E} 0$ & $"$ & & & & & & & \\
\hline Nitrate & $<1.00 \mathrm{E} 0$ & $1.00 \mathrm{E} 0$ & $"$ & & & & & & & \\
\hline Sulfate & $<1.50 \mathrm{E} 0$ & $1.50 \mathrm{E} 0$ & $"$ & & & & & & & \\
\hline Phosphate & $<1.50 \mathrm{E} 0$ & $1.50 \mathrm{E} 0$ & $"$ & & & & & & & \\
\hline LCS (9D21001-BS1) & \multicolumn{10}{|c|}{ Prepared \& Analyzed: 04/21/09 } \\
\hline Fluoride & $1.98 \mathrm{E} 0$ & $2.00 \mathrm{E}-1$ & $\mathrm{ug} / \mathrm{g}$ wet & $2.00 \mathrm{E} 0$ & & 99.1 & $80-120$ & & & \\
\hline Chloride & $5.01 \mathrm{E} 0$ & $5.00 \mathrm{E}-1$ & $"$ & $5.00 \mathrm{E} 0$ & & 100 & $80-120$ & & & \\
\hline Nitrite & $1.01 \mathrm{E} 1$ & $1.00 \mathrm{E} 0$ & $"$ & $1.00 \mathrm{E} 1$ & & 101 & $80-120$ & & & \\
\hline Nitrate & $1.03 \mathrm{E} 1$ & $1.00 \mathrm{E} 0$ & $"$ & $1.00 \mathrm{E} 1$ & & 103 & $80-120$ & & & \\
\hline Sulfate & $1.52 \mathrm{E} 1$ & $1.50 \mathrm{E} 0$ & $"$ & $1.50 \mathrm{E} 1$ & & 101 & $80-120$ & & & \\
\hline Phosphate & $1.50 \mathrm{E} 1$ & $1.50 \mathrm{E} 0$ & $"$ & $1.50 \mathrm{E} 1$ & & 99.7 & $80-120$ & & & \\
\hline Duplicate (9D21001-DUP1) & \multicolumn{3}{|c|}{ Source: 0902002-12 } & \multicolumn{7}{|c|}{ Prepared \& Analyzed: 04/21/09 } \\
\hline Fluoride & $5.71 \mathrm{E}-1$ & $2.02 \mathrm{E}-1$ & ug/g dry & & $5.59 \mathrm{E}-1$ & & & 2.15 & 35 & $\overline{\mathrm{D}}$ \\
\hline Chloride & $1.35 \mathrm{E} 1$ & $5.04 \mathrm{E}-1$ & $"$ & & $1.36 \mathrm{E} 1$ & & & 0.783 & 35 & $\mathrm{D}$ \\
\hline Nitrite & $<1.01 \mathrm{E} 0$ & $1.01 \mathrm{E} 0$ & $"$ & & ND & & & & 35 & \\
\hline Nitrate & $4.04 \mathrm{E} 1$ & $1.01 \mathrm{E} 0$ & $"$ & & $3.91 \mathrm{E} 1$ & & & 3.19 & 35 & $\mathrm{D}$ \\
\hline Sulfate & $2.31 \mathrm{E} 1$ & $1.51 \mathrm{E} 0$ & $"$ & & $2.23 \mathrm{E} 1$ & & & 3.26 & 35 & $\mathrm{D}$ \\
\hline Phosphate & $<1.51 \mathrm{E} 0$ & $1.51 \mathrm{E} 0$ & $"$ & & ND & & & & 35 & \\
\hline Post Spike (9D21001-PS1) & \multicolumn{3}{|c|}{ Source: 0902002-12 } & \multicolumn{7}{|c|}{ Prepared \& Analyzed: 04/21/09 } \\
\hline Fluoride & $1.35 \mathrm{E} 0$ & N/A & $\mathrm{ug} / \mathrm{mL}$ & $7.69 \mathrm{E}-1$ & $5.55 \mathrm{E}-1$ & 103 & $75-125$ & & & $\overline{\mathrm{D}}$ \\
\hline Chloride & $1.53 \mathrm{E} 1$ & N/A & $"$ & $1.92 \mathrm{E} 0$ & $1.35 \mathrm{E} 1$ & 95.1 & $75-125$ & & & $\mathrm{D}$ \\
\hline Nitrite & $3.88 \mathrm{E} 0$ & N/A & $"$ & $3.85 \mathrm{E} 0$ & ND & 101 & $75-125$ & & & $\mathrm{D}$ \\
\hline Nitrate & $4.18 \mathrm{E} 1$ & N/A & $"$ & $3.85 \mathrm{E} 0$ & $3.89 \mathrm{E} 1$ & 76.5 & $75-125$ & & & $\mathrm{D}$ \\
\hline Sulfate & $2.80 \mathrm{E} 1$ & N/A & $"$ & $5.77 \mathrm{E} 0$ & $2.22 \mathrm{E} 1$ & 100 & $75-125$ & & & $\mathrm{D}$ \\
\hline Phosphate & 5.67E0 & $\mathrm{N} / \mathrm{A}$ & $"$ & $5.77 \mathrm{E} 0$ & $4.24 \mathrm{E}-1$ & 90.9 & $75-125$ & & & $\mathrm{D}$ \\
\hline
\end{tabular}


Total Metals by PNNL-AGG-ICP-AES/Water Extract - Quality Control

Environmental Science Laboratory

\begin{tabular}{|lrrrrrrrrrrr}
\hline & & Reporting & & Spike & Source & & \%REC & & RPD \\
Analyte & Result & Limit & Units & Level & Result & \%REC & Limits & RPD & Limit & Notes \\
\hline
\end{tabular}

Batch 9E12002 - 1:1 Water Extract (ICP/ICPMS)

\begin{tabular}{|c|c|c|c|c|c|}
\hline Blank (9E12002-BLK1) & & & & Prepared: 03/09/09 & Analyzed: 05/13/09 \\
\hline Aluminum & $<9.37 \mathrm{E}-2$ & $9.37 \mathrm{E}-2$ & ug/g wet & & \\
\hline Boron & $<1.37 \mathrm{E} 0$ & $1.37 \mathrm{E} 0$ & $"$ & & \\
\hline Barium & $<1.24 \mathrm{E}-1$ & $1.24 \mathrm{E}-1$ & $"$ & & \\
\hline Beryllium & $<1.31 \mathrm{E}-2$ & $1.31 \mathrm{E}-2$ & $"$ & & \\
\hline Bismuth & $<3.04 \mathrm{E}-1$ & $3.04 \mathrm{E}-1$ & $"$ & & \\
\hline Calcium & $<4.52 \mathrm{E}-1$ & $4.52 \mathrm{E}-1$ & $"$ & & \\
\hline Cobalt & $<1.31 \mathrm{E}-1$ & $1.31 \mathrm{E}-1$ & $"$ & & \\
\hline Copper & $<8.78 \mathrm{E}-2$ & $8.78 \mathrm{E}-2$ & $"$ & & \\
\hline Iron & $<1.05 \mathrm{E}-1$ & $1.05 \mathrm{E}-1$ & $"$ & & \\
\hline Potassium & $<1.29 \mathrm{E} 0$ & $1.29 \mathrm{E} 0$ & $"$ & & \\
\hline Lithium & $<7.47 \mathrm{E}-1$ & $7.47 \mathrm{E}-1$ & $"$ & & \\
\hline Magnesium & $<4.89 \mathrm{E}-2$ & $4.89 \mathrm{E}-2$ & $"$ & & \\
\hline Manganese & $<6.25 \mathrm{E}-2$ & $6.25 \mathrm{E}-2$ & $"$ & & \\
\hline Molybdenum & $<1.41 \mathrm{E}-1$ & $1.41 \mathrm{E}-1$ & $"$ & & \\
\hline Phosphorus & $<6.03 \mathrm{E}-1$ & $6.03 \mathrm{E}-1$ & $"$ & & \\
\hline Strontium & $<8.02 \mathrm{E}-2$ & $8.02 \mathrm{E}-2$ & $"$ & & \\
\hline Vanadium & $<1.33 \mathrm{E}-1$ & $1.33 \mathrm{E}-1$ & $"$ & & \\
\hline Zinc & $<9.77 \mathrm{E}-2$ & $9.77 \mathrm{E}-2$ & $"$ & & \\
\hline Sodium & $<7.97 \mathrm{E}-1$ & 7.97E-1 & $"$ & & \\
\hline Zirconium & $<1.29 \mathrm{E}-1$ & $1.29 \mathrm{E}-1$ & $"$ & & \\
\hline LCS (9E12002-BS1) & & & & Prepared: 03/09/09 & Analyzed: 05/13/09 \\
\hline Aluminum & $4.80 \mathrm{E} 0$ & $9.37 \mathrm{E}-2$ & ug/g wet & $5.00 \mathrm{E} 0$ & $80-120$ \\
\hline Boron & $5.12 \mathrm{E} 0$ & $1.37 \mathrm{E} 0$ & $"$ & $5.00 \mathrm{E} 0$ & $80-120$ \\
\hline Barium & $5.13 \mathrm{E} 0$ & $1.24 \mathrm{E}-1$ & $"$ & $5.00 \mathrm{E} 0$ & $80-120$ \\
\hline Beryllium & $5.14 \mathrm{E} 0$ & $1.31 \mathrm{E}-2$ & $"$ & $5.00 \mathrm{E} 0$ & $80-120$ \\
\hline Calcium & $5.12 \mathrm{E} 0$ & $4.52 \mathrm{E}-1$ & $"$ & $5.00 \mathrm{E} 0$ & $80-120$ \\
\hline Cobalt & $5.00 \mathrm{E} 0$ & $1.31 \mathrm{E}-1$ & $"$ & $5.00 \mathrm{E} 0$ & $80-120$ \\
\hline Copper & $5.00 \mathrm{E} 0$ & $8.78 \mathrm{E}-2$ & $"$ & $5.00 \mathrm{E} 0$ & $80-120$ \\
\hline Iron & $5.06 \mathrm{E} 0$ & $1.05 \mathrm{E}-1$ & $"$ & $5.00 \mathrm{E} 0$ & $80-120$ \\
\hline Potassium & $5.05 \mathrm{E} 1$ & $1.29 \mathrm{E} 0$ & $"$ & $5.00 \mathrm{E} 1$ & $80-120$ \\
\hline Magnesium & $4.88 \mathrm{E} 0$ & $4.89 \mathrm{E}-2$ & $"$ & $5.00 \mathrm{E} 0$ & $80-120$ \\
\hline Manganese & $5.10 \mathrm{E} 0$ & $6.25 \mathrm{E}-2$ & $"$ & $5.00 \mathrm{E} 0$ & $80-120$ \\
\hline Molybdenum & $5.08 \mathrm{E} 0$ & $1.41 \mathrm{E}-1$ & $"$ & $5.00 \mathrm{E} 0$ & $80-120$ \\
\hline Vanadium & $4.95 \mathrm{E} 0$ & $1.33 \mathrm{E}-1$ & $"$ & $5.00 \mathrm{E} 0$ & $80-120$ \\
\hline Zinc & 4.95E0 & $9.77 \mathrm{E}-2$ & $"$ & $5.00 \mathrm{E} 0$ & $80-120$ \\
\hline Sodium & 5.34E0 & 7.97E-1 & $"$ & $5.00 \mathrm{E} 0$ & $80-120$ \\
\hline
\end{tabular}


Total Metals by PNNL-AGG-ICP-AES/Water Extract - Quality Control

Environmental Science Laboratory

\begin{tabular}{|lrrrrrrrrrrr}
\hline & & Reporting & & Spike & Source & & \%REC & & RPD \\
Analyte & Result & Limit & Units & Level & Result & \%REC & Limits & RPD & Limit & Notes \\
\hline
\end{tabular}

Batch 9E12002 - 1:1 Water Extract (ICP/ICPMS)

Duplicate (9E12002-DUP1)

Aluminum

Boron

Barium

Beryllium

Bismuth

Calcium

Cobalt

Copper

Iron

Potassium

Lithium

Magnesium

Manganese

Molybdenum

Phosphorus

Strontium

Vanadium

Zinc

Sodium

Zirconium
Source: 0902002-01

$<1.01 \mathrm{E}-1 \quad 1.01 \mathrm{E}-1 \quad \mathrm{ug} / \mathrm{g}$ dry

$<1.48 \mathrm{E} 0$

$<1.34 \mathrm{E}-1$

$<1.41 \mathrm{E}-2$

$<3.28 \mathrm{E}-1$

$1.02 \mathrm{E} 1$

$<1.41 \mathrm{E}-1$

$<9.48 \mathrm{E}-2$

$<1.13 \mathrm{E}-1$

$4.15 \mathrm{E} 0$

$<8.06 \mathrm{E}-1$

2.19E0

$<6.74 \mathrm{E}-2$

$<1.52 \mathrm{E}-1$

$<6.51 \mathrm{E}-1$

$<8.65 \mathrm{E}-2$

$<1.44 \mathrm{E}-1$

$<1.05 \mathrm{E}-1$

1.20E 1

$<1.39 \mathrm{E}-1$

$\begin{array}{rr}1.01 \mathrm{E}-1 & \text { ug/g dry } \\ 1.48 \mathrm{E} 0 & " \\ 1.34 \mathrm{E}-1 & " \\ 1.41 \mathrm{E}-2 & " \\ 3.28 \mathrm{E}-1 & " \\ 4.88 \mathrm{E}-1 & " \\ 1.41 \mathrm{E}-1 & " \\ 9.48 \mathrm{E}-2 & " \\ 1.13 \mathrm{E}-1 & " \\ 1.39 \mathrm{E} 0 & " \\ 8.06 \mathrm{E}-1 & " \\ 5.27 \mathrm{E}-2 & " \\ 6.74 \mathrm{E}-2 & " \\ 1.52 \mathrm{E}-1 & " \\ 6.51 \mathrm{E}-1 & " \\ 8.65 \mathrm{E}-2 & " \\ 1.44 \mathrm{E}-1 & " \\ 1.05 \mathrm{E}-1 & " \\ 8.60 \mathrm{E}-1 & " \\ 1.39 \mathrm{E}-1 & "\end{array}$

Prepared: 03/09/09 Analyzed: 05/13/09

\begin{tabular}{|c|c|c|}
\hline $1.10 \mathrm{E}-1$ & & 35 \\
\hline ND & & 35 \\
\hline ND & & 35 \\
\hline ND & & 35 \\
\hline ND & & 35 \\
\hline $1.11 \mathrm{E} 1$ & 8.04 & 35 \\
\hline ND & & 35 \\
\hline ND & & 35 \\
\hline ND & & 35 \\
\hline $4.40 \mathrm{E} 0$ & 5.94 & 35 \\
\hline ND & & 35 \\
\hline $2.40 \mathrm{E} 0$ & 9.10 & 35 \\
\hline ND & & 35 \\
\hline ND & & 35 \\
\hline ND & & 35 \\
\hline ND & & 35 \\
\hline ND & & 3 \\
\hline ND & & 35 \\
\hline $1.26 \mathrm{E} 1$ & 5.54 & 35 \\
\hline ND & & 8 \\
\hline
\end{tabular}

Post Spike (9E12002-PS1)

Aluminum

Source: 0902002-01

Prepared: 05/12/09 Analyzed: 05/13/09

\begin{tabular}{|c|c|c|c|c|c|c|}
\hline $5.16 \mathrm{E} 2$ & N/A & $\mathrm{ug} / \mathrm{L}$ & $5.00 \mathrm{E} 2$ & $3.41 \mathrm{E} 1$ & 96.4 & $75-125$ \\
\hline $5.41 \mathrm{E} 2$ & N/A & $"$ & $5.00 \mathrm{E} 2$ & $1.55 \mathrm{E} 1$ & 105 & $75-125$ \\
\hline $2.67 \mathrm{E} 2$ & N/A & $"$ & $2.50 \mathrm{E} 2$ & $7.00 \mathrm{E} 0$ & 104 & $75-125$ \\
\hline $2.63 \mathrm{E} 2$ & N/A & $"$ & $2.50 \mathrm{E} 2$ & $1.23 \mathrm{E} 0$ & 105 & $75-125$ \\
\hline $4.66 \mathrm{E} 2$ & $\mathrm{~N} / \mathrm{A}$ & $"$ & $5.00 \mathrm{E} 2$ & ND & 96.1 & $75-125$ \\
\hline $3.99 \mathrm{E} 3$ & $\mathrm{~N} / \mathrm{A}$ & $"$ & $5.00 \mathrm{E} 2$ & $3.41 \mathrm{E} 3$ & 116 & $75-125$ \\
\hline $2.52 \mathrm{E} 2$ & N/A & $"$ & $2.50 \mathrm{E} 2$ & $3.57 \mathrm{E}-1$ & 101 & $75-125$ \\
\hline 5.19E2 & N/A & $"$ & $5.00 \mathrm{E} 2$ & $4.83 \mathrm{E} 0$ & 103 & $75-125$ \\
\hline $5.48 \mathrm{E} 2$ & $\mathrm{~N} / \mathrm{A}$ & $"$ & $5.00 \mathrm{E} 2$ & $3.20 \mathrm{E} 1$ & 103 & $75-125$ \\
\hline $2.68 \mathrm{E} 3$ & $\mathrm{~N} / \mathrm{A}$ & $"$ & $1.25 \mathrm{E} 3$ & $1.36 \mathrm{E} 3$ & 106 & $75-125$ \\
\hline $5.01 \mathrm{E} 2$ & $\mathrm{~N} / \mathrm{A}$ & $"$ & $5.00 \mathrm{E} 2$ & $4.15 \mathrm{E} 0$ & 99.5 & $75-125$ \\
\hline $1.25 \mathrm{E} 3$ & N/A & $"$ & $5.00 \mathrm{E} 2$ & 7.39E2 & 103 & $75-125$ \\
\hline $2.58 \mathrm{E} 2$ & $\mathrm{~N} / \mathrm{A}$ & $"$ & $2.50 \mathrm{E} 2$ & ND & 105 & $75-125$ \\
\hline $5.14 \mathrm{E} 2$ & $\mathrm{~N} / \mathrm{A}$ & $"$ & $5.00 \mathrm{E} 2$ & $9.74 \mathrm{E} 0$ & 101 & $75-125$ \\
\hline $1.29 \mathrm{E} 3$ & $\mathrm{~N} / \mathrm{A}$ & $"$ & $1.25 \mathrm{E} 3$ & $2.45 \mathrm{E} 1$ & 101 & $75-125$ \\
\hline $5.47 \mathrm{E} 2$ & N/A & $"$ & $5.00 \mathrm{E} 2$ & $1.81 \mathrm{E} 1$ & 106 & $75-125$ \\
\hline $2.53 \mathrm{E} 2$ & $\mathrm{~N} / \mathrm{A}$ & $"$ & $2.50 \mathrm{E} 2$ & $7.18 \mathrm{E} 0$ & 98.1 & $75-125$ \\
\hline $2.72 \mathrm{E} 2$ & N/A & $"$ & $2.50 \mathrm{E} 2$ & 1.64E1 & 102 & $75-125$ \\
\hline $4.53 \mathrm{E} 3$ & $\mathrm{~N} / \mathrm{A}$ & $"$ & $5.00 \mathrm{E} 2$ & $3.90 \mathrm{E} 3$ & 127 & $75-125$ \\
\hline $2.54 \mathrm{E} 2$ & N/A & $"$ & $2.50 \mathrm{E} 2$ & $4.08 \mathrm{E}-1$ & 101 & $75-125$ \\
\hline
\end{tabular}

$5.41 \mathrm{E} 2$

$2.67 \mathrm{E} 2$

Barium

Beryllium

$2.63 \mathrm{E} 2$

Bismuth

Calcium

Cobalt

Copper

Iron

Potassium

Lithium

Magnesium

Manganese

Molybdenum

Phosphorus

Strontium

Vanadium

Zinc

Sodium

Zirconium

$3.99 \mathrm{E} 3$

2.52E2

5.19E2

$5.48 \mathrm{E} 2$

$2.68 \mathrm{E} 3$

5.01E2

$1.25 \mathrm{E} 3$

$2.58 \mathrm{E} 2$

$5.14 \mathrm{E} 2$

$1.29 \mathrm{E} 3$

$5.47 \mathrm{E} 2$

$2.53 \mathrm{E} 2$

$2.72 \mathrm{E} 2$

$4.53 \mathrm{E} 3$

$2.54 \mathrm{E} 2$ 
Total Metals by PNNL-AGG-ICP-AES/Water Extract - Quality Control

Environmental Science Laboratory

\begin{tabular}{|lrrrrrrrrrrr}
\hline & & Reporting & & Spike & Source & & & $\%$ REC & & RPD \\
Analyte & Result & Limit & Units & Level & Result & \%REC & Limits & RPD & Limit & Notes \\
\hline
\end{tabular}

Batch 9E12003 - 1:1 Water Extract (ICP/ICPMS)

Blank (9E12003-BLK1)

Aluminum

Boron

Barium

Beryllium

Bismuth

Calcium

Cobalt

Copper

Iron

Potassium

Lithium

Magnesium

Manganese

Molybdenum

Phosphorus

Strontium

Vanadium

Zinc

Sodium

Zirconium
Prepared: 03/23/09 Analyzed: 05/13/09

$<9.37 \mathrm{E}-2 \quad 9.37 \mathrm{E}-2 \quad \mathrm{ug} / \mathrm{g}$ wet

$<1.37 \mathrm{E} 0 \quad 1.37 \mathrm{E} 0$

$<1.24 \mathrm{E}-1 \quad 1.24 \mathrm{E}-1$

$<1.31 \mathrm{E}-2 \quad 1.31 \mathrm{E}-2$

$<3.04 \mathrm{E}-1 \quad 3.04 \mathrm{E}-1$

$<4.52 \mathrm{E}-1 \quad 4.52 \mathrm{E}-1$

$<1.31 \mathrm{E}-1 \quad 1.31 \mathrm{E}-1$

$<8.78 \mathrm{E}-2 \quad 8.78 \mathrm{E}-2$

$<1.05 \mathrm{E}-1 \quad 1.05 \mathrm{E}-1$

$<1.29 \mathrm{E} 0 \quad 1.29 \mathrm{E} 0$

$<7.47 \mathrm{E}-1 \quad 7.47 \mathrm{E}-1$

$<4.89 \mathrm{E}-2 \quad 4.89 \mathrm{E}-2$

$<6.25 \mathrm{E}-2 \quad 6.25 \mathrm{E}-2$

$<1.41 \mathrm{E}-1 \quad 1.41 \mathrm{E}-1$

$<6.03 \mathrm{E}-1 \quad 6.03 \mathrm{E}-1$

$<8.02 \mathrm{E}-2 \quad 8.02 \mathrm{E}-2$

$<1.33 \mathrm{E}-1 \quad 1.33 \mathrm{E}-1$

$<9.77 \mathrm{E}-2 \quad 9.77 \mathrm{E}-2$

$<7.97 \mathrm{E}-1 \quad 7.97 \mathrm{E}-1$

$<1.29 \mathrm{E}-1 \quad 1.29 \mathrm{E}-1 \quad "$

LCS (9E12003-BS1)

Aluminum

Boron

Barium

Beryllium

Calcium

Cobalt

Copper

Iron

Potassium

Magnesium

Manganese

Molybdenum

Vanadium

Zinc

Sodium

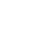

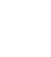


Total Metals by PNNL-AGG-ICP-AES/Water Extract - Quality Control

Environmental Science Laboratory

\begin{tabular}{|lrrrrrrrrrrr}
\hline & & Reporting & & Spike & Source & & \%REC & & RPD \\
Analyte & Result & Limit & Units & Level & Result & \%REC & Limits & RPD & Limit & Notes \\
\hline
\end{tabular}

Batch 9E12003 - 1:1 Water Extract (ICP/ICPMS)

\begin{tabular}{|c|c|c|c|c|c|c|c|c|c|}
\hline \multirow{2}{*}{$\begin{array}{l}\text { Duplicate (9E12003-DUP1) } \\
\text { Aluminum }\end{array}$} & \multicolumn{3}{|c|}{ Source: 0902002-03 } & \multicolumn{2}{|c|}{ Prepared: 03/23/09 } & \multicolumn{2}{|c|}{ Analyzed: 05/13/09 } & \multirow[b]{2}{*}{23.8} & \multirow[b]{2}{*}{35} \\
\hline & $4.65 \mathrm{E}-1$ & $1.04 \mathrm{E}-1$ & ug/g dry & & $5.90 \mathrm{E}-1$ & & & & \\
\hline Boron & $<1.52 \mathrm{E} 0$ & $1.52 \mathrm{E} 0$ & $"$ & & ND & & & & 35 \\
\hline Barium & $<1.37 \mathrm{E}-1$ & $1.37 \mathrm{E}-1$ & $"$ & & ND & & & & 35 \\
\hline Beryllium & $<1.45 \mathrm{E}-2$ & $1.45 \mathrm{E}-2$ & $"$ & & ND & & & & 35 \\
\hline Bismuth & $<3.37 \mathrm{E}-1$ & $3.37 \mathrm{E}-1$ & $"$ & & ND & & & & 35 \\
\hline Calcium & $2.33 \mathrm{E} 0$ & $5.01 \mathrm{E}-1$ & $"$ & & $2.39 \mathrm{E} 0$ & & & 2.34 & 35 \\
\hline Cobalt & $<1.45 \mathrm{E}-1$ & $1.45 \mathrm{E}-1$ & $"$ & & ND & & & & 35 \\
\hline Copper & $<9.73 \mathrm{E}-2$ & $9.73 \mathrm{E}-2$ & $"$ & & ND & & & & 35 \\
\hline Iron & $6.39 \mathrm{E}-1$ & $1.16 \mathrm{E}-1$ & $"$ & & $7.38 \mathrm{E}-1$ & & & 14.5 & 35 \\
\hline Potassium & $2.08 \mathrm{E} 0$ & $1.43 \mathrm{E} 0$ & $"$ & & $2.18 \mathrm{E} 0$ & & & 4.63 & 35 \\
\hline Lithium & $<8.28 \mathrm{E}-1$ & $8.28 \mathrm{E}-1$ & $"$ & & ND & & & & 35 \\
\hline Magnesium & $7.56 \mathrm{E}-1$ & $5.41 \mathrm{E}-2$ & $"$ & & $8.08 \mathrm{E}-1$ & & & 6.68 & 35 \\
\hline Manganese & $<6.92 \mathrm{E}-2$ & $6.92 \mathrm{E}-2$ & $"$ & & ND & & & & 35 \\
\hline Molybdenum & $<1.56 \mathrm{E}-1$ & $1.56 \mathrm{E}-1$ & $"$ & & ND & & & & 35 \\
\hline Phosphorus & $<6.68 \mathrm{E}-1$ & $6.68 \mathrm{E}-1$ & $"$ & & ND & & & & 35 \\
\hline Strontium & $<8.88 \mathrm{E}-2$ & $8.88 \mathrm{E}-2$ & $"$ & & ND & & & & 35 \\
\hline Vanadium & $<1.48 \mathrm{E}-1$ & $1.48 \mathrm{E}-1$ & $"$ & & ND & & & & 35 \\
\hline Zinc & $<1.08 \mathrm{E}-1$ & $1.08 \mathrm{E}-1$ & $"$ & & ND & & & & 35 \\
\hline Sodium & $1.15 \mathrm{E} 1$ & $8.83 \mathrm{E}-1$ & $"$ & & $1.13 \mathrm{E} 1$ & & & 1.38 & 35 \\
\hline Zirconium & $<1.42 \mathrm{E}-1$ & $1.42 \mathrm{E}-1$ & $"$ & & ND & & & & 35 \\
\hline Post Spike (9E12003-PS1) & \multicolumn{3}{|c|}{ Source: 0902002-03 } & \multicolumn{2}{|c|}{ Prepared: 05/12/09 } & \multicolumn{2}{|c|}{ Analyzed: 05/13/09 } & & \\
\hline Aluminum & $6.67 \mathrm{E} 2$ & N/A & $\mathrm{ug} / \mathrm{L}$ & $5.00 \mathrm{E} 2$ & $1.77 \mathrm{E} 2$ & 97.9 & $75-125$ & & \\
\hline Boron & $5.33 \mathrm{E} 2$ & N/A & $"$ & $5.00 \mathrm{E} 2$ & $6.76 \mathrm{E} 0$ & 105 & $75-125$ & & \\
\hline Barium & $2.68 \mathrm{E} 2$ & N/A & $"$ & $2.50 \mathrm{E} 2$ & $5.73 \mathrm{E} 0$ & 105 & $75-125$ & & \\
\hline Beryllium & $2.64 \mathrm{E} 2$ & N/A & $"$ & $2.50 \mathrm{E} 2$ & $3.29 \mathrm{E}-2$ & 105 & $75-125$ & & \\
\hline Bismuth & 4.81E2 & N/A & $"$ & $5.00 \mathrm{E} 2$ & ND & 97.9 & $75-125$ & & \\
\hline Calcium & $1.27 \mathrm{E} 3$ & N/A & $"$ & $5.00 \mathrm{E} 2$ & $7.18 \mathrm{E} 2$ & 110 & $75-125$ & & \\
\hline Cobalt & $2.50 \mathrm{E} 2$ & N/A & $"$ & $2.50 \mathrm{E} 2$ & ND & 101 & $75-125$ & & \\
\hline Copper & $5.19 \mathrm{E} 2$ & N/A & $"$ & $5.00 \mathrm{E} 2$ & $1.72 \mathrm{E} 0$ & 103 & $75-125$ & & \\
\hline Iron & $7.45 \mathrm{E} 2$ & N/A & $"$ & $5.00 \mathrm{E} 2$ & $2.22 \mathrm{E} 2$ & 105 & $75-125$ & & \\
\hline Potassium & $1.98 \mathrm{E} 3$ & N/A & $"$ & $1.25 \mathrm{E} 3$ & $6.56 \mathrm{E} 2$ & 106 & $75-125$ & & \\
\hline Lithium & $5.04 \mathrm{E} 2$ & N/A & $"$ & $5.00 \mathrm{E} 2$ & $4.70 \mathrm{E} 0$ & 99.8 & $75-125$ & & \\
\hline Magnesium & $7.50 \mathrm{E} 2$ & N/A & $"$ & $5.00 \mathrm{E} 2$ & $2.43 \mathrm{E} 2$ & 101 & $75-125$ & & \\
\hline Manganese & $2.66 \mathrm{E} 2$ & N/A & $"$ & $2.50 \mathrm{E} 2$ & $2.96 \mathrm{E}-1$ & 106 & $75-125$ & & \\
\hline Molybdenum & $5.08 \mathrm{E} 2$ & N/A & $"$ & $5.00 \mathrm{E} 2$ & ND & 102 & $75-125$ & & \\
\hline Phosphorus & $1.27 \mathrm{E} 3$ & N/A & $"$ & $1.25 \mathrm{E} 3$ & $3.22 \mathrm{E} 1$ & 99.3 & $75-125$ & & \\
\hline Strontium & $5.36 \mathrm{E} 2$ & N/A & $"$ & $5.00 \mathrm{E} 2$ & 4.73E0 & 106 & $75-125$ & & \\
\hline Vanadium & $2.60 \mathrm{E} 2$ & N/A & $"$ & $2.50 \mathrm{E} 2$ & $1.41 \mathrm{E} 1$ & 98.2 & $75-125$ & & \\
\hline Zinc & $2.76 \mathrm{E} 2$ & N/A & $"$ & $2.50 \mathrm{E} 2$ & $1.90 \mathrm{E} 1$ & 103 & $75-125$ & & \\
\hline Sodium & $4.09 \mathrm{E} 3$ & N/A & $"$ & $5.00 \mathrm{E} 2$ & $3.41 \mathrm{E} 3$ & 136 & $75-125$ & & \\
\hline Zirconium & $2.55 \mathrm{E} 2$ & N/A & $"$ & $2.50 \mathrm{E} 2$ & $5.64 \mathrm{E}-1$ & 102 & $75-125$ & & \\
\hline
\end{tabular}


Total Metals by PNNL-AGG-ICP-AES/Water Extract - Quality Control

Environmental Science Laboratory

\begin{tabular}{|lrrrrrrrrrrrr}
\hline & & Reporting & & Spike & Source & & \%REC & & RPD \\
Analyte & Result & Limit & Units & Level & Result & \%REC & Limits & RPD & Limit & Notes \\
\hline
\end{tabular}

Batch 9E12004 - 1:1 Water Extract (ICP/ICPMS)

Blank (9E12004-BLK1)

Aluminum

Boron

Barium

Beryllium

Bismuth

Calcium

Cobalt

Copper

Iron

Potassium

Lithium

Magnesium

Manganese

Molybdenum

Phosphorus

Strontium

Vanadium

Zinc

Sodium

Zirconium
Prepared: 04/03/09 Analyzed: 05/13/09

$<9.37 \mathrm{E}-2 \quad 9.37 \mathrm{E}-2 \quad \mathrm{ug} / \mathrm{g}$ wet

$<1.37 \mathrm{E} 0 \quad 1.37 \mathrm{E} 0$

$<1.24 \mathrm{E}-1 \quad 1.24 \mathrm{E}-1$

$<1.31 \mathrm{E}-2 \quad 1.31 \mathrm{E}-2$

$<3.04 \mathrm{E}-1 \quad 3.04 \mathrm{E}-1$

$<4.52 \mathrm{E}-1 \quad 4.52 \mathrm{E}-1$

$<1.31 \mathrm{E}-1 \quad 1.31 \mathrm{E}-1$

$<8.78 \mathrm{E}-2 \quad 8.78 \mathrm{E}-2$

$<1.05 \mathrm{E}-1 \quad 1.05 \mathrm{E}-1$

$<1.29 \mathrm{E} 0 \quad 1.29 \mathrm{E} 0$

$<7.47 \mathrm{E}-1 \quad 7.47 \mathrm{E}-1$

$<4.89 \mathrm{E}-2 \quad 4.89 \mathrm{E}-2$

$<6.25 \mathrm{E}-2 \quad 6.25 \mathrm{E}-2$

$<1.41 \mathrm{E}-1 \quad 1.41 \mathrm{E}-1$

$<6.03 \mathrm{E}-1 \quad 6.03 \mathrm{E}-1$

$<8.02 \mathrm{E}-2 \quad 8.02 \mathrm{E}-2$

$<1.33 \mathrm{E}-1 \quad 1.33 \mathrm{E}-1$

$<9.77 \mathrm{E}-2 \quad 9.77 \mathrm{E}-2$

$<7.97 \mathrm{E}-1 \quad 7.97 \mathrm{E}-1$

$<1.29 \mathrm{E}-1 \quad 1.29 \mathrm{E}-1 \quad "$

LCS (9E12004-BS1)

Aluminum

Boron

Barium

Beryllium

Calcium

Cobalt

Copper

Iron

Potassium

Magnesium

Manganese

Molybdenum

Vanadium

Zinc

Sodium

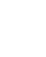

.


Total Metals by PNNL-AGG-ICP-AES/Water Extract - Quality Control

Environmental Science Laboratory

\begin{tabular}{|lrrrrrrrrrrr}
\hline & & Reporting & & Spike & Source & & \%REC & & RPD \\
Analyte & Result & Limit & Units & Level & Result & \%REC & Limits & RPD & Limit & Notes \\
\hline
\end{tabular}

Batch 9E12004 - 1:1 Water Extract (ICP/ICPMS)

\section{Duplicate (9E12004-DUP1)}

Aluminum

Boron

Barium

Beryllium

Bismuth

Calcium

Cobalt

Copper

Iron

Potassium

Lithium

Magnesium

Manganese

Molybdenum

Phosphorus

Strontium

Vanadium

Zinc

Sodium

Zirconium
Source: 0902002-08

$<9.52 \mathrm{E}-2 \quad 9.52 \mathrm{E}-2 \quad \mathrm{ug} / \mathrm{g}$ dry

$<1.39 \mathrm{E} 0$

$<1.26 \mathrm{E}-1$

$<1.33 \mathrm{E}-2$

$<3.09 \mathrm{E}-1$

$5.36 \mathrm{E} 1$

$<1.33 \mathrm{E}-1$

$<8.92 \mathrm{E}-2$

$<1.07 \mathrm{E}-1$

$6.34 \mathrm{E} 0$

$<7.59 \mathrm{E}-1$

$1.16 \mathrm{E} 1$

$<6.34 \mathrm{E}-2$

$<1.43 \mathrm{E}-1$

$1.47 \mathrm{E} 1$

2.49E-1

$<1.35 \mathrm{E}-1$

$<9.93 \mathrm{E}-2$

2.81E1

$<1.31 \mathrm{E}-1$

$\begin{array}{ll}1.39 \mathrm{E} 0 & " \\ 1.26 \mathrm{E}-1 & " \\ 1.33 \mathrm{E}-2 & " \\ 3.09 \mathrm{E}-1 & " \\ 4.59 \mathrm{E}-1 & " \\ 1.33 \mathrm{E}-1 & " \\ 8.92 \mathrm{E}-2 & " \\ 1.07 \mathrm{E}-1 & " \\ 1.31 \mathrm{E} 0 & " \\ 7.59 \mathrm{E}-1 & " \\ 4.96 \mathrm{E}-2 & " \\ 6.34 \mathrm{E}-2 & " \\ 1.43 \mathrm{E}-1 & " \\ 6.12 \mathrm{E}-1 & " \\ 8.14 \mathrm{E}-2 & " \\ 1.35 \mathrm{E}-1 & " \\ 9.93 \mathrm{E}-2 & " \\ 8.10 \mathrm{E}-1 & " \\ 1.31 \mathrm{E}-1 & "\end{array}$

Prepared: 04/03/09 Analyzed: 05/13/09

ND 35

ND

ND

ND

ND

$5.45 \mathrm{E} 1$

ND

ND

ND

6.38E0

ND

1.17E1

ND

ND

$1.47 \mathrm{E} 1$

2.54E-1

ND

ND

2.84E1

ND

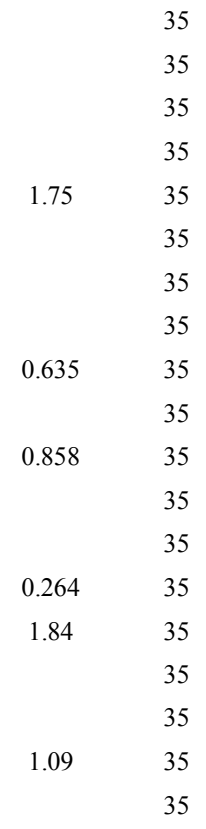

Post Spike (9E12004-PS1)

Source: 0902002-08 Prepared: 05/12/09 Analyzed: 05/13/09

\begin{tabular}{ll}
\hline Aluminum & $5.14 \mathrm{E} 2$ \\
Boron & $5.46 \mathrm{E} 2$ \\
Barium & $2.97 \mathrm{E} 2$ \\
Beryllium & $2.66 \mathrm{E} 2$ \\
Bismuth & $5.16 \mathrm{E} 2$ \\
Calcium & $1.87 \mathrm{E} 4$ \\
Cobalt & $2.51 \mathrm{E} 2$ \\
Copper & $5.30 \mathrm{E} 2$ \\
Iron & $5.32 \mathrm{E} 2$ \\
Potassium & $3.53 \mathrm{E} 3$ \\
Lithium & $5.14 \mathrm{E} 2$ \\
Magnesium & $4.51 \mathrm{E} 3$ \\
Manganese & $2.59 \mathrm{E} 2$ \\
Molybdenum & $5.34 \mathrm{E} 2$ \\
Phosphorus & $6.31 \mathrm{E} 3$ \\
Strontium & $6.29 \mathrm{E} 2$ \\
Vanadium & $2.50 \mathrm{E} 2$ \\
Zinc & $2.76 \mathrm{E} 2$ \\
Sodium & $1.01 \mathrm{E} 4$ \\
Zirconium & $2.70 \mathrm{E} 2$
\end{tabular}

\begin{tabular}{|c|c|c|c|c|c|}
\hline 2002 & & Prepared & $5 / 12 / 09$ & Analyz & $5 / 13 / 09$ \\
\hline N/A & $\mathrm{ug} / \mathrm{L}$ & $5.00 \mathrm{E} 2$ & $1.92 \mathrm{E} 1$ & 99 & $75-125$ \\
\hline N/A & $"$ & $5.00 \mathrm{E} 2$ & $5.71 \mathrm{E} 0$ & 108 & $75-125$ \\
\hline N/A & $"$ & $2.50 \mathrm{E} 2$ & $2.84 \mathrm{E} 1$ & 107 & $75-125$ \\
\hline N/A & $"$ & $2.50 \mathrm{E} 2$ & ND & 106 & $75-125$ \\
\hline N/A & $"$ & $5.00 \mathrm{E} 2$ & $1.33 \mathrm{E} 1$ & 101 & $75-125$ \\
\hline N/A & $"$ & $5.00 \mathrm{E} 2$ & $1.82 \mathrm{E} 4$ & 105 & $75-125$ \\
\hline N/A & $"$ & $2.50 \mathrm{E} 2$ & ND & 101 & $75-125$ \\
\hline N/A & $"$ & $5.00 \mathrm{E} 2$ & $1.61 \mathrm{E} 0$ & 106 & $75-125$ \\
\hline N/A & $"$ & $5.00 \mathrm{E} 2$ & 2.63E0 & 106 & $75-125$ \\
\hline N/A & $"$ & $1.25 \mathrm{E} 3$ & $2.13 \mathrm{E} 3$ & 112 & $75-125$ \\
\hline N/A & $"$ & $5.00 \mathrm{E} 2$ & $5.78 \mathrm{E} 0$ & 102 & $75-125$ \\
\hline N/A & $"$ & $5.00 \mathrm{E} 2$ & $3.90 \mathrm{E} 3$ & 123 & $75-125$ \\
\hline N/A & $"$ & $2.50 \mathrm{E} 2$ & ND & 106 & $75-125$ \\
\hline N/A & $"$ & $5.00 \mathrm{E} 2$ & $1.71 \mathrm{E} 1$ & 103 & $75-125$ \\
\hline N/A & $"$ & $1.25 \mathrm{E} 3$ & $4.89 \mathrm{E} 3$ & 114 & $75-125$ \\
\hline N/A & $"$ & $5.00 \mathrm{E} 2$ & $8.46 \mathrm{E} 1$ & 109 & $75-125$ \\
\hline N/A & $"$ & $2.50 \mathrm{E} 2$ & ND & 101 & $75-125$ \\
\hline N/A & $"$ & $2.50 \mathrm{E} 2$ & $2.02 \mathrm{E} 1$ & 102 & $75-125$ \\
\hline N/A & $"$ & $5.00 \mathrm{E} 2$ & $9.48 \mathrm{E} 3$ & 124 & $75-125$ \\
\hline N/A & $"$ & $2.50 \mathrm{E} 2$ & 2.07E0 & 107 & $75-125$ \\
\hline
\end{tabular}


Total Metals by PNNL-AGG-ICP-AES/Water Extract - Quality Control

Environmental Science Laboratory

\begin{tabular}{|lrrrrrrrrrrr}
\hline & & Reporting & & Spike & Source & & \%REC & & RPD \\
Analyte & Result & Limit & Units & Level & Result & \%REC & Limits & RPD & Limit & Notes \\
\hline
\end{tabular}

Batch 9E12005 - 1:1 Water Extract (ICP/ICPMS)

\begin{tabular}{|c|c|c|c|c|c|}
\hline Blank (9E12005-BLK1) & & & & Prepared: 04/20/09 & Analyzed: 05/13/09 \\
\hline Aluminum & $<9.37 \mathrm{E}-2$ & $9.37 \mathrm{E}-2$ & ug/g wet & & \\
\hline Boron & $<1.37 \mathrm{E} 0$ & $1.37 \mathrm{E} 0$ & $"$ & & \\
\hline Barium & $<1.24 \mathrm{E}-1$ & $1.24 \mathrm{E}-1$ & $"$ & & \\
\hline Beryllium & $<1.31 \mathrm{E}-2$ & $1.31 \mathrm{E}-2$ & $"$ & & \\
\hline Bismuth & $<3.04 \mathrm{E}-1$ & $3.04 \mathrm{E}-1$ & $"$ & & \\
\hline Calcium & $<4.52 \mathrm{E}-1$ & $4.52 \mathrm{E}-1$ & $"$ & & \\
\hline Cobalt & $<1.31 \mathrm{E}-1$ & $1.31 \mathrm{E}-1$ & $"$ & & \\
\hline Copper & $<8.78 \mathrm{E}-2$ & $8.78 \mathrm{E}-2$ & $"$ & & \\
\hline Iron & $<1.05 \mathrm{E}-1$ & $1.05 \mathrm{E}-1$ & $"$ & & \\
\hline Potassium & $<1.29 \mathrm{E} 0$ & $1.29 \mathrm{E} 0$ & $"$ & & \\
\hline Lithium & $<7.47 \mathrm{E}-1$ & $7.47 \mathrm{E}-1$ & $"$ & & \\
\hline Magnesium & $<4.89 \mathrm{E}-2$ & $4.89 \mathrm{E}-2$ & $"$ & & \\
\hline Manganese & $<6.25 \mathrm{E}-2$ & $6.25 \mathrm{E}-2$ & $"$ & & \\
\hline Molybdenum & $<1.41 \mathrm{E}-1$ & $1.41 \mathrm{E}-1$ & $"$ & & \\
\hline Phosphorus & $<6.03 \mathrm{E}-1$ & $6.03 \mathrm{E}-1$ & $"$ & & \\
\hline Strontium & $<8.02 \mathrm{E}-2$ & $8.02 \mathrm{E}-2$ & $"$ & & \\
\hline Vanadium & $<1.33 \mathrm{E}-1$ & $1.33 \mathrm{E}-1$ & $"$ & & \\
\hline Zinc & $1.30 \mathrm{E}-1$ & $9.77 \mathrm{E}-2$ & $"$ & & \\
\hline Sodium & $<7.97 \mathrm{E}-1$ & 7.97E-1 & $"$ & & \\
\hline Zirconium & $<1.29 \mathrm{E}-1$ & $1.29 \mathrm{E}-1$ & $"$ & & \\
\hline LCS (9E12005-BS1) & & & & Prepared: 04/20/09 & Analyzed: 05/13/09 \\
\hline Aluminum & 4.77E0 & $9.37 \mathrm{E}-2$ & ug/g wet & $5.00 \mathrm{E} 0$ & $80-120$ \\
\hline Boron & $5.16 \mathrm{E} 0$ & $1.37 \mathrm{E} 0$ & $"$ & $5.00 \mathrm{E} 0$ & $80-120$ \\
\hline Barium & $5.09 \mathrm{E} 0$ & $1.24 \mathrm{E}-1$ & $"$ & $5.00 \mathrm{E} 0$ & $80-120$ \\
\hline Beryllium & $5.10 \mathrm{E} 0$ & $1.31 \mathrm{E}-2$ & $"$ & $5.00 \mathrm{E} 0$ & $80-120$ \\
\hline Calcium & $5.04 \mathrm{E} 0$ & $4.52 \mathrm{E}-1$ & $"$ & $5.00 \mathrm{E} 0$ & $80-120$ \\
\hline Cobalt & $5.01 \mathrm{E} 0$ & $1.31 \mathrm{E}-1$ & $"$ & $5.00 \mathrm{E} 0$ & $80-120$ \\
\hline Copper & $4.98 \mathrm{E} 0$ & $8.78 \mathrm{E}-2$ & $"$ & $5.00 \mathrm{E} 0$ & $80-120$ \\
\hline Iron & $5.00 \mathrm{E} 0$ & $1.05 \mathrm{E}-1$ & $"$ & $5.00 \mathrm{E} 0$ & $80-120$ \\
\hline Potassium & $4.99 \mathrm{E} 1$ & $1.29 \mathrm{E} 0$ & $"$ & $5.00 \mathrm{E} 1$ & $80-120$ \\
\hline Magnesium & $4.83 \mathrm{E} 0$ & $4.89 \mathrm{E}-2$ & $"$ & $5.00 \mathrm{E} 0$ & $80-120$ \\
\hline Manganese & $5.05 \mathrm{E} 0$ & $6.25 \mathrm{E}-2$ & $"$ & $5.00 \mathrm{E} 0$ & $80-120$ \\
\hline Molybdenum & $5.10 \mathrm{E} 0$ & $1.41 \mathrm{E}-1$ & $"$ & $5.00 \mathrm{E} 0$ & $80-120$ \\
\hline Vanadium & $4.91 \mathrm{E} 0$ & $1.33 \mathrm{E}-1$ & $"$ & $5.00 \mathrm{E} 0$ & $80-120$ \\
\hline Zinc & $4.95 \mathrm{E} 0$ & $9.77 \mathrm{E}-2$ & $"$ & $5.00 \mathrm{E} 0$ & $80-120$ \\
\hline Sodium & $5.00 \mathrm{E} 0$ & 7.97E-1 & $"$ & $5.00 \mathrm{E} 0$ & $80-120$ \\
\hline
\end{tabular}


Total Metals by PNNL-AGG-ICP-AES/Water Extract - Quality Control

Environmental Science Laboratory

\begin{tabular}{|lrrrrrrrrrrr}
\hline & & Reporting & & Spike & Source & & & $\%$ REC & & RPD \\
Analyte & Result & Limit & Units & Level & Result & $\%$ REC & Limits & RPD & Limit & Notes \\
\hline
\end{tabular}

Batch 9E12005 - 1:1 Water Extract (ICP/ICPMS)

Duplicate (9E12005-DUP1)

Aluminum

Boron

Barium

Beryllium

Bismuth

Calcium

Cobalt

Copper

Iron

Potassium

Lithium

Magnesium

Manganese

Molybdenum

Phosphorus

Strontium

Vanadium

Zinc

Sodium

Zirconium

Sou

Source: 0902002-12

3.39E-1 9.45E-2 ug/g dry

$<1.38 \mathrm{E} 0$

$<1.25 \mathrm{E}-1$

$<1.32 \mathrm{E}-2$

$<3.07 \mathrm{E}-1$

$2.01 \mathrm{E} 1$

$<1.32 \mathrm{E}-1$

$<8.86 \mathrm{E}-2$

$1.26 \mathrm{E}-1$

$5.71 \mathrm{E} 0$

$<7.53 \mathrm{E}-1$

$6.22 \mathrm{E} 0$

$<6.30 \mathrm{E}-2$

$<1.42 \mathrm{E}-1$

$<6.08 \mathrm{E}-1$

$9.34 \mathrm{E}-2$

$<1.34 \mathrm{E}-1$

$<9.86 \mathrm{E}-2$

1.61E 1

$<1.30 \mathrm{E}-1$

$\begin{array}{cc}9.45 \mathrm{E}-2 & \text { ug/g dry } \\ 1.38 \mathrm{E} 0 & " \\ 1.25 \mathrm{E}-1 & " \\ 1.32 \mathrm{E}-2 & " \\ 3.07 \mathrm{E}-1 & " \\ 4.56 \mathrm{E}-1 & " \\ 1.32 \mathrm{E}-1 & " \\ 8.86 \mathrm{E}-2 & " \\ 1.06 \mathrm{E}-1 & " \\ 1.30 \mathrm{E} 0 & " \\ 7.53 \mathrm{E}-1 & " \\ 4.93 \mathrm{E}-2 & " \\ 6.30 \mathrm{E}-2 & " \\ 1.42 \mathrm{E}-1 & " \\ 6.08 \mathrm{E}-1 & " \\ 8.08 \mathrm{E}-2 & " \\ 1.34 \mathrm{E}-1 & " \\ 9.86 \mathrm{E}-2 & " \\ 8.04 \mathrm{E}-1 & " \\ 1.30 \mathrm{E}-1 & "\end{array}$

Prepared: 04/20/09 Analyzed: 05/13/09

\begin{tabular}{|c|c|c|}
\hline $3.10 \mathrm{E}-1$ & 9.17 & 35 \\
\hline ND & & 35 \\
\hline ND & & 35 \\
\hline ND & & 35 \\
\hline ND & & 35 \\
\hline $1.98 \mathrm{E} 1$ & 1.69 & 35 \\
\hline ND & & 35 \\
\hline ND & & 35 \\
\hline ND & & 35 \\
\hline 5.99E0 & 4.85 & 35 \\
\hline ND & & 35 \\
\hline 5.99E0 & 3.75 & 35 \\
\hline ND & & 35 \\
\hline ND & & 35 \\
\hline ND & & 35 \\
\hline $9.00 \mathrm{E}-2$ & 3.67 & 35 \\
\hline ND & & 35 \\
\hline ND & & 35 \\
\hline $1.67 \mathrm{E} 1$ & 3.56 & 35 \\
\hline ND & & 35 \\
\hline
\end{tabular}

Post Spike (9E12005-PS1)

Aluminum

Source: 0902002-12

Prepared: 05/12/09 Analyzed: 05/13/09

Boron

5.97E2

$5.70 \mathrm{E} 2$

Barium

$2.81 \mathrm{E} 2$

Beryllium

$2.70 \mathrm{E} 2$

Bismuth

Calcium

Cobalt

Copper

Iron

Potassium

Lithium

Magnesium

Manganese

Molybdenum

Phosphorus

Strontium

Vanadium

Zinc

Sodium

Zirconium

$5.14 \mathrm{E} 2$

$7.16 \mathrm{E} 3$

$2.56 \mathrm{E} 2$

$5.35 \mathrm{E} 2$

$5.60 \mathrm{E} 2$

$3.35 \mathrm{E} 3$

5.14E2

$2.56 \mathrm{E} 3$

2.62E2

5.37E2

$1.36 \mathrm{E} 3$

$5.68 \mathrm{E} 2$

$2.59 \mathrm{E} 2$

$2.86 \mathrm{E} 2$

$6.19 \mathrm{E} 3$

$2.59 \mathrm{E} 2$

0902002-12

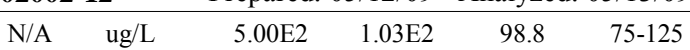

$\begin{array}{llllll}\mathrm{N} / \mathrm{A} & \quad & 5.00 \mathrm{E} 2 & 6.70 \mathrm{E} 0 & 113 & 75-125\end{array}$

$\begin{array}{lllll}\mathrm{N} / \mathrm{A} & \quad 2.50 \mathrm{E} 2 & 1.52 \mathrm{E} 1 & 106 & 75-125\end{array}$

$\begin{array}{lllll}\mathrm{N} / \mathrm{A} & \quad 2.50 \mathrm{E} 2 & \mathrm{ND} & 108 & 75-125\end{array}$

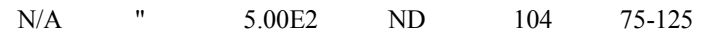

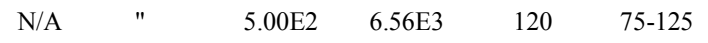

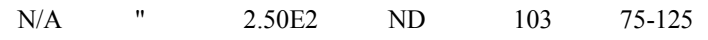

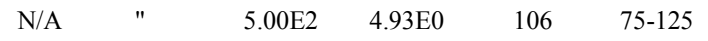

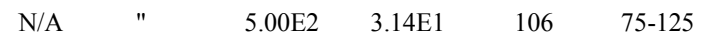

$\begin{array}{llllll}\mathrm{N} / \mathrm{A} & \quad 1.25 \mathrm{E} 3 & 1.98 \mathrm{E} 3 & 109 & 75-125\end{array}$

$\begin{array}{llllll}\mathrm{N} / \mathrm{A} & \quad & 5.00 \mathrm{E} 2 & 4.48 \mathrm{E} 0 & 102 & 75-125\end{array}$

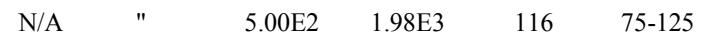

$\begin{array}{llllll}\text { N/A } & \text { " } & 2.50 \mathrm{E} 2 & \mathrm{ND} & 107 & 75-125\end{array}$

$\begin{array}{llllll}\mathrm{N} / \mathrm{A} & \quad & 5.00 \mathrm{E} 2 & 1.57 \mathrm{E} 1 & 104 & 75-125\end{array}$

$\begin{array}{llllll}\mathrm{N} / \mathrm{A} & \quad & 1.25 \mathrm{E} 3 & 6.32 \mathrm{E} 1 & 104 & 75-125\end{array}$

$\begin{array}{llllll}\mathrm{N} / \mathrm{A} & \quad & 5.00 \mathrm{E} 2 & 2.98 \mathrm{E} 1 & 108 & 75-125\end{array}$

$\begin{array}{llllll}\mathrm{N} / \mathrm{A} & \quad & 2.50 \mathrm{E} 2 & 6.68 \mathrm{E} 0 & 101 & 75-125\end{array}$

$\begin{array}{llllll}\mathrm{N} / \mathrm{A} & \quad & 2.50 \mathrm{E} 2 & 1.83 \mathrm{E} 1 & 107 & 75-125\end{array}$

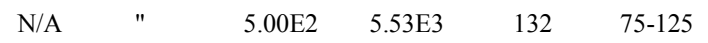

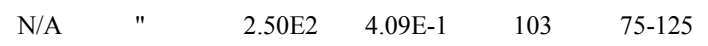


Total Metals by PNNL-AGG-ICP-AES/Acid Extract - Quality Control

Environmental Science Laboratory

\begin{tabular}{|lrrrrrrrrrrr}
\hline & & Reporting & & Spike & Source & & \%REC & & RPD \\
Analyte & Result & Limit & Units & Level & Result & $\%$ REC & Limits & RPD & Limit & Notes \\
\hline
\end{tabular}

Batch 9E12001 - ASTM D 5198 (ICP/ICPMS)

\begin{tabular}{|c|c|c|c|c|c|}
\hline Blank (9E12001-BLK1) & & & & Prepared: 04/24/09 & Analyzed: 05/12/09 \\
\hline Aluminum & $<6.76 \mathrm{E} 0$ & $6.76 \mathrm{E} 0$ & ug/g wet & & \\
\hline Boron & $<2.90 \mathrm{E} 1$ & $2.90 \mathrm{E} 1$ & $"$ & & \\
\hline Barium & $<4.12 \mathrm{E}-1$ & $4.12 \mathrm{E}-1$ & $"$ & & \\
\hline Beryllium & $<2.31 \mathrm{E}-1$ & $2.31 \mathrm{E}-1$ & $"$ & & \\
\hline Bismuth & $<2.85 \mathrm{E} 0$ & $2.85 \mathrm{E} 0$ & $"$ & & \\
\hline Calcium & $<7.02 \mathrm{E} 0$ & 7.02E0 & $"$ & & \\
\hline Cobalt & $<1.44 \mathrm{E} 0$ & $1.44 \mathrm{E} 0$ & $"$ & & \\
\hline Copper & $<1.93 \mathrm{E} 0$ & $1.93 \mathrm{E} 0$ & $"$ & & \\
\hline Iron & $<1.82 \mathrm{E} 0$ & $1.82 \mathrm{E} 0$ & $"$ & & \\
\hline Potassium & $<2.59 \mathrm{E} 1$ & $2.59 \mathrm{E} 1$ & $"$ & & \\
\hline Lithium & $<6.28 \mathrm{E} 0$ & $6.28 \mathrm{E} 0$ & $"$ & & \\
\hline Magnesium & $<1.92 \mathrm{E} 0$ & $1.92 \mathrm{E} 0$ & $"$ & & \\
\hline Manganese & $<6.04 \mathrm{E}-1$ & $6.04 \mathrm{E}-1$ & $"$ & & \\
\hline Molybdenum & $<2.29 \mathrm{E} 0$ & $2.29 \mathrm{E} 0$ & $"$ & & \\
\hline Phosphorus & $<8.93 \mathrm{E} 0$ & 8.93E0 & $"$ & & \\
\hline Strontium & $<4.40 \mathrm{E}-1$ & $4.40 \mathrm{E}-1$ & $"$ & & \\
\hline Vanadium & $<1.21 \mathrm{E} 0$ & $1.21 \mathrm{E} 0$ & $"$ & & \\
\hline Zinc & $<1.10 \mathrm{E} 0$ & $1.10 \mathrm{E} 0$ & $"$ & & \\
\hline Sodium & $<1.90 \mathrm{E} 1$ & $1.90 \mathrm{E} 1$ & $"$ & & \\
\hline Zirconium & $<8.10 \mathrm{E}-1$ & $8.10 \mathrm{E}-1$ & $"$ & & \\
\hline LCS (9E12001-BS1) & & & & Prepared: 04/24/09 & Analyzed: 05/12/09 \\
\hline Aluminum & $5.60 \mathrm{E} 0$ & $6.76 \mathrm{E}-1$ & ug/g wet & $5.94 \mathrm{E} 0$ & $80-120$ \\
\hline Boron & $5.72 \mathrm{E} 0$ & $2.90 \mathrm{E} 0$ & $"$ & 5.94E0 & $80-120$ \\
\hline Barium & $5.81 \mathrm{E} 0$ & $4.12 \mathrm{E}-2$ & $"$ & $5.94 \mathrm{E} 0$ & $80-120$ \\
\hline Beryllium & $5.85 \mathrm{E} 0$ & $2.31 \mathrm{E}-2$ & $"$ & 5.94E0 & $80-120$ \\
\hline Calcium & $6.04 \mathrm{E} 0$ & $7.02 \mathrm{E}-1$ & $"$ & $5.94 \mathrm{E} 0$ & $80-120$ \\
\hline Cobalt & $5.99 \mathrm{E} 0$ & $1.44 \mathrm{E}-1$ & $"$ & $5.94 \mathrm{E} 0$ & $80-120$ \\
\hline Copper & $5.96 \mathrm{E} 0$ & $1.93 \mathrm{E}-1$ & $"$ & $5.94 \mathrm{E} 0$ & $80-120$ \\
\hline Iron & $6.11 \mathrm{E} 0$ & $1.82 \mathrm{E}-1$ & $"$ & $5.94 \mathrm{E} 0$ & $80-120$ \\
\hline Potassium & $5.85 \mathrm{E} 1$ & $2.59 \mathrm{E} 0$ & $"$ & $5.94 \mathrm{E} 1$ & $80-120$ \\
\hline Magnesium & $5.50 \mathrm{E} 0$ & $1.92 \mathrm{E}-1$ & $"$ & 5.94E0 & $80-120$ \\
\hline Manganese & $5.95 \mathrm{E} 0$ & $6.04 \mathrm{E}-2$ & $"$ & $5.94 \mathrm{E} 0$ & $80-120$ \\
\hline Molybdenum & $6.03 \mathrm{E} 0$ & $2.29 \mathrm{E}-1$ & $"$ & $5.94 \mathrm{E} 0$ & $80-120$ \\
\hline Vanadium & $5.86 \mathrm{E} 0$ & $1.21 \mathrm{E}-1$ & $"$ & $5.94 \mathrm{E} 0$ & $80-120$ \\
\hline Zinc & $5.69 \mathrm{E} 0$ & $1.10 \mathrm{E}-1$ & $"$ & $5.94 \mathrm{E} 0$ & $80-120$ \\
\hline Sodium & $6.59 \mathrm{E} 0$ & $1.90 \mathrm{E} 0$ & $"$ & $5.94 \mathrm{E} 0$ & $80-120$ \\
\hline
\end{tabular}


Total Metals by PNNL-AGG-ICP-AES/Acid Extract - Quality Control

Environmental Science Laboratory

\begin{tabular}{|lrrrrrrrrrrr}
\hline & & Reporting & & Spike & Source & & \%REC & & RPD \\
Analyte & Result & Limit & Units & Level & Result & \%REC & Limits & RPD & Limit & Notes \\
\hline
\end{tabular}

Batch 9E12001 - ASTM D 5198 (ICP/ICPMS)

\begin{tabular}{|c|c|c|c|c|c|c|c|c|c|}
\hline \multirow{2}{*}{$\begin{array}{l}\text { Duplicate (9E12001-DUP1) } \\
\text { Aluminum }\end{array}$} & \multicolumn{3}{|c|}{ Source: 0902002-08 } & \multicolumn{2}{|c|}{ Prepared: 04/24/09 } & \multicolumn{2}{|c|}{ Analyzed: 05/12/09 } & \multirow[b]{2}{*}{2.21} & \multirow[b]{2}{*}{35} \\
\hline & $1.24 \mathrm{E} 4$ & $2.62 \mathrm{E} 1$ & ug/g dry & & $1.22 \mathrm{E} 4$ & & & & \\
\hline Boron & $<1.12 \mathrm{E} 2$ & $1.12 \mathrm{E} 2$ & $"$ & & ND & & & & 35 \\
\hline Barium & $1.10 \mathrm{E} 2$ & $1.60 \mathrm{E} 0$ & $"$ & & $1.07 \mathrm{E} 2$ & & & 2.66 & 35 \\
\hline Beryllium & $<8.95 \mathrm{E}-1$ & $8.95 \mathrm{E}-1$ & $"$ & & ND & & & & 35 \\
\hline Bismuth & $1.13 \mathrm{E} 1$ & $1.10 \mathrm{E} 1$ & $"$ & & $1.14 \mathrm{E} 1$ & & & 0.333 & 35 \\
\hline Calcium & $1.17 \mathrm{E} 4$ & $2.72 \mathrm{E} 1$ & $"$ & & $1.15 \mathrm{E} 4$ & & & 1.99 & 35 \\
\hline Cobalt & $9.13 \mathrm{E} 0$ & $5.60 \mathrm{E} 0$ & $"$ & & $9.12 \mathrm{E} 0$ & & & 0.0887 & 35 \\
\hline Copper & $1.43 \mathrm{E} 1$ & $7.49 \mathrm{E} 0$ & $"$ & & $1.43 \mathrm{E} 1$ & & & 0.0755 & 35 \\
\hline Iron & $1.96 \mathrm{E} 4$ & 7.04E0 & $"$ & & $1.94 \mathrm{E} 4$ & & & 0.723 & 35 \\
\hline Potassium & $2.24 \mathrm{E} 3$ & $1.00 \mathrm{E} 2$ & $"$ & & $2.23 \mathrm{E} 3$ & & & 0.286 & 35 \\
\hline Lithium & $<2.43 \mathrm{E} 1$ & $2.43 \mathrm{E} 1$ & $"$ & & ND & & & & 35 \\
\hline Magnesium & $7.28 \mathrm{E} 3$ & $7.46 \mathrm{E} 0$ & $"$ & & $7.24 \mathrm{E} 3$ & & & 0.546 & 35 \\
\hline Manganese & $3.94 \mathrm{E} 2$ & $2.34 \mathrm{E} 0$ & $"$ & & $3.98 \mathrm{E} 2$ & & & 0.994 & 35 \\
\hline Molybdenum & $<8.89 \mathrm{E} 0$ & 8.89E0 & $"$ & & ND & & & & 35 \\
\hline Phosphorus & $6.39 \mathrm{E} 2$ & $3.46 \mathrm{E} 1$ & $"$ & & $6.44 \mathrm{E} 2$ & & & 0.757 & 35 \\
\hline Strontium & $3.70 \mathrm{E} 1$ & $1.71 \mathrm{E} 0$ & $"$ & & $3.62 \mathrm{E} 1$ & & & 2.16 & 35 \\
\hline Vanadium & $2.06 \mathrm{E} 1$ & 4.69E0 & $"$ & & $2.10 \mathrm{E} 1$ & & & 1.69 & 35 \\
\hline Zinc & $4.66 \mathrm{E} 1$ & 4.27E0 & $"$ & & $4.60 \mathrm{E} 1$ & & & 1.22 & 35 \\
\hline Sodium & $2.26 \mathrm{E} 2$ & 7.38E1 & $"$ & & $2.21 \mathrm{E} 2$ & & & 1.92 & 35 \\
\hline Zirconium & 7.99E0 & $3.14 \mathrm{E} 0$ & $"$ & & $8.13 \mathrm{E} 0$ & & & 1.72 & 35 \\
\hline Post Spike (9E12001-PS1) & \multicolumn{3}{|c|}{ Source: 0902002-08 } & \multicolumn{4}{|c|}{ Prepared \& Analyzed: 05/12/09 } & & \\
\hline Aluminum & $6.41 \mathrm{E} 4$ & $\mathrm{~N} / \mathrm{A}$ & $\mathrm{ug} / \mathrm{L}$ & $5.00 \mathrm{E} 2$ & $6.21 \mathrm{E} 4$ & 407 & $75-125$ & & \\
\hline Boron & $5.09 \mathrm{E} 2$ & N/A & $"$ & $5.00 \mathrm{E} 2$ & ND & 104 & $75-125$ & & \\
\hline Barium & $8.08 \mathrm{E} 2$ & N/A & $"$ & $2.50 \mathrm{E} 2$ & $5.48 \mathrm{E} 2$ & 104 & $75-125$ & & \\
\hline Beryllium & $2.58 \mathrm{E} 2$ & $\mathrm{~N} / \mathrm{A}$ & $"$ & $2.50 \mathrm{E} 2$ & $1.82 \mathrm{E} 0$ & 102 & $75-125$ & & \\
\hline Bismuth & $5.77 \mathrm{E} 2$ & $\mathrm{~N} / \mathrm{A}$ & $"$ & $5.00 \mathrm{E} 2$ & $5.80 \mathrm{E} 1$ & 104 & $75-125$ & & \\
\hline Calcium & $6.05 \mathrm{E} 4$ & N/A & $"$ & $5.00 \mathrm{E} 2$ & $5.86 \mathrm{E} 4$ & 386 & $75-125$ & & \\
\hline Cobalt & $2.92 \mathrm{E} 2$ & N/A & $"$ & $2.50 \mathrm{E} 2$ & $4.66 \mathrm{E} 1$ & 98.1 & $75-125$ & & \\
\hline Copper & $5.89 \mathrm{E} 2$ & N/A & $"$ & $5.00 \mathrm{E} 2$ & $7.30 \mathrm{E} 1$ & 103 & $75-125$ & & \\
\hline Iron & $1.02 \mathrm{E} 5$ & N/A & $"$ & $5.00 \mathrm{E} 2$ & $9.92 \mathrm{E} 4$ & 538 & $75-125$ & & \\
\hline Potassium & $1.30 \mathrm{E} 4$ & N/A & $"$ & $1.25 \mathrm{E} 3$ & $1.14 \mathrm{E} 4$ & 125 & $75-125$ & & \\
\hline Lithium & $5.78 \mathrm{E} 2$ & N/A & $"$ & $5.00 \mathrm{E} 2$ & $8.02 \mathrm{E} 1$ & 99.5 & $75-125$ & & \\
\hline Magnesium & $3.75 \mathrm{E} 4$ & N/A & $"$ & $5.00 \mathrm{E} 2$ & $3.69 \mathrm{E} 4$ & 105 & $75-125$ & & \\
\hline Manganese & $2.35 \mathrm{E} 3$ & N/A & $"$ & $2.50 \mathrm{E} 2$ & $2.03 \mathrm{E} 3$ & 126 & $75-125$ & & \\
\hline Molybdenum & $5.40 \mathrm{E} 2$ & N/A & $"$ & $5.00 \mathrm{E} 2$ & $3.56 \mathrm{E} 1$ & 101 & $75-125$ & & \\
\hline Phosphorus & $4.65 \mathrm{E} 3$ & N/A & $"$ & $1.25 \mathrm{E} 3$ & $3.29 \mathrm{E} 3$ & 109 & $75-125$ & & \\
\hline Strontium & $6.99 \mathrm{E} 2$ & $\mathrm{~N} / \mathrm{A}$ & $"$ & $5.00 \mathrm{E} 2$ & $1.85 \mathrm{E} 2$ & 103 & $75-125$ & & \\
\hline Vanadium & $3.59 \mathrm{E} 2$ & N/A & $"$ & $2.50 \mathrm{E} 2$ & $1.07 \mathrm{E} 2$ & 101 & $75-125$ & & \\
\hline Zinc & 4.81E2 & N/A & $"$ & $2.50 \mathrm{E} 2$ & $2.35 \mathrm{E} 2$ & 98.3 & $75-125$ & & \\
\hline Sodium & $1.68 \mathrm{E} 3$ & N/A & $"$ & $5.00 \mathrm{E} 2$ & $1.13 \mathrm{E} 3$ & 111 & $75-125$ & & \\
\hline Zirconium & 2.91E2 & N/A & $"$ & $2.50 \mathrm{E} 2$ & $4.15 \mathrm{E} 1$ & 99.8 & $75-125$ & & \\
\hline
\end{tabular}


RCRA Metals By PNNL-AGG-415/Water Extract - Quality Control

Environmental Science Laboratory

\begin{tabular}{|lrrrrrrrrrrrr}
\hline & & Reporting & & Spike & Source & & & $\%$ REC & & RPD \\
Analyte & Result & Limit & Units & Level & Result & $\%$ REC & Limits & RPD & Limit & Notes \\
\hline
\end{tabular}

Batch 9E07001 - 1:1 Water Extract (ICP/ICPMS)

Blank (9E07001-BLK1)

Chromium

Arsenic

Selenium

Silver

Cadmium

Antimony

Lead

LCS (9E07001-BS1)

Chromium

Arsenic

Selenium

Silver

Cadmium

Lead

Duplicate (9E07001-DUP1)

Chromium

Arsenic

Selenium

Silver

Cadmium

Antimony

Lead

Post Spike (9E07001-PS1)

Chromium

Arsenic

Selenium

Silver

Cadmium

Antimony

Lead
Prepared \& Analyzed: 05/07/09

$\begin{array}{lll}<3.44 \mathrm{E}-3 & 3.44 \mathrm{E}-3 & \text { ug } / \mathrm{g} \text { wet } \\ <2.84 \mathrm{E}-3 & 2.84 \mathrm{E}-3 & " \\ <7.92 \mathrm{E}-3 & 7.92 \mathrm{E}-3 & " \\ <3.13 \mathrm{E}-3 & 3.13 \mathrm{E}-3 & " \\ <5.73 \mathrm{E}-4 & 5.73 \mathrm{E}-4 & " \\ <6.58 \mathrm{E}-4 & 6.58 \mathrm{E}-4 & " \\ <1.16 \mathrm{E}-3 & 1.16 \mathrm{E}-3\end{array}$

Prepared \& Analyzed: 05/07/09

$\begin{array}{llllll}5.16 \mathrm{E} 0 & 3.44 \mathrm{E}-1 & \mathrm{ug} / \mathrm{g} \text { wet } & 5.00 \mathrm{E} 0 & 103 & 80-120\end{array}$

$\begin{array}{llllll}5.42 \mathrm{E} 0 & 2.84 \mathrm{E}-1 & " & 5.00 \mathrm{E} 0 & 108 & 80-120\end{array}$

$\begin{array}{lllll}5.06 \mathrm{E} 0 & 7.92 \mathrm{E}-1 & & & \end{array}$

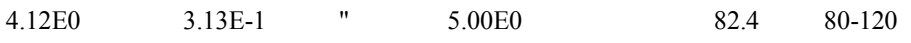

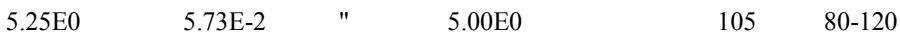

$\begin{array}{lllll}4.75 \mathrm{E} 0 & 1.16 \mathrm{E}-1 \quad & & & \end{array}$

Source: 0902002-01 Prepared \& Analyzed: 05/07/09

$\begin{array}{rccccc}<3.72 \mathrm{E}-3 & 3.72 \mathrm{E}-3 & \text { ug/g dry } & \text { ND } & 7.03 & 35 \\ 1.41 \mathrm{E}-2 & 3.06 \mathrm{E}-3 & " & 1.32 \mathrm{E}-2 & & 35 \\ <8.54 \mathrm{E}-3 & 8.54 \mathrm{E}-3 & " & \text { ND } & \text { ND } & 35 \\ <3.37 \mathrm{E}-3 & 3.37 \mathrm{E}-3 & " & \mathrm{ND} & 35 \\ <6.18 \mathrm{E}-4 & 6.18 \mathrm{E}-4 & " & \mathrm{ND} & 35 \\ <7.11 \mathrm{E}-4 & 7.11 \mathrm{E}-4 & " & \text { ND } & & 35\end{array}$

Source: 0902002-01 Prepared \& Analyzed: 05/07/09

$\begin{array}{lcccccc}4.80 \mathrm{E} 0 & \text { N/A } & \text { ug/L } & 5.00 \mathrm{E} 0 & 1.49 \mathrm{E}-3 & 95.9 & 75-125 \\ 7.73 \mathrm{E} 0 & \text { N/A } & " & 5.00 \mathrm{E} 0 & 2.44 \mathrm{E} 0 & 106 & 75-125 \\ 5.35 \mathrm{E} 0 & \text { N/A } & " & 5.00 \mathrm{E} 0 & 1.76 \mathrm{E}-1 & 103 & 75-125 \\ 5.02 \mathrm{E} 0 & \text { N/A } & " & 5.00 \mathrm{E} 0 & \text { ND } & 101 & 75-125 \\ 5.22 \mathrm{E} 0 & \text { N/A } & " & 5.00 \mathrm{E} 0 & \text { ND } & 107 & 75-125 \\ 5.38 \mathrm{E} 0 & \text { N/A } & " & 5.00 \mathrm{E} 0 & 3.18 \mathrm{E}-2 & 107 & 75-125 \\ 5.02 \mathrm{E} 0 & \text { N/A } & " & 5.00 \mathrm{E} 0 & 6.30 \mathrm{E}-2 & 99.1 & 75-125\end{array}$


RCRA Metals By PNNL-AGG-415/Water Extract - Quality Control

Environmental Science Laboratory

\begin{tabular}{|lrrrrrrrrrrrr}
\hline & & Reporting & & Spike & Source & & & $\%$ REC & & RPD \\
Analyte & Result & Limit & Units & Level & Result & $\%$ REC & Limits & RPD & Limit & Notes \\
\hline
\end{tabular}

Batch 9E07002 - 1:1 Water Extract (ICP/ICPMS)

Blank (9E07002-BLK1)

Chromium

Arsenic

Selenium

Silver

Cadmium

Antimony

Lead

LCS (9E07002-BS1)

Chromium

Arsenic

Selenium

Silver

Cadmium

Lead

Duplicate (9E07002-DUP1)

Chromium

Arsenic

Selenium

Silver

Cadmium

Antimony

Lead

Post Spike (9E07002-PS1)

Chromium

Arsenic

Selenium

Silver

Cadmium

Antimony

Lead
Prepared \& Analyzed: 05/07/09

$\begin{array}{lll}<3.44 \mathrm{E}-3 & 3.44 \mathrm{E}-3 & \text { ug } / \mathrm{g} \text { wet } \\ <2.84 \mathrm{E}-3 & 2.84 \mathrm{E}-3 & " \\ <7.92 \mathrm{E}-3 & 7.92 \mathrm{E}-3 & " \\ <3.13 \mathrm{E}-3 & 3.13 \mathrm{E}-3 & " \\ <5.73 \mathrm{E}-4 & 5.73 \mathrm{E}-4 & " \\ <6.58 \mathrm{E}-4 & 6.58 \mathrm{E}-4 & " \\ <1.16 \mathrm{E}-3 & 1.16 \mathrm{E}-3\end{array}$

Prepared \& Analyzed: 05/07/09

$\begin{array}{lccccc}5.13 \mathrm{E} 0 & 3.44 \mathrm{E}-1 & \text { ug/g wet } & 4.99 \mathrm{E} 0 & 103 & 80-120 \\ 5.16 \mathrm{E} 0 & 2.84 \mathrm{E}-1 & " & 4.99 \mathrm{E} 0 & 104 & 80-120 \\ 5.00 \mathrm{E} 0 & 7.92 \mathrm{E}-1 & " & 4.99 \mathrm{E} 0 & 100 & 80-120 \\ 4.94 \mathrm{E} 0 & 3.13 \mathrm{E}-1 & " & 4.99 \mathrm{E} 0 & 99.0 & 80-120 \\ 5.29 \mathrm{E} 0 & 5.73 \mathrm{E}-2 & " & 4.99 \mathrm{E} 0 & 106 & 80-120 \\ 4.72 \mathrm{E} 0 & 1.16 \mathrm{E}-1 & " & 4.99 \mathrm{E} 0 & 94.7 & 80-120\end{array}$

Source: 0902002-03 Prepared \& Analyzed: 05/07/09

\begin{tabular}{|c|c|c|c|c|}
\hline$<3.82 \mathrm{E}-3$ & $3.82 \mathrm{E}-3$ & ug/g dry & ND & \\
\hline $3.42 \mathrm{E}-3$ & $3.14 \mathrm{E}-3$ & $"$ & ND & \\
\hline$<8.77 \mathrm{E}-3$ & $8.77 \mathrm{E}-3$ & $"$ & ND & \\
\hline$<3.46 \mathrm{E}-3$ & $3.46 \mathrm{E}-3$ & $"$ & ND & \\
\hline$<6.35 \mathrm{E}-4$ & $6.35 \mathrm{E}-4$ & $"$ & ND & \\
\hline $2.32 \mathrm{E}-3$ & 7.30E-4 & $"$ & $1.47 \mathrm{E}-3$ & 44.5 \\
\hline$<1.29 \mathrm{E}-3$ & $1.29 \mathrm{E}-3$ & $"$ & ND & \\
\hline
\end{tabular}

Source: 0902002-03 Prepared \& Analyzed: 05/07/09

$\begin{array}{lcccccc}4.92 \mathrm{E} 0 & \text { N/A } & \text { ug/L } & 5.00 \mathrm{E} 0 & 1.21 \mathrm{E}-1 & 96.1 & 75-125 \\ 5.89 \mathrm{E} 0 & \text { N/A } & " & 5.00 \mathrm{E} 0 & 5.61 \mathrm{E}-1 & 107 & 75-125 \\ 5.51 \mathrm{E} 0 & \text { N/A } & " & 5.00 \mathrm{E} 0 & 2.01 \mathrm{E}-1 & 106 & 75-125 \\ 5.00 \mathrm{E} 0 & \text { N/A } & " & 5.00 \mathrm{E} 0 & \text { ND } & 100 & 75-125 \\ 5.24 \mathrm{E} 0 & \text { N/A } & " & 5.00 \mathrm{E} 0 & \text { ND } & 107 & 75-125 \\ 5.61 \mathrm{E} 0 & \text { N/A } & " & 5.00 \mathrm{E} 0 & 2.66 \mathrm{E}-1 & 107 & 75-125 \\ 5.04 \mathrm{E} 0 & \text { N/A } & " & 5.00 \mathrm{E} 0 & 6.95 \mathrm{E}-2 & 99.4 & 75-125\end{array}$


RCRA Metals By PNNL-AGG-415/Water Extract - Quality Control

Environmental Science Laboratory

\begin{tabular}{|lrrrrrrrrrrrr}
\hline & & Reporting & & Spike & Source & & & $\%$ REC & & RPD \\
Analyte & Result & Limit & Units & Level & Result & $\%$ REC & Limits & RPD & Limit & Notes \\
\hline
\end{tabular}

Batch 9E07003 - 1:1 Water Extract (ICP/ICPMS)

Blank (9E07003-BLK1)

Chromium

Arsenic

Selenium

Silver

Cadmium

Antimony

Lead

LCS (9E07003-BS1)

Chromium

Arsenic

Selenium

Silver

Cadmium

Lead

Duplicate (9E07003-DUP1)

Chromium

Arsenic

Selenium

Silver

Cadmium

Antimony

Lead

Post Spike (9E07003-PS1)

Chromium

Arsenic

Selenium

Silver

Cadmium

Antimony

Lead
Prepared \& Analyzed: 05/07/09

$\begin{array}{lll}<3.44 \mathrm{E}-3 & 3.44 \mathrm{E}-3 & \text { ug } / \mathrm{g} \text { wet } \\ <2.84 \mathrm{E}-3 & 2.84 \mathrm{E}-3 & " \\ <7.92 \mathrm{E}-3 & 7.92 \mathrm{E}-3 & " \\ <3.13 \mathrm{E}-3 & 3.13 \mathrm{E}-3 & " \\ <5.73 \mathrm{E}-4 & 5.73 \mathrm{E}-4 & " \\ <6.58 \mathrm{E}-4 & 6.58 \mathrm{E}-4 & " \\ <1.16 \mathrm{E}-3 & 1.16 \mathrm{E}-3\end{array}$

Prepared \& Analyzed: 05/07/09

$\begin{array}{lccccc}5.18 \mathrm{E} 0 & 3.44 \mathrm{E}-1 & \text { ug/g wet } & 4.99 \mathrm{E} 0 & 104 & 80-120 \\ 5.28 \mathrm{E} 0 & 2.84 \mathrm{E}-1 & " & 4.99 \mathrm{E} 0 & 106 & 80-120 \\ 5.08 \mathrm{E} 0 & 7.92 \mathrm{E}-1 & " & 4.99 \mathrm{E} 0 & 102 & 80-120 \\ 4.91 \mathrm{E} 0 & 3.13 \mathrm{E}-1 & " & 4.99 \mathrm{E} 0 & 98.5 & 80-120 \\ 5.29 \mathrm{E} 0 & 5.73 \mathrm{E}-2 & " & 4.99 \mathrm{E} 0 & 106 & 80-120 \\ 4.78 \mathrm{E} 0 & 1.16 \mathrm{E}-1 & " & 4.99 \mathrm{E} 0 & 95.8 & 80-120\end{array}$

Source: 0902002-08 Prepared \& Analyzed: 05/07/09

$\begin{array}{rccccc}1.28 \mathrm{E}-1 & 3.50 \mathrm{E}-3 & \text { ug/g dry } & 1.27 \mathrm{E}-1 & 0.758 & 35 \\ 7.89 \mathrm{E}-3 & 2.88 \mathrm{E}-3 & " & 7.51 \mathrm{E}-3 & 4.91 & 35 \\ <8.04 \mathrm{E}-3 & 8.04 \mathrm{E}-3 & " & \text { ND } & & 35 \\ <3.18 \mathrm{E}-3 & 3.18 \mathrm{E}-3 & " & \mathrm{ND} & 35 \\ <5.82 \mathrm{E}-4 & 5.82 \mathrm{E}-4 & " & \mathrm{ND} & 35 \\ <6.69 \mathrm{E}-4 & 6.69 \mathrm{E}-4 & " & \mathrm{ND} & 35 \\ <1.18 \mathrm{E}-3 & 1.18 \mathrm{E}-3 & " & \mathrm{ND} & & 35\end{array}$

Source: 0902002-08 Prepared \& Analyzed: 05/07/09

$\begin{array}{llccccc}2.91 \mathrm{E} 1 & \text { N/A } & \text { ug/L } & 5.00 \mathrm{E} 0 & 2.53 \mathrm{E} 1 & 75.5 & 75-125 \\ 7.01 \mathrm{E} 0 & \text { N/A } & " & 5.00 \mathrm{E} 0 & 1.50 \mathrm{E} 0 & 110 & 75-125 \\ 5.75 \mathrm{E} 0 & \text { N/A } & " & 5.00 \mathrm{E} 0 & 2.14 \mathrm{E}-1 & 111 & 75-125 \\ 4.95 \mathrm{E} 0 & \text { N/A } & " & 5.00 \mathrm{E} 0 & \text { ND } & 99.3 & 75-125 \\ 5.17 \mathrm{E} 0 & \text { N/A } & " & 5.00 \mathrm{E} 0 & \text { ND } & 106 & 75-125 \\ 5.31 \mathrm{E} 0 & \text { N/A } & " & 5.00 \mathrm{E} 0 & 1.50 \mathrm{E}-2 & 106 & 75-125 \\ 5.06 \mathrm{E} 0 & \text { N/A } & " & 5.00 \mathrm{E} 0 & 3.85 \mathrm{E}-2 & 100 & 75-125\end{array}$


RCRA Metals By PNNL-AGG-415/Water Extract - Quality Control

Environmental Science Laboratory

\begin{tabular}{|lrrrrrrrrrrrr}
\hline & & Reporting & & Spike & Source & & & $\%$ REC & & RPD \\
Analyte & Result & Limit & Units & Level & Result & $\%$ REC & Limits & RPD & Limit & Notes \\
\hline
\end{tabular}

Batch 9E07004 - 1:1 Water Extract (ICP/ICPMS)

Blank (9E07004-BLK1)

Chromium

Arsenic

Selenium

Silver

Cadmium

Antimony

Lead

LCS (9E07004-BS1)

Chromium

Arsenic

Selenium

Silver

Cadmium

Lead

Duplicate (9E07004-DUP1)

Chromium

Arsenic

Selenium

Silver

Cadmium

Antimony

Lead

Post Spike (9E07004-PS1)

Chromium

Arsenic

Selenium

Silver

Cadmium

Antimony

Lead
Prepared \& Analyzed: 05/07/09

$\begin{array}{rlc}<3.44 \mathrm{E}-3 & 3.44 \mathrm{E}-3 & \text { ug } / \mathrm{g} \text { wet } \\ <2.84 \mathrm{E}-3 & 2.84 \mathrm{E}-3 & " \\ <7.92 \mathrm{E}-3 & 7.92 \mathrm{E}-3 & " \\ <3.13 \mathrm{E}-3 & 3.13 \mathrm{E}-3 & " \\ <5.73 \mathrm{E}-4 & 5.73 \mathrm{E}-4 & " \\ 1.40 \mathrm{E}-2 & 6.58 \mathrm{E}-4 & " \\ <1.16 \mathrm{E}-3 & 1.16 \mathrm{E}-3\end{array}$

Prepared \& Analyzed: 05/07/09

$\begin{array}{lccccc}5.08 \mathrm{E} 0 & 3.44 \mathrm{E}-1 & \text { ug/g wet } & 5.00 \mathrm{E} 0 & 102 & 80-120 \\ 5.24 \mathrm{E} 0 & 2.84 \mathrm{E}-1 & " & 5.00 \mathrm{E} 0 & 105 & 80-120 \\ 5.05 \mathrm{E} 0 & 7.92 \mathrm{E}-1 & " & 5.00 \mathrm{E} 0 & 101 & 80-120 \\ 4.97 \mathrm{E} 0 & 3.13 \mathrm{E}-1 & " & 5.00 \mathrm{E} 0 & 99.3 & 80-120 \\ 5.34 \mathrm{E} 0 & 5.73 \mathrm{E}-2 & " & 5.00 \mathrm{E} 0 & 107 & 80-120 \\ 4.82 \mathrm{E} 0 & 1.16 \mathrm{E}-1 & " & 5.00 \mathrm{E} 0 & 96.5 & 80-120\end{array}$

Source: 0902002-12 Prepared \& Analyzed: 05/07/09

$\begin{array}{llcll}<3.47 \mathrm{E}-3 & 3.47 \mathrm{E}-3 & \text { ug/g dry } & \text { ND } & 35 \\ <2.86 \mathrm{E}-3 & 2.86 \mathrm{E}-3 & " & \text { ND } & 35 \\ <7.98 \mathrm{E}-3 & 7.98 \mathrm{E}-3 & " & \text { ND } & 35 \\ <3.15 \mathrm{E}-3 & 3.15 \mathrm{E}-3 & " & \text { ND } & 35 \\ <5.78 \mathrm{E}-4 & 5.78 \mathrm{E}-4 & " & \text { ND } & 35 \\ <6.64 \mathrm{E}-4 & 6.64 \mathrm{E}-4 & " & \text { ND } & 35 \\ <1.17 \mathrm{E}-3 & 1.17 \mathrm{E}-3 & " & \text { ND } & \end{array}$

Source: 0902002-12 Prepared \& Analyzed: 05/07/09

$\begin{array}{lllllll}4.97 \mathrm{E} 0 & \text { N/A } & \text { ug/L } & 5.00 \mathrm{E} 0 & 2.66 \mathrm{E}-1 & 94 & 75-125 \\ 6.05 \mathrm{E} 0 & \text { N/A } & " & 5.00 \mathrm{E} 0 & 3.42 \mathrm{E}-1 & 114 & 75-125 \\ 5.78 \mathrm{E} 0 & \text { N/A } & " & 5.00 \mathrm{E} 0 & 1.43 \mathrm{E}-1 & 113 & 75-125 \\ 5.00 \mathrm{E} 0 & \text { N/A } & " & 5.00 \mathrm{E} 0 & \text { ND } & 100 & 75-125 \\ 5.24 \mathrm{E} 0 & \text { N/A } & " & 5.00 \mathrm{E} 0 & \text { ND } & 107 & 75-125 \\ 5.34 \mathrm{E} 0 & \text { N/A } & " & 5.00 \mathrm{E} 0 & 1.88 \mathrm{E}-2 & 106 & 75-125 \\ 5.11 \mathrm{E} 0 & \text { N/A } & " & 5.00 \mathrm{E} 0 & 5.74 \mathrm{E}-2 & 101 & 75-125\end{array}$


RCRA Metals By PNNL-AGG-415/Acid Extract - Quality Control

Environmental Science Laboratory

\begin{tabular}{|lrrrrrrrrrrr}
\hline & & Reporting & & Spike & Source & & \%REC & & RPD \\
Analyte & Result & Limit & Units & Level & Result & \%REC & Limits & RPD & Limit & Notes \\
\hline
\end{tabular}

Batch 9E07006 - ASTM D 5198 (ICP/ICPMS)

\begin{tabular}{|c|c|c|c|c|c|c|c|c|c|}
\hline \multirow{2}{*}{$\begin{array}{l}\text { Blank (9E07006-BLK1) } \\
\text { Chromium }\end{array}$} & & & & \multicolumn{2}{|c|}{ Prepared: 05/07/09 } & \multicolumn{2}{|c|}{ Analyzed: 05/08/09 } & & \\
\hline & $<1.88 \mathrm{E}-1$ & $1.88 \mathrm{E}-1$ & ug/g wet & & & & & & \\
\hline Arsenic & $<8.20 \mathrm{E}-2$ & $8.20 \mathrm{E}-2$ & $"$ & & & & & & \\
\hline Selenium & $<1.59 \mathrm{E}-1$ & $1.59 \mathrm{E}-1$ & $"$ & & & & & & \\
\hline Silver & $<5.13 \mathrm{E}-3$ & $5.13 \mathrm{E}-3$ & $"$ & & & & & & \\
\hline Cadmium & $<1.35 \mathrm{E}-2$ & $1.35 \mathrm{E}-2$ & $"$ & & & & & & \\
\hline Antimony & $<5.26 \mathrm{E}-2$ & $5.26 \mathrm{E}-2$ & $"$ & & & & & & \\
\hline Lead & $<5.13 \mathrm{E}-2$ & $5.13 \mathrm{E}-2$ & $"$ & & & & & & \\
\hline \multicolumn{4}{|l|}{ LCS (9E07006-BS1) } & \multicolumn{2}{|c|}{ Prepared: 05/07/09 } & \multicolumn{2}{|c|}{ Analyzed: 05/08/09 } & & \\
\hline Chromium & $5.79 \mathrm{E} 0$ & $9.42 \mathrm{E}-1$ & ug/g wet & $5.94 \mathrm{E} 0$ & & 97.5 & $80-120$ & & \\
\hline Arsenic & $5.74 \mathrm{E} 0$ & $4.10 \mathrm{E}-1$ & $"$ & 5.94E0 & & 96.7 & $80-120$ & & \\
\hline Selenium & 5.64E0 & $7.93 \mathrm{E}-1$ & $"$ & $5.94 \mathrm{E} 0$ & & 95.1 & $80-120$ & & \\
\hline Silver & $5.56 \mathrm{E} 0$ & $2.56 \mathrm{E}-2$ & $"$ & $5.94 \mathrm{E} 0$ & & 93.7 & $80-120$ & & \\
\hline Cadmium & $5.72 \mathrm{E} 0$ & $6.77 \mathrm{E}-2$ & $"$ & $5.94 \mathrm{E} 0$ & & 96.4 & $80-120$ & & \\
\hline Antimony & $6.81 \mathrm{E} 0$ & $2.63 \mathrm{E}-1$ & $"$ & $5.94 \mathrm{E} 0$ & & 115 & $80-120$ & & \\
\hline Lead & $5.48 \mathrm{E} 0$ & $2.57 \mathrm{E}-1$ & $"$ & $5.94 \mathrm{E} 0$ & & 92.2 & $80-120$ & & \\
\hline Duplicate (9E07006-DUP1) & \multicolumn{3}{|c|}{ Source: 0902002-08 } & \multicolumn{2}{|c|}{ Prepared: 05/07/09 } & \multicolumn{2}{|c|}{ Analyzed: 05/11/09 } & & \\
\hline Chromium & $1.77 \mathrm{E} 1$ & $7.30 \mathrm{E}-1$ & ug/g dry & & $1.78 \mathrm{E} 1$ & & & 0.369 & 35 \\
\hline Arsenic & $5.54 \mathrm{E} 0$ & $3.18 \mathrm{E}-1$ & $"$ & & $5.50 \mathrm{E} 0$ & & & 0.647 & 35 \\
\hline Selenium & $<6.14 \mathrm{E}-1$ & $6.14 \mathrm{E}-1$ & $"$ & & ND & & & & 35 \\
\hline Silver & $6.31 \mathrm{E}-2$ & $1.99 \mathrm{E}-2$ & $"$ & & $6.32 \mathrm{E}-2$ & & & 0.166 & 35 \\
\hline Cadmium & $6.39 \mathrm{E}-2$ & $5.25 \mathrm{E}-2$ & $"$ & & $6.69 \mathrm{E}-2$ & & & 4.58 & 35 \\
\hline Antimony & $<2.04 \mathrm{E}-1$ & $2.04 \mathrm{E}-1$ & $"$ & & ND & & & & 35 \\
\hline Lead & $6.04 \mathrm{E} 0$ & $1.99 \mathrm{E}-1$ & $"$ & & $6.02 \mathrm{E} 0$ & & & 0.330 & 35 \\
\hline Post Spike (9E07006-PS1) & \multicolumn{3}{|c|}{ Source: 0902002-08 } & \multicolumn{2}{|c|}{ Prepared: 05/07/09 } & \multicolumn{2}{|c|}{ Analyzed: 05/11/09 } & & \\
\hline Chromium & 4.94E1 & N/A & $\mathrm{ug} / \mathrm{L}$ & $5.00 \mathrm{E} 0$ & $4.55 \mathrm{E} 1$ & 79.3 & $75-125$ & & \\
\hline Arsenic & $1.86 \mathrm{E} 1$ & N/A & $"$ & $5.00 \mathrm{E} 0$ & $1.40 \mathrm{E} 1$ & 90.8 & $75-125$ & & \\
\hline Selenium & $3.50 \mathrm{E} 0$ & $\mathrm{~N} / \mathrm{A}$ & $"$ & $5.00 \mathrm{E} 0$ & ND & 79.7 & $75-125$ & & \\
\hline Silver & $4.98 \mathrm{E} 0$ & N/A & $"$ & $5.00 \mathrm{E} 0$ & $1.61 \mathrm{E}-1$ & 96.4 & $75-125$ & & \\
\hline Cadmium & $4.92 \mathrm{E} 0$ & N/A & $"$ & $5.00 \mathrm{E} 0$ & $1.71 \mathrm{E}-1$ & 94.9 & $75-125$ & & \\
\hline Antimony & 5.17E0 & N/A & $"$ & $5.00 \mathrm{E} 0$ & $1.87 \mathrm{E}-1$ & 99.6 & $75-125$ & & \\
\hline Lead & $1.86 \mathrm{E} 1$ & N/A & $"$ & $5.00 \mathrm{E} 0$ & $1.54 \mathrm{E} 1$ & 65.4 & $75-125$ & & \\
\hline
\end{tabular}




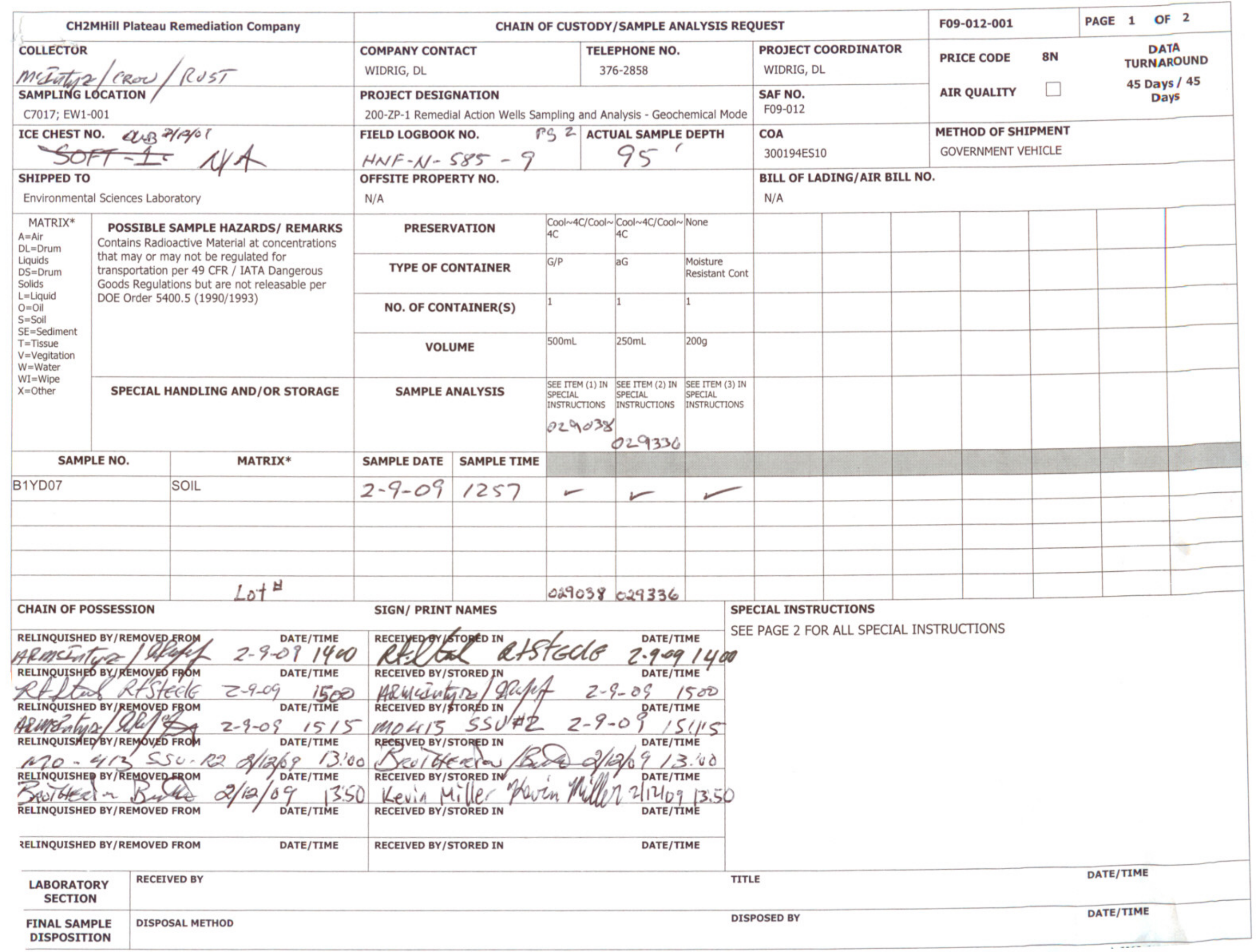




\begin{tabular}{|c|c|c|c|c|c|c|c|}
\hline CH2MHill Plateau Remediation Company & \multicolumn{3}{|c|}{ CHAIN OF CUSTODY/SAMPLE ANALYSIS REQUEST } & F09-012-001 & & PAGE & 2 OF 2 \\
\hline $\begin{array}{l}\text { COLLECTOR } \\
\text { MEIutura Cos }\end{array}$ & $\begin{array}{l}\text { COMPANY CONTACT } \\
\text { WIDRIG, DL }\end{array}$ & $\begin{array}{l}\text { TELEPHONE NO. } \\
376-2858\end{array}$ & $\begin{array}{l}\text { PROJECT COORDINATOR } \\
\text { WIDRIG, DL }\end{array}$ & \multirow{2}{*}{$\begin{array}{l}\text { PRICE CODE } \\
\text { AIR QUALITY }\end{array}$} & $8 \mathrm{~N}$ & \multirow{2}{*}{\multicolumn{2}{|c|}{$\begin{array}{c}\text { DATA } \\
\text { TURNAROUND } \\
45 \text { Days / } 45 \\
\text { Days }\end{array}$}} \\
\hline $\begin{array}{l}\text { SAMPLING LOCATION } \\
\text { C7017; EW1-001 }\end{array}$ & \multicolumn{2}{|c|}{$\begin{array}{l}\text { PROJECT DESIGNATION } \\
\text { 200-ZP-1 Remedial Action Wells Sampling and Analysis - Geochemical Mode }\end{array}$} & $\begin{array}{l}\text { SAF NO. } \\
\text { F09-012 }\end{array}$ & & $\square$ & & \\
\hline $\begin{array}{l}\text { ICE CHEST NO. DW B } \\
\text { SOFI }\end{array}$ & $\begin{array}{l}\text { FIELD LOGBOOK NO. } P_{g} 2 \\
\text { HNF-N/-585-9 }\end{array}$ & $\begin{array}{l}\text { ACTUAL SAMPLE DEPTH } \\
95\end{array}$ & $\begin{array}{l}\text { COA } \\
300194 E S 10\end{array}$ & \multicolumn{4}{|c|}{$\begin{array}{l}\text { METHOD OF SHIPMENT } \\
\text { GOVERNMENT VEHICLE }\end{array}$} \\
\hline $\begin{array}{l}\text { SHIPPED TO } \\
\text { Environmental Sciences Laboratory }\end{array}$ & \multicolumn{2}{|l|}{$\begin{array}{l}\text { OFFSITE PROPERTY NO. } \\
\text { N/A }\end{array}$} & \multicolumn{3}{|c|}{$\begin{array}{l}\text { BILL OF LADING/AIR BILL NO. } \\
\text { N/A }\end{array}$} & & \\
\hline
\end{tabular}

\section{SPECIAL INSTRUCTIONS}

** The 200 Area S\&GRP Characterization and Monitoring Sampling and Analysis GKI applies to this SAF.

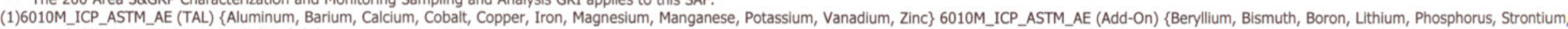

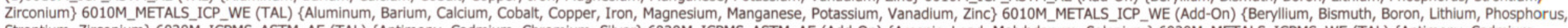

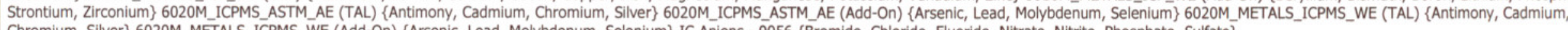
Chromium, Silver\} 6020M_METALS_ICPMS_WE (Add-On) \{Arsenic, Lead, Molybdenum, Selenium\} IC Anions - 9056 \{Bromide, Chloride, Fluoride, Nitrate, Nitrite, Phosphate, Sulfate\}

(2)TOC - ASTME1915A \{Total organic carbon\} GAMMA_GS \{Cesium-137, Cobalt-60, Europium-152, Europium-154, Europium-155

(3)Moisture Content - D2216 \{Percent moisture (wet sample)\} 


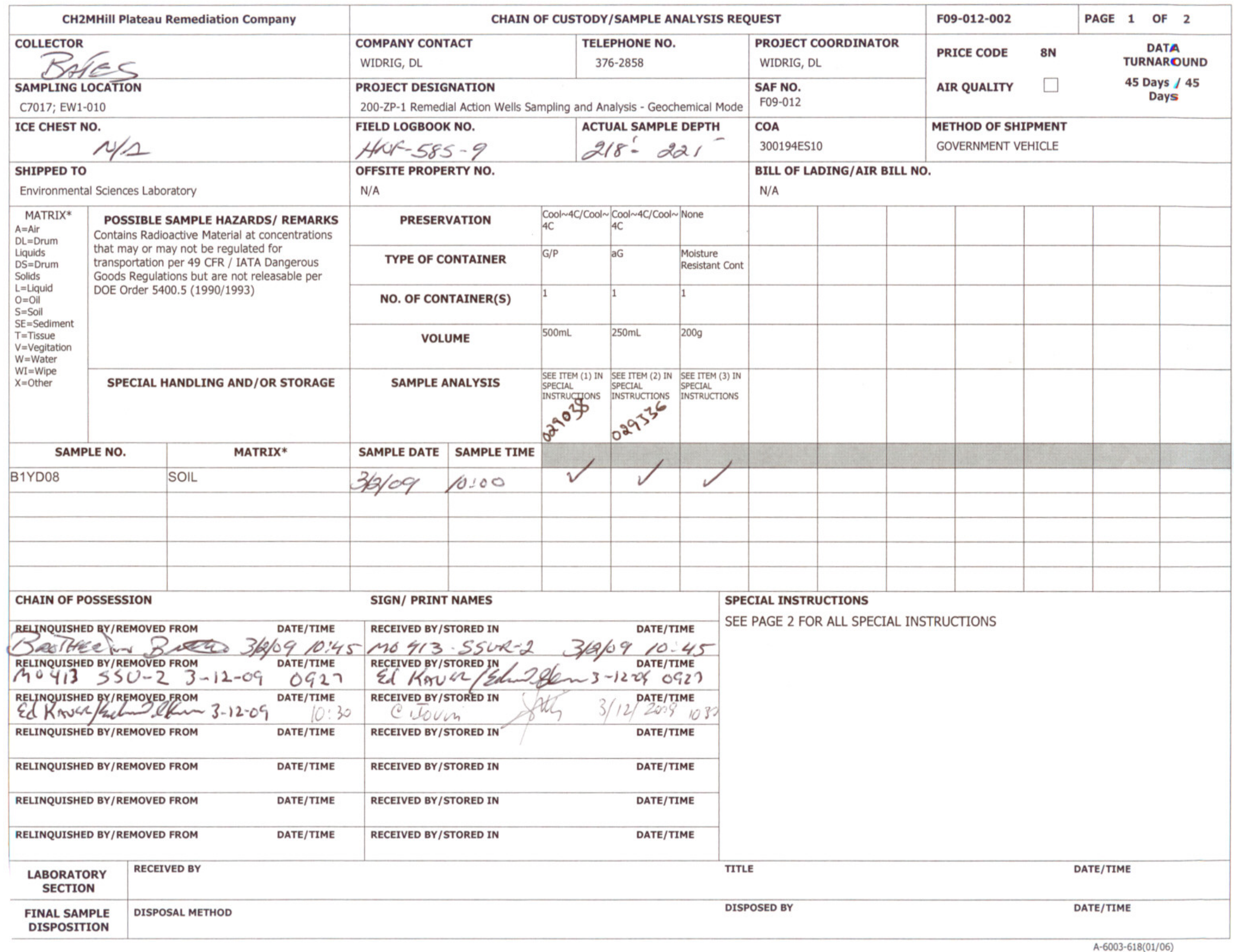




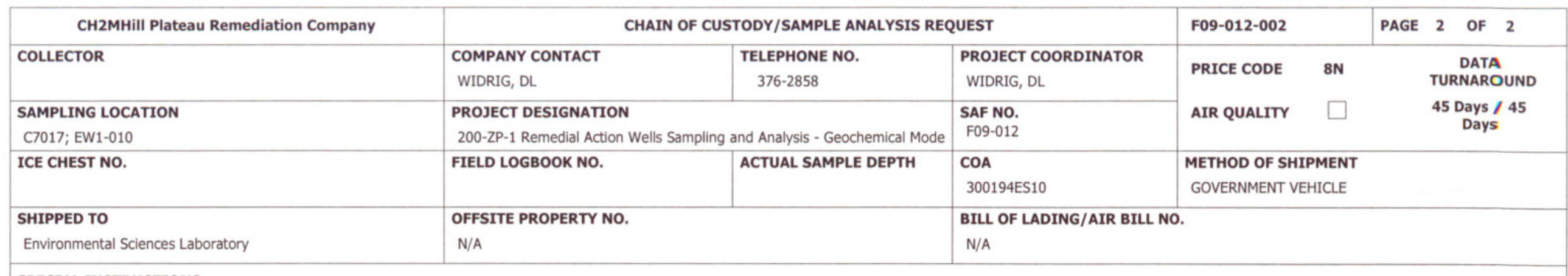

\section{SPECIAL INSTRUCTIONS}

** The 200 Area S\&GRP Characterization and Monitoring Sampling and Analysis GKI applies to this SAF.

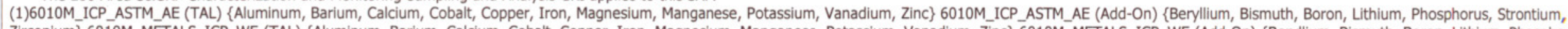

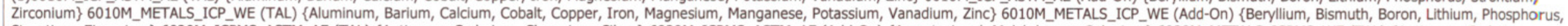

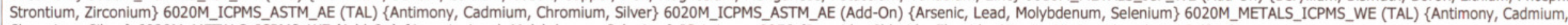
Chromium, Silver\} 6020M_METALS_ICPMS_WE (Add-On) \{Arsenic, Lead, Molybdenum, Selenium\} IC Anions - 9056 \{Bromide, Chloride, Fluoride, Nitrate, Nitrite, Phosphate, Sulfate\}

(2)TOC - ASTME1915A \{Total organic carbon\} GAMMA_GS \{Cesium-137, Cobalt-60, Europium-152, Europium-154, Europium-155\}

(3) Moisture Content - D2216 \{Percent moisture (wet sample)\} 


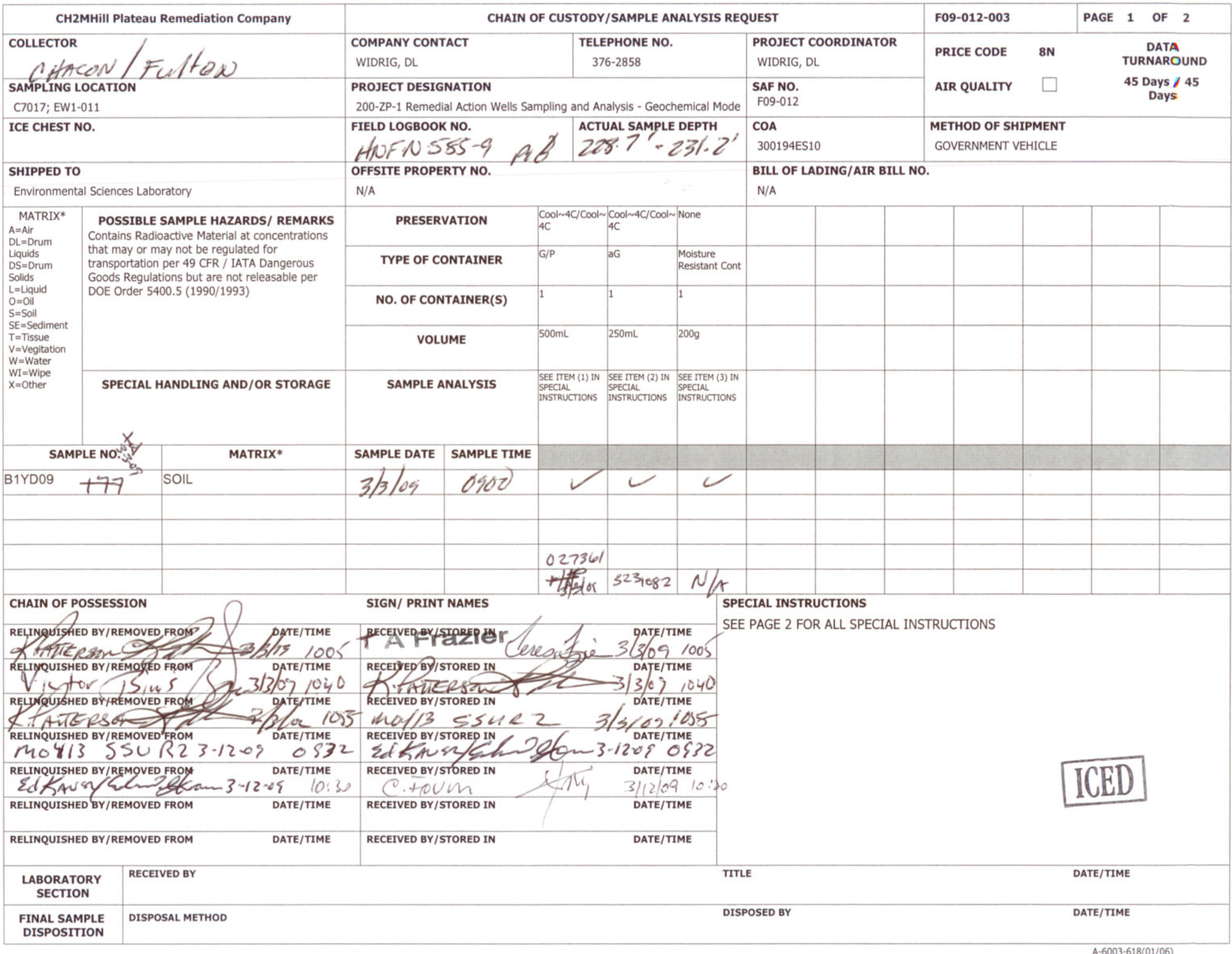




\begin{tabular}{|c|c|c|c|c|c|c|c|}
\hline CH2MHill Plateau Remediation Company & \multicolumn{3}{|c|}{ CHAIN OF CUSTODY/SAMPLE ANALYSIS REQUEST } & \multicolumn{2}{|l|}{ F09-012-003 } & PAGE & 2 OF 2 \\
\hline COLLECTOR & $\begin{array}{l}\text { COMPANY CONTACT } \\
\text { WIDRIG, DL }\end{array}$ & $\begin{array}{l}\text { TELEPHONE NO. } \\
376-2858\end{array}$ & $\begin{array}{l}\text { PROJECT COORDINATOR } \\
\text { WIDRIG, DL }\end{array}$ & \multirow{2}{*}{$\begin{array}{l}\text { PRICE CODE } \\
\text { AIR QUALITY }\end{array}$} & \multirow{2}{*}{$\begin{array}{l}\mathbf{8 N} \\
\square\end{array}$} & \multirow{2}{*}{\multicolumn{2}{|c|}{$\begin{array}{c}\text { DATA } \\
\text { TURNAROUND } \\
45 \text { Days / } 45 \\
\text { Days }\end{array}$}} \\
\hline $\begin{array}{l}\text { SAMPLING LOCATION } \\
\text { C7017; EW1-011 }\end{array}$ & \multicolumn{2}{|c|}{$\begin{array}{l}\text { PROJECT DESIGNATION } \\
\text { 200-ZP-1 Remedial Action Wells Sampling and Analysis - Geochemical Mode }\end{array}$} & $\begin{array}{r}\text { SAF NO. } \\
\text { F09-012 }\end{array}$ & & & & \\
\hline ICE CHEST NO. & FIELD LOGBOOK NO. & ACTUAL SAMPLE DEPTH & $\begin{array}{l}\text { COA } \\
\text { 300194ES10 }\end{array}$ & \multicolumn{4}{|c|}{$\begin{array}{l}\text { METHOD OF SHIPMENT } \\
\text { GOVERNMENT VEHICLE }\end{array}$} \\
\hline $\begin{array}{l}\text { SHIPPED TO } \\
\text { Environmental Sciences Laboratory }\end{array}$ & \multicolumn{2}{|l|}{$\begin{array}{l}\text { OFFSITE PROPERTY NO. } \\
\text { N/A }\end{array}$} & \multicolumn{3}{|c|}{$\begin{array}{l}\text { BILL OF LADING/AIR BILL NO. } \\
\text { N/A }\end{array}$} & & \\
\hline
\end{tabular}

\section{SPECIAL INSTRUCTIONS}

** The 200 Area S\&GRP Characterization and Monitoring Sampling and Analysis GKI applies to this SAF.

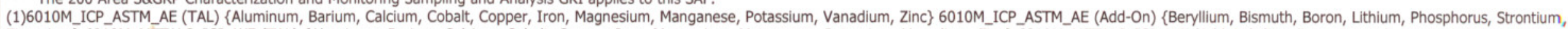

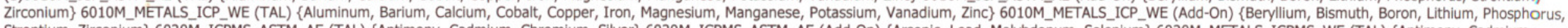

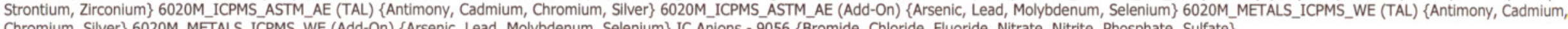
Chromium, Silver\} 6020M_METALS_ICPMS_WE (Add-On) \{Arsenic, Lead, Molybdenum, Selenium\} IC Anions - 9056 \{Bromide, Chloride, Fluoride, Nitrate, Nitrite, Phosphate, Sulfate

(2)TOC - ASTME1915A \{Total organic carbon\} GAMMA_GS \{Cesium-137, Cobalt-60, Europium-152, Europium-154, Europium-155\}

(3)Moisture Content - D2216 \{Percent moisture (wet sample)\} 


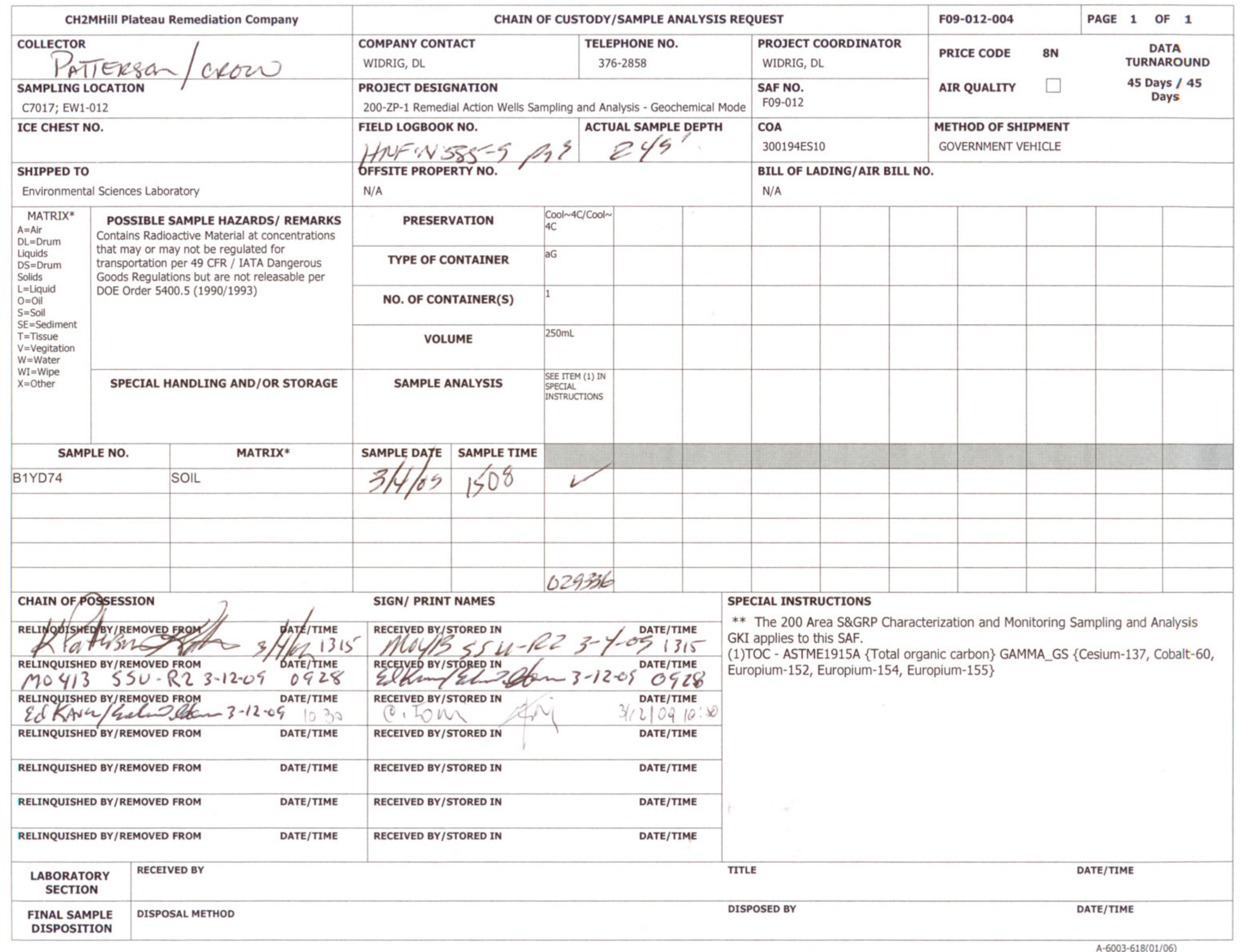




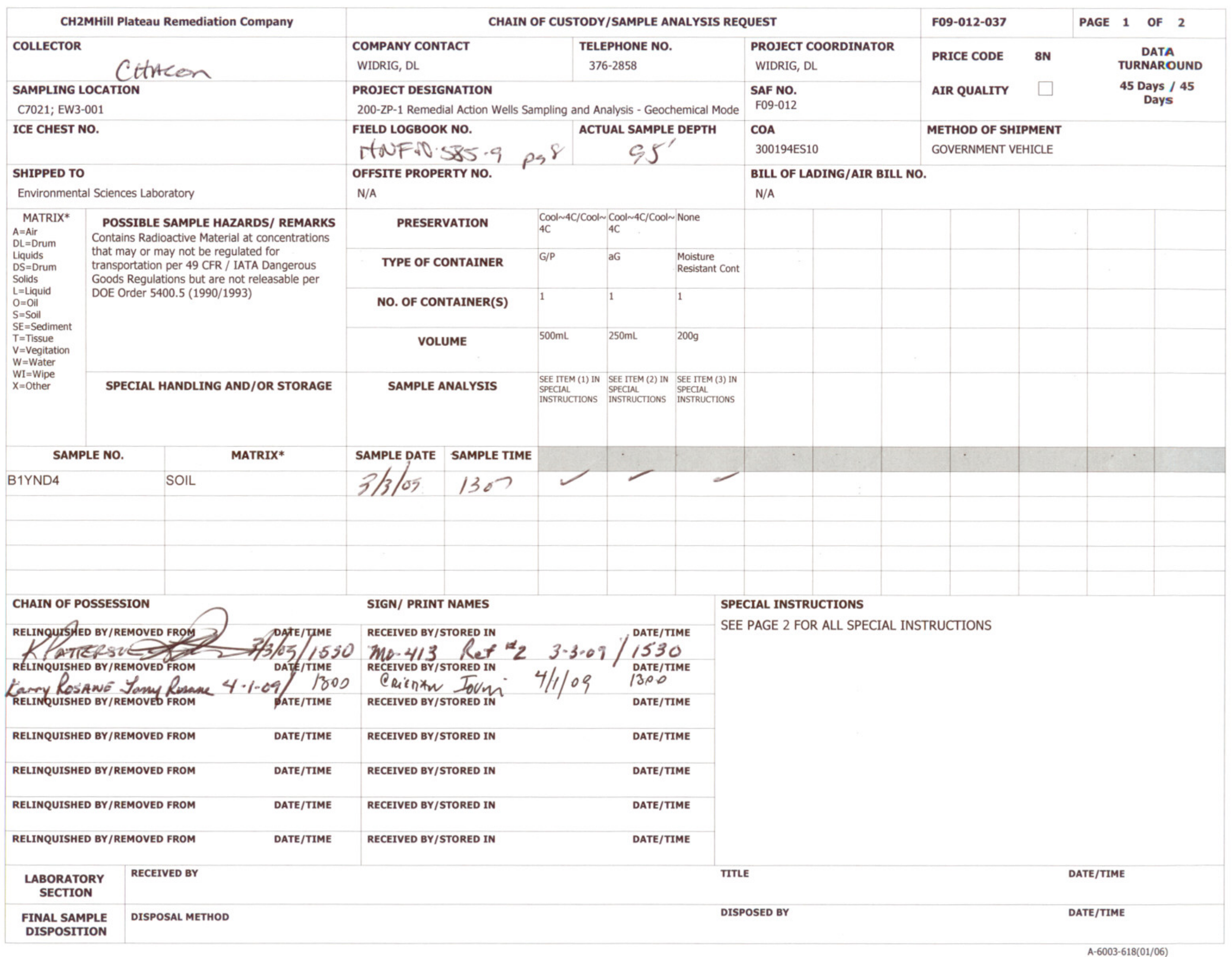




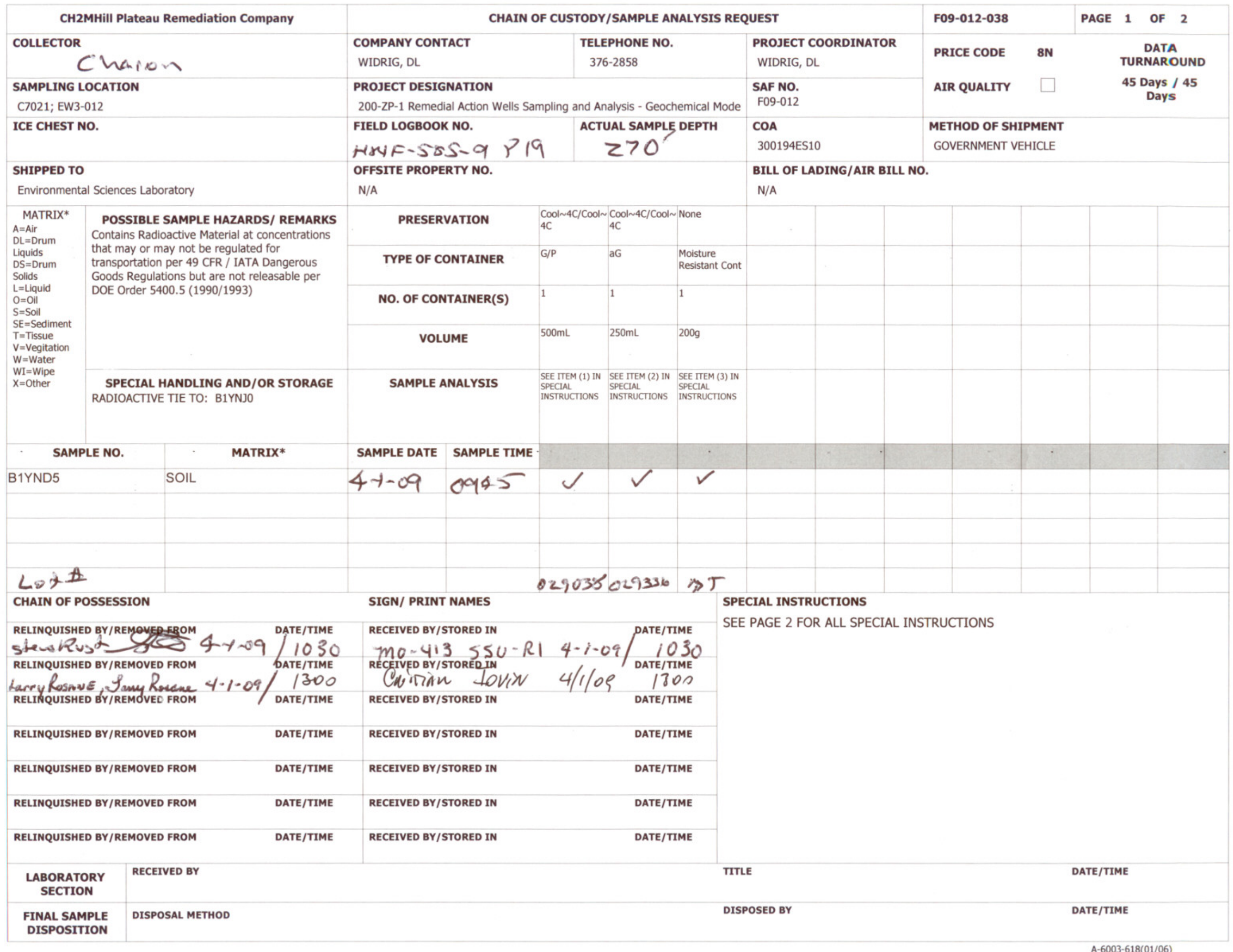




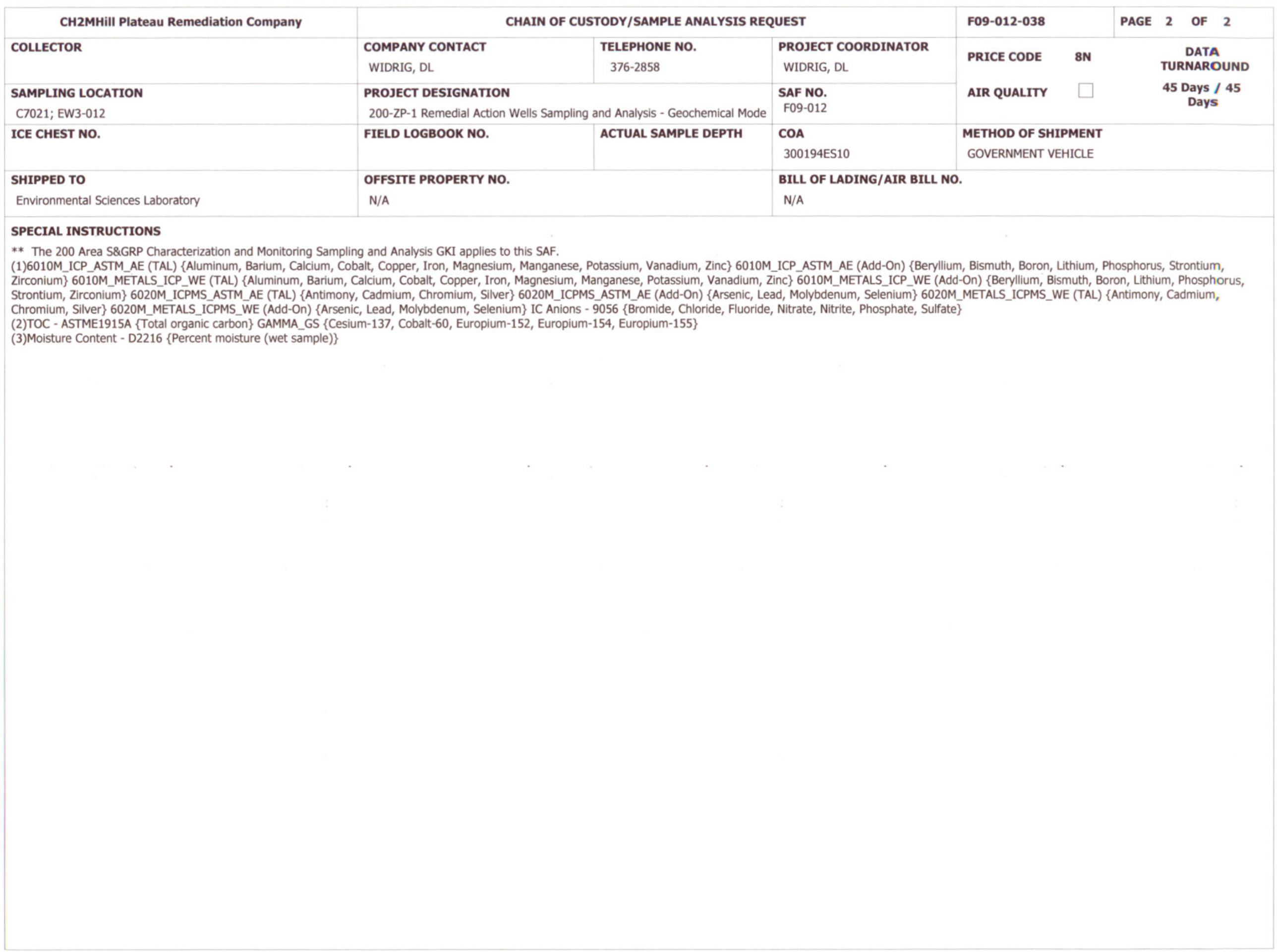


CHAIN OF CUSTODY/SAMPLE ANALYSIS REQUEST

SAMPLING LOCATION

C7021; EW3-010-G.S

ICE CHEST NO.

\section{SHIPPED TO}

Environmental Sciences Laboratory

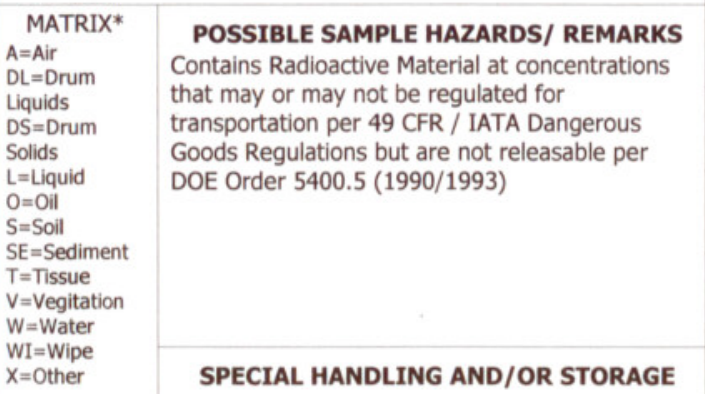

$\begin{array}{ll}\mathrm{X}=\mathrm{O} \text { ther } & \text { SPECIAL HANDLING AND/OR STORAGE }\end{array}$

\begin{tabular}{|l|l|}
\hline SAMPLE NO. & \multicolumn{1}{|c|}{ MATRIX* } \\
\hline B1YND7 & SOIL \\
\hline
\end{tabular}

\section{CHAIN OF POSSESSION}

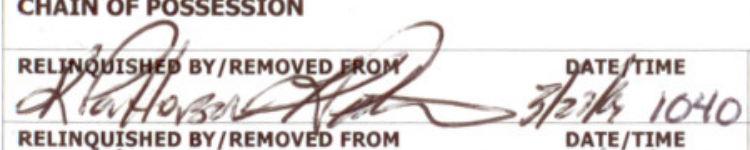
RELINQUISHED BY/REMOVED FROM DATE/TIME

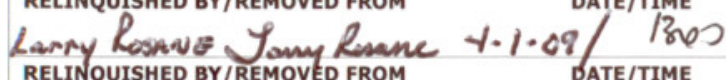
RELINQUISHED BY/REMOVED FROM

RELINQUISHED BY/REMOVED FROM DATE/TIME

RELINQUISHED BY/REMOVED FROM DATE/TIME

RELINQUISHED BY/REMOVED FROM DATE/TIME

RELINQUISHED BY/REMOVED FROM DATE/TIME

\section{COMPANY CONTACT}

WIDRIG, DL

PROJECT DESIGNATION

200-ZP-1 Remedial Action Wells Sampling and Analysis - Geochemical Mode

FIELD LOGBOOK NO.

ACTUAL SAMPLE DEPTH

\begin{tabular}{|c|}
\hline \\
\hline \\
\hline \\
\hline \\
\hline Iot \\
\hline
\end{tabular}

OPRITE PROPERTY NO.

N/A

\begin{tabular}{|c|c|c|c|}
\hline PRESERVATION & $\begin{array}{l}\text { Cool 4C/Cool } \\
4 \mathrm{C}\end{array}$ & $\begin{array}{l}\text { Cool 4C/Cool } \\
4 \mathrm{C}\end{array}$ & None \\
\hline TYPE OF CONTAINER & G/P & aG & $\begin{array}{l}\text { Moisture } \\
\text { Resistant Cont }\end{array}$ \\
\hline NO. OF CONTAINER(S) & 1 & 1 & 1 \\
\hline VOLUME & $500 \mathrm{~mL}$ & $250 \mathrm{~mL}$ & 2009 \\
\hline SAMPLE ANALYSIS & $\begin{array}{l}\text { SEE MTEM (1) IN } \\
\text { SPECIAL } \\
\text { INSTRUCTIONS }\end{array}$ & $\begin{array}{l}\text { SEE ITEM (2) IN } \\
\text { SPECIAL } \\
\text { INSTRUCTIONS }\end{array}$ & $\begin{array}{l}\text { SEE ITEM (3) IN } \\
\text { SPECIAL } \\
\text { INSTRUCTIONS }\end{array}$ \\
\hline
\end{tabular}

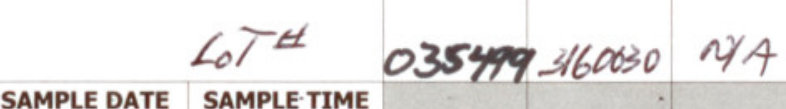

$3 / 2$ the 0932

\section{SIGN/ PRINT NAMES}

RECEIVED BY/STORED IN MO 413 SSU-R2

RECEIVED BY/STOREDIN

Cońmin to n

RECEIVED BY/STORED IN

RECEIVED BY/STORED IN

RECEIVED BY/STORED IN

RECEIVED BY/STORED IN
RECEIVED BY/STORED IN
PROJECT COORDINATOR

WIDRIG, DL

SAF NO.

F09-012

COA

300194 ES 10

BILL OF LADING/AIR BILL NO.

\begin{tabular}{|l|}
\hline F09-012-052 \\
\hline PRICE CODE \\
AIR QUALITY \\
\hline METHOD OF SHIPMENT \\
GOVERNMENT VEHICLE
\end{tabular}

PAGE 1 OF 2

TURNAROUND

45 Days / 45

Days

GOVERNMENT VEHICLE

\begin{tabular}{|c|c|c|c|}
\hline $\begin{array}{l}\text { LABORATORY } \\
\text { SECTION }\end{array}$ & RECEIVED BY & TITLE & DATE/TIME \\
\hline $\begin{array}{l}\text { FINAL SAMPLE } \\
\text { DISPOSITION }\end{array}$ & DISPOSAL METHOD & DISPOSED BY & DATE/TIME \\
\hline
\end{tabular}

SPECIAL INSTRUCTIONS

SEE PAGE 2 FOR ALL SPECIAL INSTRUCTIONS 


\begin{tabular}{|c|c|c|c|c|c|c|c|}
\hline CH2MHill Plateau Remediation Company & \multicolumn{3}{|c|}{ CHAIN OF CUSTODY/SAMPLE ANALYSIS REQUEST } & \multicolumn{2}{|l|}{ F09-012-052 } & PAGE & 2 OF 2 \\
\hline 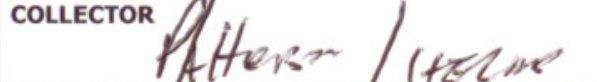 & $\begin{array}{l}\text { COMPANY CONTACT } \\
\text { WIDRIG, DL }\end{array}$ & $\begin{array}{l}\text { TELEPHONE NO. } \\
376-2858\end{array}$ & $\begin{array}{l}\text { PROJECT COORDINATOR } \\
\text { WIDRIG, DL }\end{array}$ & \multirow{2}{*}{$\begin{array}{l}\text { PRICE CODE } \\
\text { AIR QUALITY }\end{array}$} & \multicolumn{2}{|l|}{$\mathbf{8 N}$} & $\begin{array}{l}\text { DATA } \\
\text { TURNAROUND }\end{array}$ \\
\hline $\begin{array}{l}\text { SAMPLING LØCATION } \\
\text { C7021; EW3-010-G.S }\end{array}$ & \multicolumn{2}{|c|}{$\begin{array}{l}\text { PROJECT DESIGNATION } \\
\text { 200-ZP-1 Remedial Action Wells Sampling and Analysis - Geochemical Mode }\end{array}$} & $\begin{array}{l}\text { SAF NO. } \\
\text { F09-012 }\end{array}$ & & $\square$ & & $\begin{array}{l}45 \text { Days / } 45 \\
\text { Days }\end{array}$ \\
\hline ICE CHEST NO. & FIELD LOGBOOK NO. & ACTUAL SAMPLE DEPTH & $\begin{array}{l}\text { COA } \\
\text { 300194ES10 }\end{array}$ & \multicolumn{4}{|c|}{$\begin{array}{l}\text { METHOD OF SHIPMENT } \\
\text { GOVERNMENT VEHICLE }\end{array}$} \\
\hline $\begin{array}{l}\text { SHIPPED TO } \\
\text { Environmental Sciences Laboratory }\end{array}$ & \multicolumn{2}{|c|}{$\begin{array}{l}\text { OFFSITE PROPERTY NO. } \\
\text { N/A }\end{array}$} & \multicolumn{3}{|c|}{$\begin{array}{l}\text { BILL OF LADING/AIR BILL NO. } \\
\text { N/A }\end{array}$} & & \\
\hline
\end{tabular}

\section{SPECIAL INSTRUCTIONS}

** The 200 Area S\&GRP Characterization and Monitoring Sampling and Analysis GKI applies to this SAF.

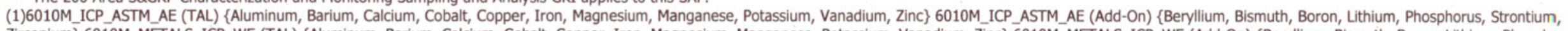

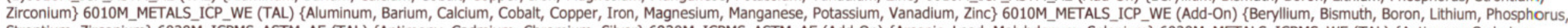

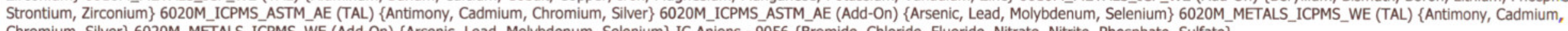
Chromium, Silver\} 6020M_METALS_ICPMS_WE (Add-On) \{Arsenic, Lead, Molybdenum, Selenium\} IC Anions - 9056 \{Bromide, Chloride, Fluoride, Nitrate, Nitrite, Phosphate, Sulfate\}

(2)TOC - ASTME1915A \{Total organic carbon\} GAMMA_GS \{Cesium-137, Cobalt-60, Europium-152, Europium-154, Europium-155\}

(3)Moisture Content - D2216 \{Percent moisture (wet sample)\} 


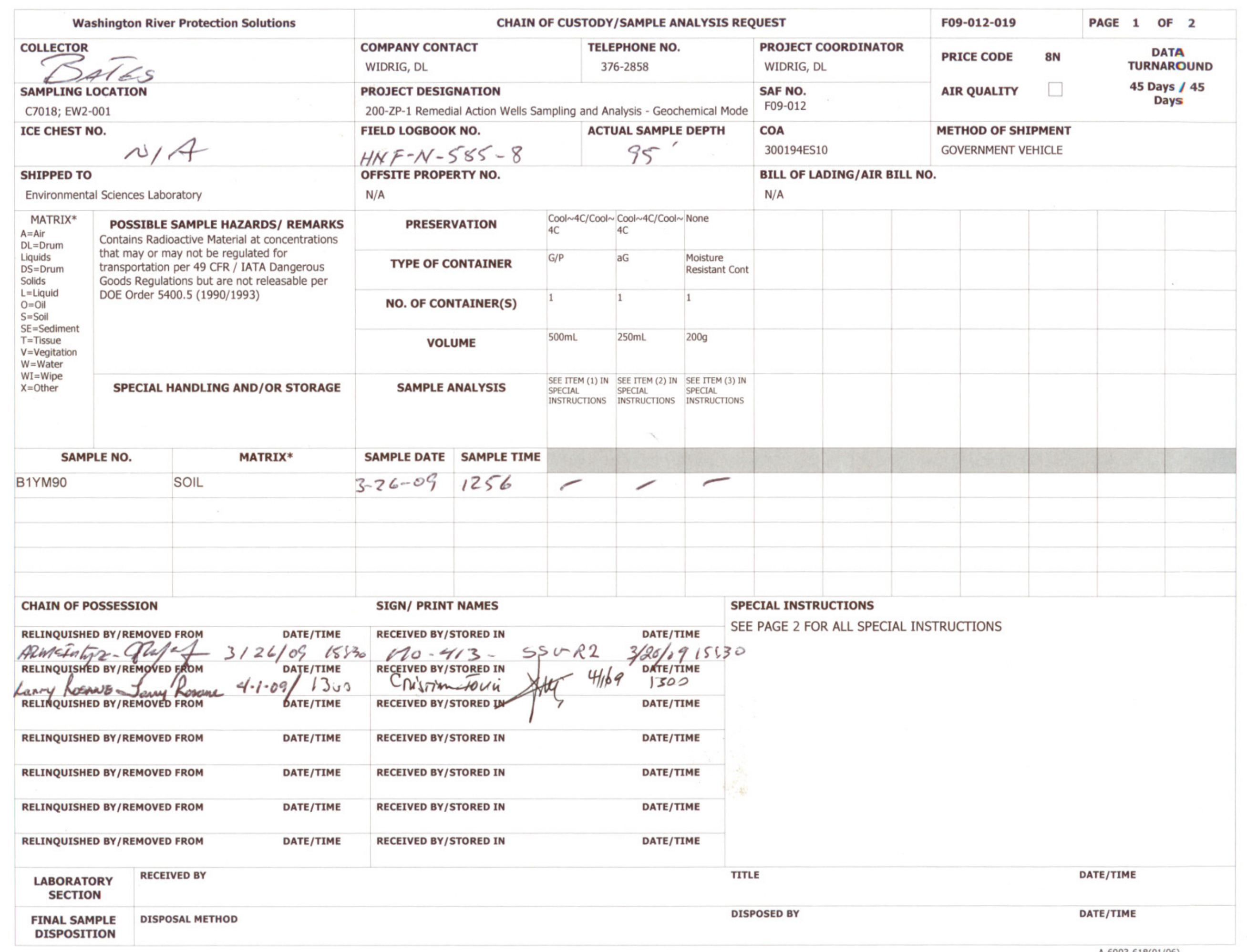




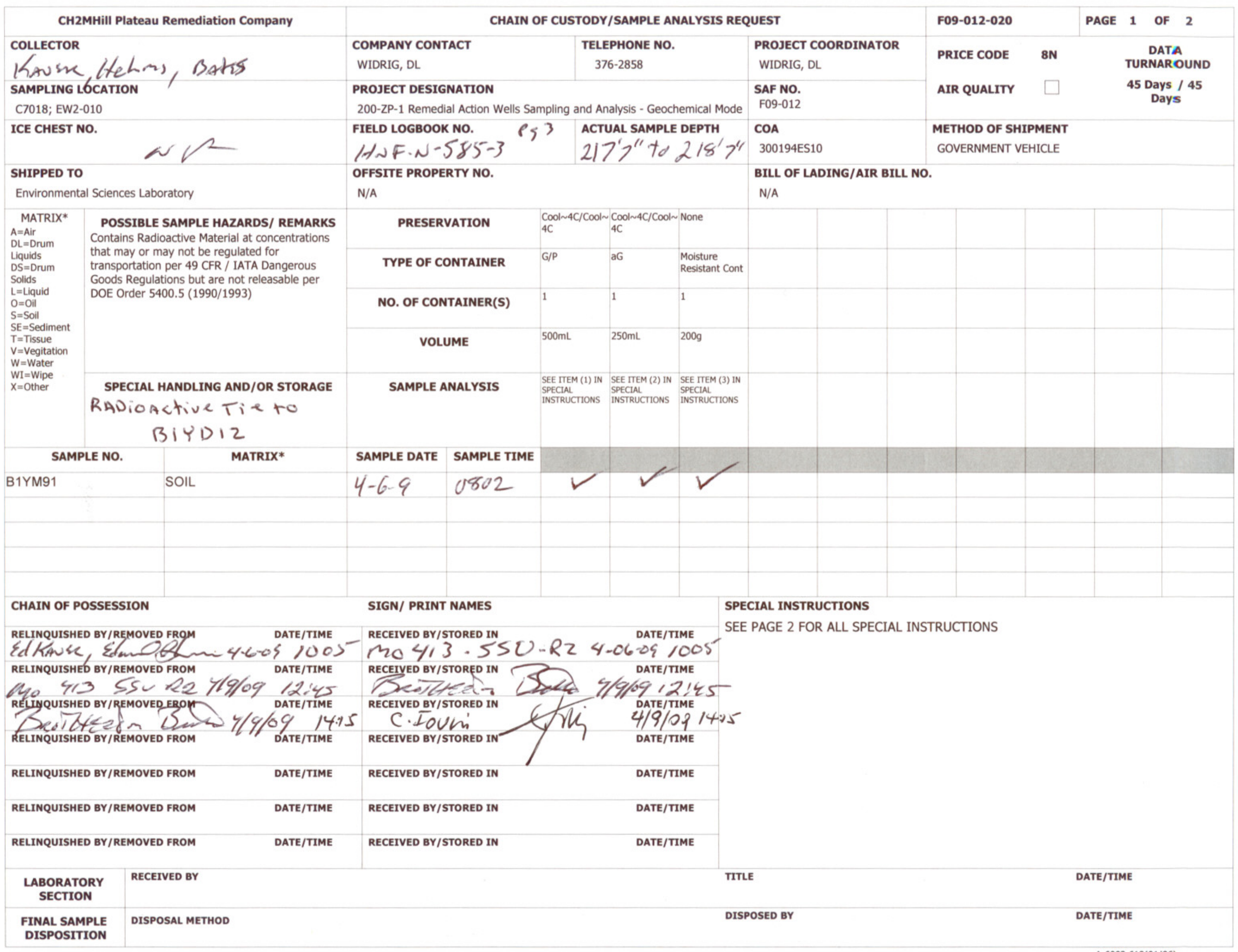




\begin{tabular}{|c|c|c|c|c|c|c|c|}
\hline CH2MHill Plateau Remediation Company & \multicolumn{3}{|c|}{ CHAIN OF CUSTODY/SAMPLE ANALYSIS REQUEST } & \multicolumn{2}{|l|}{ F09-012-020 } & PAGE & 2 OF 2 \\
\hline Kollector & $\begin{array}{l}\text { COMPANY CONTACT } \\
\text { WIDRIG, DL }\end{array}$ & $\begin{array}{l}\text { TELEPHONE NO. } \\
376-2858\end{array}$ & $\begin{array}{l}\text { PROJECT COORDINATOR } \\
\text { WIDRIG, DL }\end{array}$ & \multirow{2}{*}{$\begin{array}{l}\text { PRICE CODE } \\
\text { AIR QUALITY }\end{array}$} & $\mathbf{8 N}$ & \multirow{2}{*}{\multicolumn{2}{|c|}{$\begin{array}{c}\text { DATA } \\
\text { TURNAROUND } \\
45 \text { Days / } 45 \\
\text { Days }\end{array}$}} \\
\hline $\begin{array}{l}\text { SAMPLING LOCATION } \\
\text { C7018; EW2-010 }\end{array}$ & \multicolumn{2}{|c|}{$\begin{array}{l}\text { PROJECT DESIGNATION } \\
\text { 200-ZP-1 Remedial Action Wells Sampling and Analysis - Geochemical Mode }\end{array}$} & $\begin{array}{l}\text { SAF NO. } \\
\text { F09-012 }\end{array}$ & & $\square$ & & \\
\hline ICE CHEST NO. & $\begin{array}{l}\text { FIELD LOGBOOK NO. } \\
1+N F-N \cdot 585-3^{\text {PS3 }}\end{array}$ & $\begin{array}{l}\text { ACTUAL SAMPLE DEPTH } \\
217,7 \text { to } 2(8,7\end{array}$ & $\begin{array}{l}\text { COA } \\
\text { 300194ES10 }\end{array}$ & \multicolumn{4}{|c|}{$\begin{array}{l}\text { METHOD OF SHIPMENT } \\
\text { GOVERNMENT VEHICLE }\end{array}$} \\
\hline $\begin{array}{l}\text { SHIPPED TO } \\
\text { Environmental Sciences Laboratory }\end{array}$ & \multicolumn{2}{|l|}{$\begin{array}{l}\text { OFFSITE PROPERTY NO. } \\
\text { N/A }\end{array}$} & \multicolumn{3}{|c|}{$\begin{array}{l}\text { BILL OF LADING/AIR BILL NO. } \\
\text { N/A }\end{array}$} & & \\
\hline
\end{tabular}

\section{SPECIAL INSTRUCTIONS}

** The 200 Area S\&GRP Characterization and Monitoring Sampling and Analysis GKI applies to this SAF.

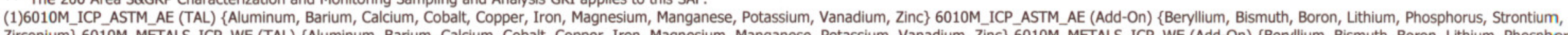

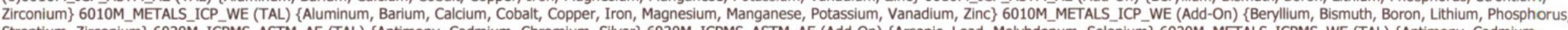

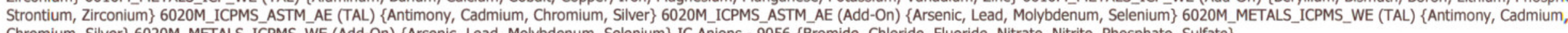
Chromium, Silver\}6020M_METALS_ICPMS_WE (Add-On) \{Arsenic, Lead, Molybdenum, Selenium\} IC Anions - 9056 \{Bromide, Chloride, Fluoride, Nitrate, Nitrite, Phosphate, Sulfate\}

(2)TOC - ASTME1915A \{Total organic carbon\} GAMMA_GS \{Cesium-137, Cobalt-60, Europium-152, Europium-154, Europium-155\}

(3)Moisture Content - D2216 \{Percent moisture (wet sample)\} 


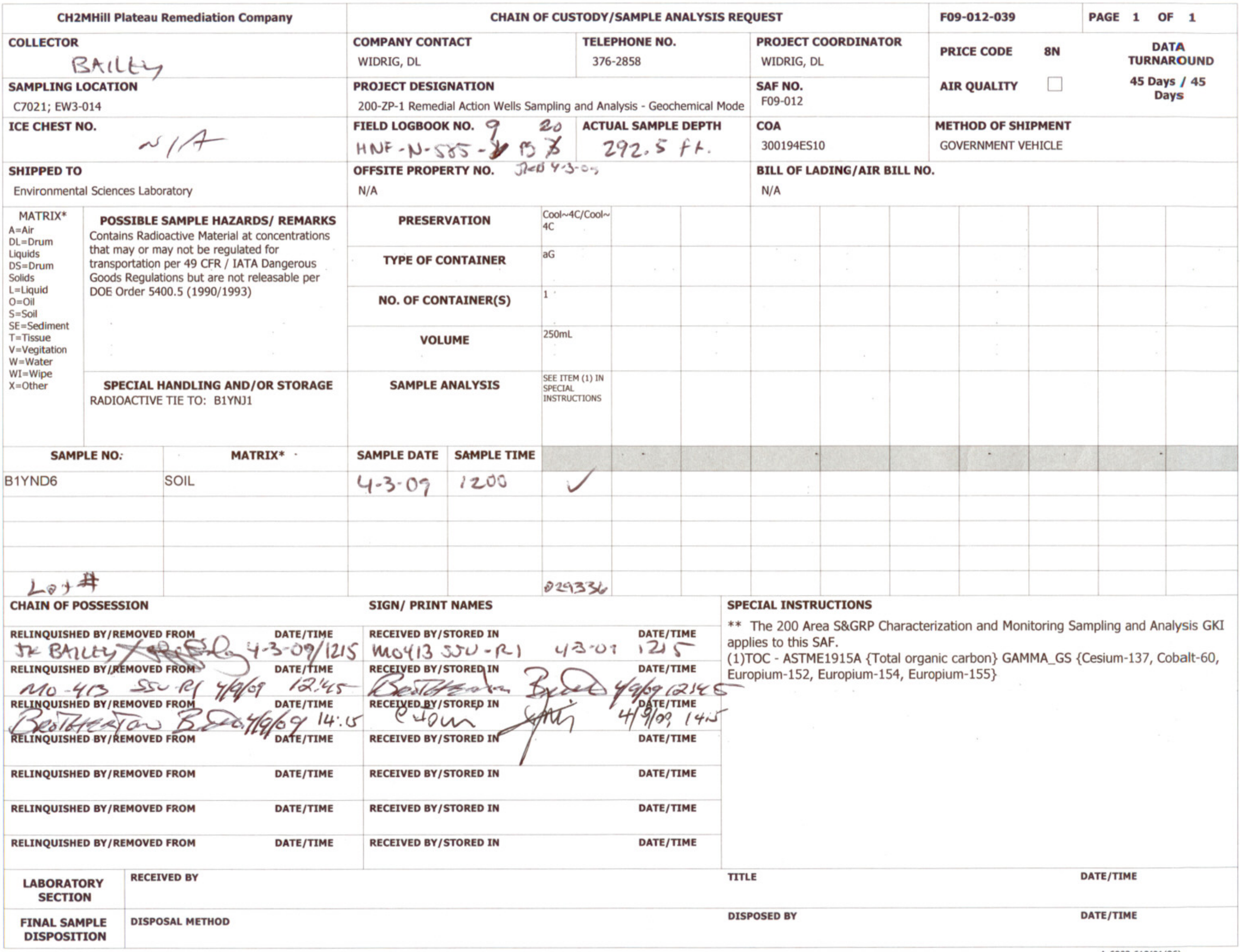




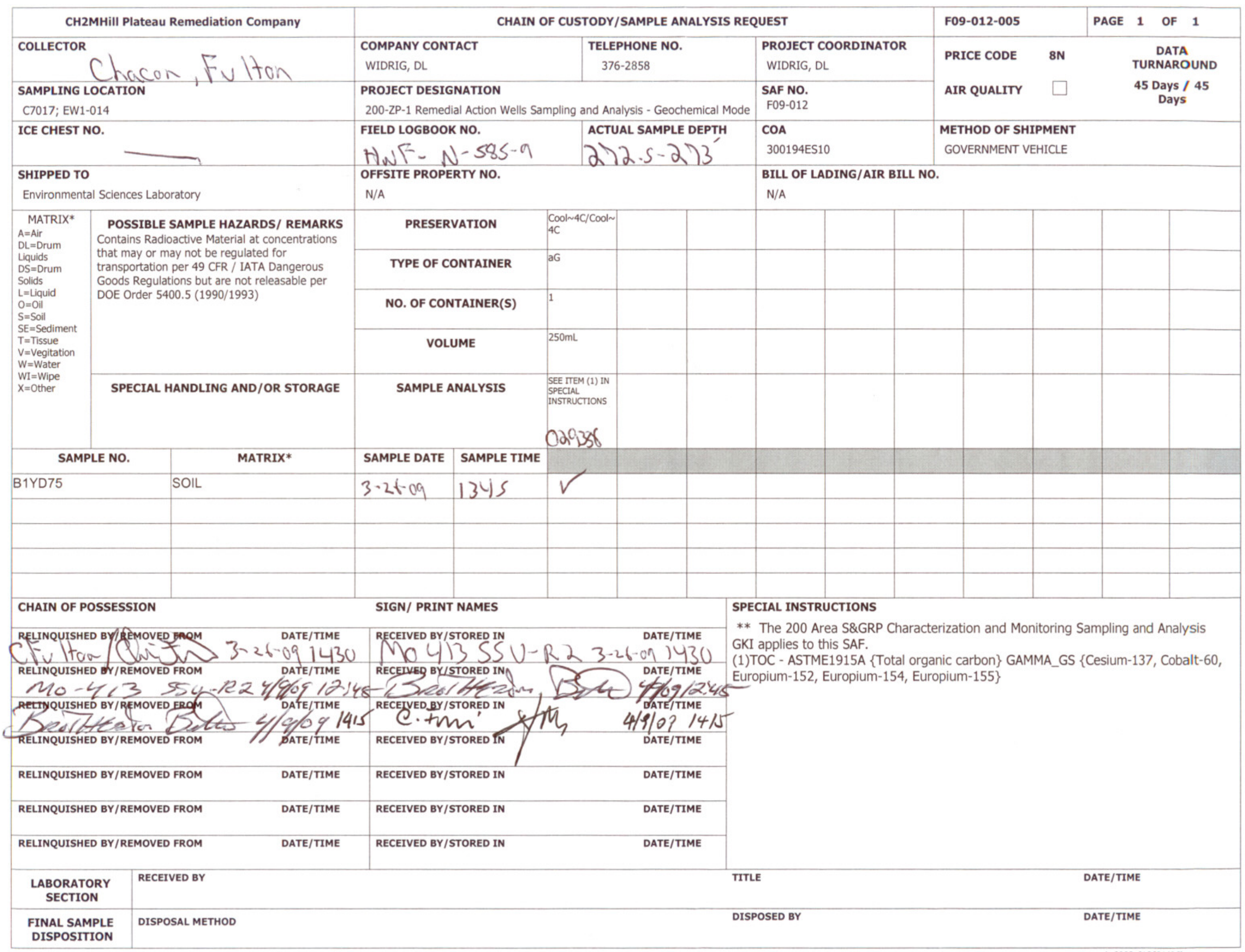




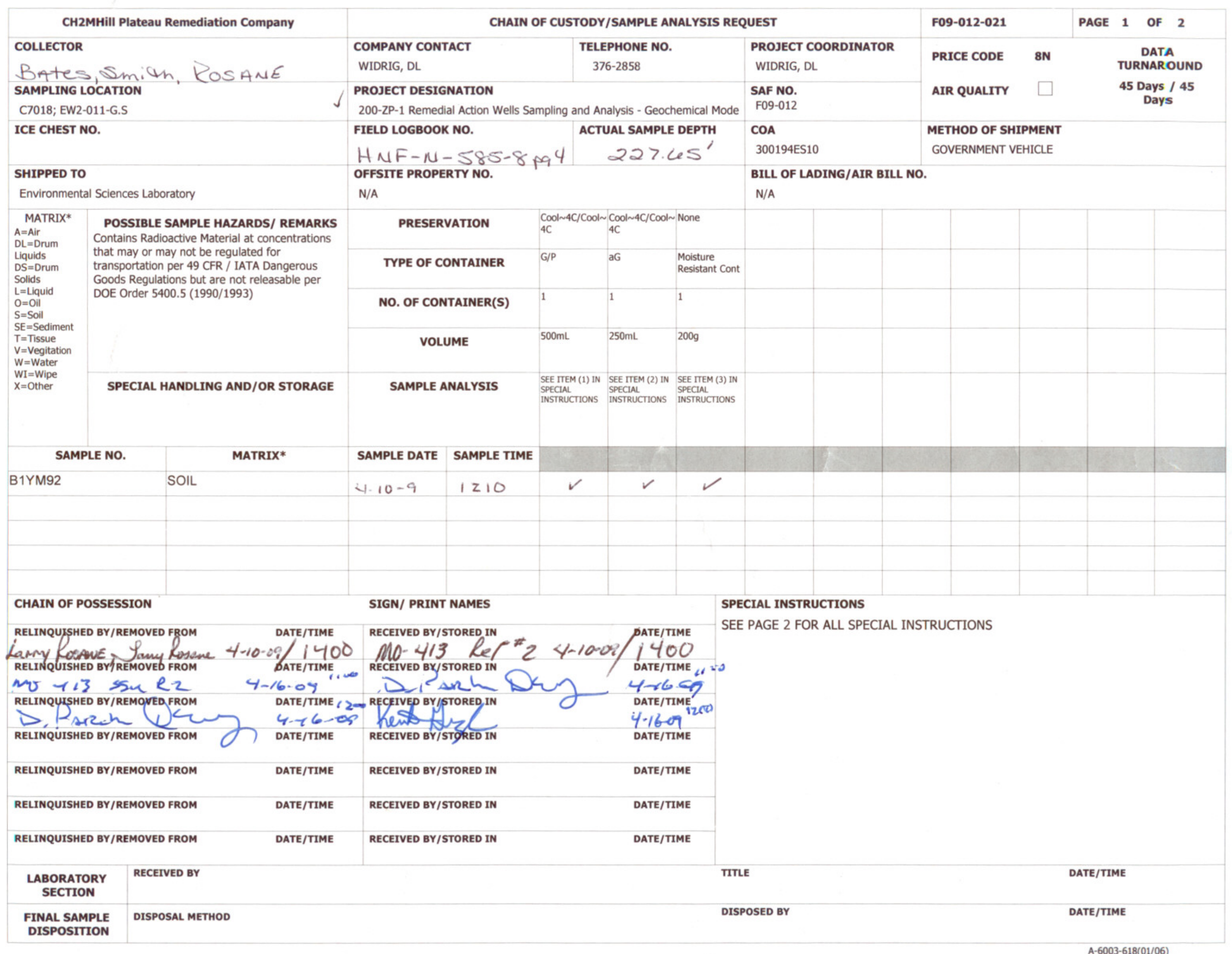




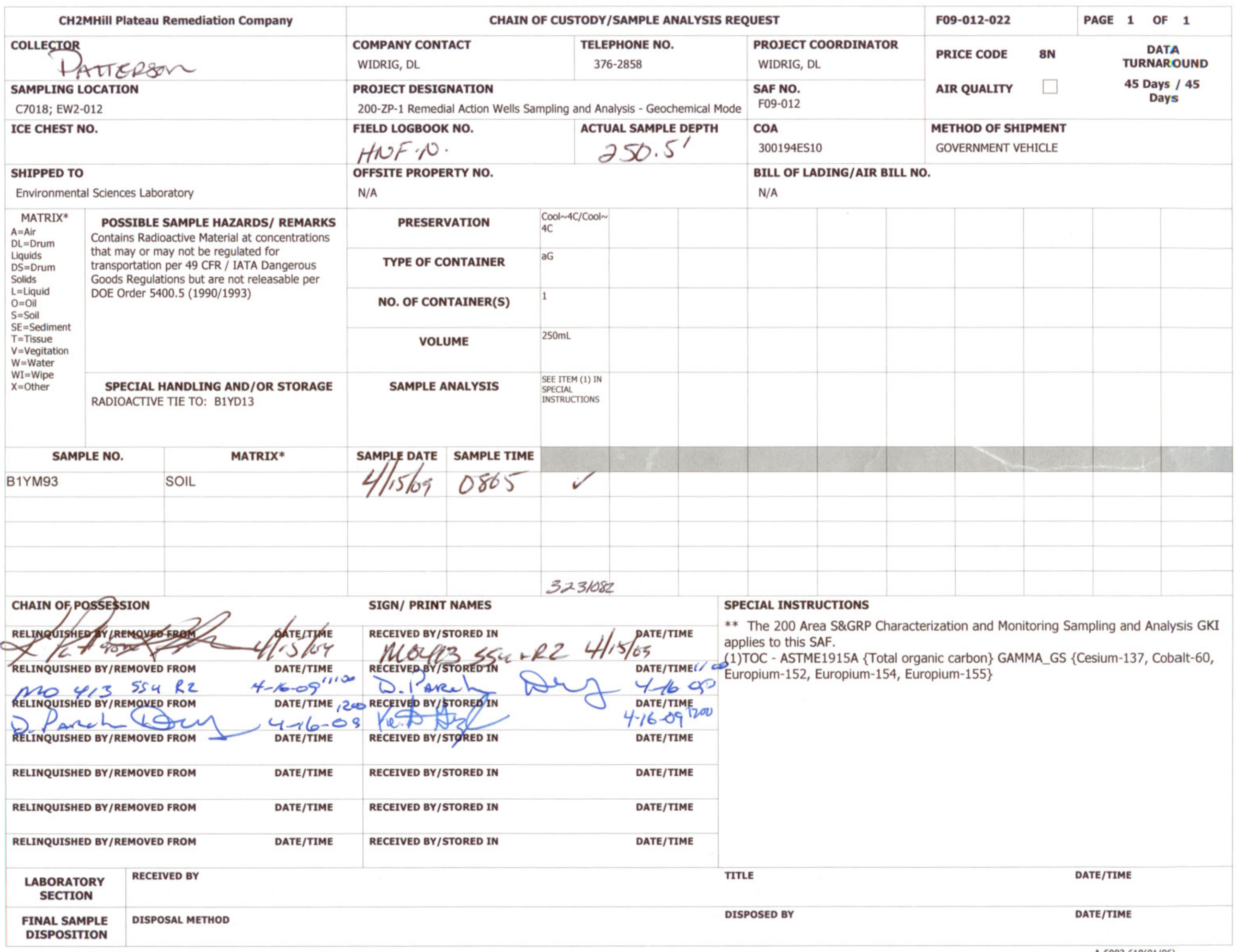




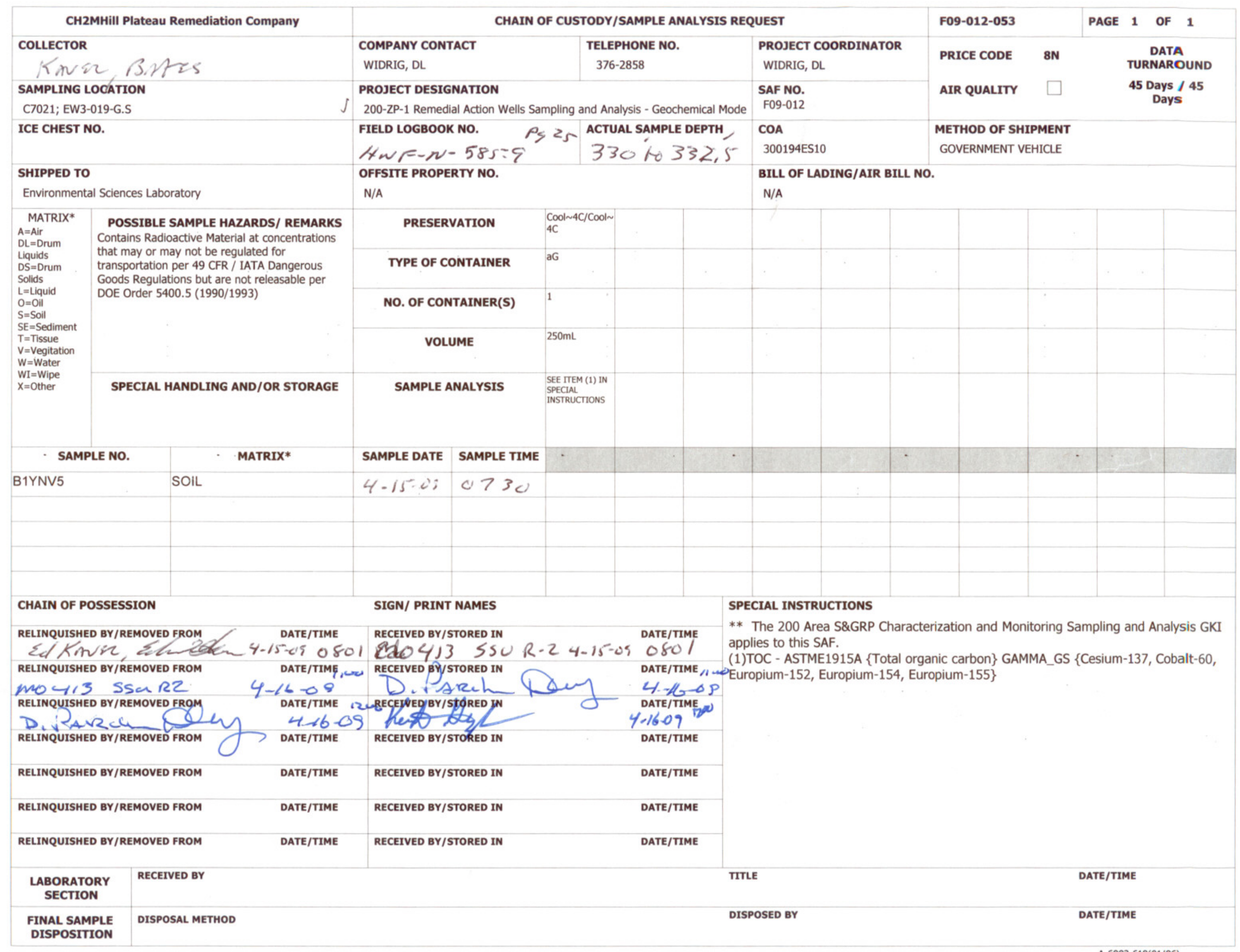




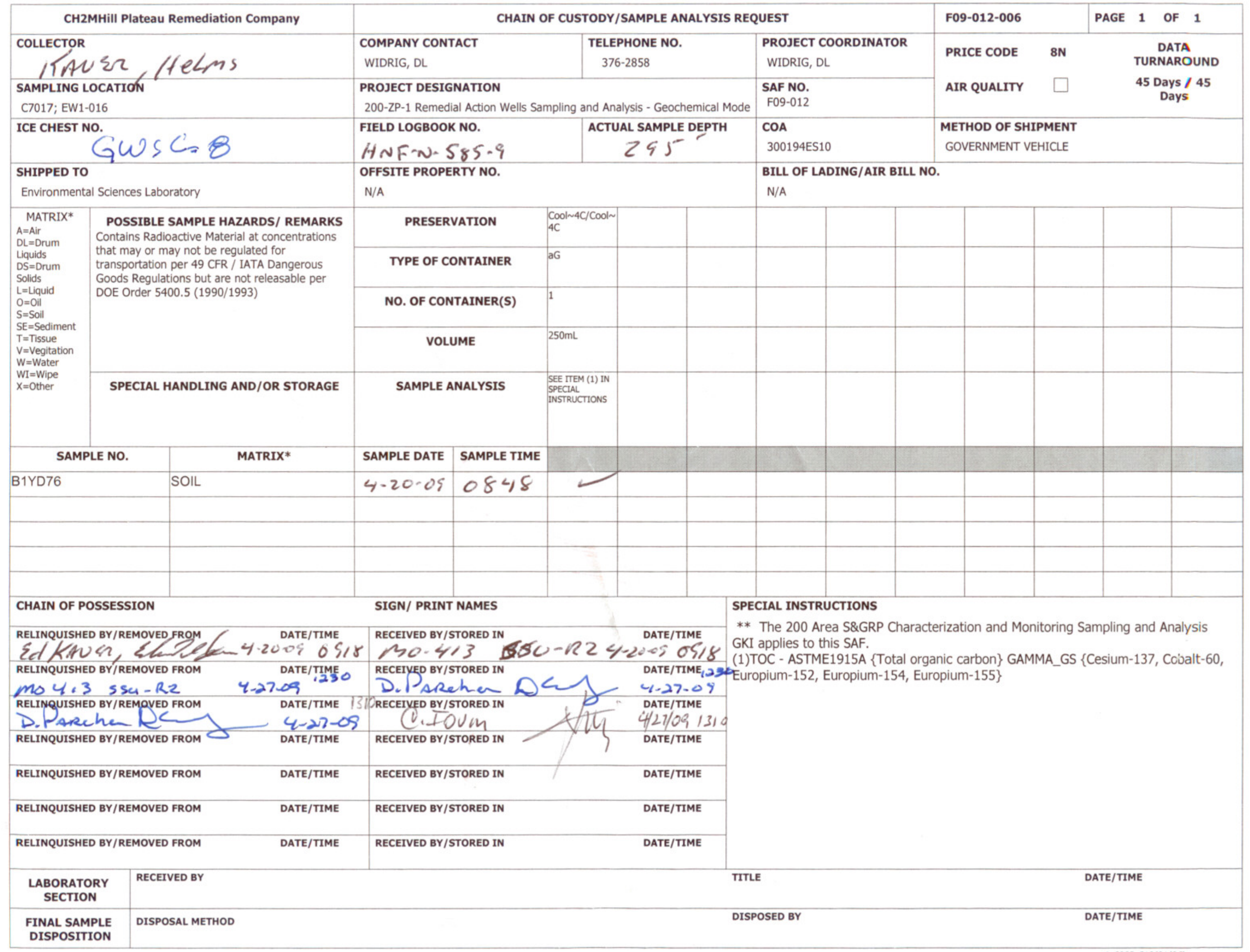




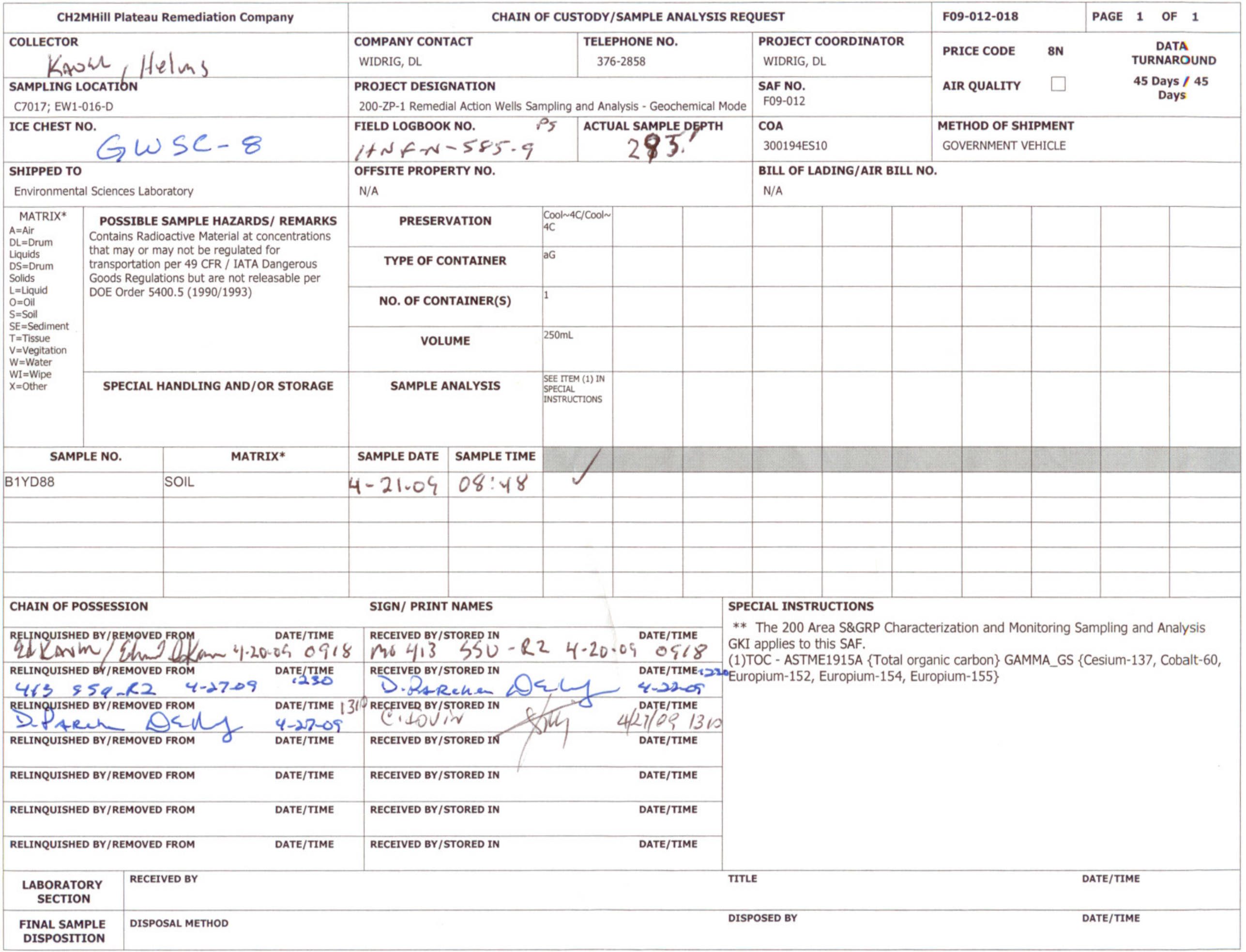




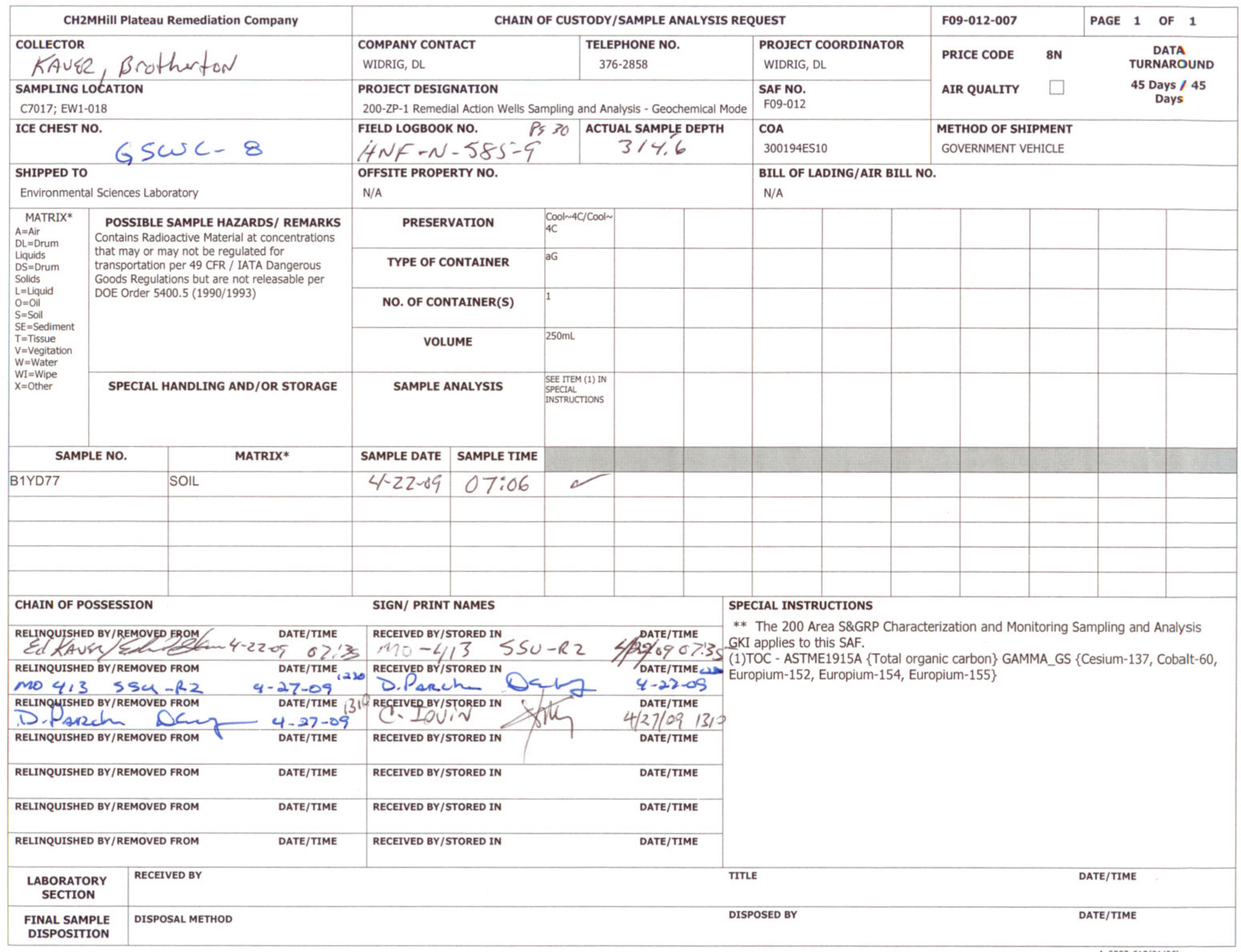




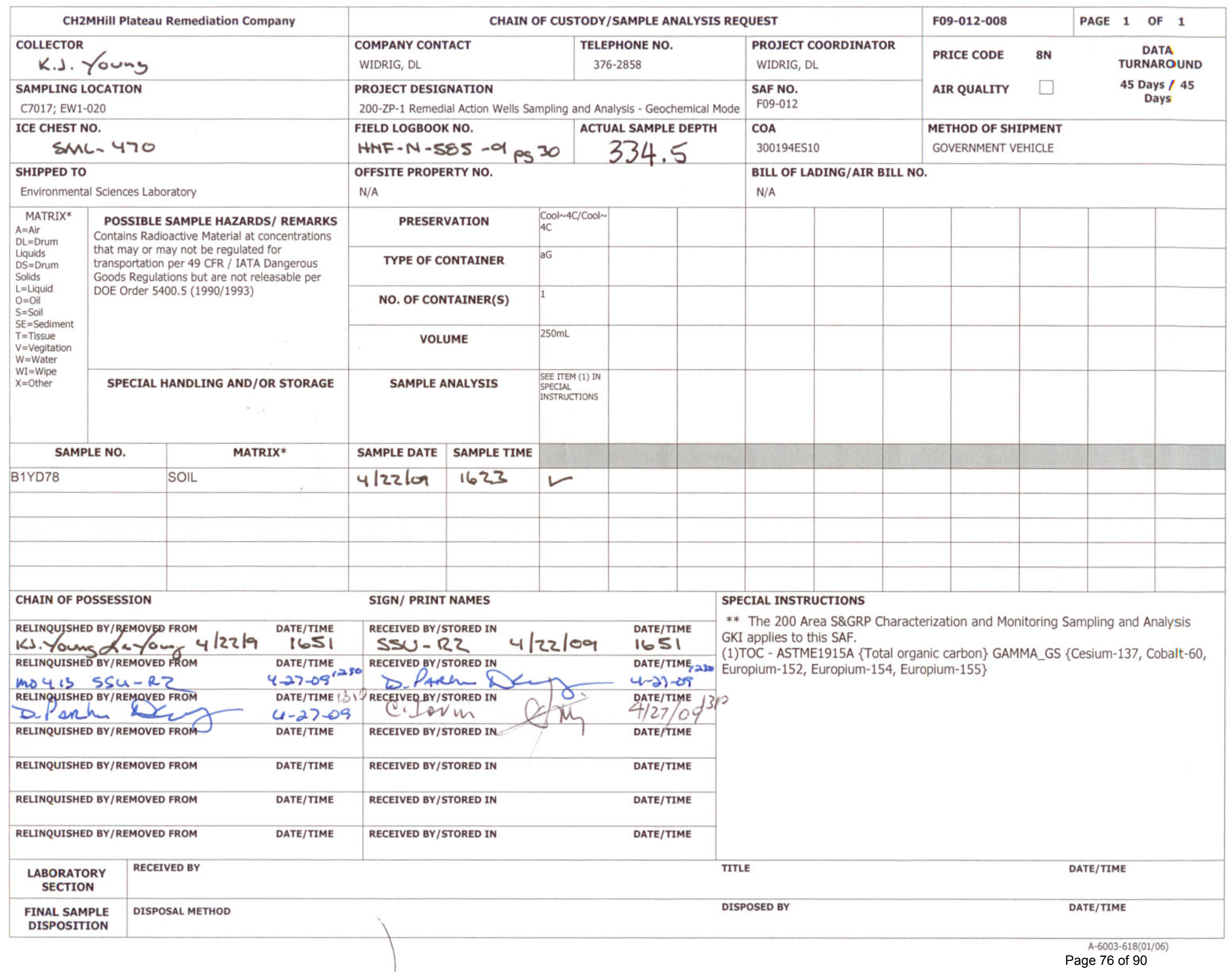




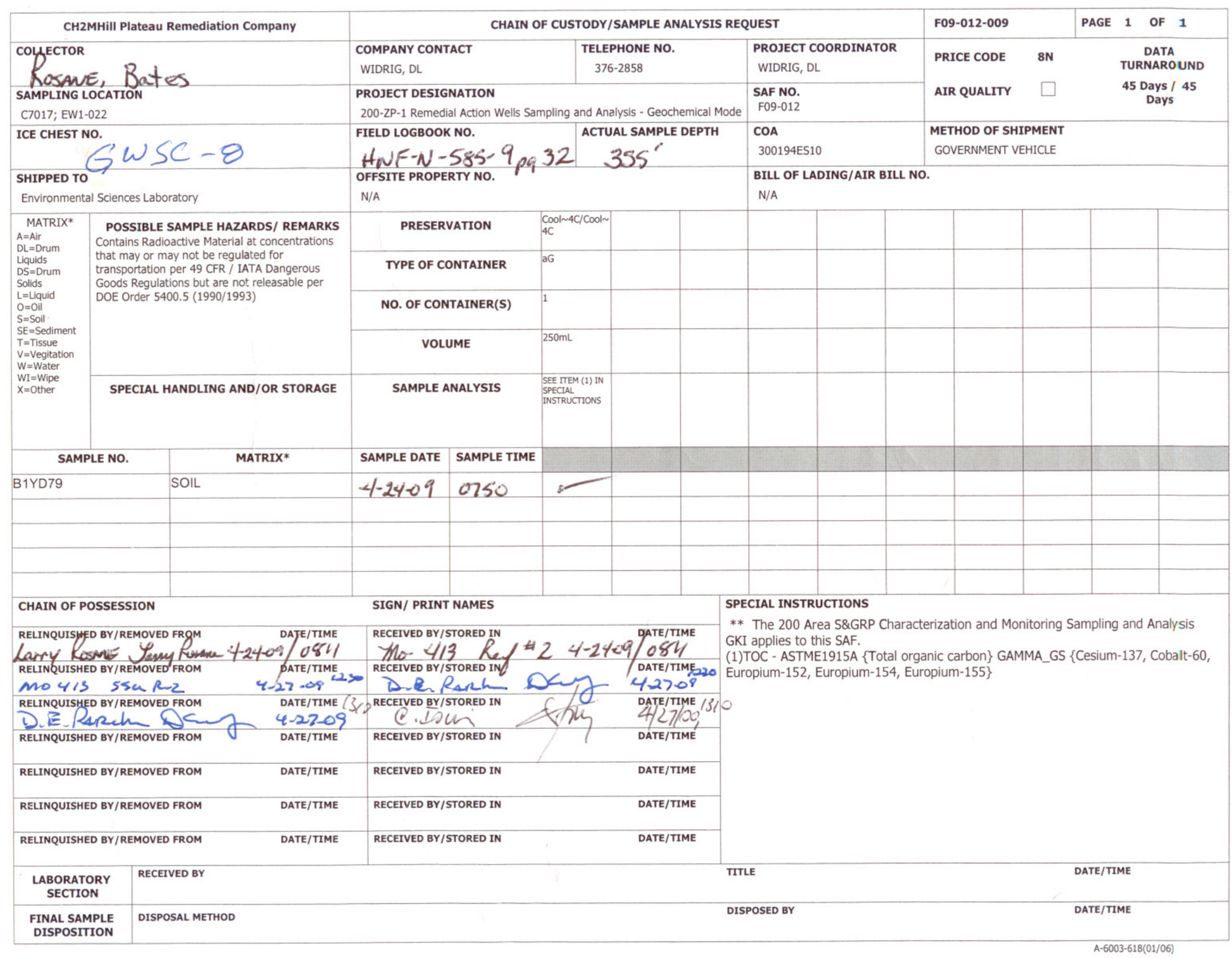




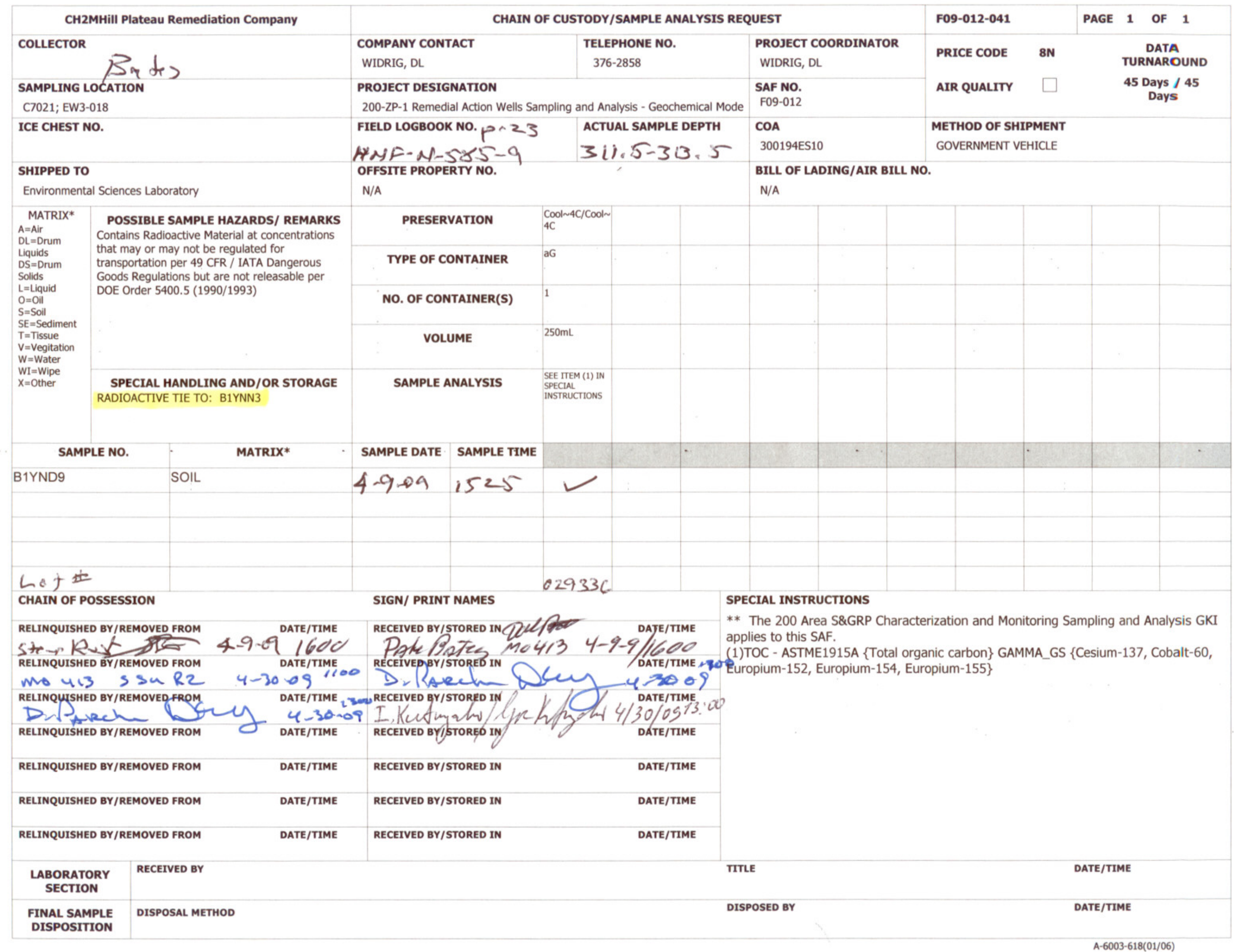




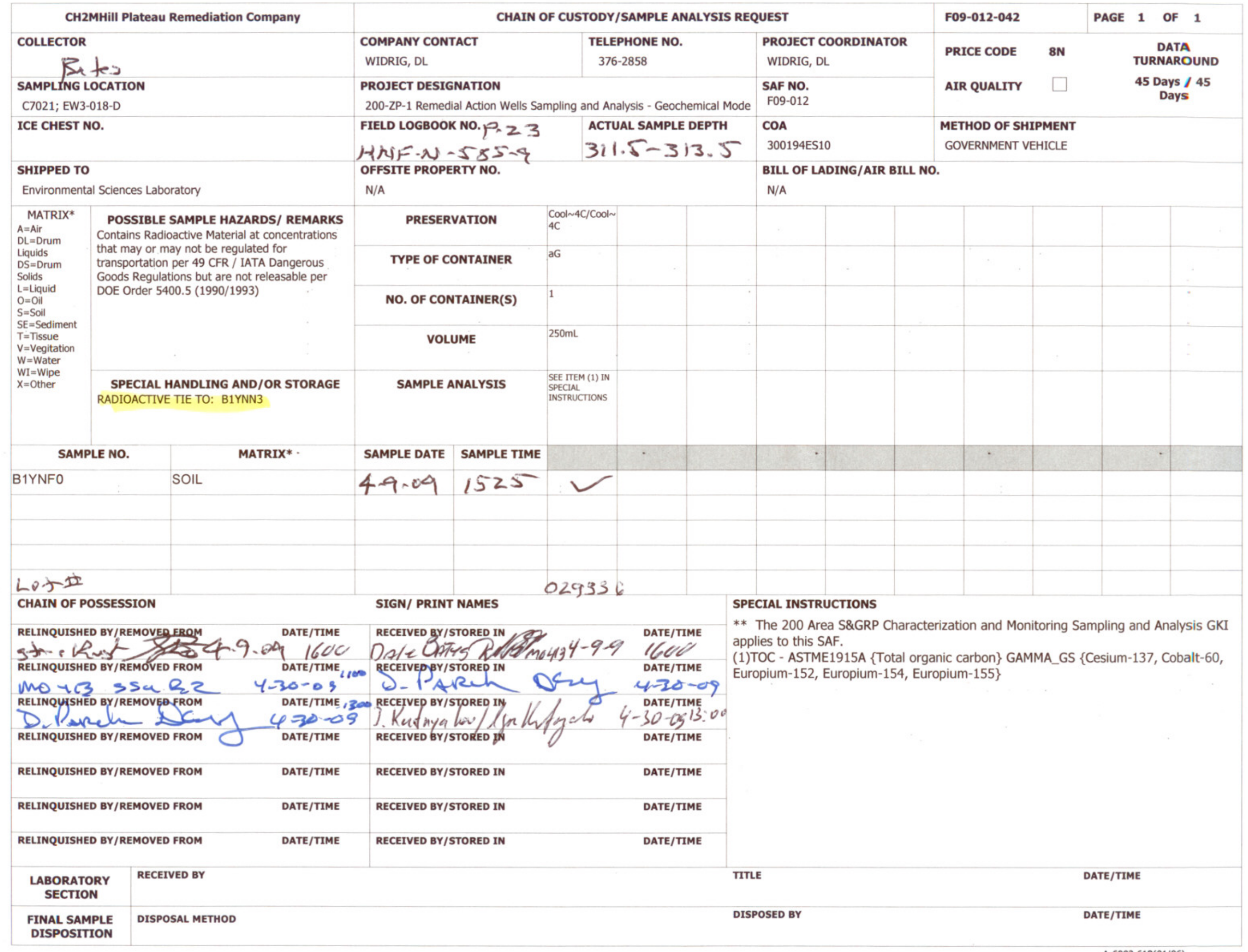


CH2MHill Plateau Remediation Company

\begin{tabular}{|c|c|}
\hline \multicolumn{2}{|c|}{ 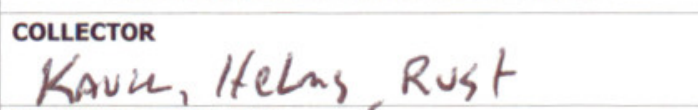 } \\
\hline \multicolumn{2}{|c|}{$\begin{array}{l}\text { SAMPLING LOCATION } \\
\text { C7021; EW3-020 }\end{array}$} \\
\hline \multicolumn{2}{|c|}{ ICE CHEST NO. } \\
\hline \multirow{2}{*}{\multicolumn{2}{|c|}{$\begin{array}{l}\text { SHIPPED TO } \\
\text { Environmental Sciences Laboratory }\end{array}$}} \\
\hline & \\
\hline \multirow[t]{2}{*}{$\begin{array}{l}\quad \text { MATRIX* } \\
\text { A=Air } \\
\text { DL=Drum } \\
\text { Liquids } \\
\text { DS }=\text { Drum } \\
\text { Solids } \\
\text { L=Liquid } \\
\text { O=Oil } \\
S=\text { Soil } \\
\text { SE=Sediment } \\
\mathrm{T}=\text { Tissue } \\
\mathrm{V}=\text { Vegitation } \\
\text { W=Water } \\
\text { WI=Wipe } \\
\mathrm{X}=\text { Other }\end{array}$} & $\begin{array}{l}\text { POSSIBLE SAMPLE HAZARDS/ REMARKS } \\
\text { Contains Radioactive Material at concentrations } \\
\text { that may or may not be regulated for } \\
\text { transportation per } 49 \text { CFR / IATA Dangerous } \\
\text { Goods Regulations but are not releasable per } \\
\text { DOE Order } 5400.5(1990 / 1993)\end{array}$ \\
\hline & $\begin{array}{l}\text { SPECIAL HANDLING AND/OR STORAGE } \\
\text { RADIOACTIVE TIE TO: B1YNN3 }\end{array}$ \\
\hline
\end{tabular}

\section{CHAIN OF CUSTODY/SAMPLE ANALYSIS REQUEST}

COMPANY CONTACT

WIDRIG, DL

\section{PROJECT DESIGNATION}

200-ZP-1 Remedial Action Wells Sampling and Analysis - Geochemical Mode FIELD LOGBOOK NO.
I $N$ F F -585.4 OFFSITE PROPERTY NO.

N/A

PS $2 \checkmark$ ACTUAL SAMPLE DEPTH

$$
\text { PRESERVATION }
$$

TYPE OF CONTAINER

NO. OF CONTAINER(S)

\begin{tabular}{|c|l|}
\hline VOLUME & $250 \mathrm{~mL}$ \\
\hline SAMPLE ANALYSIS & $\begin{array}{l}\text { SEE TEM (1) IN } \\
\text { SPECIAL } \\
\text { INSTRUCTIONS }\end{array}$ \\
\hline
\end{tabular}

SAMPLE DATE SAMPLE TIME

\section{SAMPLE NO.}

MATRIX*

\begin{tabular}{|l|l|}
\hline B1YNF1 SOIL \\
\hline
\end{tabular}

\section{RELINQUISHED BY/REMOVED FRPM/DATE/TIME}

\section{SIGN/ PRINT NAMES}

RECEIVED BY/STORED IN
F09-012-043

\section{PROJECT COORDINATOR}

WIDRIG, DL

SAF NO.

F09-012

COA

N/A

PRICE CODE
300194ES10

BILL OF LADING/AIR BILL NO.

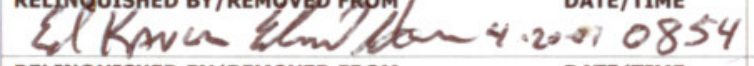

DATE/TIME LOD RECEIVER BY/STORED IN

RECEIVEP BY/STORED IN $L=R 2 \cdot 4.20 .050854$

D. Parch

RELINQUISHED BY/REMOVED FROM

WO $4.3>S 4 R 2$

D. Parehn

RELINQUISHED BY/REMOVED FROM

RELINQUISHED BY/REMOVED FROM

RELINQUISHED BY/REMOVED FROM

RELINQUISHED BY/REMOVED FROM

4. 30 -OS DATE/TIME, 3 ORECEIVED BY/STORED/IN/

4. 40.09

DATE/TIME RECEIVED BY/STORED WN

RECEIVED BY/STORED IN

DATE/TIME

RECEIVED BY/STORED IN

RECEIVED BY/STORED IN

DATE/TIME

DATE/TIME

DATE/TIME

DATE/TIME log

\begin{tabular}{|l|}
\hline $\begin{array}{l}\text { CoOI } 4 C / C O O I ~ \\
4 C\end{array}$ \\
\hline aG \\
\hline 1 \\
\hline $250 \mathrm{~mL}$ \\
\hline $\begin{array}{l}\text { SEE ITEM (1) IN } \\
\text { PEECAL } \\
\text { INSTRUCTIONS }\end{array}$ \\
\hline
\end{tabular}

PAGE 1 OF 1

DATA

AIR QUALITY $\square \quad 45$ Days / 45

Days

\section{METHOD OF SHIPMENT}

GOVERNMENT VEHICLE

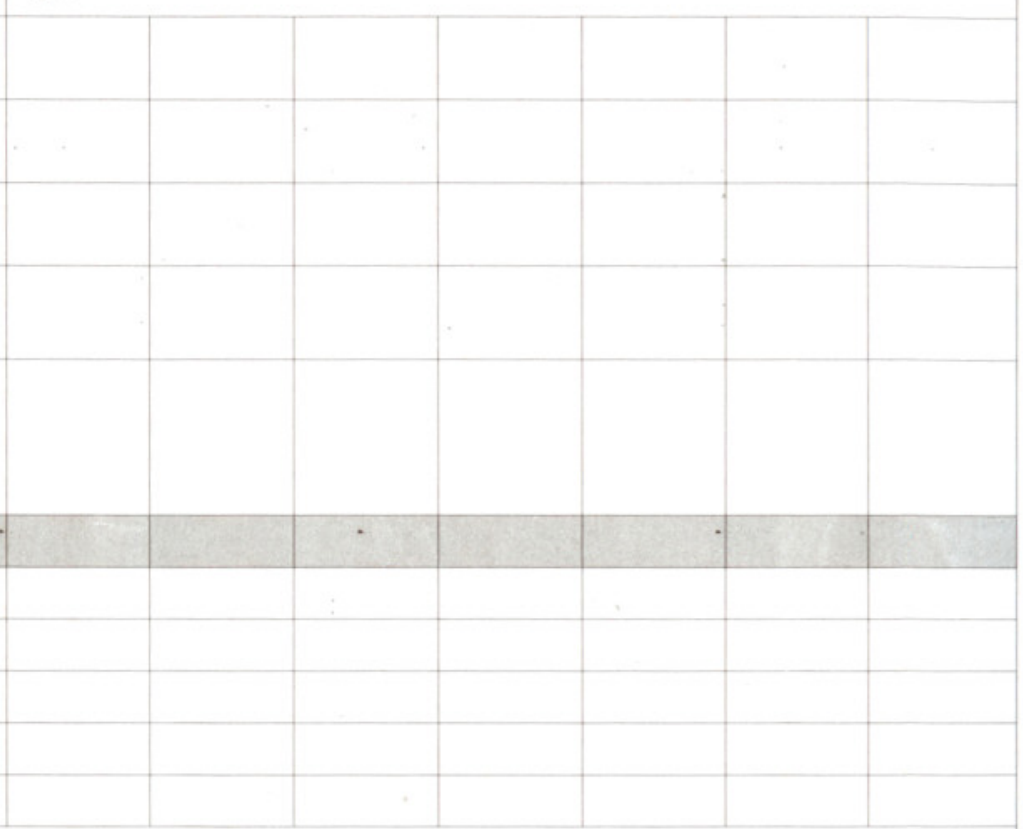

SPECIAL INSTRUCTIONS

** The 200 Area S\&GRP Characterization and Monitoring Sampling and Analysis GKI applies to this SAF.

(1)TOC - ASTME1915A \{Total organic carbon\} GAMMA_GS \{Cesium-137, Cobalt-60,

DATE/TIME "1U E Europium-152, Europium-154, Europium-155\}

4. DATE/TIME

DATE/TIME

\begin{tabular}{|c|c|c|c|}
\hline $\begin{array}{l}\text { LABORATORY } \\
\text { SECTION }\end{array}$ & RECEIVED BY & TITLE & DATE/TIME \\
\hline $\begin{array}{l}\text { FINAL SAMPLE } \\
\text { DISPOSITION }\end{array}$ & DISPOSAL METHOD & DISPOSED BY & DATE/TIME \\
\hline
\end{tabular}




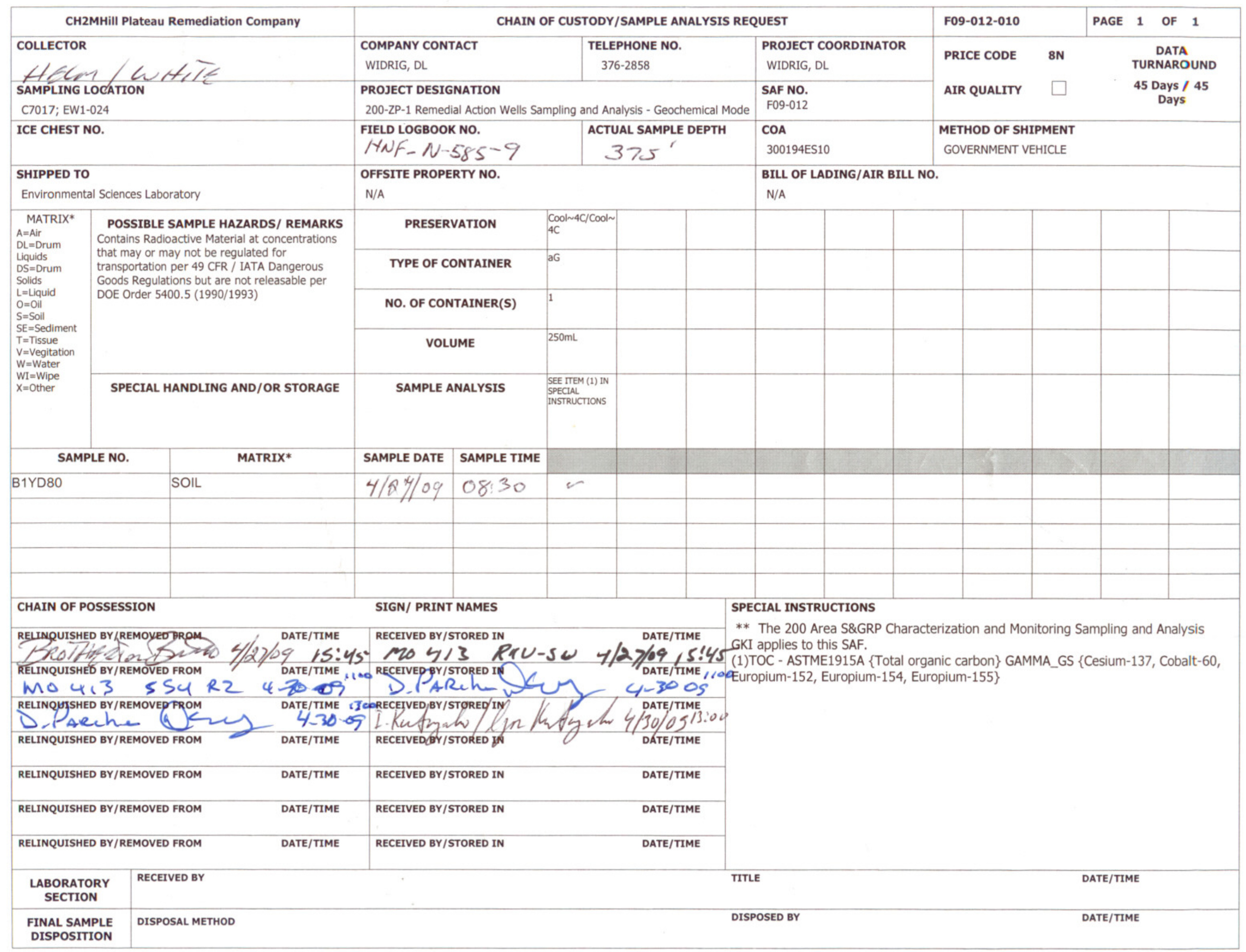




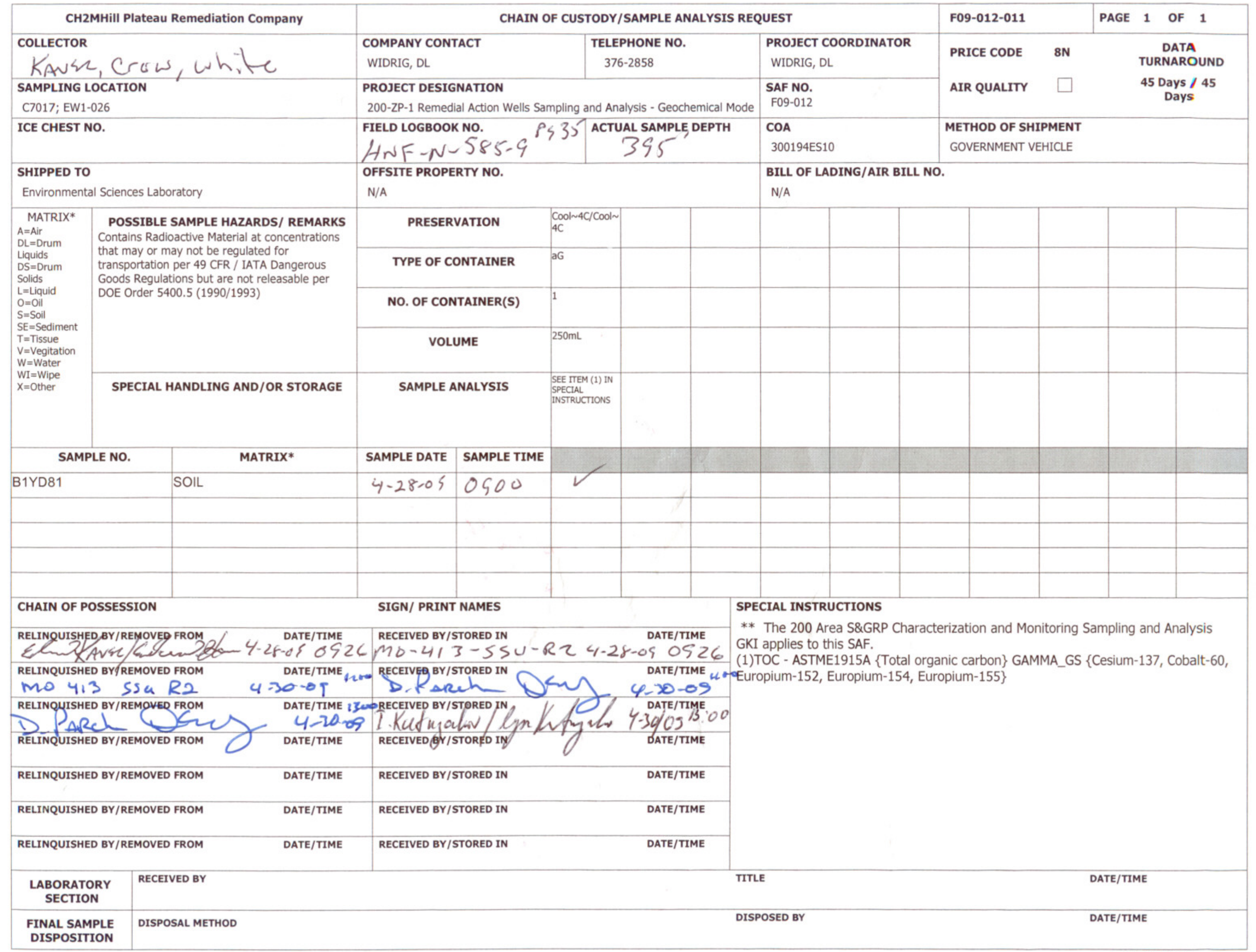


CH2MHill Plateau Remediation Company

\section{COLLECTOR}

\section{intite}

SAMPLING LOCATION

C7017; EW1-028

ICE CHEST NO.

\section{SHIPPED TO}

Environmental Sciences Laboratory

\begin{tabular}{|c|c|}
\hline \multirow[t]{2}{*}{$\begin{array}{l}\quad \text { MATRIX* } \\
\text { A=Air } \\
\text { DL=Drum } \\
\text { Liquids } \\
\text { DS=Drum } \\
\text { Solids } \\
\text { L=Liquid } \\
O=\text { Oil } \\
\text { S=Soil } \\
\text { SE=Sediment } \\
\text { T=Tissue } \\
V=\text { Vegitation } \\
\text { W=Water } \\
\text { WI=Wipe }\end{array}$} & $\begin{array}{l}\text { POSSIBLE SAMPLE HAZARDS/ REMARKS } \\
\text { Contains Radioactive Material at concentrations } \\
\text { that may or may not be regulated for } \\
\text { transportation per } 49 \text { CFR / IATA Dangerous } \\
\text { Goods Regulations but are not releasable per } \\
\text { DOE Order } 5400.5 \text { (1990/1993) }\end{array}$ \\
\hline & SPECIAL HANDLING AND/OR STORAGE \\
\hline
\end{tabular}

\begin{tabular}{|l|l|l|}
\hline COMPANY CONTACT & TELEPHONE NO. & PROJECT COORDINATOR
\end{tabular}

CHAIN OF CUSTODY/SAMPLE ANALYSIS REQUEST

WIDRIG, DL

\section{PROJECT DESIGNATION}

$376-2858$

200-ZP-1 Remedial Action Wells Sampling and Analysis - Geochemical Mode FIELD LOGBOOK NO. BS $\quad$ ACTUAL SAMPLE DEPTH ItNF-N-585-9 $36 \quad 414$ OFFSITE PROPERTY NO.

$\mathrm{N} / \mathrm{A}$
SAF NO.

F09-012

COA

300194ES10

BILL OF LADING/AIR BILL NO.

\section{WIDRIG, DL}

\begin{tabular}{|cc|cc|}
\hline F09-012-012 & PAGE 1 OF 1 \\
PRICE CODE & $8 \mathrm{~N}$ & $\begin{array}{c}\text { DATA } \\
\text { TURNAROUND } \\
\text { AIR QUALITY }\end{array}$ & $\begin{array}{c}\text { 45 Days / 45 } \\
\text { Days }\end{array}$ \\
\hline
\end{tabular}

METHOD OF SHIPMENT

GOVERNMENT VEHICLE

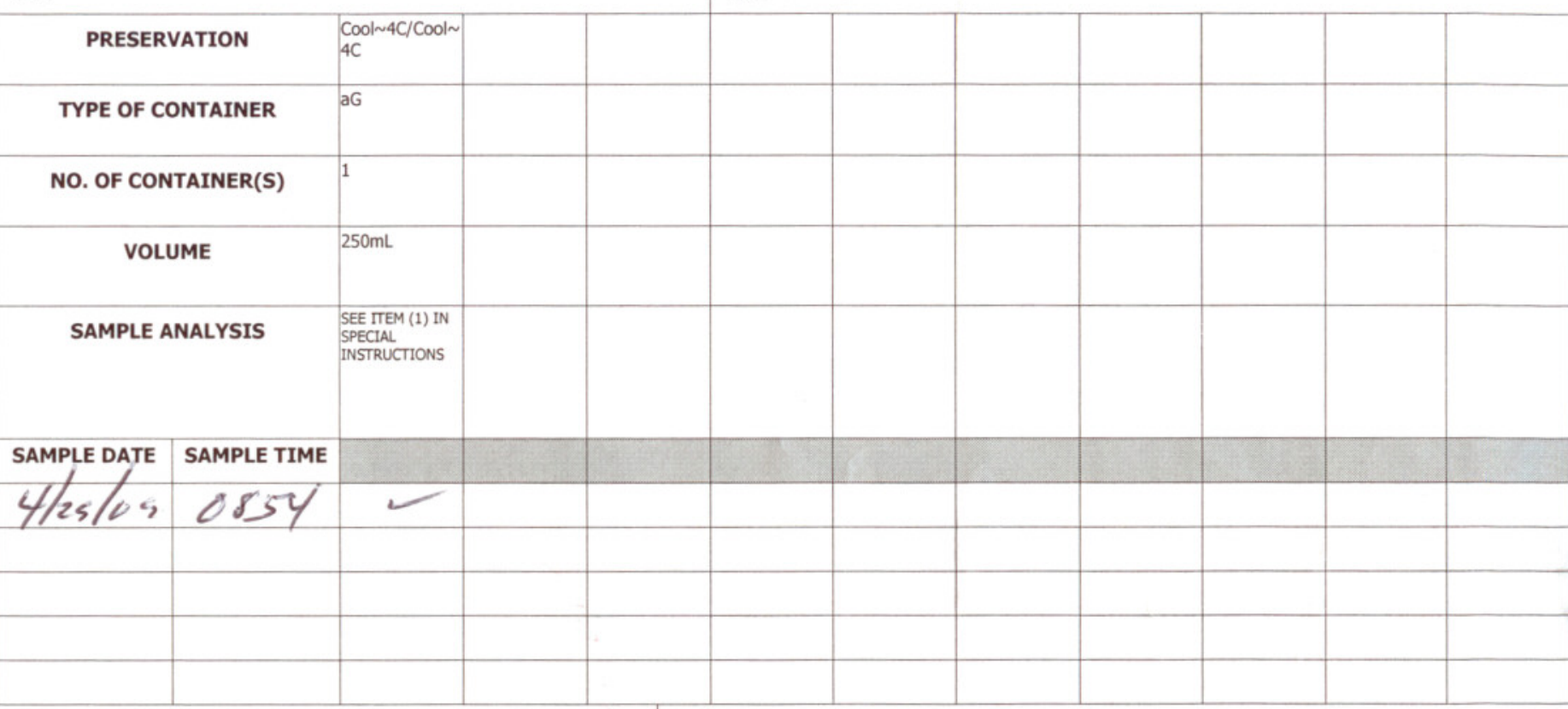

\section{SIGN/ PRINT NAMES}

RECEIVED BY/STORED IN

\section{CHAIN OF POSSESSION}

RELINGUISHEO BY/REMOVED FROM Xlarnan $\rightarrow$ RELINQUISHED BY/REMOVED FROM mo 413 ss RELINQUISHED BY/REMOVED FROM

$\rightarrow J$ Prchen h RELINQUISHED BY/REMOVED FROM RELINOUISHED BY/REMOVED FROM RELINQUISHED BY/REMOVED FROM RELINQUISHED BY/REMOVED FROM DATE/TIME $25 /{ }_{2} 09$ MO- 3 S RECEIVED-BY/STORED IN Y 20 - OS

DATE/TIMEF 300 RECEIVED,BY/STORED IN/ DATE/TIME, SURZ Y/2S/ DATE/TIME

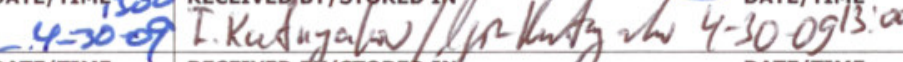
DATE/TIME RECEIVED BK/STORED IN DATE/TIME

\section{DATE/TIME}

RECEIVED BY/STORED IN

DATE/TIME

DATE/TIME

RECEIVED BY/STORED IN

DATE/TIME

RECEIVED BY/STORED IN

DATE/TIME

\section{** The 200 Area S\&GRP Characterization and Monitoring Sampling and Analysis}

S O 33 GKI applies to this SAF. DATE/TIME nO Europium-152, Europium-154, Europium-155

\begin{tabular}{|c|c|c|c|}
\hline $\begin{array}{l}\text { LABORATORY } \\
\text { SECTION }\end{array}$ & RECEIVED BY & TITLE & DATE/TIME \\
\hline $\begin{array}{l}\text { FINAL SAMPLE } \\
\text { DISPOSITION }\end{array}$ & DISPOSAL METHOD & DISPOSED BY & DATE/TIME \\
\hline
\end{tabular}




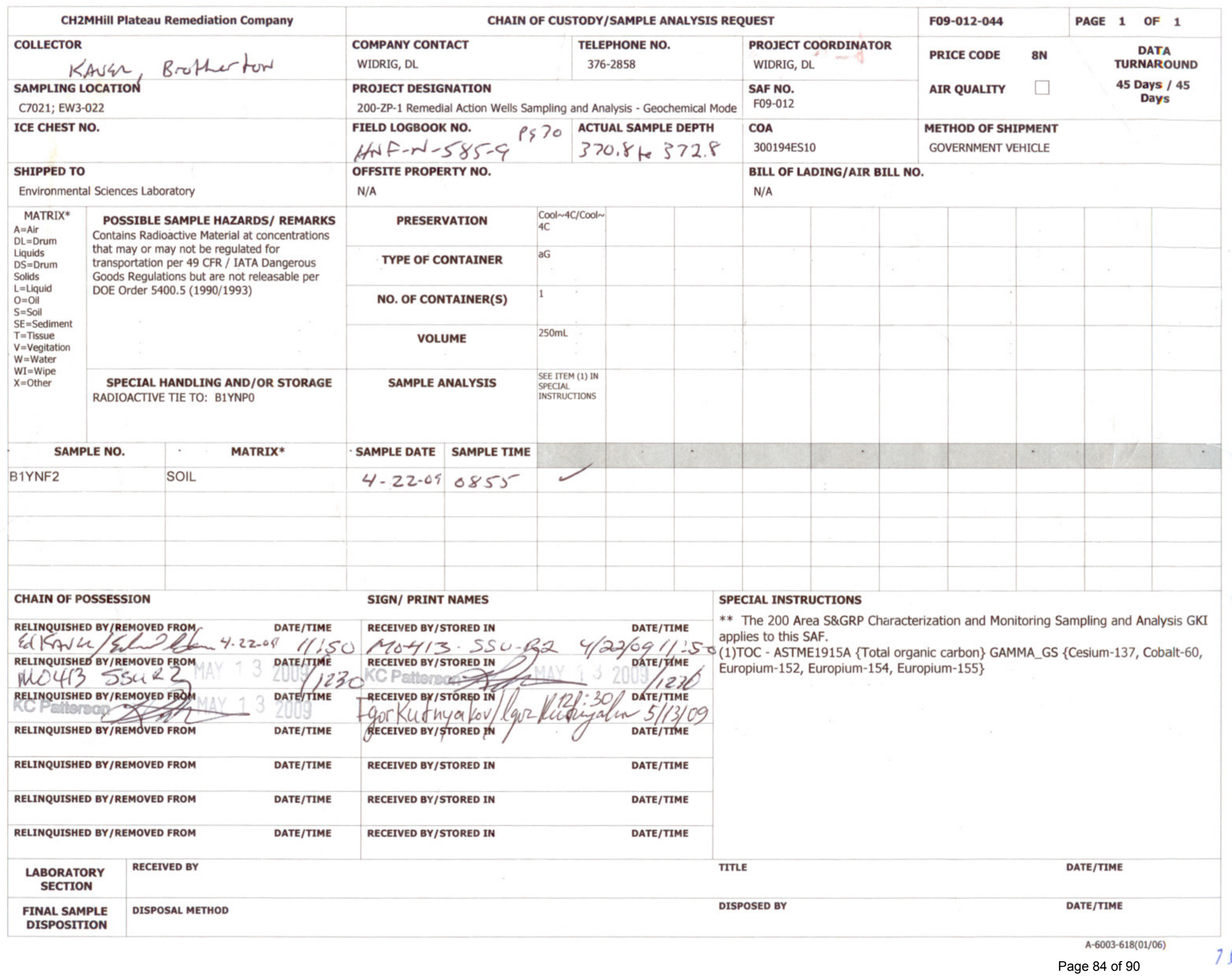




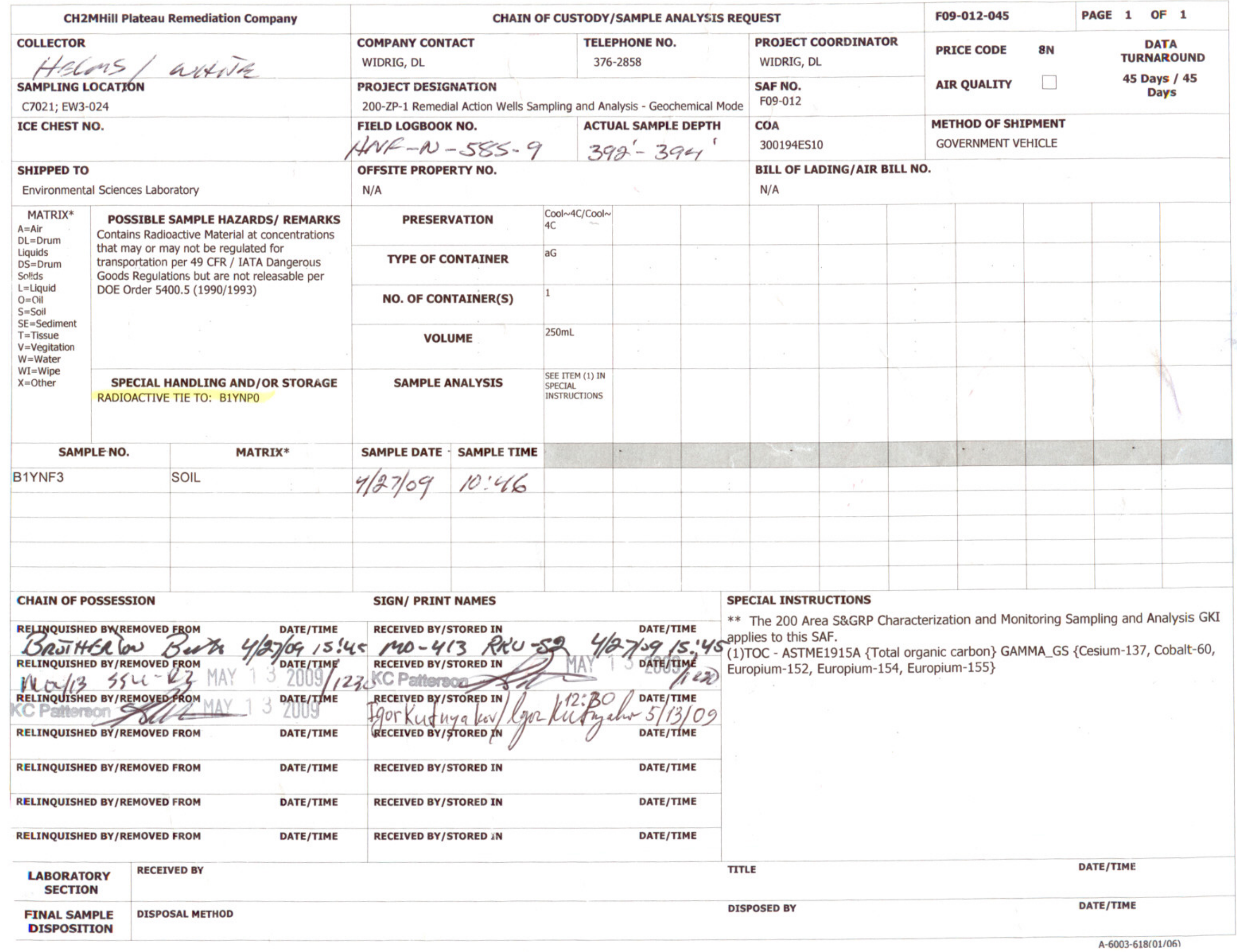




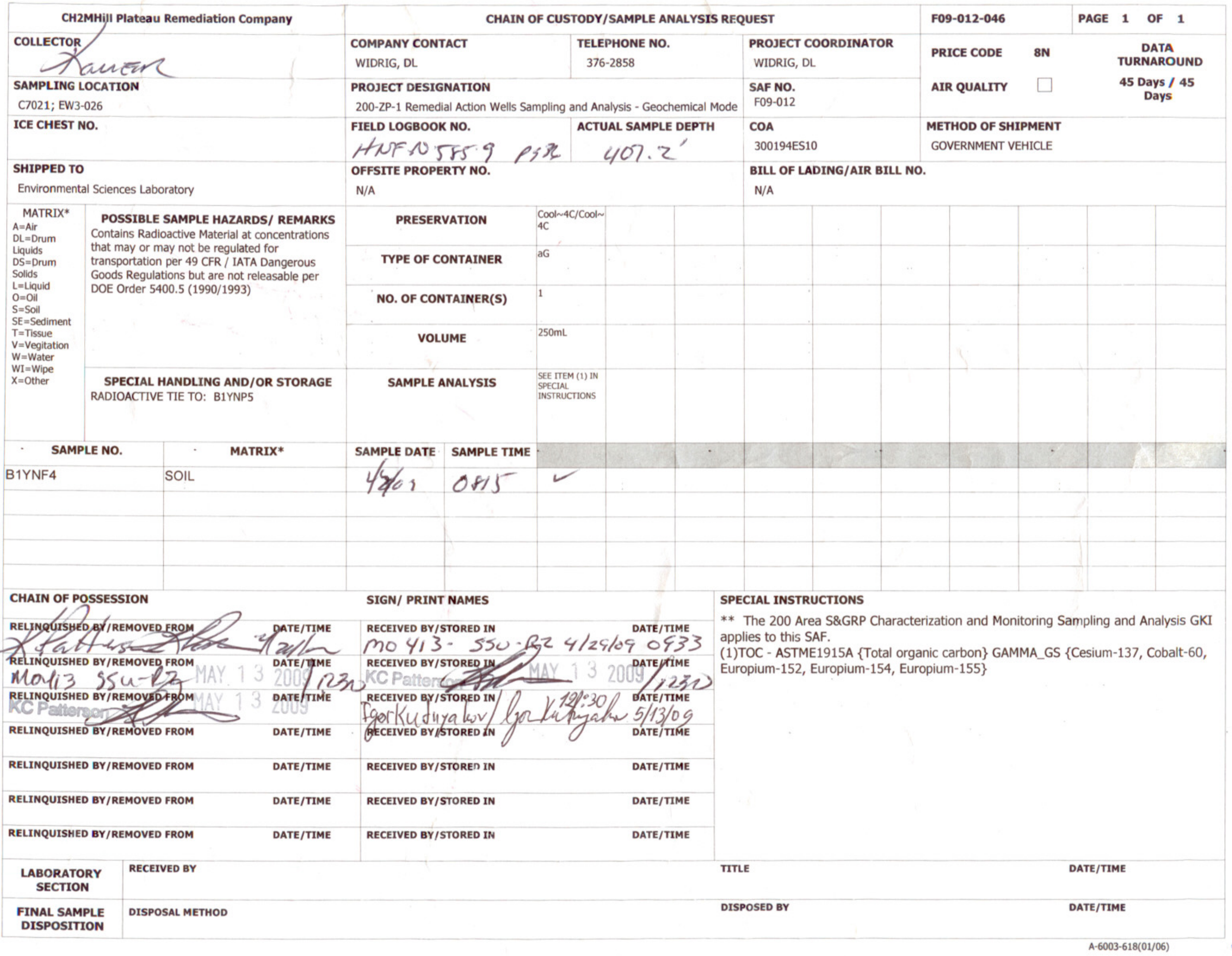

Page 86 of 90 
CH2MHill Plateau Remediation Company

\section{COLLECTOR \\ Losane}

SAMPLING LOCATION

C7021; EW3-028

ICE CHEST NO.

\section{SHIPPED TO}

Environmental Sciences Laboratory

\section{MATRIX* POSSIBLE SAMPLE HAZARDS/ REMARKS $A=A i r \quad$ Contains Radioactive Material at concentrations \begin{tabular}{l|l}
$\mathrm{DL}=$ Drum \\
Liquids that may or may not be regulated for
\end{tabular} \\ DS=Drum transportation per 49 CFR / IATA Dangerous \\ Solids Goods Regulations but are not releasable per \\ $\mathrm{L}=$ Liquid
$\mathrm{O}=0 \mathrm{il}$ \\ $\mathrm{SE}=$ Sedimen \\ $T=T$ issue
$V=$ Vegitation \\ $\mathrm{W}=$ Water \\ WI $=$ Wipe \\ $\mathrm{X}=$ Other \\ SPECIAL HANDLING AND/OR STORAGE RADIOACTIVE TIE TO: B1YNP5}

\section{SAMPLE NO.}

B1YNF5

\begin{tabular}{|l|l|}
\hline B1YNF5 SOIL \\
\hline & \\
\hline & \\
\hline CHAIN OF POSSESSION & \\
\hline
\end{tabular}

\section{RELINQUISHED BY/REMOVED EROM}

RELINQUISHED BY/REMOVED FROM
Larry RCSAUE, Jimy Lodane $5-1-09 / 1220$
RELINOUISHED BY/REMOVED FROM

Netl ssu-nz

RELINQUISHED BY/REMQIEDFROM

RELINQUISHED BY/REMOVED FROM

1220

DATE/TIME 1230 DATE/TIME

RELINQUISHED BY/REMOVED FROM DATE/TIME

RELINQUISHED BY/REMOVED FROM DATE/TIME

RELINQUISHED BY/REMOVED FROM

DATE/TIME

DATE/TIME

CHAIN OF CUSTODY/SAMPLE ANALYSIS REQUEST

COMPANY CONTACT

WIDRIG, DL

TELEPHONE NO.

376-2858

PROJECT DESIGNATION

200-ZP-1 Remedial Action Wells Sampling and Analysis - Geochemical Mode FIELD LOGBOOK NO.

ACTUAL SAMPLE DEPTH

HNF-N.585-9 ag $37 \quad 432 \div 434$ OFFSITE PROPERTY NO.

N/A

PRESERVATION

TYPE OF CONTAINER

NO. OF CONTAINER(S)

VOLUME

SAMPLE ANALYSIS

Cool 4C/Cool

1

SEE TEM (1) IN SPECIAL
INSTRUCTIONS

SAMPLE DATE SAMPLE TIME

$5-1-09 / 140$

\section{SIGN/ PRINT NAMES}

RECEIVED BY/STORED IN RECEIVED BY/STORED IN
MO- 413 Ref 3 PATE/TIME
RECEIVED BY/STORED IN

RECEIVED BYISTORED IN $2009 / 123$

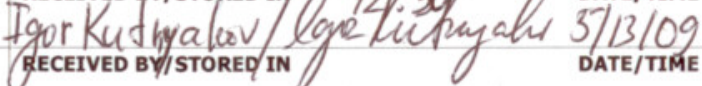

RECEIVED BY/STORED IN DATE/TIME

RECEIVED BY/STORED IN

DATE/TIM

RECEIVED BY/STORED IN

DATE/TIME

\begin{tabular}{|c|c|}
\hline $\begin{array}{c}\text { LABORATORY } \\
\text { SECTION }\end{array}$ & RECEIVED BY \\
\hline $\begin{array}{c}\text { FINAL SAMPLE } \\
\text { DISPOSITION }\end{array}$ & DISPOSAL METHO \\
\hline
\end{tabular}

TITLE

DISPOSED BY

\begin{tabular}{l} 
F09-012-047 \\
\hline PRICE CODE $\quad 8 \mathrm{~N}$ \\
AIR QUALITY \\
METHOD OF SHIPMENT \\
GOVERNMENT VEHICLE
\end{tabular}

PAGE 1 OF 1

PROJECT COORDINATOR

WIDRIG, DL

SAF NO.

COA

$300194 \mathrm{ES} 10$

GOVERNMENT VEHICLE

BILL OF LADING/AIR BILL NO.

N/A

DATA

Days
TOUN

45 Days / 45

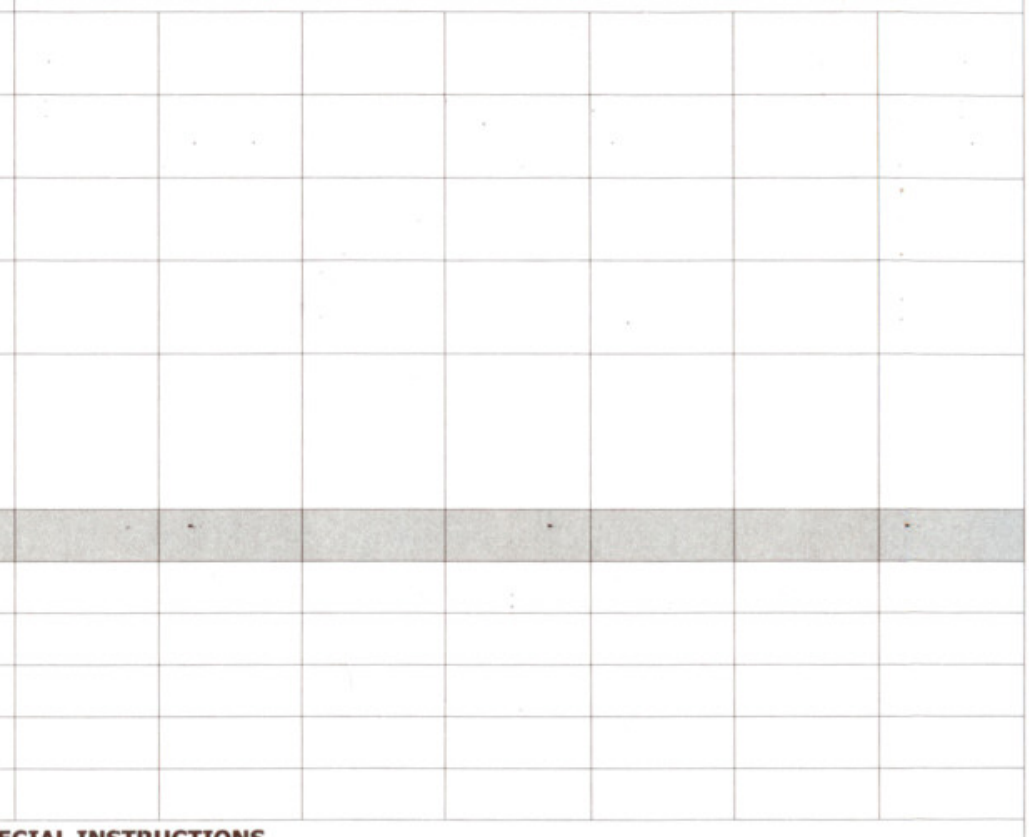

\section{SPECIAL INSTRUCTIONS}

applies to this SAF.

(1)TOC - ASTME1915A \{Total organic carbon\} GAMMA_GS \{Cesium-137, Cobalt-60, Europium-152, Europium-154, Europium-155\}

DISPOSI 
CH2MHill Plateau Remediation Company

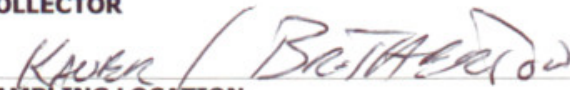
SAMPLING LOCATION

C7021; EW3-030

ICE CHEST NO.

\section{SHIPPED TO}

Environmental Sciences Laboratory

\begin{tabular}{|l|l}
\hline MATRIX* & POSSIBLE SAMPLE HAZARDS/ REMARKS \\
\hline
\end{tabular} $A=$ Air

$\mathrm{DL}=\mathrm{Drum}$

Liquids

$\mathrm{DS}=$ Drum

Solids

$\mathrm{L}=$ Liquid

$\mathrm{O}=$ Oil
$\mathrm{S}=$ Soil

$\mathrm{SE}=$ Sediment

$T=$ Tissue

$\mathrm{T}=\mathrm{T}$ issue
$\mathrm{V}=$ Vegitation

$\mathrm{W}=$ Water

WI $=$ Wipe

$\mathrm{X}=$ Other

Goods Regulations but a / IATA Dangerous Goods Regulations but are not releasable per

SPECIAL HANDLING AND/OR STORAGE RADIOACTIVE TIE TO: BIYNRO COLLECTOR

Contains Radioactive Material at concentrations that may or may not be regulated for DOE Order $5400.5(1990 / 1993)$

\section{CHAIN OF CUSTODY/SAMPLE ANALYSIS REQUEST}

COMPANY CONTAC

WIDRIG, DL

TELEPHONE NO.

376-2858

PROJECT DESIGNATION

200-ZP-1 Remedial Action Wells Sampling and Analysis - Geochemical Mode

FIELD LOGBOOK NO.

ACTUAL SAMPLE DEPTH

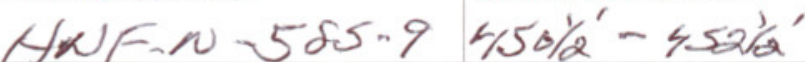
OFFSITE PROPERTY NO.

N/A

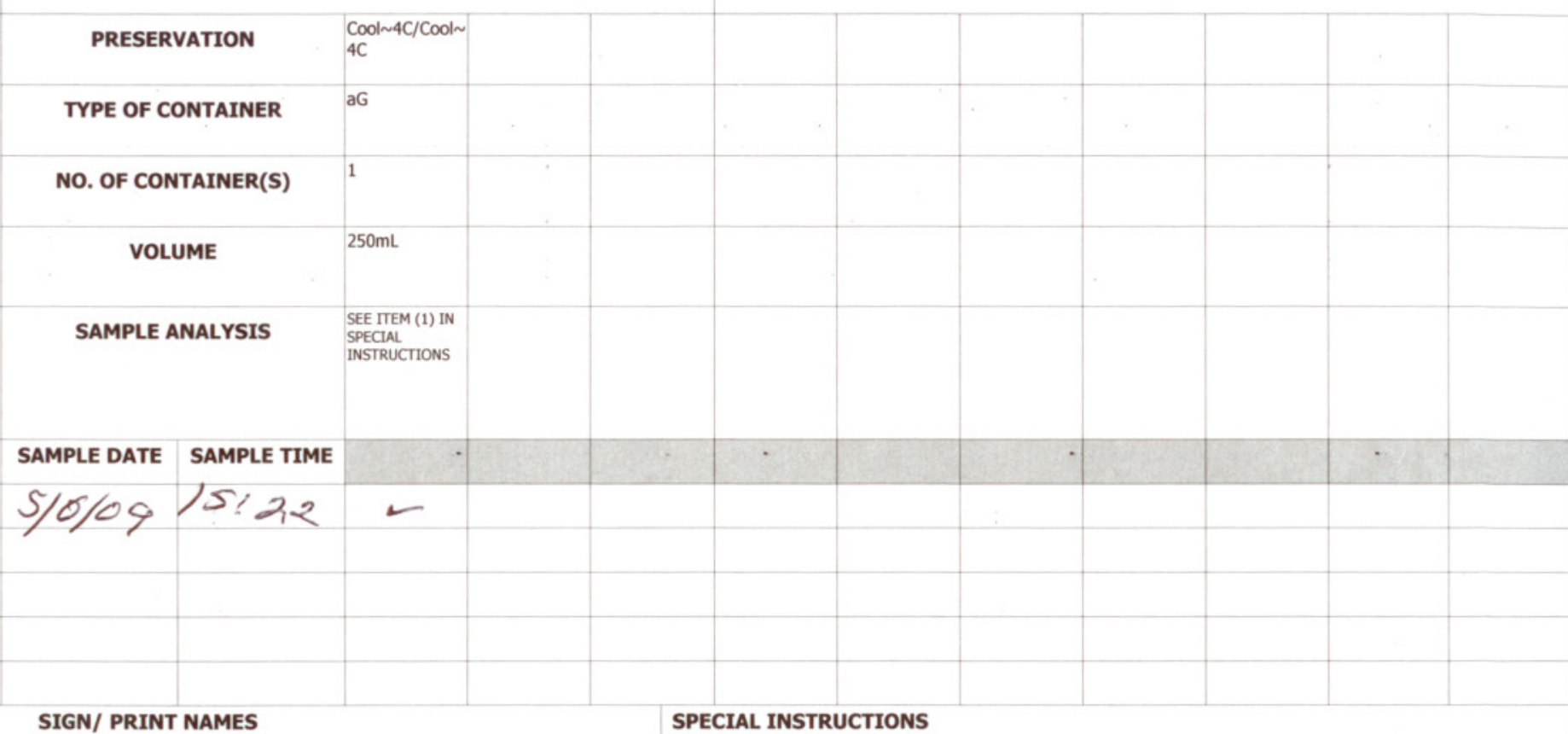

\section{SIGN/ PRINT NAMES}

\section{CHAIN OF POSSESSION}

RELINQUISHED BY/REMOVED FROM DATE/TIME REINOUISHED BY/REMOVED FROM DATE/TIME He yi3 $334-2 Z$ RELINQOISHED BY/REMOVED EBOM/DATE/TIME RCP altar Q MAY 1332009

RELINOUISHED BY/REMOVED FROM DATE/TIME

RELINQUISHED BY/REMOVED FROM DATE/TIME

RELINOUISHED BY/REMOVED FROM DATE/TIME

RELINQUISHED BY/REMOVED FROM DATE/TIME
RECEIVED BY/STORED IN KECEIVED BY/STORED IN (1230 DATE/TIME

** The 200 Area S\&GRP Characterization and Monitoring Sampling and Analysis GKI applies to this SAF.

(1)TOC - ASTME1915A \{Total organic carbon\} GAMMA_GS \{Cesium-137, Cobalt-60, Europium-152, Europium-154, Europium-155\} RECEIVED BY/STORED IN
F09-012-048

PAGE 1 OF 1

PRICE CODE

DATA OUND

WIDRIG, DL

SAF NO.

COA

AIR QUALITY

Days

GOVERNMENT VEHICLE

OF LADING/AIR BILL NO.

BILL O
METHOD OF SHIPMENT

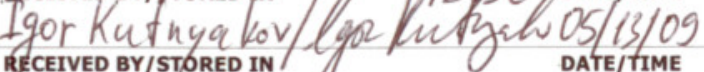

RECEIVED BY/STORED IN DATE/TIME

RECEIVED BY/STORED IN

DATE/TIME

RECEIVED BY/STORED IN

DATE/TIME

RECEIVED BY/STORED IN

DATE/TIME

\begin{tabular}{|c|c|c|c|}
\hline $\begin{array}{l}\text { LABORATORY } \\
\text { SECTION }\end{array}$ & RECEIVED BY & TITLE & DATE/TIME \\
\hline $\begin{array}{l}\text { FINAL SAMPLE } \\
\text { DISPOSITION }\end{array}$ & DISPOSAL METHOD & DISPOSED BY & DATE/TIME \\
\hline
\end{tabular}




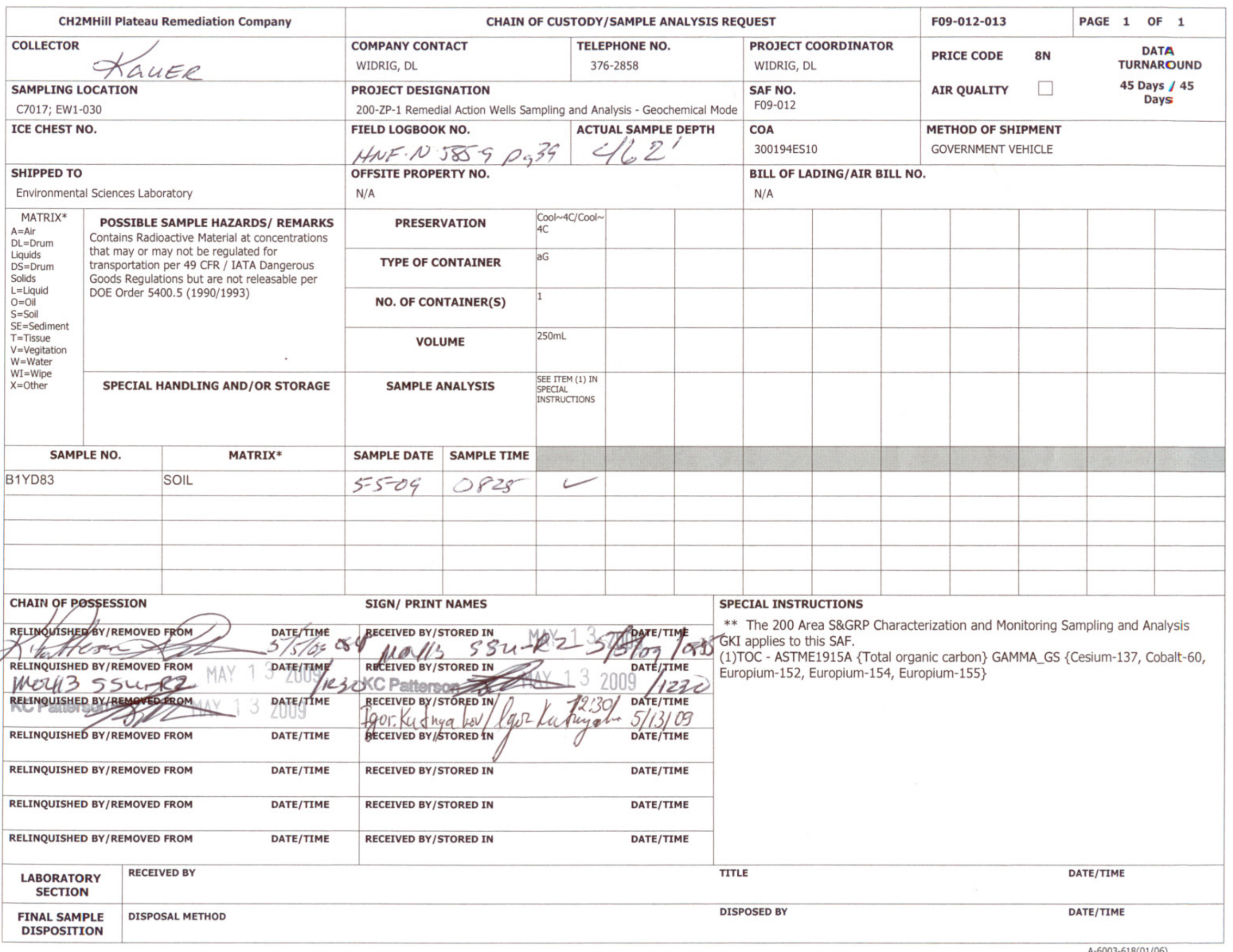




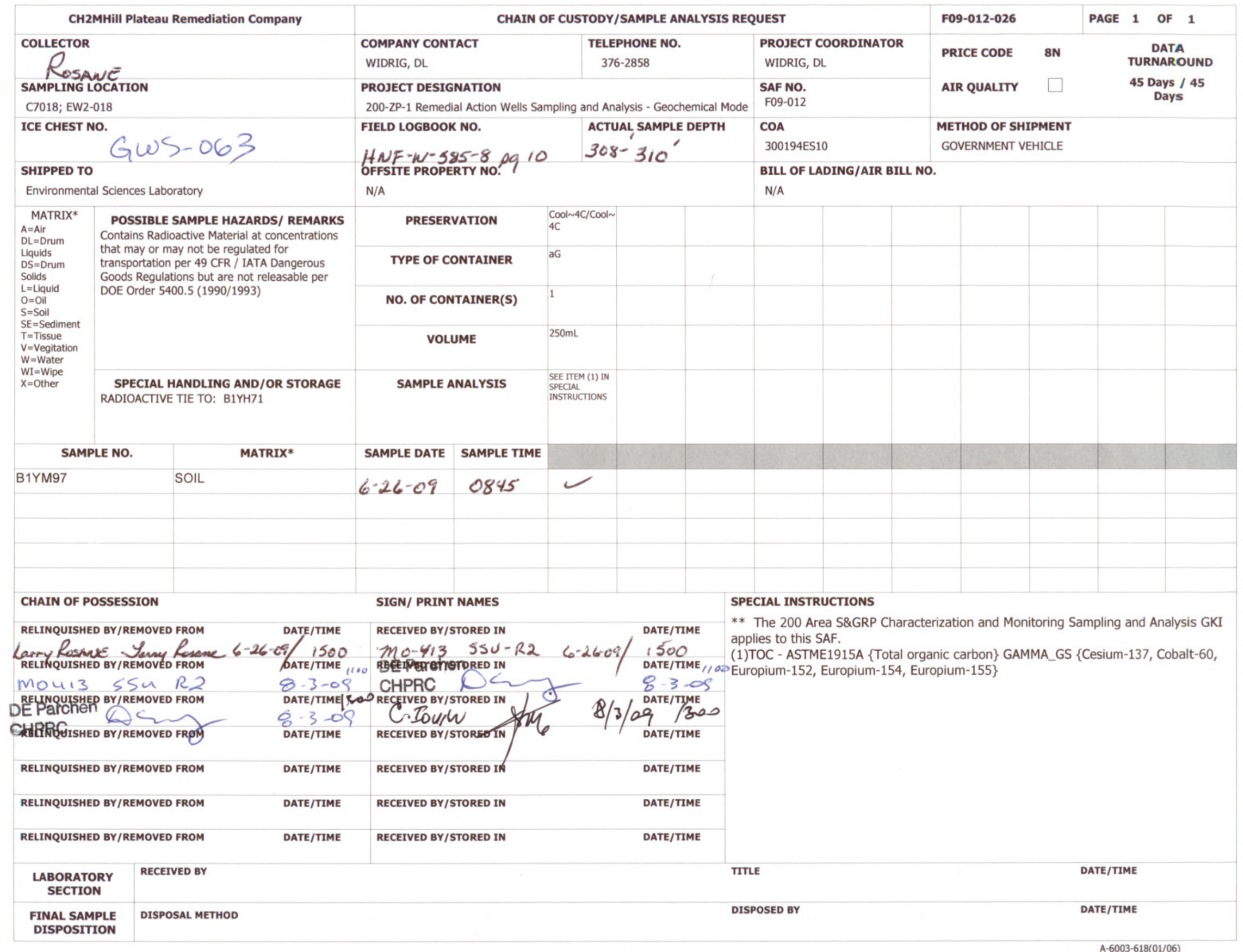

